\title{
Optimization Of Design And Manufacturing Process Of Fusion Filament Fabrication (FFF) 3D Printing
}

Jaeyoon Kim

Follow this and additional works at: https://researchrepository.wvu.edu/etd

\section{Recommended Citation}

Kim, Jaeyoon, "Optimization Of Design And Manufacturing Process Of Fusion Filament Fabrication (FFF) 3D Printing" (2018). Graduate Theses, Dissertations, and Problem Reports. 7197.

https://researchrepository.wvu.edu/etd/7197

This Dissertation is protected by copyright and/or related rights. It has been brought to you by the The Research Repository @ WVU with permission from the rights-holder(s). You are free to use this Dissertation in any way that is permitted by the copyright and related rights legislation that applies to your use. For other uses you must obtain permission from the rights-holder(s) directly, unless additional rights are indicated by a Creative Commons license in the record and/ or on the work itself. This Dissertation has been accepted for inclusion in WVU Graduate Theses, Dissertations, and Problem Reports collection by an authorized administrator of The Research Repository @ WVU.

For more information, please contact researchrepository@mail.wvu.edu. 


\title{
OPTIMIZATION OF DESIGN AND MANUFACTURING PROCESS OF FUSION FILAMENT FABRICATION (FFF) 3D PRINTING
}

\author{
Jaeyoon Kim \\ Dissertation submitted to the \\ College of Engineering and Mineral Resources at West Virginia University, \\ in partial fulfillment of the requirements for the degree of \\ Doctor of Philosophy \\ In \\ Mechanical Engineering
}

\author{
Bruce S. Kang, Ph. D., Chair \\ Jacky C. Prucz, Ph. D. \\ Ever J. Barbero, Ph. D. \\ Edward M. Sabolsky, Ph. D. \\ Hota V. Gangarao, Ph. D. \\ Department of Mechanical and Aerospace Engineering \\ West Virginia University \\ Morgantown, West Virginia, 2018
}

Keywords: Fused filament fabrication, 3D Printing, Tool-path, Finite element analysis, Composite Copyright 2018 Jaeyoon Kim 


\section{ABSTRACT \\ OPTIMIZATION OF DESIGN AND MANUFACTURING PROCESS OF FUSION FILAMENT FABRICATION (FFF) 3D PRINTING}

Jaeyoon Kim

Fused Filament Fabrication (FFF) is one of the most common Additive Manufacturing (AM) technologies for thermoplastic materials. Generally, AM enables to fabricate parts with more complex geometry. Structural optimization including topology and shape optimization has become more powerful to be used for the design of AM parts. Moreover, with the material advancement such as development of carbon fiber reinforced polymer (CFRP) filament for FFF, AM parts with improved strength and functionality can be realized. However, due to the anisotropic mechanical properties of AM parts induced by manufacturing process and intrinsic material characteristics, design methodology for AM engineering parts remains an active research area. In this research, a systematic optimization of design process of FFF 3D printing methodology is proposed for CFRP. Starting with structural optimization that reduces volume and finds the best geometry under the prescribed loading and boundary conditions. Standard coupon specimen tests including tensile, bending, and creep tests are carried out to obtain mechanical properties of CFRP. Finite element analyses (FEA) are conducted to find principal directions of the AM part and computed principal directions are utilized as fiber orientations. Then, the connecting lines of principal directions are used to develop a customized tool-path in FFF 3D printing to extrude fibers aligned with principle directions. Since current available infill-patterns in 3D printing cannot precisely draw customized lines, a specific tool-path algorithm has been developed to distribute fibers with the desired orientations. To predict/assess mechanical behavior of the AM part, 3D printing process was simulated followed by FEA to obtain the anisotropic mechanical behavior induced by the customized tool-path. To demonstrate the design/manufacturing methodology, lattice structure, stress concentration plate and spur gears of a ball milling machine were selected as case studies and carbon fiber reinforced nylon filament was chosen as the AM materials. Relevant tests were numerically and physically conducted to assess their performances. 


\section{ACKNOWLEDGEMENTS}

I would like to express my sincere appreciation to Dr. Bruce Kang for providing me the opportunity to extend my education. Dr. Kang taught me the way of critical thinking and abilities to learn new technologies and solve problems. He allowed freedom in research but had me stay on the right direction. I also want to extend appreciation to my committee members for their valuable contribution to my dissertation work: Dr. Jacky Prucz, Dr. Ever Barbero, Dr. Edward Sabolsky, and Dr. Hota Gangarao.

In addition to the important role Dr. Kang played in my training, many others were crucial for my growth as a researcher. All lab members at WVU that have helped me in different ways and are in need of recognition are Markova Mcpherson, Eric Chia, and Sizhe Huang. Finally, I express my deepest gratitude to my family who inspires me with endless love.

Jaeyoon Kim

June, 2018 


\section{TABLE OF CONTENTS}

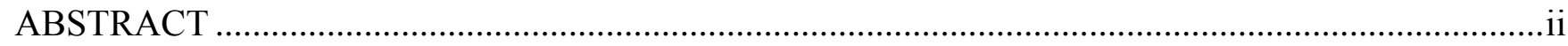

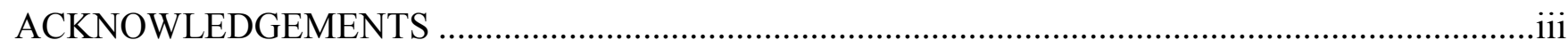

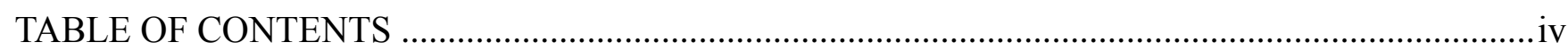

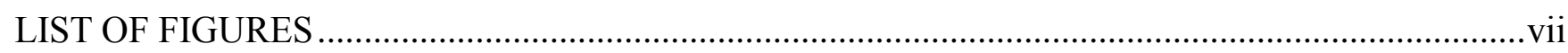

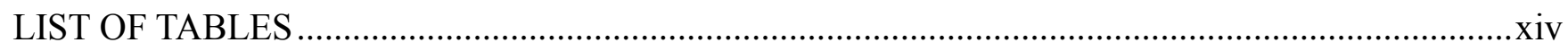

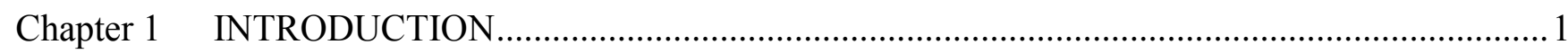

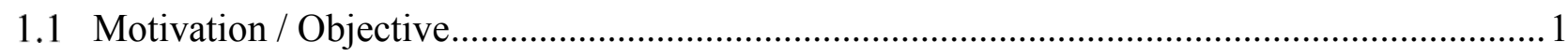

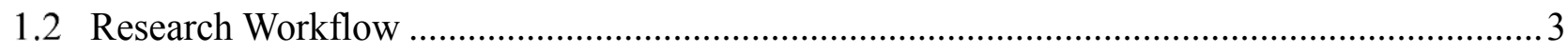

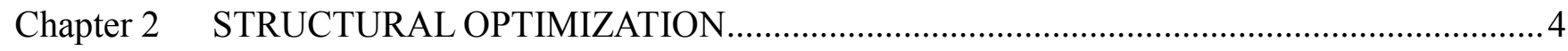

2.1 Topology Optimization....................................................................................................

2.1.1 Solid Isotropic Material Penalization (SIMP) ……......................................................

2.1.2 Bidirectional Evolutionary Structural Optimization (BESO) ........................................6

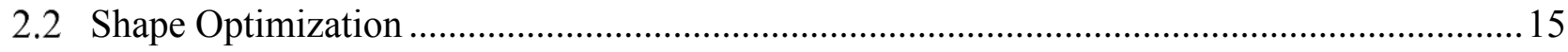

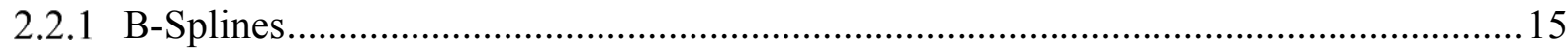

2.2.1 B-Spline Surface Mesh .......................................................................................... 16

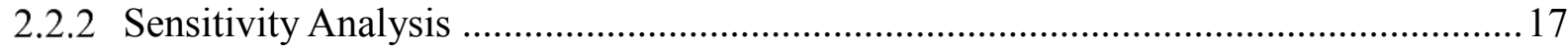

2.3 Design of Fiber Reinforcement ……………….................................................................. 19

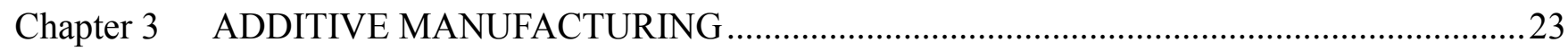

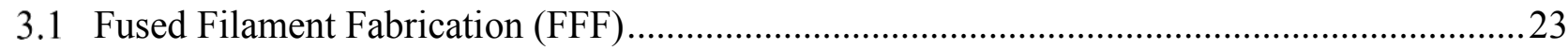

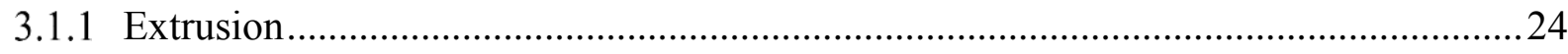

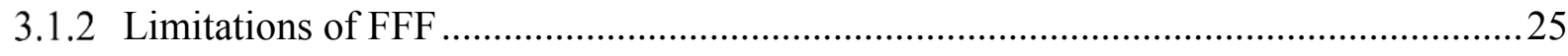

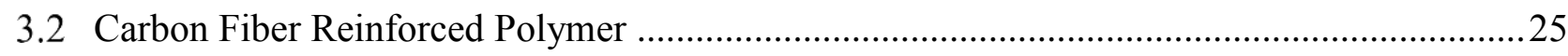

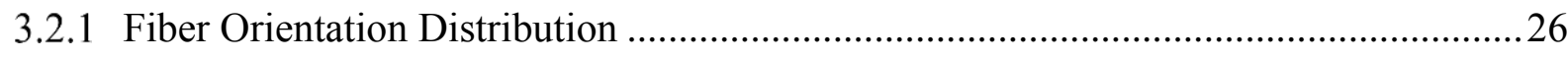

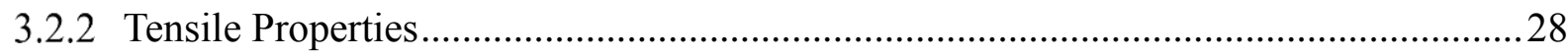




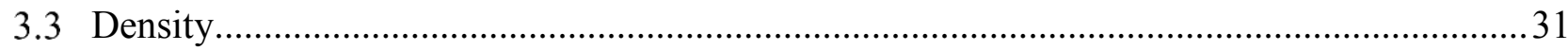

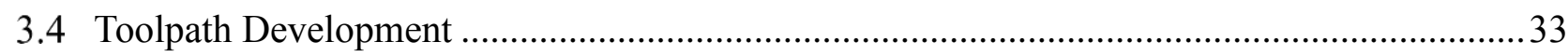

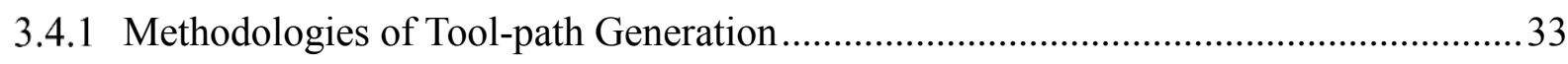

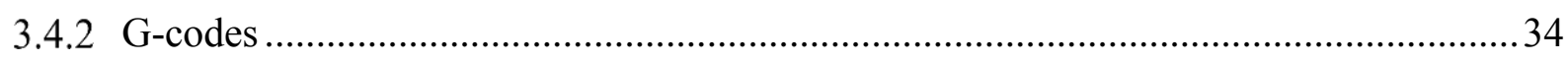

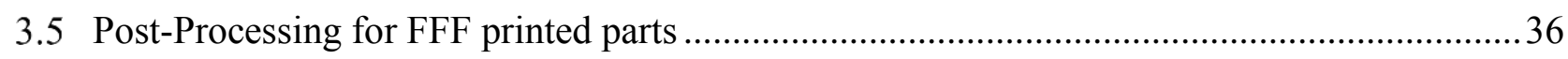

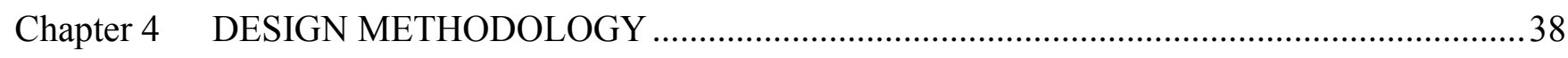

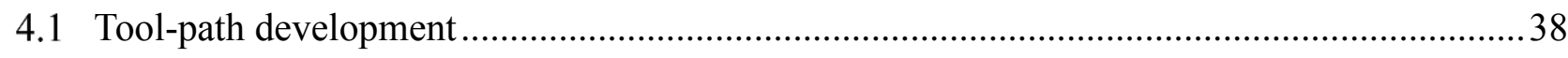

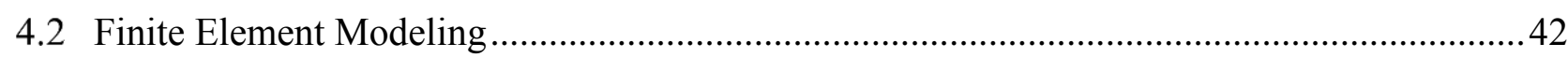

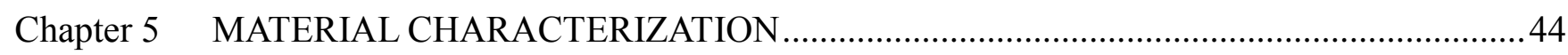

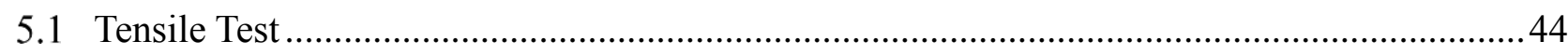

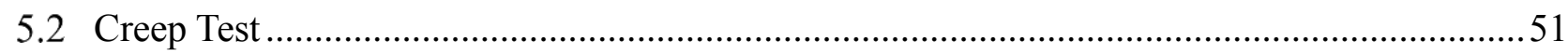

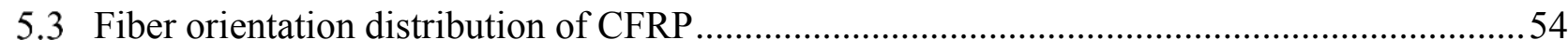

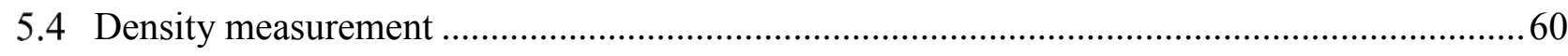

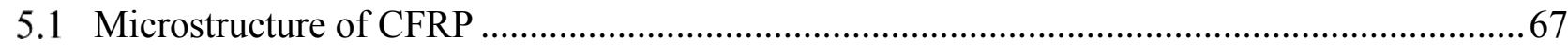

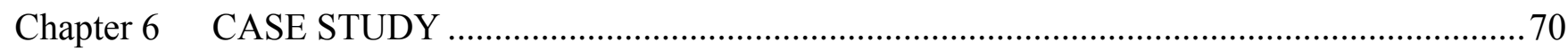

6.1 Case Study I - Lattice Structure …………………............................................................. 70

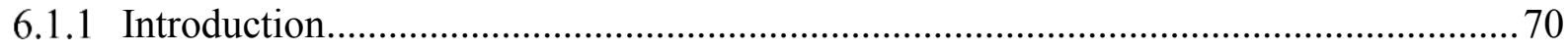

6.1.2 Structural Optimization ....................................................................................... 70

6.1.3 Determination of Fiber Orientations .......................................................................... 72

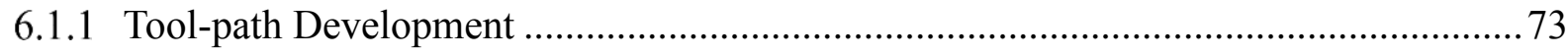

6.1.2 FE modeling and test simulation …………………………................................... 75

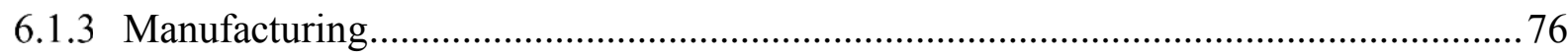

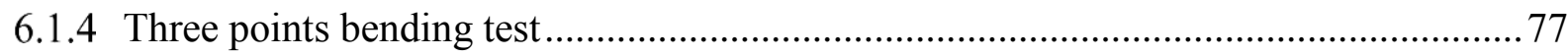

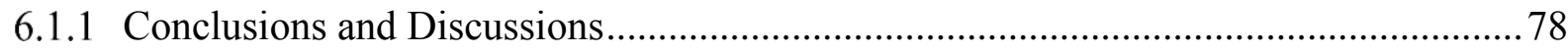

6.1 Case Study II. - Stress concentration plate ……………......................................................

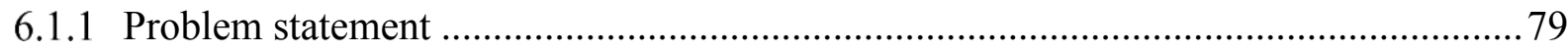




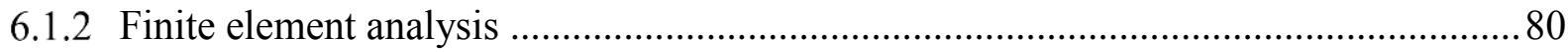

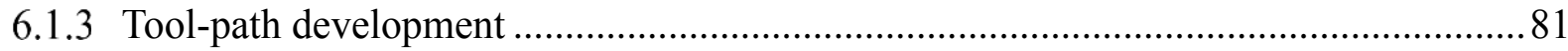

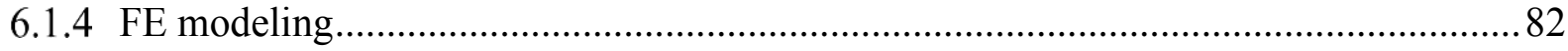

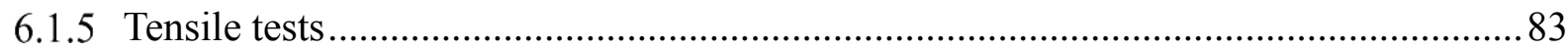

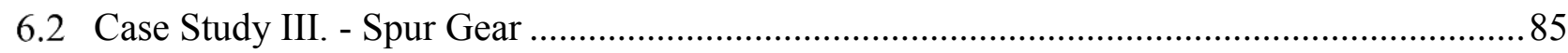

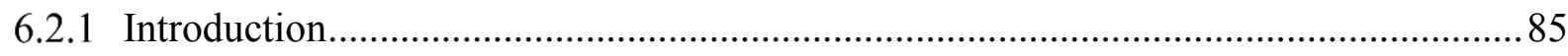

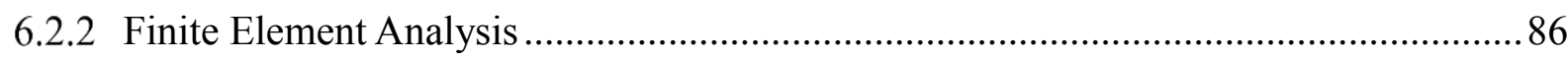

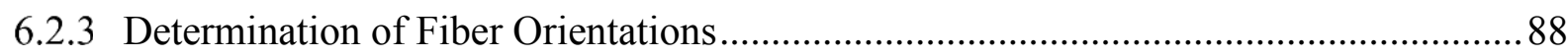

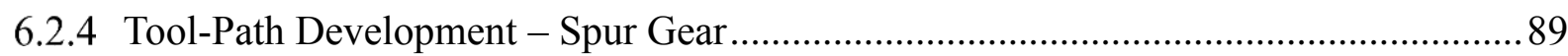

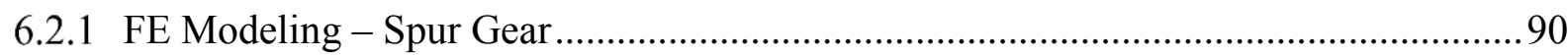

6.2.2 Compression Test - Spur Gear ………………..................................................... 91

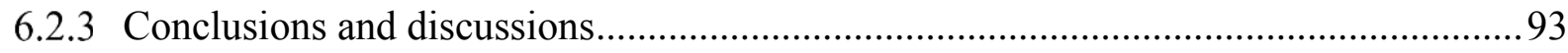

6.2.4 Part Application for Ball-Milling Machine....................................................................94

6.2.5 Post-Processing - Spur Gear ................................................................................. 96

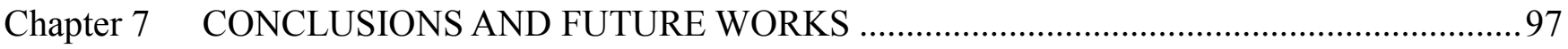

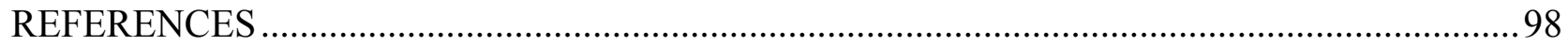

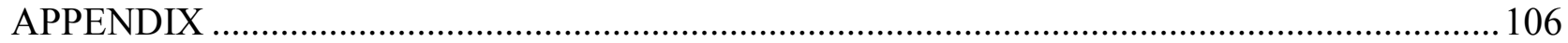




\section{LIST OF FIGURES}

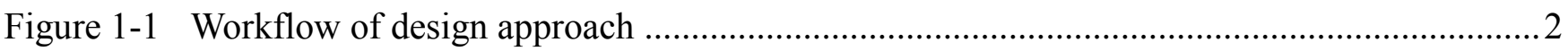

Figure 2-1 Normalized stiffness vs density relations in topology optimization methods [104] .............5

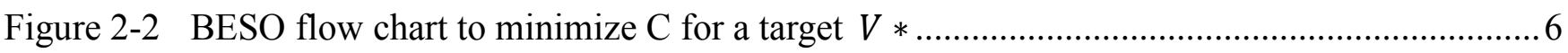

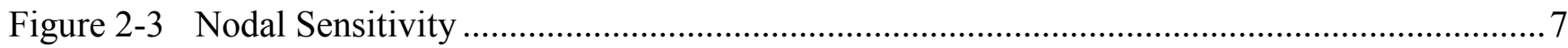

Figure 2-4 Checkerboard effect for two-phase field and the associated filtered fields [11] ................... 8

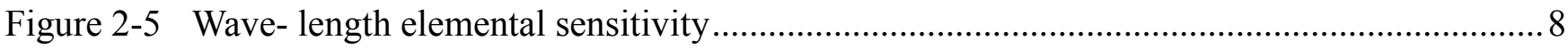

Figure 2-6 Element removal \& addition ....................................................................................

Figure 2-7 An example of BESO method (a) inital, (b) iteration 300, (c) iteration 45, (d) final topology. [16]

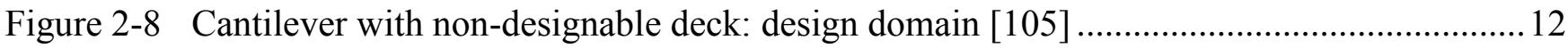

Figure 2-9 Final topology of the cantilever with displacement constraint $(\mathrm{V}=49.8 \%$, dmax $=$

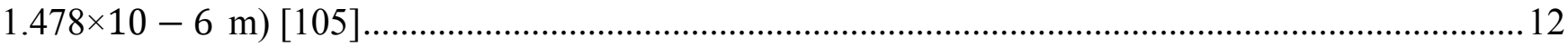

Figure 2-10 Final topology from stiffness optimal design $(\mathrm{V}=49.8 \%, \mathrm{dmax}=1.834 \times 10-6 \mathrm{~m})$ [105]

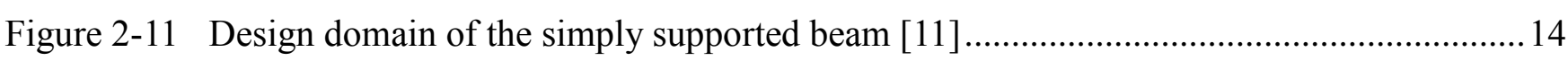

Figure 2-12 The first two eigenmodes of the optimal design: (a) the first eigenmode (b) the second

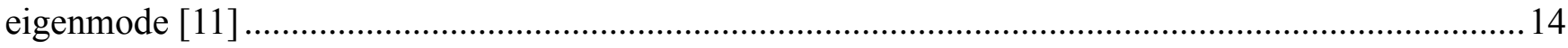

Figure 2-13 Effect of knot spacing. Solid line: uniform knot vector. Dotted line: non-uniform knot ... 16

Figure 2-14 B-spline surface mesh for a case $(n=3, p=2, m=2, q=1, U=\{0,0,0,1 / 2,1,1,1\}, V=\{0,0$,

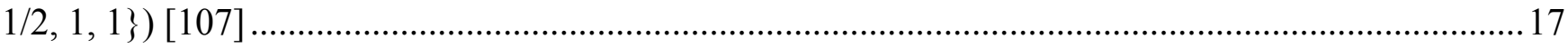

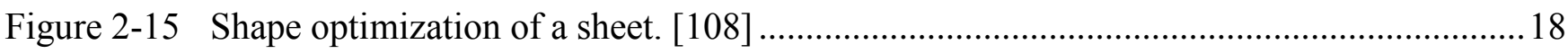

Figure 2-16 Initial mesh and optimized shape (10 iterations) of a cantilever plane sheet [108] .........19

Figure 2-17 Illustration of the concept of orientation optimization in a finite element analysis [109] .20

Figure 2-18 Test example objective function: (a) the candidate materials at 12 angles, i ; and (b) the white triangles mark possible combinations of candidate materials [109] .............................................. 21

Figure 2-19 Optimal fiber angle distribution using 768 elements and a single candidate material at $\left[90, \pm 75, \pm 60, \pm 45, \pm 30, \pm 15,0^{\circ}\right][109]$

Figure 2-20 Optimal fiber angle distribution using 768 elements in 48 patches of $4 \times 4$ elements [109]

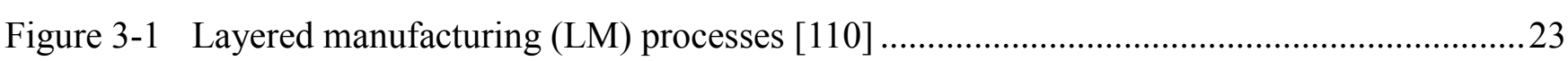

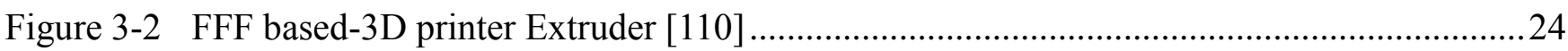

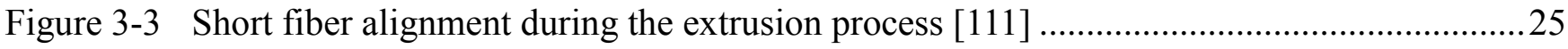

Figure 3-4 Sketch of a dog-bone sample showing orientation directions [70] ……..............................28

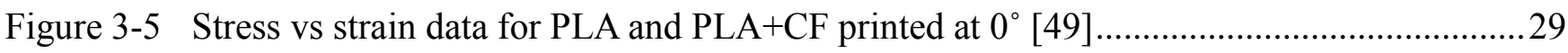

Figure 3-6 Stress vs strain data for PLA and PLA+CF printed at 90 [49] .........................................30 
Figure 3-7 Effect of fiber content and preparation process on (a) tensile strength, and (b) modulus, of

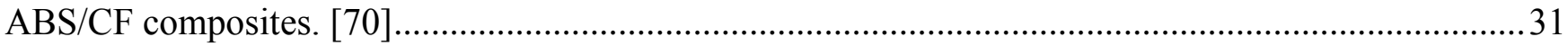

Figure 3-8 Cross-section of beads with lower (a) and higher (b) SR[112] .........................................32

Figure 3-11 Comparison of different tool-path generation strategies; (a) Direction parallel path , (b)

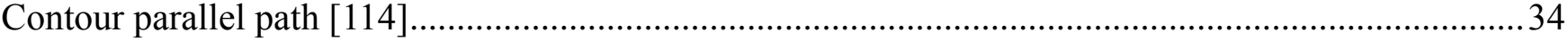

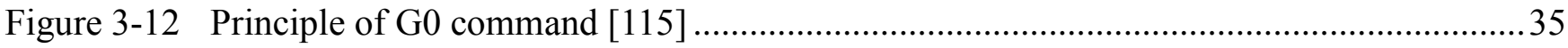

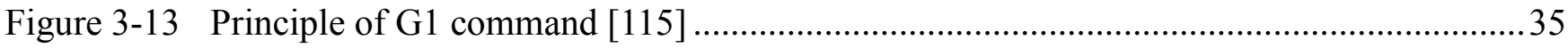

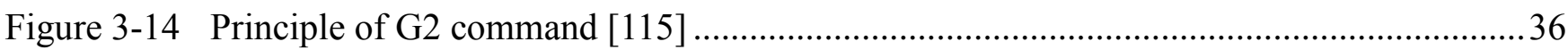

Figure 3-15 Annealing process. A) initial cold state, B) Heating: high stress areas dissipate, C)

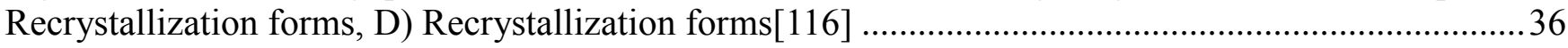

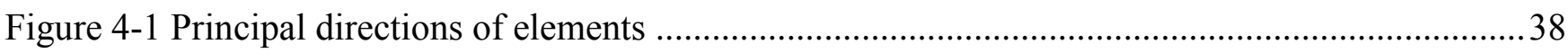

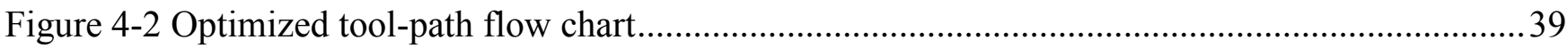

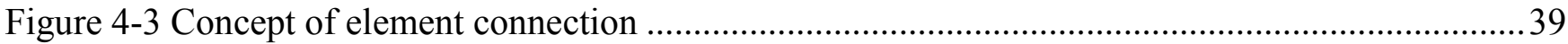

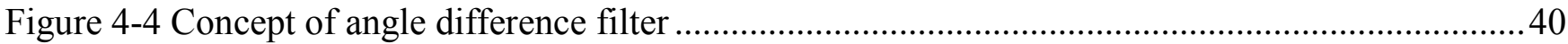

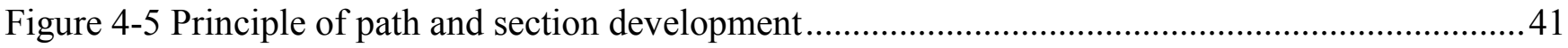

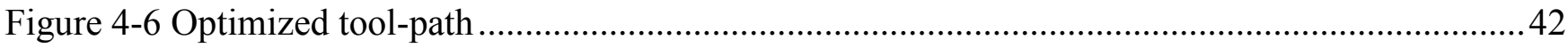

Figure 4-7 Comparison of modeling between different number of total elements; $100 * 100$ of left, $50 * 50$

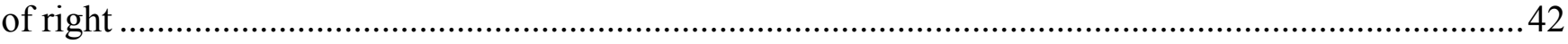

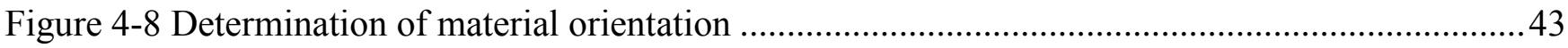

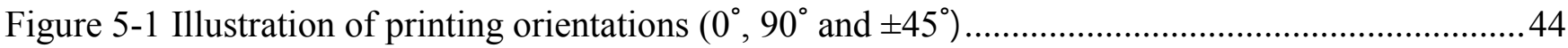

Figure 5-2 Employed specimens (a): tensile (“dog bone”, lower) and shear (rectangular, upper) and

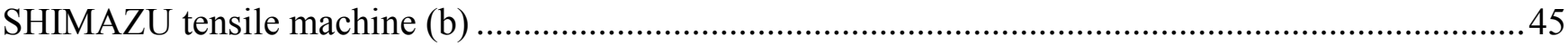

Figure 5-3 raw data (a) and curve fitting (b) of strain stress curve of CFRP-nylon printed at $0^{\circ}$ direction

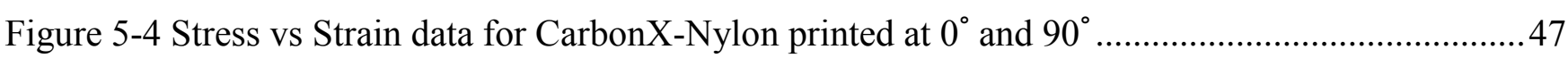

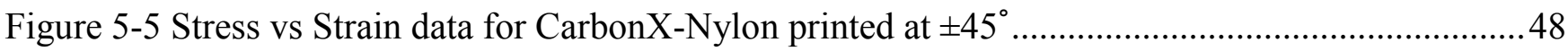

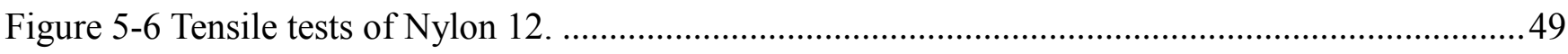

Figure 5-7 Tensile test result of Nylon 12 samples printed at various angles ....................................50

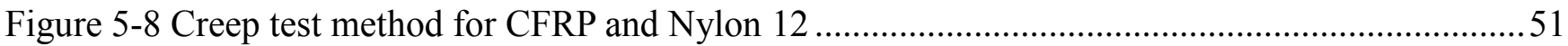

Figure 5-9 Creep test results of CFRP and Nylon 12with different fiber orientations.........................55

Figure 5-10 Comparison of creep response for CFRP and Nylon 12 ...........................................53

Figure 5-11 Sample micrographs of a polished specimen obtained from optical microscope...............54

Figure 5-12 (a) Elliptical cross section of cylindrical fiber showing definition of axes' endpoints and inplane angle $\Phi,(b)$ Cross sections of a fiber inclined 0 from the 3 -axis. [70] .....................................55

Figure 5-13 fiber capture converting pixels into black and white...............................................55

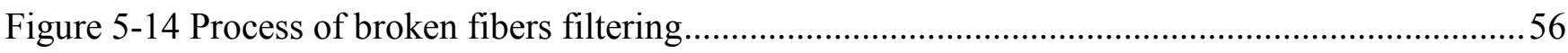


Figure 5-15 Definition of Eulerian angles showing components of unit vector p.[70]......................56

Figure 5-16 Fiber orientation distribution of $a 11$ component .....................................................58

Figure 5-17 Fiber orientation distribution of $a 12$ component ......................................................59

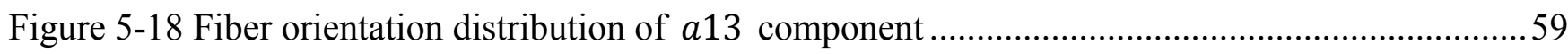

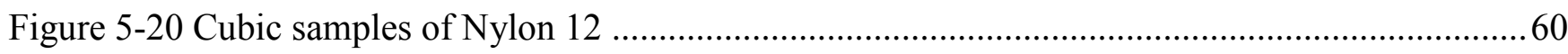

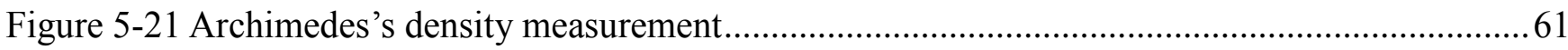

Figure 5-22 Relative densities of samples printed by various nozzle size and layer height .................62

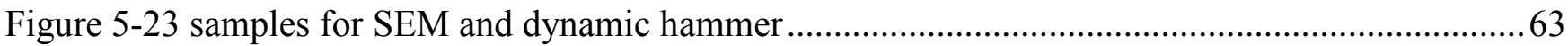

Figure 5-24 SEM image of a sample printed by $0.4 \mathrm{~mm}$ nozzle with $0.1 \mathrm{~mm}$ layer height....................64

Figure 5-25 SEM image of a sample printed by $0.6 \mathrm{~mm}$ nozzle with $0.15 \mathrm{~mm}$ layer height.................65

Figure 5-26 SEM image of a sample printed by $0.8 \mathrm{~mm}$ nozzle with $0.15 \mathrm{~mm}$ layer height.................65

Figure 5-27 Tensile test results of samples printed by various nozzle sizes ....................................66

Figure 5-28 Tensile failure of a specimen printed by $0.8 \mathrm{~mm}$ nozzle .............................................67

Figure 5-29 SEM image of CFRP specimen printed at $0^{\circ}$ direction $(150 \mathrm{x})$...................................6 68

Figure 5-30 SEM image of CFRP specimen printed at $0^{\circ}$ direction $(250 \mathrm{x})$....................................68

Figure 5-31 SEM image of CFRP specimen printed at $45^{\circ}$ direction ............................................6 69

Figure 5-32 SEM image of CFRP specimen printed at $90^{\circ}$ direction............................................69

Figure 6-1 Quad-copter with an optimized frame [117] ................................................... 70

Figure 6-2 An example of topology optimization for a simply-sported beam subjected to three points

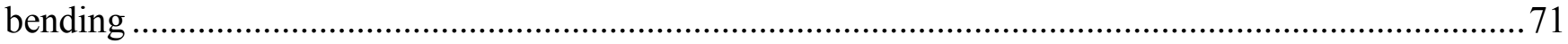

Figure 6-3 Evolution history of volume fraction and global stiffness ...........................................71

Figure 6-4 Evolution of the topology: (a) iteration 5, (b) iteration 11 and (c) final topology................72

Figure 6-5 An example of optimal fiber angle distribution [109] .............................................. 72

Figure 6-6 Section division for customized tool-path.............................................................. 73

Figure 6-7 Illustration showing a direction-parallel tool-path segments [118] ..............................73

Figure 6-8 Material deposition scheme of FFF 3D printing for a section.......................................74

Figure 6-9 Customized tool-path for the optimized structure .................................................74

Figure 6-10 FE modeling of the optimized beam..........................................................................75

Figure 6-11 Comparison of maximum failure load between uniformly aligned and optimally distributed

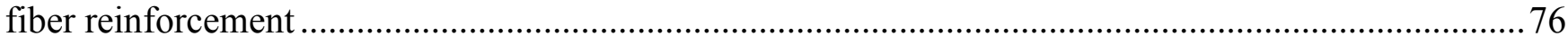

Figure 6-12 Optimized beam with carbon fiber reinforcement by FFF 3D printing ........................76

Figure 6-13 Upgraded Ultimaker 2+ in Dr. Kang's lab. ........................................................... 77

Figure 6-14 An example of nozzle damage and ruby nozzle.................................................... 77

Figure 6-15 Three points bending test for the optimized beam.....................................................77

Figure 6-16 Comparison of load vs displacement curves of beams with different printing pattern ........78 
Figure 6-17 Comparison of results between FEA and Experimental tests ......................................79

Figure 6-18 (a) Stress flow and (b) a FFF built CFRP sample of stress concentration plate ..................79

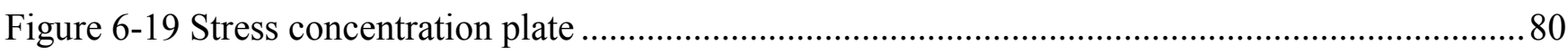

Figure 6-20 (a) stress field and (b) principal directions of stress concentration plate ..........................80

Figure 6-21 (a) Section division for tool-path and (b) complete optimized tool-path of stress concentration

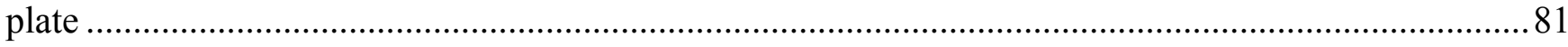

Figure 6-22 Stress distribution of the plate printed by the optimized tool-path...................................82

Figure 6-23 shear strain distribution of samples printed by (a) $0^{\circ}$ uniform; (b) customized tool-path....82

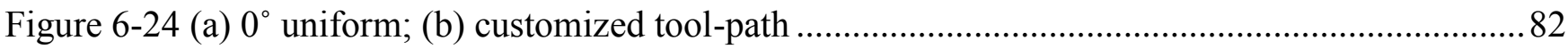

Figure 6-25 (a) tensile test and (b) test result of stress concentration plate ......................................83

Figure 6-26 comparison of stiffness response between FEA simulations and physical experiments ......84

Figure 6-27 Spur gear damages of the ball-milling machine .................................................... 85

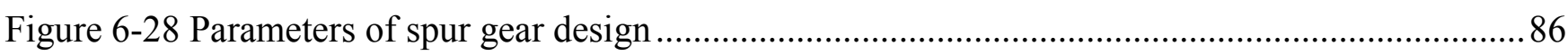

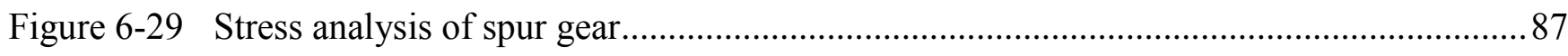

Figure 6-30 Maximum stress index for isotropic gear pairs ................................................. 88

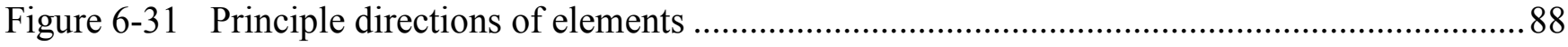

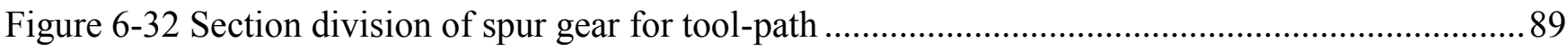

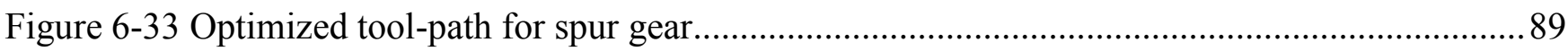

Figure 6-34 FE modeling (a) and compression test simulation (b) .............................................. 90

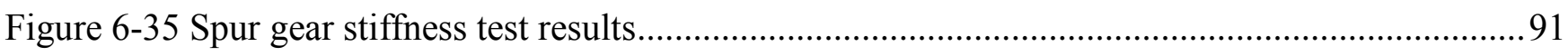

Figure 6-36 Spur gear stiffness test for CFRP (a) and Nylon 12 (b) ............................................ 91

Figure 6-37 Load vs Displacement curves of CFRP spur printed by various tool-path........................92

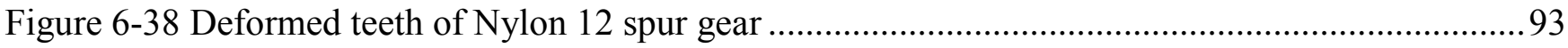

Figure 6-39 Comparison of results between FEA simulation and experimental tests..........................94

Figure 6-40 Gear set of ball milling machine, (a) damaged original gears (b) replacement of CFRP gears

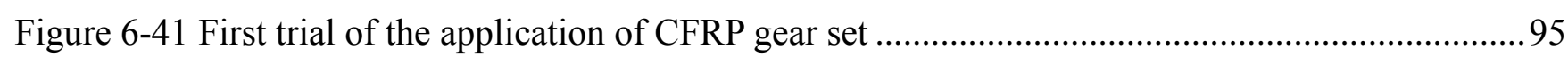

Figure 6-42 Stiffness test results of spur gears after post-processing ..........................................96

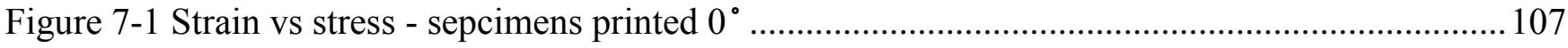

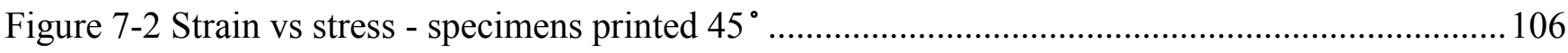

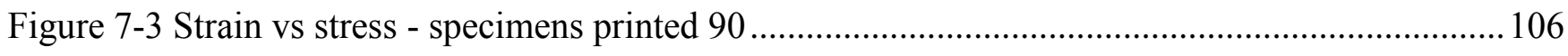

Figure 7-4 SEM image of a sample printed by $0.4 \mathrm{~mm}$ nozzle ..................................................... 113

Figure 7-5 SEM image of a sample printed by $0.4 \mathrm{~mm}$ nozzle ....................................................... 113

Figure 7-6 SEM image of a sample printed by $0.6 \mathrm{~mm}$ nozzle ..................................................... 114

Figure 7-7 SEM image of a sample printed by $0.6 \mathrm{~mm}$ nozzle ................................................... 114 
Figure 7-8 SEM image of a sample printed by $0.8 \mathrm{~mm}$ nozzle 115

Figure 7-9 SEM image of a sample printed by $0.8 \mathrm{~mm}$ nozzle

Figure 7-10 Fiber orientation computation process of a microscope image (cross-section of $0^{\circ}$ direction, 20x).

Figure 7-11 Fiber orientation computation process of a microscope image (cross-section of $0^{\circ}$ direction, 20x).

Figure 7-12 Fiber orientation computation process of a microscope image (cross-section of $0^{\circ}$ direction, 20x).

Figure 7-13 Fiber orientation computation process of a microscope image (cross-section of $0^{\circ}$ direction, 20x). 120

Figure 7-14 Fiber orientation computation process of a microscope image (cross-section of $0^{\circ}$ direction, 20x). 121

Figure 7-15 Fiber orientation computation process of a microscope image (cross-section of $0^{\circ}$ direction, 20x). 121

Figure 7-16 Fiber orientation computation process of a microscope image (cross-section of $0^{\circ}$ direction, 20x). 122

Figure 7-17 Fiber orientation computation process of a microscope image (cross-section of $0^{\circ}$ direction, 20x). 122

Figure 7-18 Fiber orientation computation process of a microscope image (cross-section of $0^{\circ}$ direction, 20x). 123

Figure 7-19 Fiber orientation computation process of a microscope image (cross-section of $0^{\circ}$ direction, 20x).

Figure 7-20 Fiber orientation computation process of a microscope image (cross-section of $0^{\circ}$ direction, 20x). 124

Figure 7-21 Fiber orientation computation process of a microscope image (cross-section of $0^{\circ}$ direction, 20x). 124

Figure 7-22 Fiber orientation computation process of a microscope image (cross-section of $0^{\circ}$ direction, 20x). 125

Figure 7-23 Fiber orientation computation process of a microscope image (cross-section of $0^{\circ}$ direction, 20x).

Figure 7-24 Fiber orientation computation process of a microscope image (cross-section of $0^{\circ}$ direction, 20x). 126

Figure 7-25 Fiber orientation computation process of a microscope image (cross-section of $0^{\circ}$ direction, 20x). 126

Figure 7-26 Fiber orientation computation process of a microscope image (cross-section of $0^{\circ}$ direction, 20x).

Figure 7-27 Fiber orientation computation process of a microscope image (cross-section of $0^{\circ}$ direction, 20x).

Figure 7-28 Fiber orientation computation process of a microscope image (cross-section of $0^{\circ}$ direction, 20x). 128 
Figure 7-29 Fiber orientation computation process of a microscope image (cross-section of $0^{\circ}$ direction, 20x).

Figure 7-30 Fiber orientation computation process of a microscope image (cross-section of $90^{\circ}$ direction, 20x).

Figure 7-31 Fiber orientation computation process of a microscope image (cross-section of $90^{\circ}$ direction, 20x). 129

Figure 7-32 Fiber orientation computation process of a microscope image (cross-section of $90^{\circ}$ direction, 20x). 130

Figure 7-33 Fiber orientation computation process of a microscope image (cross-section of $90^{\circ}$ direction, 20x). 130

Figure 7-34 Fiber orientation computation process of a microscope image (cross-section of $90^{\circ}$ direction, 20x).

Figure 7-35 Fiber orientation computation process of a microscope image (cross-section of $90^{\circ}$ direction, 20x).

Figure 7-36 Fiber orientation computation process of a microscope image (cross-section of $90^{\circ}$ direction, 20x).

Figure 7-37 Fiber orientation computation process of a microscope image (cross-section of $90^{\circ}$ direction, 20x).

Figure 7-38 Fiber orientation computation process of a microscope image (cross-section of $90^{\circ}$ direction, 20x).

Figure 7-39 Fiber orientation computation process of a microscope image (cross-section of $90^{\circ}$ direction, 20x).

Figure 7-40 Fiber orientation computation process of a microscope image (cross-section of $90^{\circ}$ direction, 20x).

Figure 7-41 Fiber orientation computation process of a microscope image (cross-section of $90^{\circ}$ direction, 20x).

Figure 7-42 Fiber orientation computation process of a microscope image (cross-section of $90^{\circ}$ direction, 20x).

Figure 7-43 Fiber orientation computation process of a microscope image (cross-section of $90^{\circ}$ direction, 20x).

Figure 7-44 Fiber orientation computation process of a microscope image (cross-section of $90^{\circ}$ direction, 20x). 136

Figure 7-45 Fiber orientation computation process of a microscope image (cross-section of $90^{\circ}$ direction, 20x). 136

Figure 7-46 Fiber orientation computation process of a microscope image (cross-section of $90^{\circ}$ direction, 20x). 137

Figure 7-47 Fiber orientation computation process of a microscope image (cross-section of $90^{\circ}$ direction, 20x)..... 137

Figure 7-48 Fiber orientation computation process of a microscope image (cross-section of $90^{\circ}$ direction, 20x). 138 
Figure 7-49 Fiber orientation computation process of a microscope image (cross-section of $90^{\circ}$ direction,

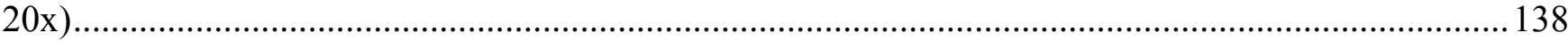

Figure 7-50 Three points bending test results of the beam printed at Optimized, $0^{\circ}, 45^{\circ}$, and $90^{\circ}$, respectively 139

Figure 7-51 Load vs displacement for the gear printed at the optimized tool-path ................................ 140

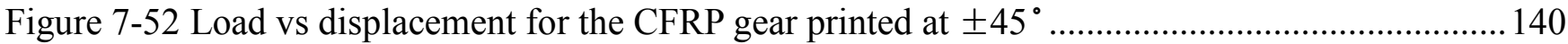

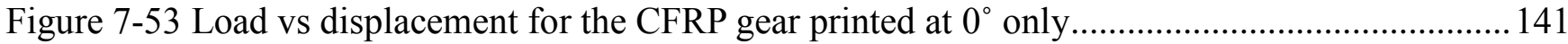

Figure 7-54 Load vs displacement for the Nylon 12 gear printed at optimized tool-path ....................... 141

Figure 7-55 Load vs displacement for the CFRP gear after annealing under $140{ }^{\circ} \mathrm{C}$............................. 142

Figure 7-56 7-57 Load vs displacement for the CFRP gear after annealing under $140^{\circ} \mathrm{C}$....................... 142

Figure 7-58 Tensile stress vs strain of samples printed by $0.4 \mathrm{~mm}$ nozzle ............................................. 143

Figure 7-59 Tensile stress vs strain of samples printed by $0.6 \mathrm{~mm}$ nozzle ............................................. 143

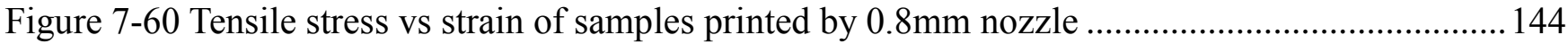




\section{LIST OF TABLES}

Table 3-1 Components of the second-order orientation tensor of ABS/CF composites [70] ...............227

Table 3-2 Previous tensile tests of composites produced by FFF and injection molding (IM) ..............28

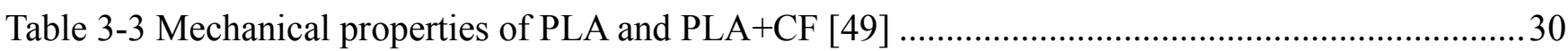

Table 5-1 Comparison of mechanical properties between isotropic pure Nylon 12 and CarbonX-Nylon .

Table 5-2 Second-order orientation tensor values for CFRP fibers.................................................5 57

Table 5-3 Experimental data of Archimedies's density test ...........................................................62

Table 6-1 Tensile test results of stress concentration plate printed by various tool-path ......................83

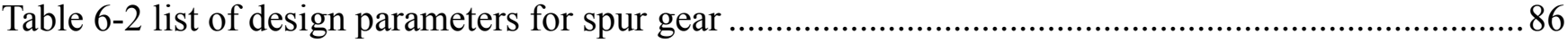

Table 6-3 test results of stiffness response of CFRP and Nylon 12 ..............................................92 


\section{Chapter 1 INTRODUCTION}

\subsection{Motivation / Objective}

Additive manufacturing (AM) technologies have been rapidly advancing and widening its applicability to complex geometries and range of material choice. Since complex geometry can be easily realized by AM, structural optimization (SO) technique has become a powerful tool to generate optimal design for AM parts. Researchers have achieved unprecedented success in integrating AM technology with conventional SO techniques to design and manufacture engineering parts with reduced weight and optimal performance. However, due to the inherent nature of AM process, there has been a limitation for SO to be fully applied to the design of AM parts. Layer by layer manufacturing induces anisotropic mechanical properties. SO technique is not able to control this anisotropic mechanical property by itself when it designs AM parts. In this research, a novel methodology integrating SO, fiber placement theory, and tool-path development theory to design and manufacture AM parts is proposed. Fused filament fabrication (FFF) 3D printing method was chosen as AM tech for research. FFF is one of the most widely used in AM technologies for thermoplastic material. Carbon fiber reinforced polymer (CFRP) was chosen as material with intrinsic anisotropy. For CFRP, extensive research has been carried out to investigate the anisotropic mechanical properties of CFRP including ABS, PLA, and nylon. Generally the anisotropic

structural property of FFF parts is highly dependent on the building direction. The study of the building direction has been highlighting only the selection of its orientation. For FFF 3D printing, slicer programs provide several limited infill patterns to choose a building direction. Once a pattern is selected, it is not allowed to edit its tool-path. This is because tool-path for FFF was originally developed to control the movement of CNC machine cutter. When the tool-path algorithm was developed for FFF, it was for printing process improvement, doesn't consider the structural strength enhancement of final products. The proposed design methodology starts with an FEA stress analysis. From on the output principal directions, customized tool-path was developed. Tensile tests and selective electron microscope (SEM) for CFRP-nylon was performed to investigate mechanical properties and fiber orientations. Structural performances of FFF parts built by the proposed tool-path method was verified by both computational and physical experiments. The framework for this methodology is shown in Figure 1-1. 
Primary contributions are as follows:

(1) A novel design methodology for FFF parts assisted by finite element analysis (FEA)

(2) A customized tool-path algorithm for FFF that maximize the effect of fiber reinforcement under the given loading and boundary conditions.

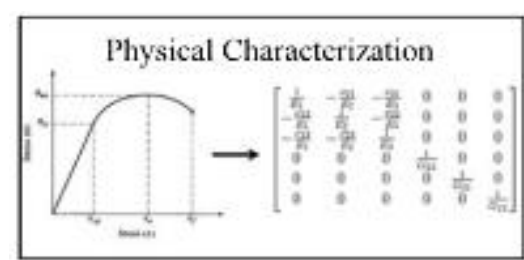

CAD Model \& Stress Analysis
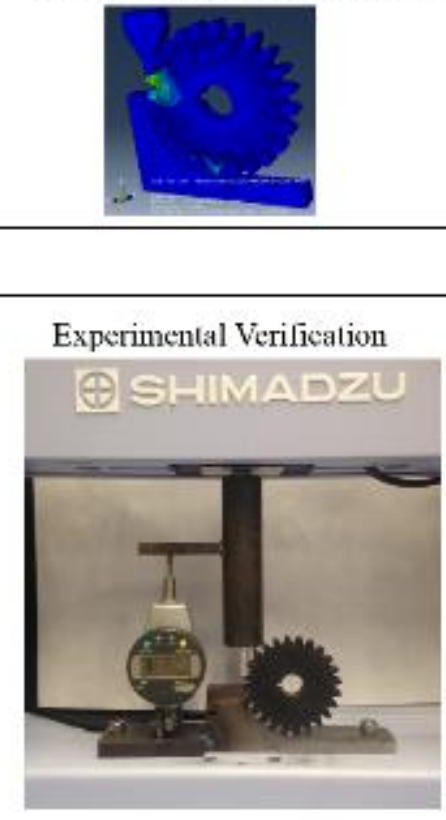

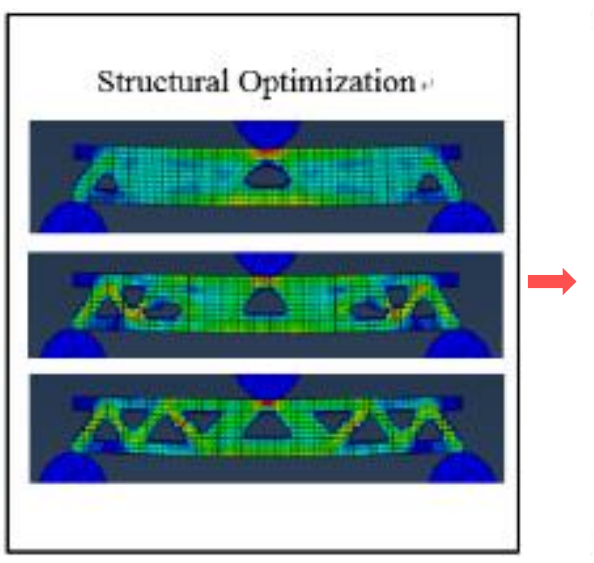

FEA Simulation of 3D Printing
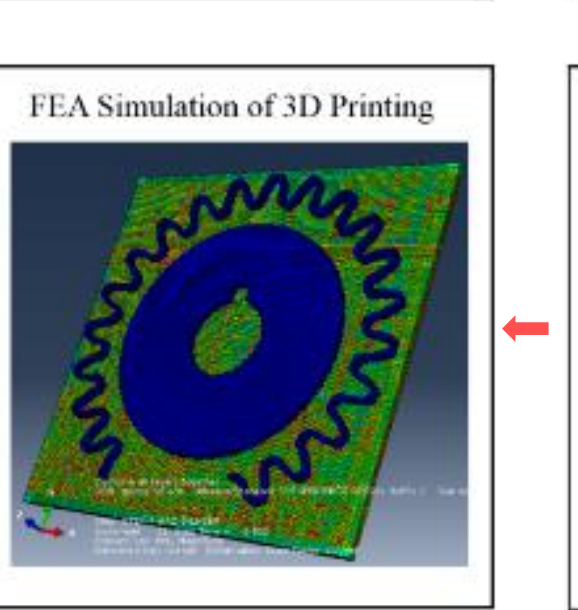

Determination of fiber orientation
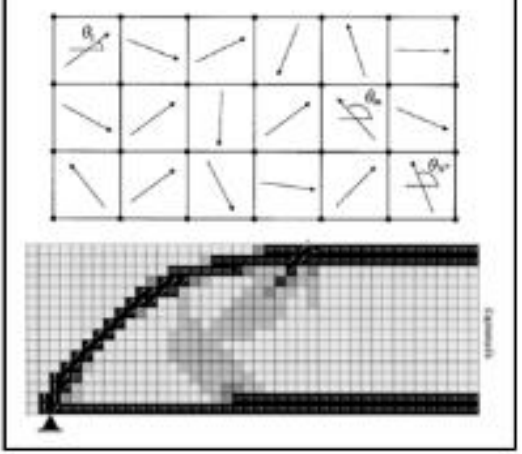

$\downarrow$

Tool-Path Development

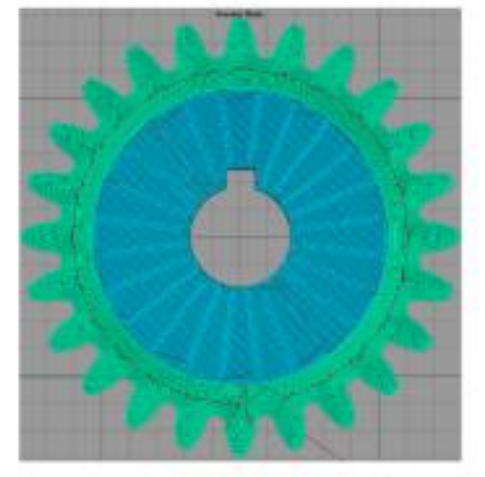

Figure 1-1 Workflow of design approach 


\subsection{Research Workflow}

Step 1: Conduct FEM analysis with isotropic material under given loading conditions. ABAQUS 6.14 is utilized.

Step 2: Perform topology and shape optimization with certain constraints (e.g volume, natural frequency or displacement). In this step, ABAQUS Topology Optimization Module is utilized.

Step 3: Compute elemental principle directions using the output from ABAQUS. Then, determine the optimal fiber orientations of individual elements.

Step 4: Divide printing sections with the identical fiber orientations. Next, develop customized toolpath for each section. Finally, generate g-codes. For step $3 \& 4$, program coding is established by MATLAB 2016 and Simplify3D 4.0.

Step 5: In ABAQUS assign anisotropic materials with optimal orientations to the divided sections from Step 3.

Step 6: Convert the g-codes from Step 4 into python in ABAQUS. Then, simulate FFF 3D printing process to evaluate the residual stress of final products induced during the manufacturing. GENOA 8.0 is utilized for g-codes conversion.

Step 7: Perform FEM analysis again with the updated and optimally designed structure to assess the improvement.

Step 8: Fabricate 3D printed products and perform scanning electron microscope (SEM) analysis to see fiber distribution and control voids.

Step 9: Verify the performance of the printed products. Replace the original gear parts of a planetary ball milling machine with newly printed parts, check its durability. Moreover, three points bending tests for optimized lattice structures are carried out to evaluate the effect of the optimal fiber mapping. 


\section{Chapter 2 STRUCTURAL OPTIMIZATION}

\subsection{Topology Optimization}

AM processes require the development of new design theories. Current design theories were developed prior to AM suited to traditional manufacturing. These theories constrain the complexity of solutions achieved. With traditional manufacturing, this can be advantageous as it can minimize manufacturing difficulties whereas this is not the case for AM. Topology optimization (TO), however, provides greater potential for AM, since it is capable of achieving solutions for complex geometries.

Topology optimization is one of the types of structural optimization that seeks the optimum layout of a design by determining the number of members (elements) required in the design [1]. Algorithms developed for TO include homogenization[1,2], solid isotropic microstructure with Penalization (SIMP)[3]-[5] and evolutionary structural optimization (ESO)[6 , 7], Stochastic algorithms used in the broader field of optimization have also been adopted for TO, including genetic algorithms [8, 9] and ant colony optimization[10]. From these theories, bi-directional evolutionary structural optimization (BESO) [11] which is an advanced version of ESO is applied to this research. In this chapter, the principle concept of SIMP theory is briefly discussed. Then, BESO and its applications are reviewed in detail.

\subsubsection{Solid Isotropic Material Penalization (SIMP)}

SIMP method was developed in the 1980s. It is called "material interpolation", "artificial material", "power law", or "density" method, but "SIMP" is now used fairly universally. The term "SIMP" stands for Solid Isotropic Microstructure (or Material) with Penalization for intermediate densities. The basic idea of this approach was proposed by Bendsoe [12], while the term "SIMP” was named later by Rozvany et al [3]. In SIMP approach, the design domain is discretized into small rectangular elements. Within each discretized element, material properties are assumed constant and isotropic and the design variable is the element density. The SIMP approach penalizes intermediate density for binary topology patterns using a penalization factor to assign lower stiffness values as shown Figure 2-1. 


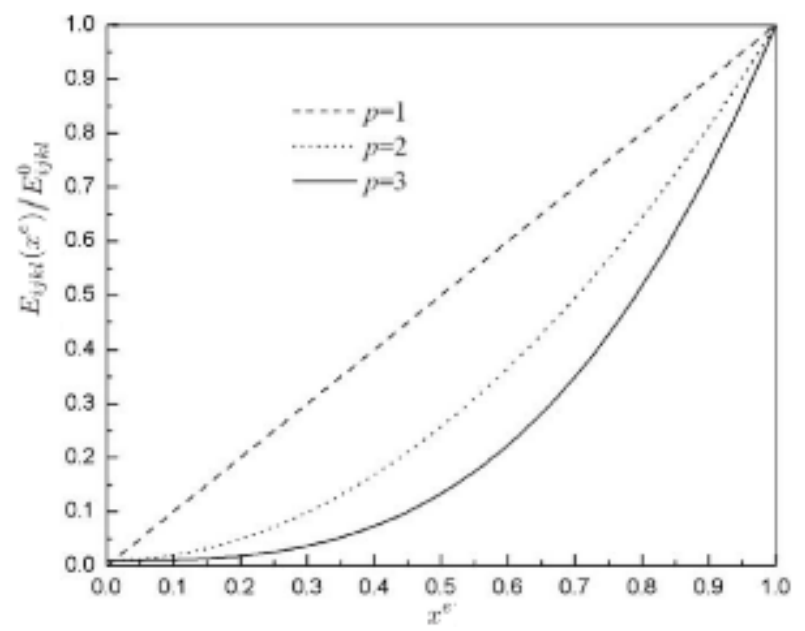

Figure 2-1 Normalized stiffness vs density relations in topology optimization methods [104] Considering structures built from one material and void, there's a basic assumption of relationship between stiffness tensor $\operatorname{Eijkl}\left(x^{e}\right)$ and relative density of the element $x^{e}$ :

$$
E_{i j k l}\left(x^{e}\right)=\left(x^{e}\right)^{p} E_{i j k l}^{0}, p>1
$$

where $\mathrm{E}_{i j k l}^{0}$ is the stiffness tensor of a solid element. The penalty factor $p$ is key feature of the SIMP algorithm. Based on the assumption of Eq 2-1, a topology optimization problem based on the SIMP approach where the objective is to minimize compliance can be written as

$$
\begin{gathered}
\min _{\mathbf{x}}: c(\mathbf{x})=\mathbf{U}^{T} \mathbf{K} \mathbf{U}=\sum_{e=1}^{N}\left(x^{e}\right)^{p} \mathbf{u}_{e}^{T} \mathbf{k}_{0} \mathbf{u}_{e} \\
\text { s.t.: }\left\{\begin{array}{c}
\mathbf{K U}=\mathbf{F} \\
\frac{V(\mathbf{x})}{V_{0}}=f \\
0<\mathbf{x}_{\min } \leq \mathbf{x} \leq 1
\end{array}\right.
\end{gathered}
$$

where $\mathrm{x}$ represents design variables. $\mathrm{U}$ and $\mathrm{F}$ are the global displacement and force vectors. respectively. $\mathrm{K}$ represents the global stiffness matrix, $u_{e}$ and $k_{0}$ represents the element displacement vector and the element stiffness matrix. $\mathrm{N}$ represents the number of elements. $\mathrm{V}(\mathrm{x})$ and $V_{0}$ is the material volume and design domain volume, respectively. And $\mathrm{f}$ is the prescribed volume fraction. Then, Sensitivity of objective function can be easily calculated as

$$
d c=\frac{\partial c}{\partial x^{e}}=-p\left(x^{e}\right)^{p-1} \mathbf{u}_{e}^{T} \mathbf{k}_{0} \mathbf{u}_{e}
$$




\subsubsection{Bidirectional Evolutionary Structural Optimization (BESO)}

One of the most recent, advanced and widely used TO approach for AM is bi-directional evolutionary structural optimization (BESO)[6][13][14][15][16][17]. This is a finite element (FE)-based TO method, where inefficient material is iteratively removed from a structure while efficient material is simultaneously added to the structure. BESO is introduced as an algorithm for minimizing the strain energy, C, of the aerospace part at two volume fraction constraints, V. Two optimization parameters are varied systematically. The general methodology is to develop a FE-based model of the system under load and then to seek the optimal design for a given set of conditions. BESO[16][17] requires a number of steps, involving both finite element analysis (FEA), filtering and optimization. Key steps are shown in the flowchart given in Figure 2-2

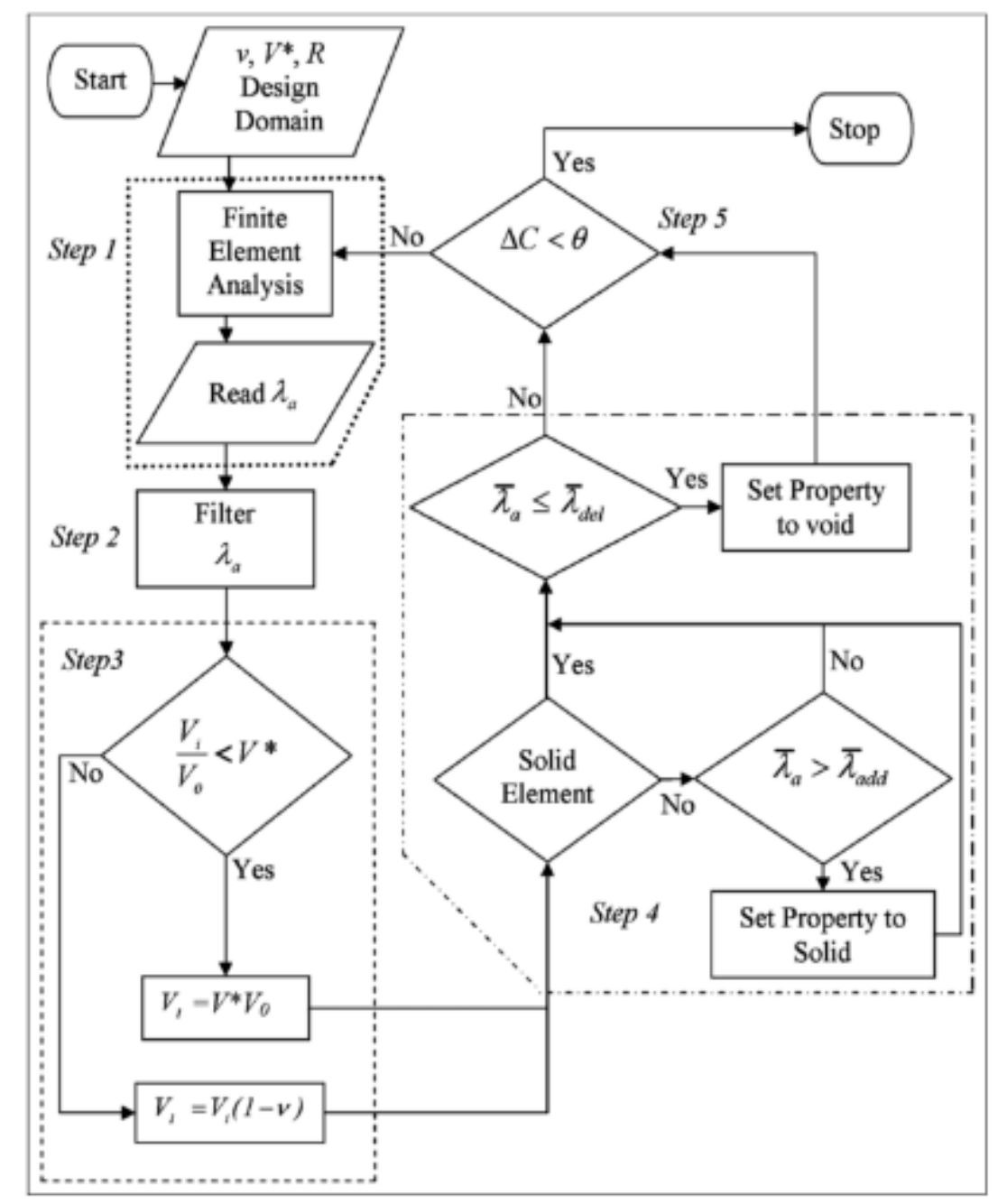

Figure 2-2 BESO flow chart to minimize $\mathrm{C}$ for a target $V^{*}$ 
For a load $F$, causing displacement $u$, the problem can be mathematically expressed as

$$
\begin{aligned}
& \min : C=\frac{1}{2} F^{t} u \\
& \text { subject to }: \frac{V_{i}}{V_{0}}=V^{*}, \quad V_{i}=\sum_{a=1}^{P} V_{a}
\end{aligned}
$$

where $F^{t}$ is the transpose of the force vector, $V_{0}$ is the initial volume of a design and $V_{i}$ is the volume of a design at iteration, $i$, computed by summing the volume, $V_{a}$ of each element, $a$, at this iteration. $P$ is the total number of elements in a mesh.

From Step 2 in the flowchart, the elemental sensitivities, $\lambda_{a}$ are equivalent to the elemental strain energies. These elemental strain energies are filtered in two stages. First, a volume weighting of the sensitivities of the elements connected to a node, $b$, is computed as shown Figure 2-3

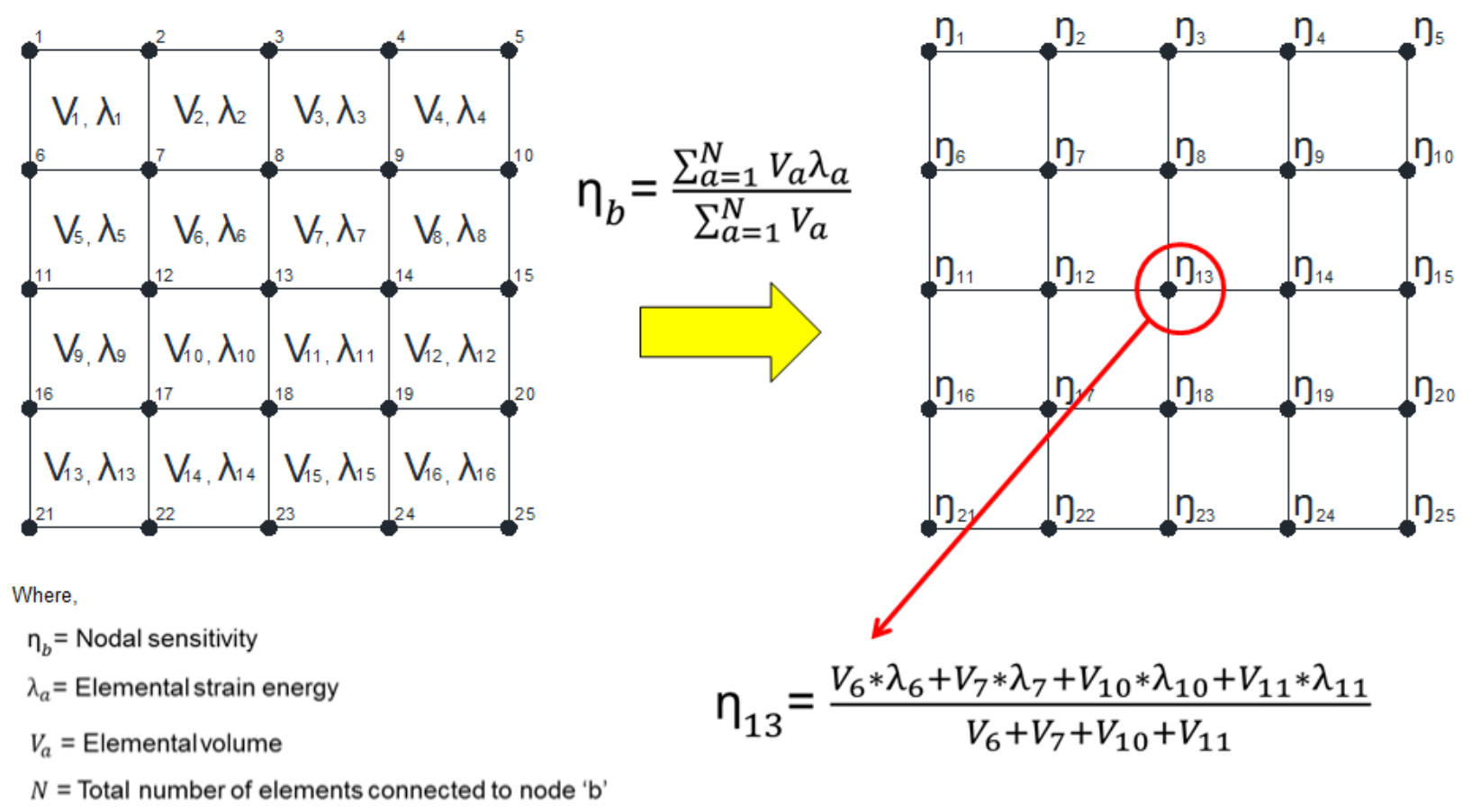

Figure 2-3 Nodal Sensitivity

Second, a longer wave-length elemental sensitivity, $\bar{\lambda}_{c}$, is calculated by finding nodes $d$ whose distance $r$ to the center of an element $c$ is less than or equal to the filter radius, R. Figure 2-5 illustrates the computation. 

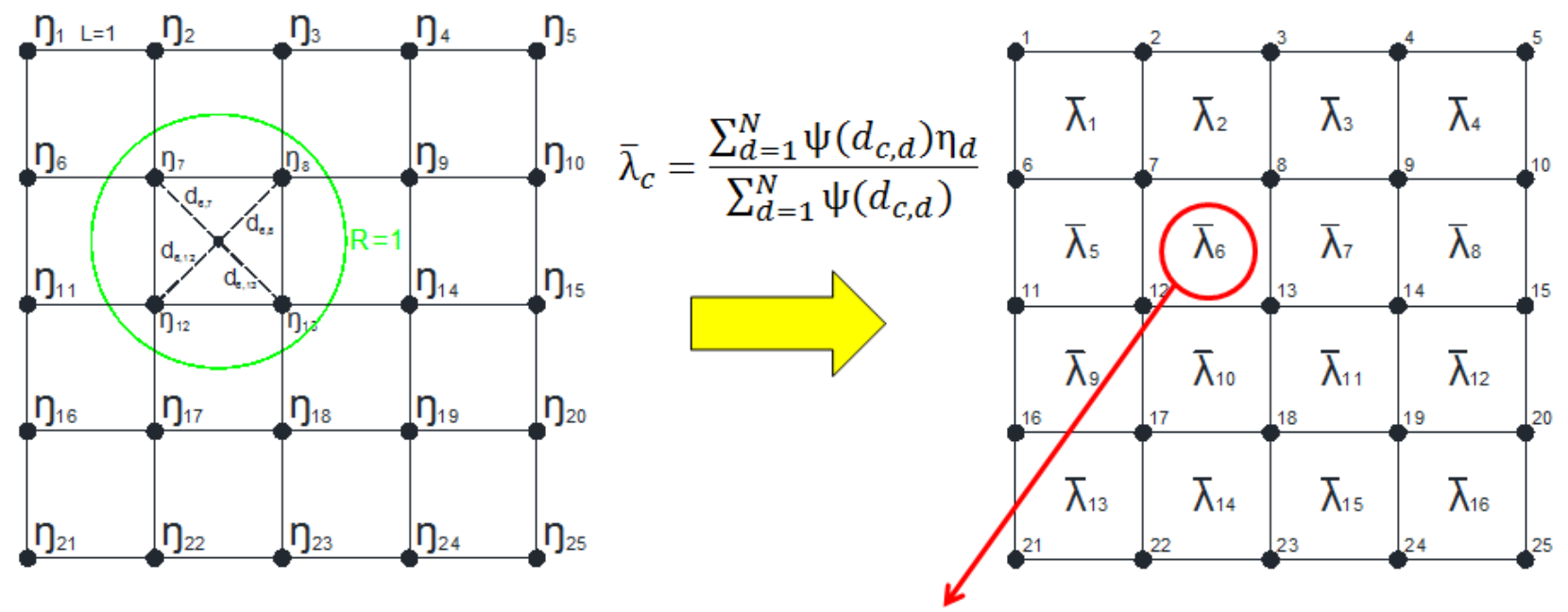

Where,

$\bar{\lambda}_{c}=$ Wave length elemental sensitivity

$\psi\left(d_{c, d}\right)=\mathrm{R}-d_{c, d} \quad \eta_{d}=$ Nodal sensitivity

$$
\bar{\lambda}_{c}=\frac{\left(R-d_{6,7}\right) * \eta_{7}+\left(R-d_{6,8}\right) * \eta_{8}+\left(R-d_{6,12}\right) * \eta_{12}+\left(R-d_{6,13}\right) * \eta_{13}}{\left(R-d_{6,7}\right)+\left(R-d_{6,8}\right)+\left(R-d_{6,12}\right)+\left(R-d_{6,13}\right)}
$$

$\mathrm{R}=$ User-defined scale factor

Then, $\quad \bar{\lambda}_{c}=\frac{\bar{\lambda}_{c}^{i}-\bar{\lambda}_{c}^{i-1}}{2} \quad \bar{\lambda}_{c}^{i}=$ current sensitivity at iteration ' $\mathrm{i}$ '

$d_{c, d}=$ Distance between node ' $\mathrm{d}$ ' and the center of element ' $\mathrm{c}$ '

$\bar{\lambda}_{c}^{i-1}=$ sensitivity at the previous iteration

$N=$ Total number of nodes in the sub-domain with radius ' $\mathrm{R}$ '

Figure 2-5 Wave- length elemental sensitivity

This step eliminates the occurrence of undesired checkerboard patterns as shown in Figure 2-4
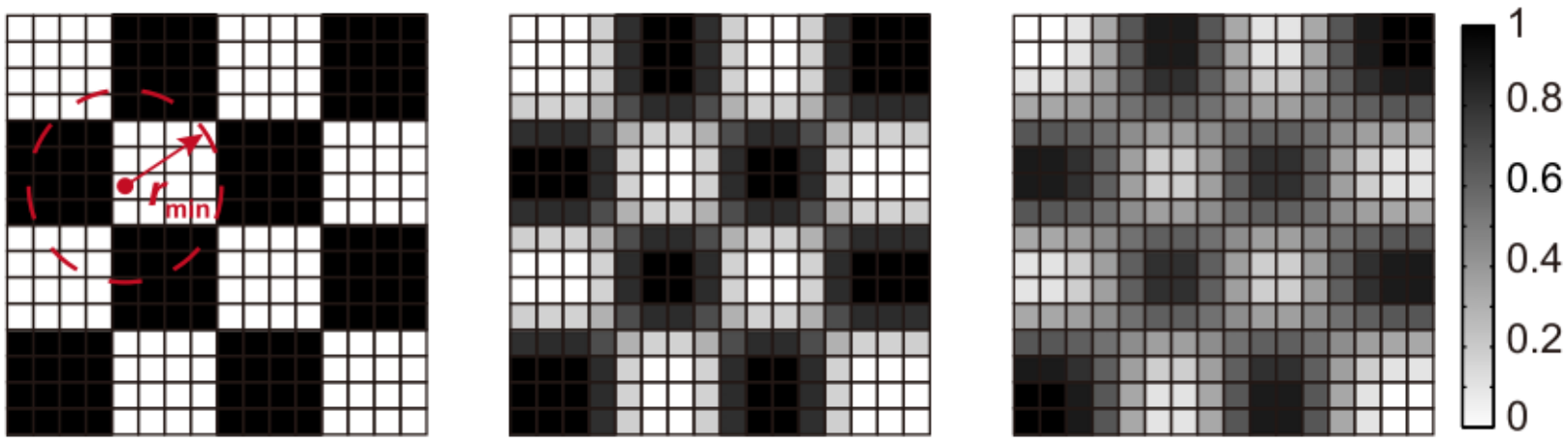

Figure 2-4 Checkerboard effect for two-phase field and the associated filtered fields [11]

In Step 3, volume fraction $\left(V_{i} / V_{0}\right.$, ) of the design is checked iteratively against $V^{*}$. At each step, if it is greater than $V^{*}$, then a new target volume, $V_{i+1}$, is computed from

$$
V_{i+1}= \begin{cases}V_{i}(1-v), & \frac{V_{i}}{V_{0}}>V^{*} \\ V^{*} V_{0}, & \frac{V_{i}}{V_{0}} \leqslant V^{*}\end{cases}
$$


After $V_{i+1}$ is computed, all elements are ranked in descending order of $\bar{\lambda}_{c}$. The first listed elements, whose total volume equals $V_{i+1}$, are marked for retention. Therefore, $\bar{\lambda}_{c}$ of the last element in this list is labelled as $\lambda_{d e l}$. Solid elements having sensitivity values below $\lambda_{d e l}$ are then marked for deletion from the design domain. Deletion is achieved by assigning the element to a void property as the TO progresses, where this void property is defined as having a significantly reduced stiffness to that of a solid element. Young's modulus of void elements in this research was defined as $1.50 \times 10^{-4}$ times that of solid elements $E_{S}$. Void elements with sensitivities above the threshold, $\lambda_{\text {add }}$, are reclassified as solid elements, bringing the volume of solid elements at $i$ to $V_{1}$. This step is skipped at the first iteration since the TO starts from a fully solid design domain. One of examples of this step is described in Figure 2-6.

where $V_{0}=$ initial volume; $\mathrm{v}=$ evolution rate; $V^{*}=$ Target volume; $V_{1}=$ Output volume at iteration 1 ;
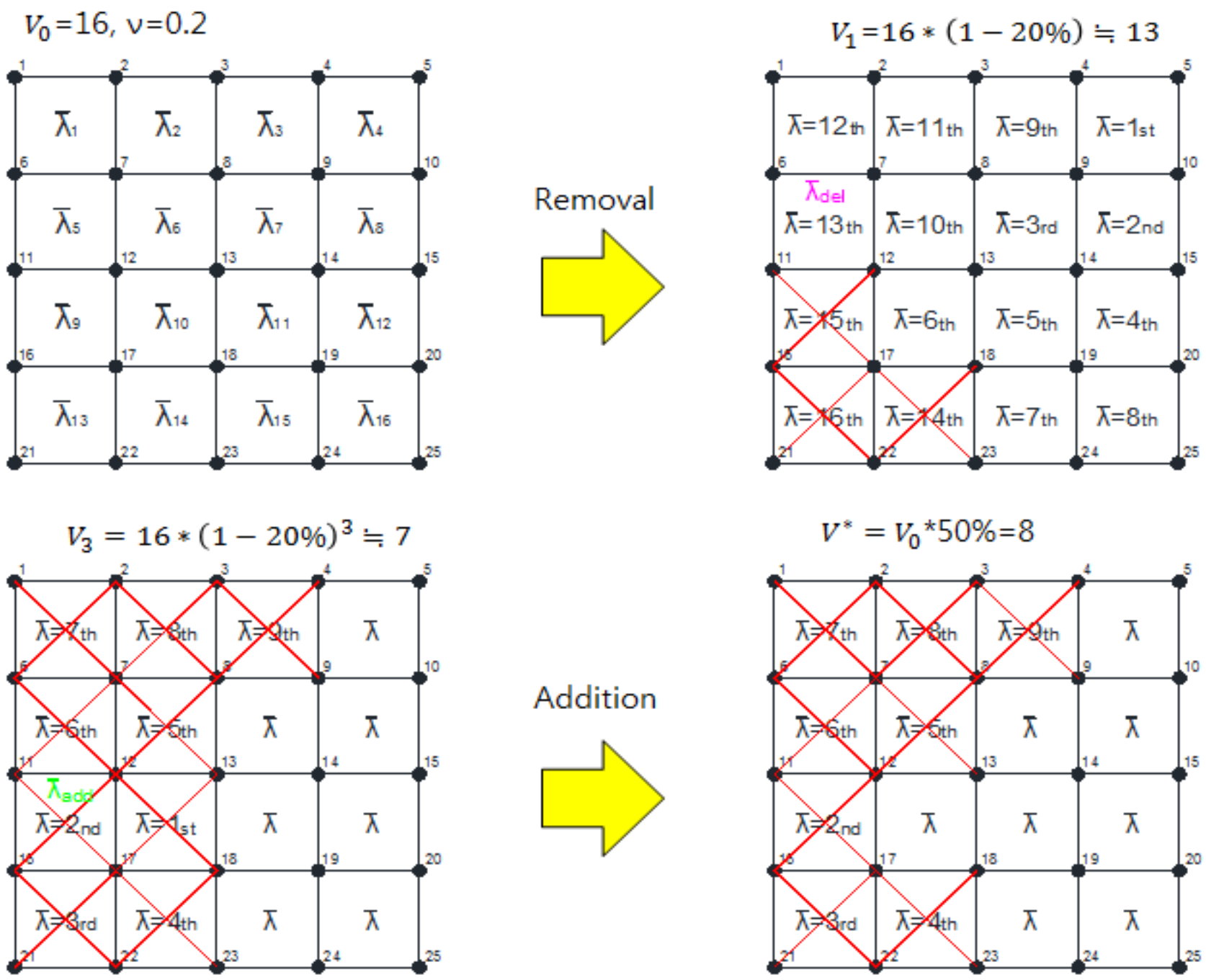

Figure 2-6 Element removal \& addition 
$V_{3}=$ Output volume at iteration 3.

Lastly, in Step 5 the TO cycle is then repeated until change of strain energy $\Delta \mathrm{C}$ is less than $\mathrm{u}$, and $V^{*}$ is obtained. $\Delta \mathrm{C}$ is computed using

$$
\Delta C=\frac{\left|\sum_{k=1}^{T}\left(C_{i-k+1}-C_{i-T-k+1}\right)\right|}{\left|\sum_{k=1}^{T} C_{i-k+1}\right|}
$$

where $T=5$, and $k$ is the sequence of integers from 1 to $T$.

a

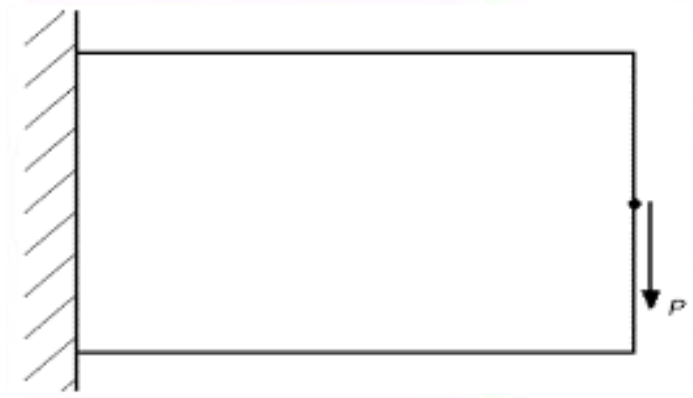

c

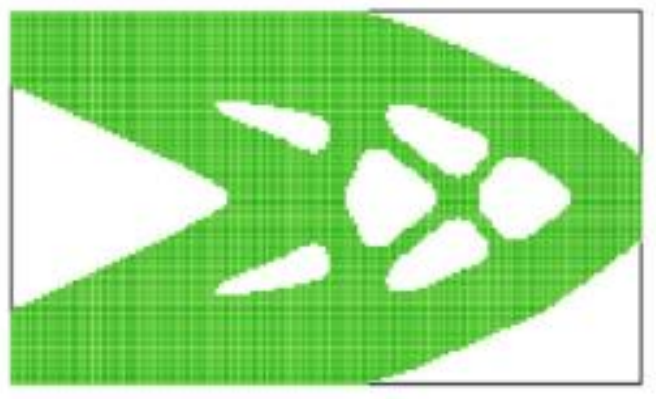

b

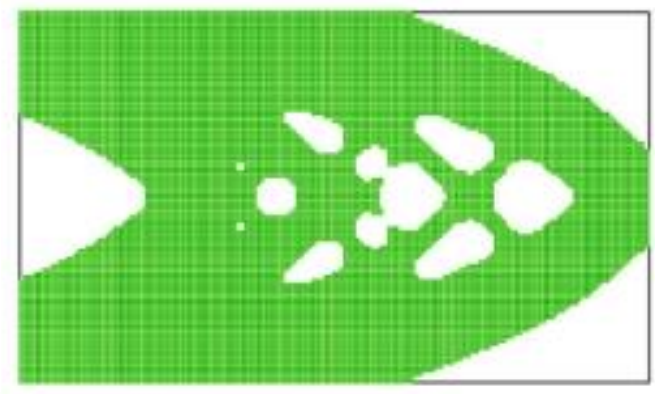

d

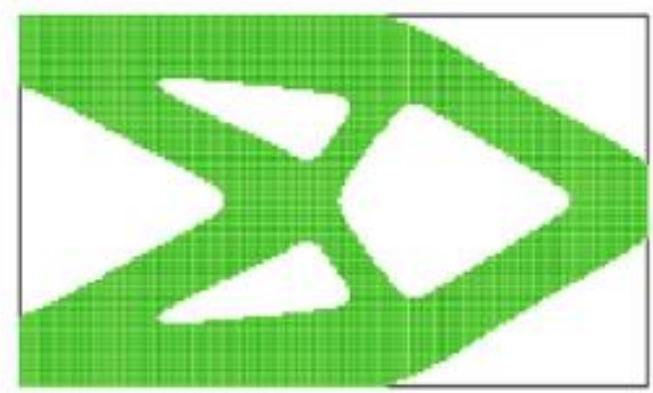

Figure 2-7 An example of BESO method (a) inital, (b) iteration 300, (c) iteration 45, (d) final topology. [16]

Figure 2-7 shows an example of BESO method for cantilever beam where the final topology is shown in Figure 2-7 (d). The BESO parameters are target volume $=50 \%$, evolution rate $=1 \%, \mathrm{R}=3 \mathrm{~mm}$ and $\Delta \mathrm{C}$ $=0.01 \%$. 


\subsubsection{Displacement-Related Structural Designs}

In mechanical engineering, for some structures such as an aircraft wing, the exterior surface should undergo minimal shape change under deformation in order to maintain the aerodynamic performance[18]. In civil engineering, some design criteria regulate the maximum displacements to guaranteed serviceability of the structure. In such cases, the displacements of a group of local nodes are of concern, the displacement limit is addressed as a global constraint. The common way of obtaining the displacement sensitivity is to apply a unit virtual load on the original model and get the displacement vector from the virtual system[17]. The displacement $k$ th component can be obtained by multiplying the displacement vector with a unit virtual load vector $\mathrm{F}^{k}$, of which the $k$ th component is unity while all other components are zero.

$$
\begin{aligned}
u^{k} & =\mathbf{F}^{k} \cdot \mathbf{u} \\
\mathbf{F}^{k} & =\{0,0, \ldots, 1,0, \ldots\}=\left\{f_{1}, f_{2}, \ldots, f_{k}, f_{k+1}, \ldots\right\} .
\end{aligned}
$$

With the virtual load $\mathrm{F}^{k}$ and the applied load $\mathrm{P}$ being constant, differentiating the $k$ th displacement component with respect to the $i$ th element and substituting $\mathrm{P}=\mathrm{KU}$ gives the following

$$
\begin{aligned}
\frac{\partial u^{k}}{\partial x_{i}}= & \frac{\partial \mathbf{F}^{k, T}}{\partial x_{i}} \mathbf{u}+\mathbf{F}^{k, T} \frac{\partial \mathbf{u}}{\partial x_{i}}=\frac{\partial \mathbf{F}^{k, T}}{\partial x_{i}} \mathbf{u} \\
& +\mathbf{F}^{k, T}\left(\frac{\partial \mathbf{K}^{-1}}{\partial x_{i}} \mathbf{P}+\mathbf{K}^{-1} \frac{\partial \mathbf{P}}{\partial x_{i}}\right) \\
= & \mathbf{F}^{k, T} \frac{\partial \mathbf{K}^{-1}}{\partial x_{i}} \mathbf{P}=-\mathbf{F}^{k, T} \mathbf{K}^{-1} \frac{\partial \mathbf{K}}{\partial x_{i}} \mathbf{u}=-\mathbf{u}^{k, T} \frac{\partial \mathbf{K}}{\partial x_{i}} \mathbf{u}
\end{aligned}
$$

where $u^{k}=K^{-1} F^{k}$ is system response in the displacement field under the unit virtual load. Substituting the material interpolation in $\mathrm{K}=\sum_{i} x_{i}^{p} E_{i}^{0}$ into the above derivative gives finally the element sensitivity for the $k$ th displacement vector with respect to the $i$ th element

$$
\alpha_{i}=\frac{\partial u^{k}}{\partial x_{i}}=-p x_{i}^{p-1} \mathbf{u}_{i}^{k, T} \mathbf{K}_{i}^{0} \mathbf{u}_{i}
$$

where $u_{i}^{k, T}$ and $u_{i}$ are the element displacement vector under the unit virtual load and the real load conditions respectively.

An example addresses the topology optimization of a cantilever shown in Figure 2-8. A uniform distributed load is applying on the top non-designable deck illustrated in dark grey. This example aims to 
minimize the volume while the maximum absolute displacement is not allowed to exceed a limit of 1.48 $\times 10^{-6} \mathrm{~m}$

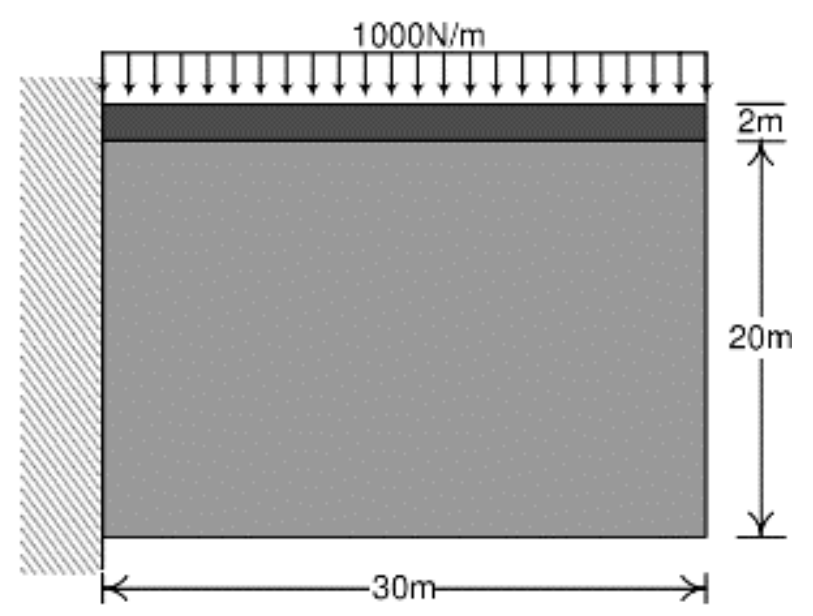

Figure 2-8 Cantilever with non-designable deck: design domain [105]

Final designs with displacement constraint and with optimal stiffness are shown in Figure 2-9 and Figure 2-10, respectively. The difference between these two final topologies is obvious by observing the deformed shapes. The two final solutions have a significant difference in the maximum displacement: with the same volume fraction, the maximum displacement of the stiffness design is $24 \%$ higher than that of the displacement design.

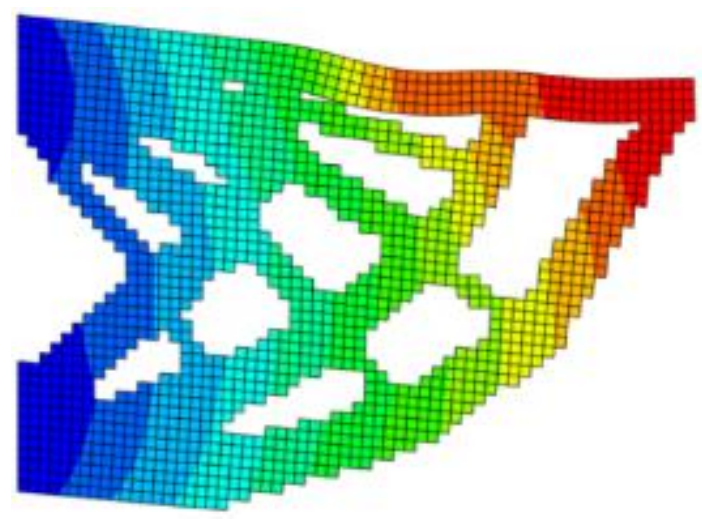

Figure 2-9 Final topology of the cantilever with displacement constraint $\left(\mathrm{V}=49.8 \%, \mathrm{~d}_{\max }=\right.$ $\left.1.478 \times 10^{-6} \mathrm{~m}\right)[105]$

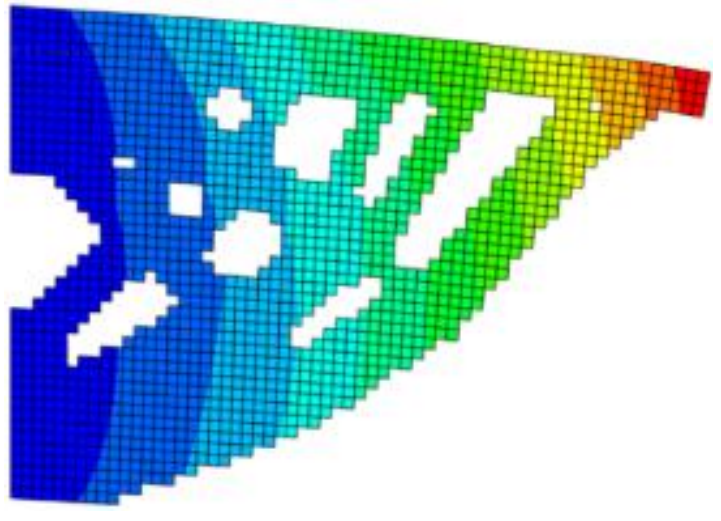

Figure 2-10 Final topology from stiffness optimal design $\left(\mathrm{V}=49.8 \%, \mathrm{~d}_{\max }=1.834 \times 10^{-6}\right.$ m) $[105]$

\subsubsection{Design of Structural Natural Frequency}

Frequency optimization is of great importance in many engineering fields e.g. aeronautical and automotive industries. Modified SIMP model using a discontinuous function has been used and applied 
successfully to solve the frequency optimization problems[19][20]. In the finite element analysis, the dynamic behavior of a continuum structure can be represented by the following general eigenvalue problem.

$$
\left(\mathbf{K}-\omega_{i}^{2} \mathbf{M}\right) \mathbf{u}_{i}=0
$$

where $\mathrm{K}$ is the global stiffness matrix and $\mathrm{M}$ is the global mass matrix. $\omega_{i}$ is the $i$ th natural frequency and $\mathrm{u}_{i}$ is the eigenvector corresponding to $\omega_{i}$. The natural frequency $\omega_{i}$ and the corresponding eigenvector $\mathrm{u}_{i}$ are related to each other by Rayleigh quotient.

$$
\omega_{i}^{2}=\frac{\mathbf{u}_{i}^{\mathrm{T}} \mathbf{K u} \mathbf{u}_{i}}{\mathbf{u}_{i}^{\mathrm{T}} \mathbf{M} \mathbf{u}_{i}}
$$

In order to maximize the $i$ th natural frequency of vibrating continuum structures, for a solid-void design, the optimization problem can be stated as

$$
\begin{array}{ll}
\text { Maximize : } & \omega_{i} \\
\text { Subject to : } & V_{1}^{*}-\sum_{i=1}^{N} V_{i} x_{i}=0 \\
& x_{i}=0 \text { or } 1
\end{array}
$$

where $\mathrm{V}_{i}$ represents the volume of an individual element and $V^{*}$ is the target volume. $\mathrm{N}$ is the total number of elements in the structure. The binary design variable $\mathrm{x}_{i}$ represents the density of the $i$ th element and small value $\mathrm{x}$ min is used to represent a void element.

The derivatives of the global mass matrix $\mathrm{M}$ and stiffness matrix $\mathrm{K}$ for the finite element analysis behind the optimization can be calculated by

$$
\begin{aligned}
& \frac{\partial \mathbf{M}}{\partial x_{i}}=\mathbf{M}_{i}^{1} \\
& \frac{\partial \mathbf{K}}{\partial x_{i}}=\frac{1-x_{\min }}{1-x_{\min }^{p}} p x_{i}^{p-1} \mathbf{K}_{i}^{1}
\end{aligned}
$$

where $M_{i}^{1}$ and $K_{i}^{1}$ are the elemental mass matrix and stiffness matrix for solid elements. According to Eq 2-11 the sensitivity of the objective function, xi can be expressed by

$$
\frac{d \omega_{i}}{d x_{i}}=\frac{1}{2 \omega_{i} \mathbf{u}_{i}^{\mathrm{T}} \mathbf{M} \mathbf{u}_{i}}\left[2 \frac{\partial \mathbf{u}_{i}^{\mathrm{T}}}{\partial x_{i}}\left(\mathbf{K}-\omega_{i}^{2} \mathbf{M}\right) \mathbf{u}_{i}+\mathbf{u}_{i}^{\mathrm{T}}\left(\frac{\partial \mathbf{K}}{\partial x_{i}}-\omega_{i}^{2} \frac{\partial \mathbf{M}}{\partial x_{i}}\right) \mathbf{u}_{i}\right]
$$

Substituting the derivatives of the matrices $\mathrm{K}$ and $\mathrm{M}$ and assuming that the eigenvector $u_{i}$ is normalized with respect to the mass matrix $\mathrm{M}$, the sensitivity of the $i$ th natural frequency for solid-void designs can 
be found as

$$
\alpha_{i}=\frac{1}{p} \frac{d \omega_{i}}{d x_{i}}= \begin{cases}\frac{1}{2 \omega_{i}} \mathbf{u}_{i}^{\mathbf{T}}\left(\frac{1-x_{\min }}{1-x_{\min }^{p}} \mathbf{K}_{i}^{1}-\frac{\omega_{i}^{2}}{p} \mathbf{M}_{i}^{1}\right) \mathbf{u}_{i} & x_{i}=1 \\ \frac{1}{2 \omega_{i}} \mathbf{u}_{i}^{\mathbf{T}}\left(\frac{x_{\min }^{p-1}-x_{\min }^{p}}{1-x_{\min }^{p}} \mathbf{K}_{i}^{1}-\frac{\omega_{i}^{2}}{p} \mathbf{M}_{i}^{1}\right) \mathbf{u}_{i} & x_{i}=x_{\min }\end{cases}
$$

In the BESO method, only two discrete values Xmin for void elements and 1 for solid elements are used.

Figure 2-11 shows an example of a 2D structure to maximize its fundamental frequency for a target volume fraction $V_{f}=50 \%[20]$.

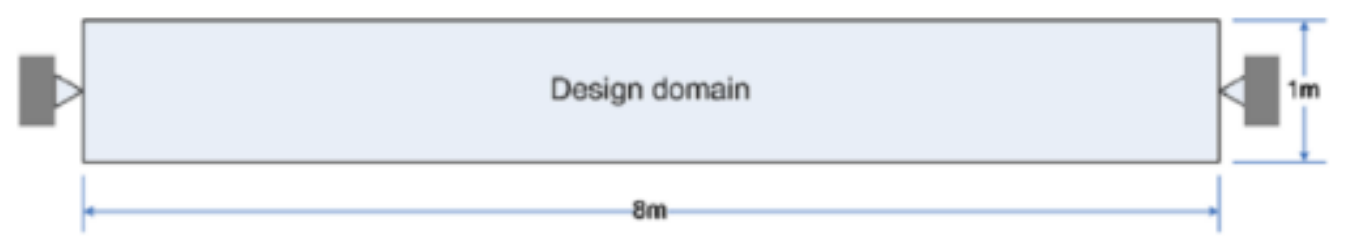

Figure 2-11 Design domain of the simply supported beam [11]

In BESO a simple way to solve this problem is to taking average of the two sensitivities[21]. The optimal design and first two eigenmodes of the optimal design are given in Figure 2-12.

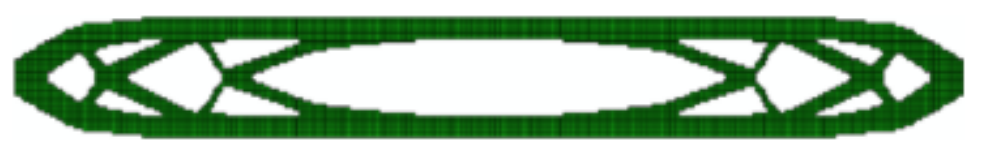

(a)

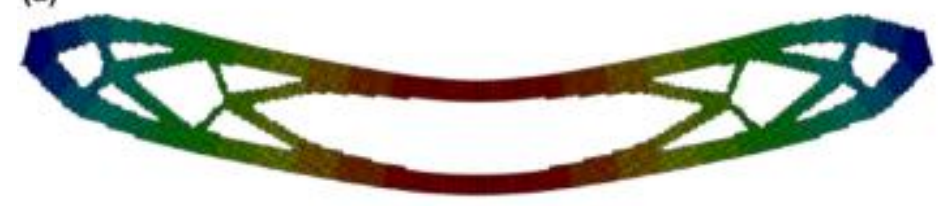

(b)

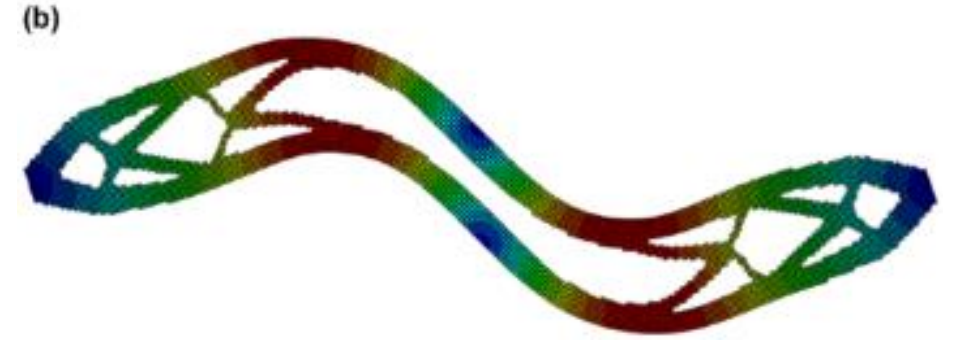

Figure 2-12 The first two eigenmodes of the optimal design: (a) the first eigenmode (b) the second eigenmode [11] 


\subsection{Shape Optimization}

In the conventional shape optimization, the adequate boundary representation and design variables are required for the success of the optimization. In the early days of shape optimization, the coordinates of the boundary nodes of a finite element model were used as design variables[22]. Using nodal coordinates as design variables is very intuitive and directly related to the finite element method. However, such design variables lead to unrealistic designs due to irregular boundaries, difficulty for maintaining adequate finite element mesh and the excessive number of design variables[23][24][25][26]. In order to guarantee the smoothness of boundaries, many researchers tried to apply polynomial functions to boundaries[27][28]. The coefficients of polynomials were used as design variables in their approaches. Although the polynomial boundary representation gives sufficient smoothness, oscillatory boundaries were observed in higher order polynomial such as Lagrange polynomial. Splines such as B-spline and NURBS could eliminate the oscillatory boundary and be locally controlled with high degree of smoothness. Thus, the spline boundary representation became the most popular geometrical representation in shape optimization.

\subsubsection{B-Splines}

The shape of a spline may be controlled by a number of control vertices. However, the degree of a Bspline is not determined by the number of control vertices. B-spline of degree $p$ with $n+1$ control vertices is defined as

$$
\boldsymbol{r}(u)=\sum_{i=0}^{n} M_{i, p}(u) \boldsymbol{V}_{i}, \quad 0 \leq u \leq 1,
$$

where the $\mathrm{p}$ th degree B-spline basis functions $M_{i, p}$ are defined as

$$
\begin{aligned}
& M_{i, 0}(u)= \begin{cases}1 & \text { if } u_{i} \leq u<u_{i+1} \\
0 & \text { otherwise, }\end{cases} \\
& M_{i, p}(u)=\frac{u-u_{i}}{u_{i+p}-u_{i}} M_{i, p-1}(u)+\frac{u_{i+p+1}-u}{u_{i+p+1}-u_{i+1}} M_{i+1, p-1}(u), \quad p \geq 1, \\
& M_{m-p-1, p}(1)=1 .
\end{aligned}
$$

The given scalars $u_{0}, u_{1}, \ldots, u_{m}$ are called knots. The number of knots, $\mathrm{m}+1$, equals $\mathrm{p}+\mathrm{n}+2$. In order 
for the curve to starts at $V_{0}$ and ends at $V_{n}$, the first $\mathrm{p}+1$ knots are put to 0 , and the last $\mathrm{p}+1$ knots are put to 1 . A knot vector $U$ containing the knots is defined as

$$
\mathcal{U}=\{\underbrace{0, \ldots, 0}_{p+1}, u_{p+1}, \ldots, u_{m-p-1}, \underbrace{1, \ldots, 1}_{p+1}\} .
$$

If the knots in the interior of the curve, i.e. those strictly greater than 0 and strictly smaller than 1 , are evenly spaced, the knot vector is said to be uniform. In Figure 2-13 the splines corresponding to two different knot vectors one uniform and the other non-uniform are plotted.

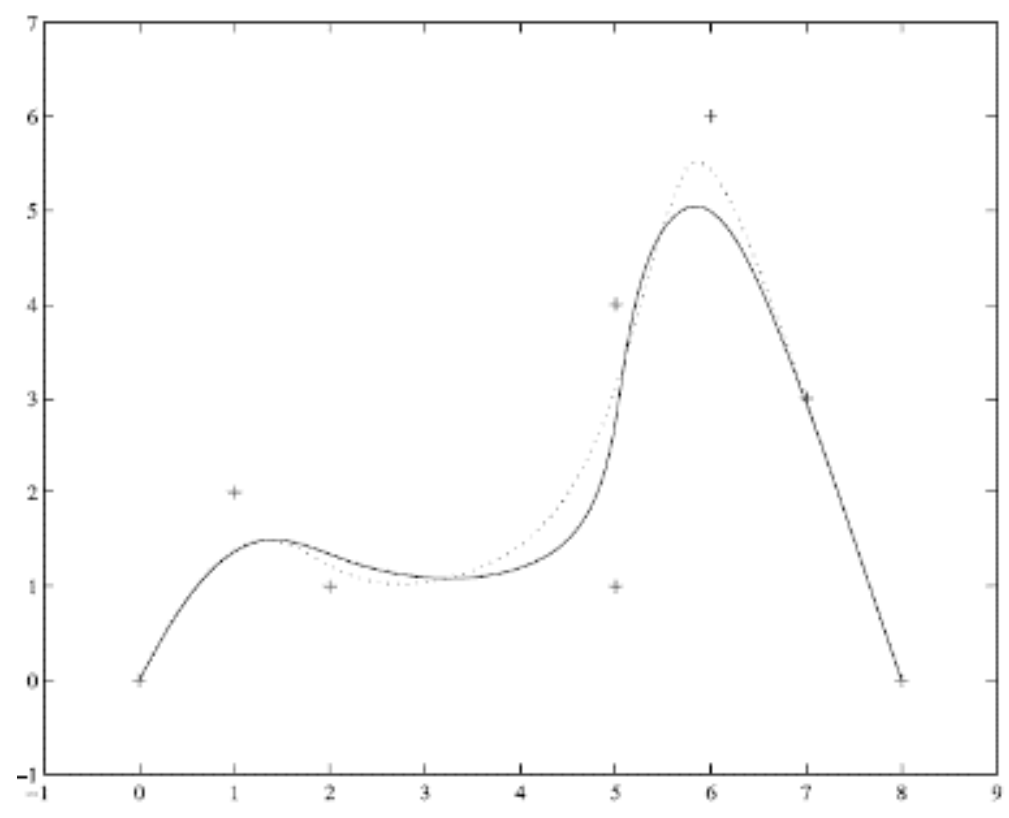

Figure 2-13 Effect of knot spacing. Solid line: uniform knot vector. Dotted line: non-uniform knot Vector [106]

\subsubsection{B-Spline Surface Mesh}

Next, a B-spline surface is defined as

$$
\boldsymbol{r}(u, v)=\sum_{i=0}^{n} \sum_{j=0}^{m} M_{i, p}(u) M_{j, q}(v) \boldsymbol{V}_{i, j}, \quad 0 \leq u \leq 1,0 \leq v \leq 1,
$$

where the B-spline basis functions $M_{i, p}$ and $M_{i, q}$ using the knot vectors 


$$
\begin{aligned}
& \mathcal{U}=\{\underbrace{0, \ldots, 0}_{p+1}, u_{p+1}, \ldots, u_{r-p-1}, \underbrace{1, \ldots, 1}_{p+1}\} \\
& \mathcal{V}=\{\underbrace{0, \ldots, 0}_{q+1}, v_{q+1}, \ldots, v_{s-q-1}, \underbrace{1, \ldots, 1}_{q+1}\}
\end{aligned}
$$

Finite element nodes are created by evaluating $\mathrm{r}(\mathrm{u}, \mathrm{v})$ for the $\mathrm{u}$-values $u_{0}^{n}, \ldots, u_{n_{u}}^{n}$, where $0=u_{0}^{n}<$ $u_{1}^{n}<\cdots<u_{n_{u}-1}^{n}<u_{n_{u}}^{n}=1$, and the v-values $v_{0}^{n}, \ldots, v_{n_{v}}^{n}$, where $0=v_{0}^{n}<v_{1}^{n}<\cdots<v_{v_{u}-1}^{n}$ $<v_{n_{v}}^{n}=1$, Each curve in the mesh corresponds to a constant value of $u$ or v. An example is shown in Figure 2-14.

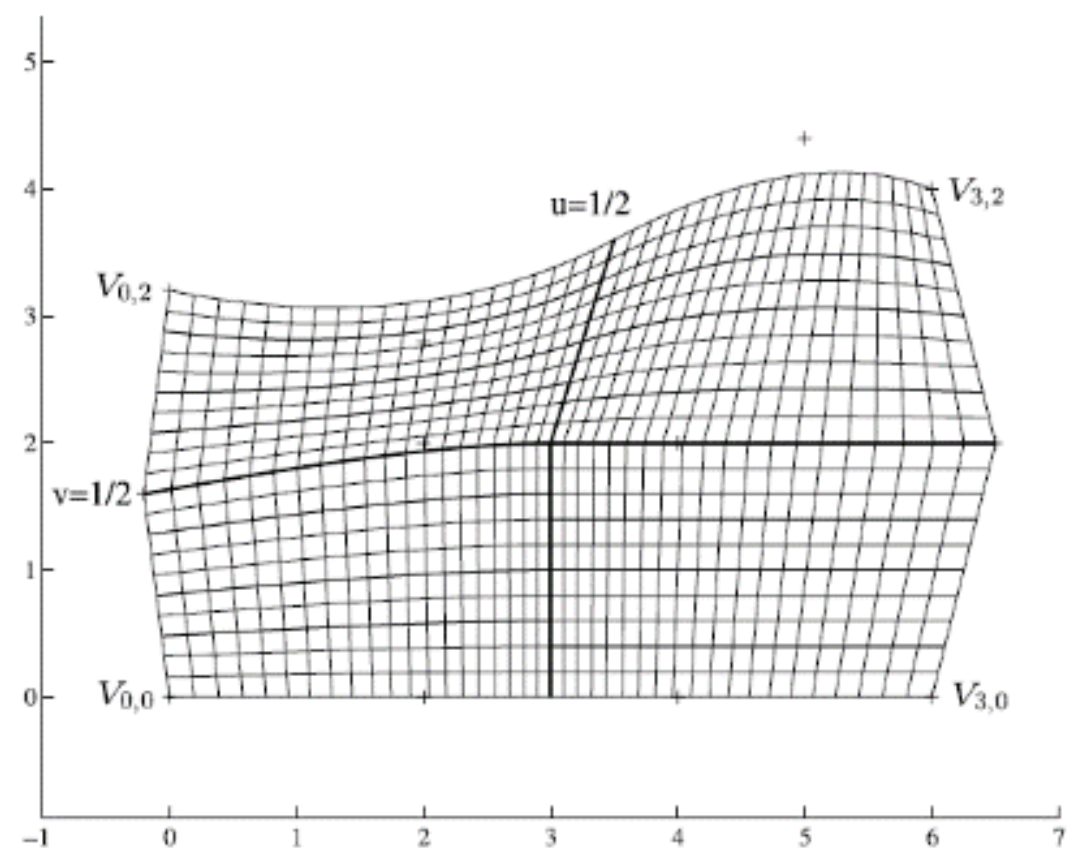

Figure 2-14 B-spline surface mesh for a case $(n=3, p=2, m=2, q=1, U=\{0,0,0,1 / 2,1,1$, $1\}, \mathrm{V}=\{0,0,1 / 2,1,1\})[107]$

\subsubsection{Sensitivity Analysis}

In shape optimization, it assumes that the shape of some boundary curves is controlled by a number of design variables $\alpha_{i}, i=1,2, \ldots, i$ as seen in Figure 2-15 

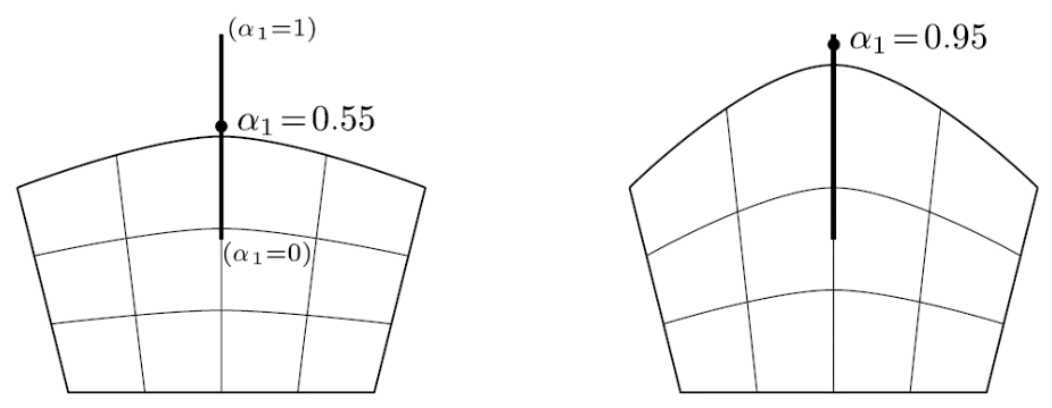

Figure 2-15 Shape optimization of a sheet. [108]

Then, the nested optimization problem may be written

$$
(\mathbb{S O})_{\mathrm{nf}} \begin{cases}\min _{\boldsymbol{\alpha}} & \hat{g}_{0}(\boldsymbol{\alpha})=g_{0}(\boldsymbol{\alpha}, \boldsymbol{u}(\boldsymbol{\alpha})) \\ \text { s.t. } & \hat{g}_{i}(\boldsymbol{\alpha})=g_{i}(\boldsymbol{\alpha}, \boldsymbol{u}(\boldsymbol{\alpha})) \leq 0, \quad i=1, \ldots, l \\ & 0 \leq \alpha_{j} \leq 1, \quad j=1, \ldots, n .\end{cases}
$$

Obtain the sensitivity of the strain displacement matrix $\mathrm{B}, \partial \mathrm{B} / \partial \alpha \mathrm{j}$ using the direct analytical method. Get the sensitivity of the element stiffness matrix and the element applied force vector from Eq 2-22:

$$
\begin{aligned}
\frac{\partial \boldsymbol{k}_{e}}{\partial \alpha_{j}} & =\int_{\hat{\Omega}}\left(\frac{\partial \boldsymbol{B}^{T}}{\partial \alpha_{j}} \boldsymbol{D} \boldsymbol{B}|\boldsymbol{J}|+\boldsymbol{B}^{T} \boldsymbol{D} \frac{\partial \boldsymbol{B}}{\partial \alpha_{j}}|\boldsymbol{J}|+\boldsymbol{B}^{T} \boldsymbol{D} \boldsymbol{B} \frac{\partial|\boldsymbol{J}|}{\partial \alpha_{j}}\right) t d \hat{\Omega} \\
\frac{\partial \boldsymbol{f}_{e}^{a}}{\partial \alpha_{j}} & =\int_{\hat{\Omega}} \boldsymbol{N}^{T}\left(\frac{\partial \boldsymbol{b}}{\partial \alpha_{j}}|\boldsymbol{J}|+\boldsymbol{b} \frac{\partial|\boldsymbol{J}|}{\partial \alpha_{j}}\right) t d \hat{\Omega} .
\end{aligned}
$$

where

$$
\begin{gathered}
\frac{\partial \boldsymbol{G}}{\partial \alpha_{j}}=-\boldsymbol{G} \frac{\partial \boldsymbol{X}}{\partial \alpha_{j}} \boldsymbol{G} \\
\frac{\partial|\boldsymbol{J}|}{\partial \alpha_{j}}=|\boldsymbol{J}| \operatorname{tr}\left(\boldsymbol{G} \frac{\partial \boldsymbol{X}}{\partial \alpha_{j}}\right)
\end{gathered}
$$

Use Eq 2-24 to calculate the required sensitivities of the objective function and the constraints:

$$
\frac{\partial \hat{g}_{i}}{\partial \alpha_{j}}=\frac{\partial g_{i}}{\partial \alpha_{j}}+\frac{\partial g_{i}}{\partial \boldsymbol{u}} \frac{\partial \boldsymbol{u}}{\partial \alpha_{j}}
$$

Figure 2-16 shows an example of B-spline mesh with $6 \times 2$ control vertices, $2^{\text {nd }}$-degree curves in the udirection, and $1^{\text {st }}$-degree curves in the v-direction. 


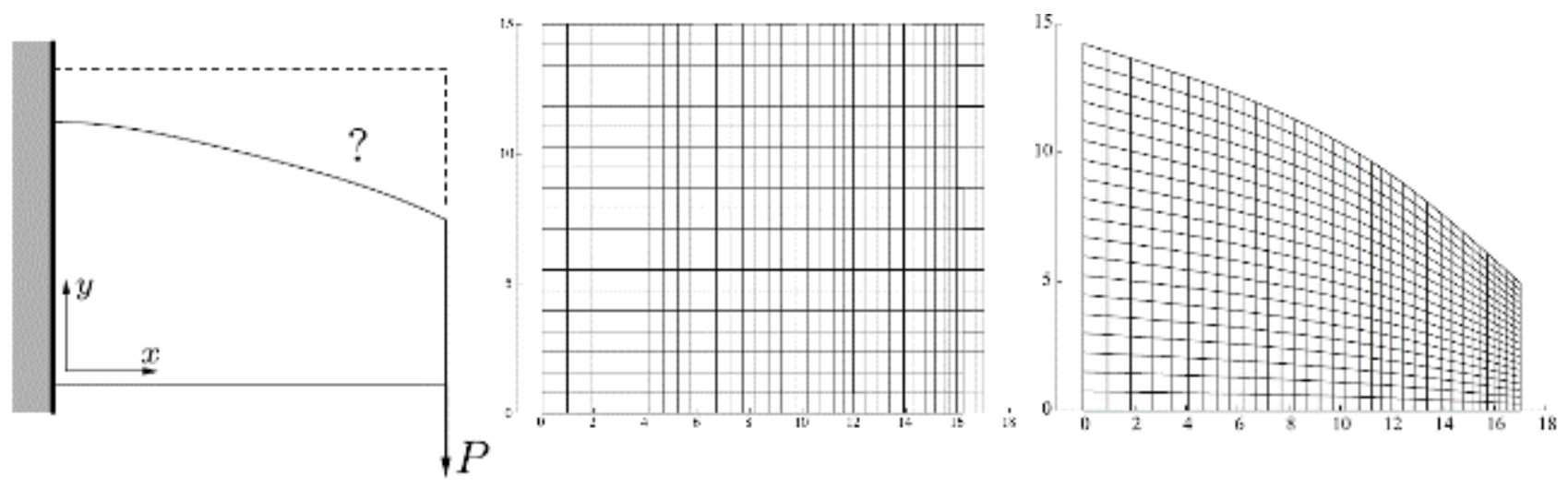

Figure 2-16 Initial mesh and optimized shape (10 iterations) of a cantilever plane sheet [108]

\subsection{Design of Fiber Reinforcement}

The DMO (Discrete Material Optimization)[29] [30], SFP (Shape Functions with Penalization)[31], and BCP (Bi-value Coding Parametrization)[32] are the most recent optimization methods for optimized discrete fiber angle selection. DMO method obtains the optimized angles through an optimization approach based on a material model formed by combining multiple elasticity tensors considering different fiber orientations. SFP is simpler than the DMO and utilizes a smaller number of design variables with fast convergence speed. However, it considers fiber angles $0^{\circ}, \pm 45^{\circ}$ and $90^{\circ}$. BCP utilizes a parameterization by using interpolation functions with penalties and it is capable of solving optimization problems subjected to constraints such as buckling load factors, limited displacement, among others. These methods were proposed as alternatives to the CFAO (Continuous Fiber Angle Optimization) which solution is highly dependent on the initial fiber configuration.[29]. In this research, DMO is applied since it is FE-based and output data from structural optimization can be used.

The classical method to find optimal orientation of orthotropic materials was to use the local orientation as design variables. As shown in Figure 2-17, each arrow represents the 1st principal material direction in a finite element framework. The design variables are then the continuous parameters, $\theta_{m}$, The optimization problem can be stated as 


$$
\begin{array}{ll}
\text { Objective : } & \min _{\boldsymbol{\theta}} C(\mathbf{u})=\mathbf{u}^{\mathrm{T}} \mathbf{p} \\
\text { Subject to : } & \mathbf{K u}=\mathbf{p} \\
& \boldsymbol{\theta}_{\min } \leqslant \boldsymbol{\theta} \leqslant \boldsymbol{\theta}_{\max }
\end{array}
$$

where $\theta \min$ and $\theta \max$ typically represent $-90^{\circ}$ and $+90^{\circ}$, respectively.

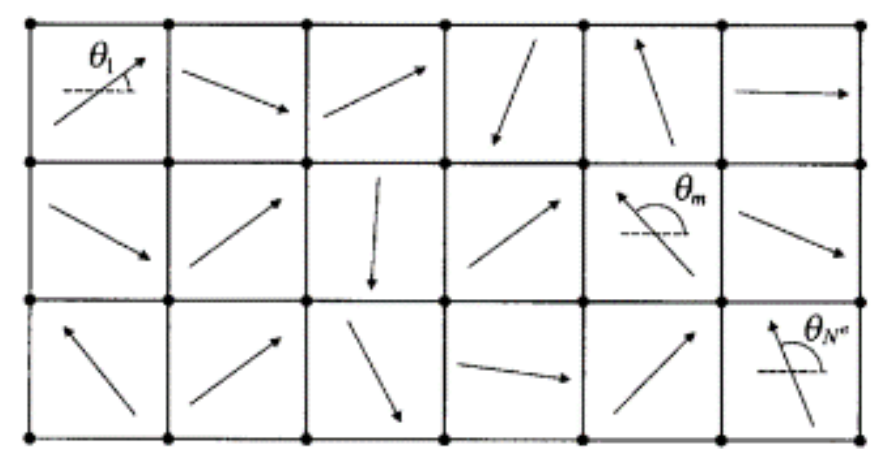

Figure 2-17 Illustration of the concept of orientation optimization in a finite element analysis [109]

The basic idea in the DMO is essentially an extension of the ideas used in structural topology optimization but instead of choosing between solid and void we want to choose between any distinct number of fiber angles. The element constitutive matrix, $C^{e}$, is expressed as a weighted sum of candidate materials, each characterized by a constitutive matrix, $C^{i}$. This can be expressed as a sum over the element number of candidate materials, $n^{e}$ :

$$
\mathbf{C}^{e}=\sum_{i=1}^{n^{e}} w_{i} \mathbf{C}_{i}=w_{1} \mathbf{C}_{1}+w_{2} \mathbf{C}_{2}+\cdots+w_{n^{e}} \mathbf{C}_{n^{e}}, \quad 0 \leqslant w_{i} \leqslant 1
$$

The single most important requirement for the DMO method is that every element must have one single weight of value 1 and all other weights of value 0 . To illustrate the methodology for fiber angle optimization solving the example in Eq 2-26 using DMO with the same orthotropic material oriented at 12 different angles $0^{\circ}, \pm 15^{\circ}, \pm 30^{\circ}, \pm 45^{\circ}, \pm 60^{\circ}, \pm 75^{\circ}, 90^{\circ}$ as the candidate materials in Figure $2-18($ a) The possible material constitutions are all combinations of the 12 candidate materials for two elements, These are marked by white triangles in Figure 2-18(b) where the obtained optimum solution 30/-45० is marked by the large black dot. This solution is the 'best fit' to the global optimum solution $24.2 /-41.6^{\circ}$ obtained with CFAO. 


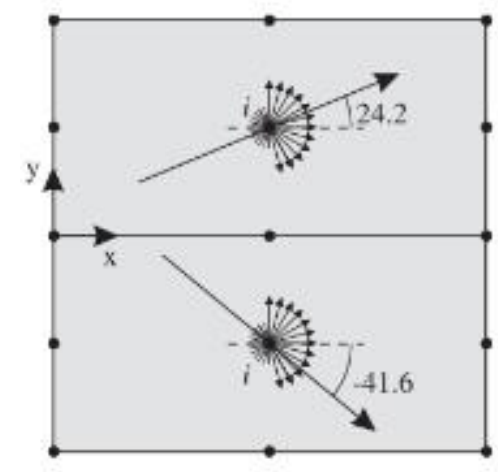

(a)
- $1.72-1.80$

घ1.64-1.72

-1.56-1.64

- $1.48-1.56$

ㅁ. $1.40-1.48$

a $1.32-1.40$

ㅁ. $1.24-1.32$

ㅁ.1.16-1.24

ㅁ. 1.08-1.16

ㅁ. $1.00-1.08$

(b)

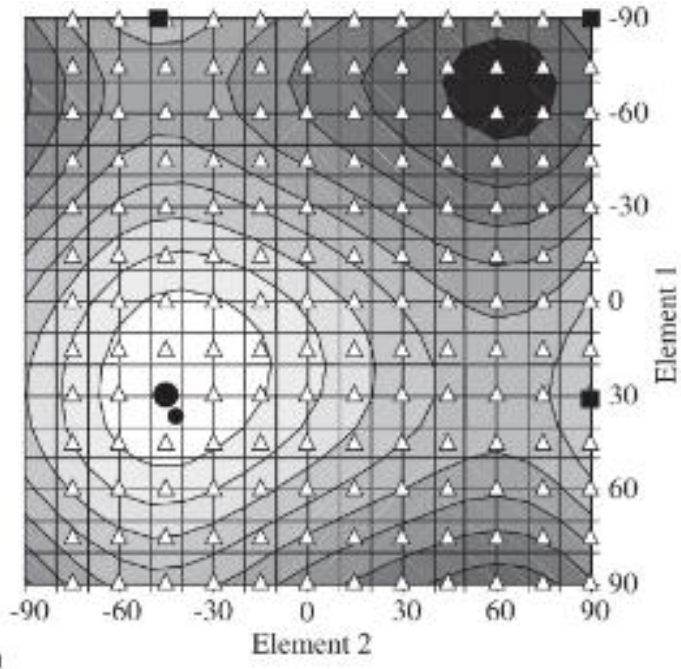

Figure 2-18 Test example objective function: (a) the candidate materials at 12 angles, i ; and (b) the white triangles mark possible combinations of candidate materials [109]

As an example, the cantilever beam with distributed top load is chosen for a standard test. The beam consists of 768 shell elements. The DMO setup 12 candidate materials in each element, it results in a model having $9216(12 \times 768)$ design variables in total.

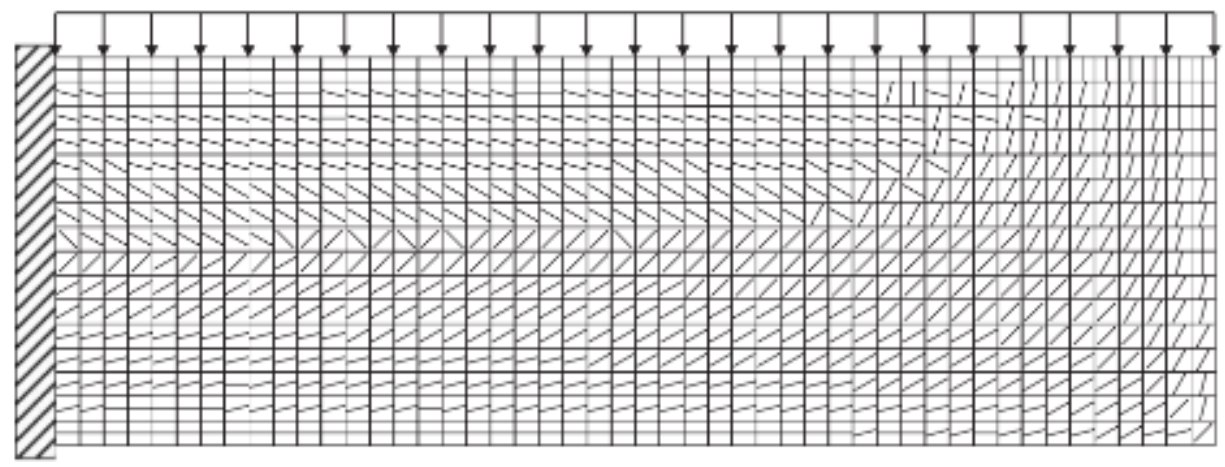

Figure 2-19 Optimal fiber angle distribution using 768 elements and a single candidate material at $\left[90, \pm 75, \pm 60, \pm 45, \pm 30, \pm 15,0^{\circ}\right][109]$

Collecting design variables in sections reduces the number of total design variables by merging several design variables from different sections and elements into a single variable. To illustrate the methodology, the cantilever problem has been solved using 48 patches of $4 \times 4$ elements, which reduces the number of design variables to 576. The resulting optimal fiber angle distribution is shown in Figure 2-20. This method ultimately allows easy setting of toolpath for individual sections in additive manufacturing. 


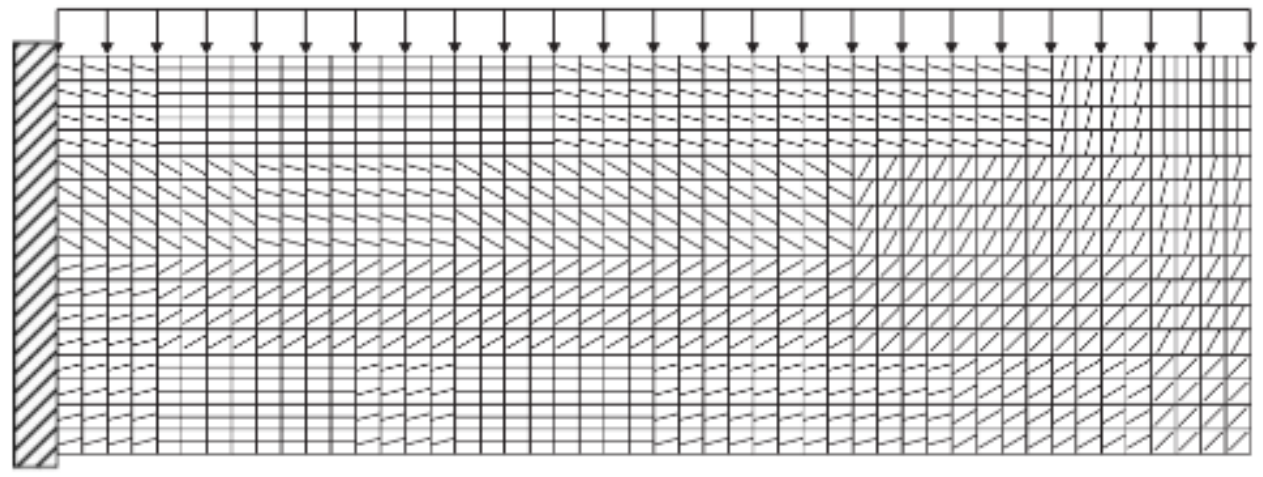

Figure 2-20 Optimal fiber angle distribution using 768 elements in 48 patches of $4 \times 4$ elements [109] 


\section{Chapter 3 ADDITIVE MANUFACTURING}

Additive manufacturing is sometimes called rapid prototyping and what is popularly called 3D Printing. The basic principle of AM technology is initially generated using a three-dimensional computer-aided design system. AM technology certainly significantly simplifies the process of producing complex 3D objects directly from CAD data.

There are couple ways to classify AM technologies. The first method is to classify according to fundamental technology such as laser or extrusion. [33][34]. Another method is to classify according to the type of raw material input such as metal or thermoplastics [35]. Recently Pham suggested a comprehensive classification method [36], which uses a two-dimensional classification method as shown in Figure 3-1

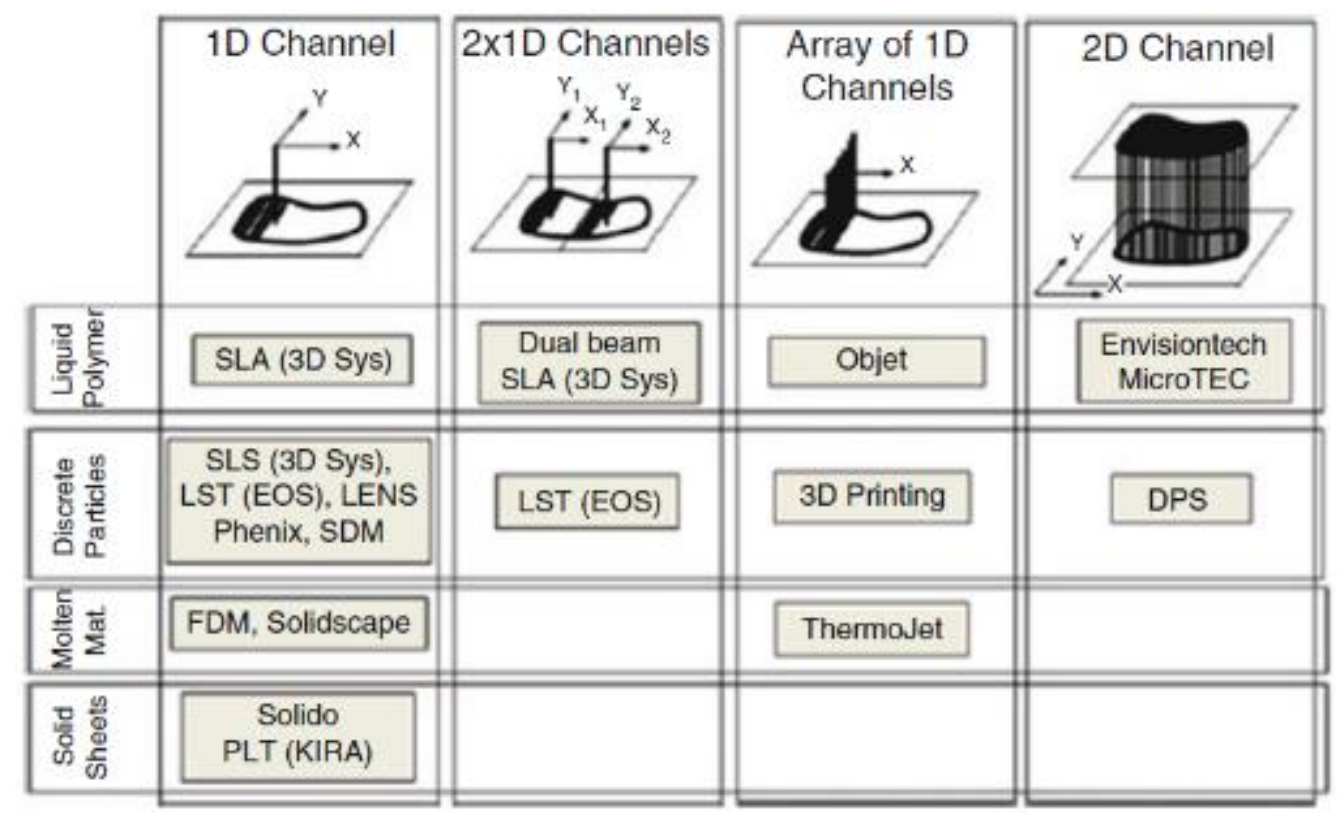

Figure 3-1 Layered manufacturing (LM) processes [110]

\subsection{Fused Filament Fabrication (FFF)}

Since RepRap was released as open-source, [37][38][39] 3D printing has been more available for people [40]. Up to now, the majority type of 3D printing system is Fused filament fabrication (FFF) [41]. Engineers are working on geometry-free manufacturing using FFF in many countries [42][43]. Previous studies have shown FFF 3D printing not only allows for a lower cost of goods for the consumer [44], but 
a lower impact on the environment as well [45]. To be specific, the system only needs filaments, this lead to not leave any wastes when compared with other manufacturing. It has been proven to be an economically beneficial purchase [46][47][48].

FFF can be visualized as similar to cake icing, the material is forced out through a nozzle when pressure is applied. Nozzle diameter will remain constant and the material being extruded must be in a semisolid state when it comes out of the nozzle. This material must fully solidify while remaining in that shape. Furthermore, the material must bond to material that has already been extruded so that a solid structure can be built. Once a layer is completed, the machine must move the part downwards, so that a further layer can be produced.

\subsubsection{Extrusion}

Extrusion in 3-D printing using material extrusion consists of cold end and hot end. The cold end is part of an extruder system that pulls and feeds the material from the spool, and pushes it towards the hot end. The cold end is mostly gear-based supplying torque to the material and controlling the feed rate by a stepper motor. Figure 3-2 illustrates typical extruder system of FFF-based 3D printer. The hot end is the active part which also melts the filament. It allows the molten plastic to exit from the small nozzle to form a thin bead of plastic. Typical size of diameter is ranging from $0.3 \mathrm{~mm}$ to $1.0 \mathrm{~mm}$. Different types of nozzles and heating methods are highly dependent on the material to be printed.

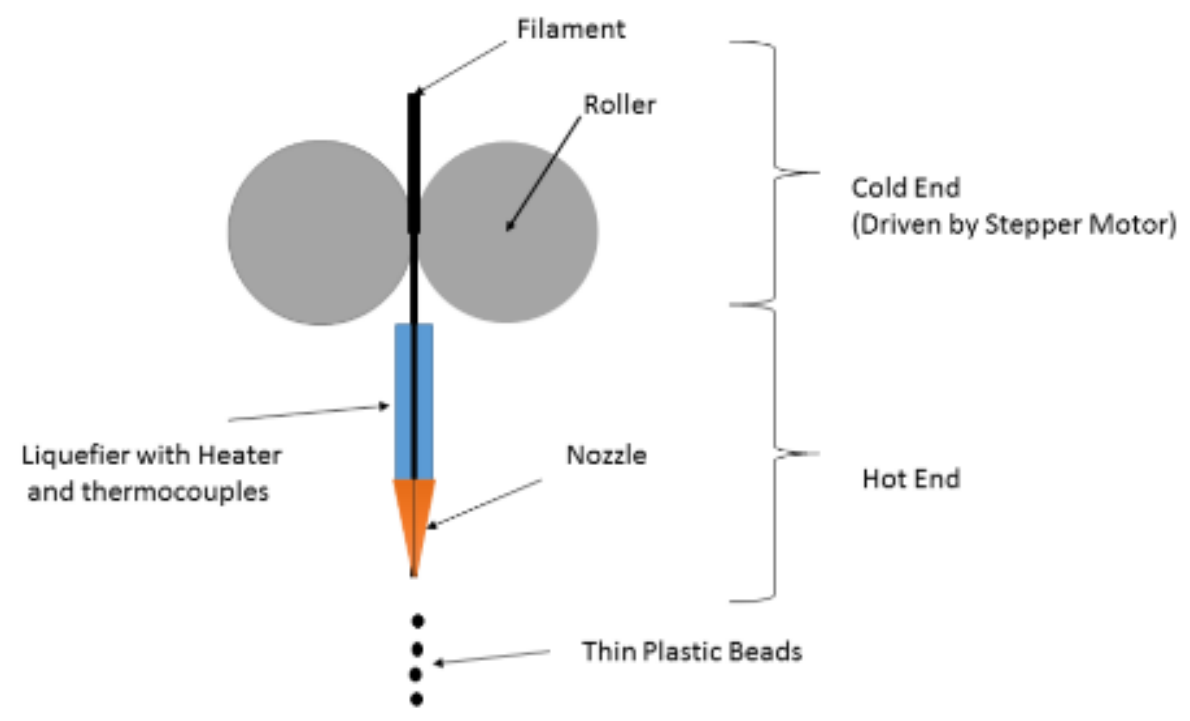

Figure 3-2 FFF based-3D printer Extruder [110] 


\subsubsection{Limitations of FFF}

There are some disadvantages of FFF in terms of build speed, accuracy, and material density. FFF layer thickness normally is $0.1 \mathrm{~mm}$, this level of precision leads to longer build times. Also, shape of nozzles is circular, it is impossible to draw sharp external corners. Lastly, products built by FFF exhibit anisotropic mechanical properties. This has been proven not to be huge for thermoplastic polymer materials [49], but in almost every case the strength in the z-direction is less than the strength in the $\mathrm{x}-\mathrm{y}$ plane. Thus, for parts which undergo stress in a particular direction it is best to build the part such that the major stress axes are aligned with the $\mathrm{x}-\mathrm{y}$ plane.

\subsection{Carbon Fiber Reinforced Polymer}

Researchers have attempted to mix different types of fillers into the polymer matrix to improve material properties. These fillers include Titanium Dioxide [50], Jute Fibers [50], metal [51], glass fibers [52] thermotropic liquid crystalline polymer fibrils [53], vapor-grown carbon fiber [54], graphene nanoplatelets [55] and continuous fibers [56][57]. Another viable candidate is short carbon fibers. It is well known that short carbon fibers blended with unfilled thermoplastic polymers significantly improve the strength of the polymer material. These filaments are now commercially available by manufacturers such as CarbonX, Matterhackers, and ColorFabb. Figure 3-3 illustrates a printing extrusion of short fiber reinforced filament.

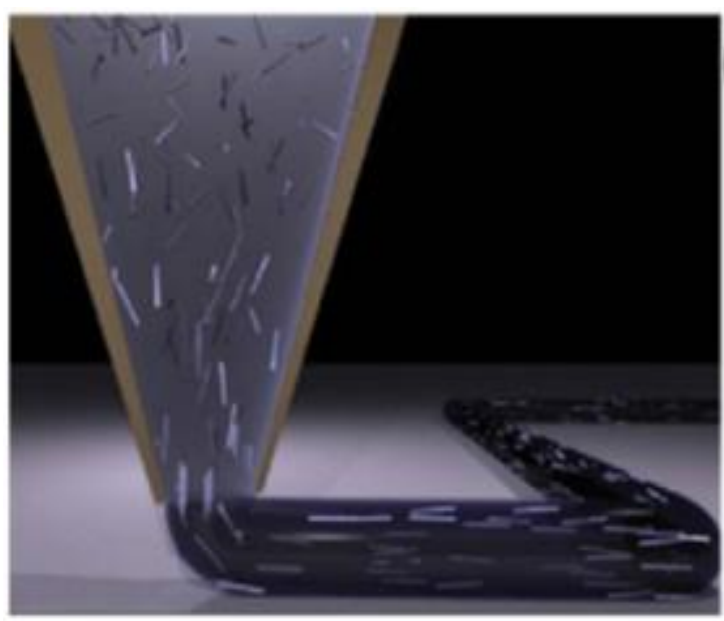

Figure 3-3 Short fiber alignment during the extrusion process [111] 


\subsubsection{Fiber Orientation Distribution}

The fiber orientation distributions have been measured experimentally using various methods. The polished cross-sections have been most commonly used to determine the distribution in the past decades. Fakirov et al. [58] measured the average angle of the fibers with respect to the flow direction using micrographs of the polished cross-section of samples. In their research, they assumed a two dimensional fiber distribution in the case of injection-molded polyethylene terephthalate. Vincent et al. [59] measured the scalar orientation factor in a molded disk of a polyamide using optical microscopy. Fischer et al. [60] used an image analyzer to measure the orientation factors of polished cross-sections of samples with 3D dimensions combining the in-plane and out-plane directions. They demonstrated that the degree of out-of-plane orientation was small. Tucker et al. [61] measured angles of fibers perpendicular to the section plane. Using the projected area, fiber orientation could be determined by second-order tensor. Zak et al. [62] used a two-section-based method for determining the threedimensional (3D) fiber orientation distribution. Eberhardt et al. [63] used a confocal laser scanning microscopy to measure the fiber orientation distributions in composites. Then they compared them with the distribution obtained using 2D image analysis. McGee et al. [64] utilized radiography method to obtain high-contrast primary images with 2D fiber orientation. This method worked for composites with low filler content, or for thin sections of high-filler-content composites. Kim et al. [65] analyzed X-ray images to determine the fiber orientation distribution of composites. The approximately $94 \%$ of precision was achieved. Also, the method was not useful for thick fiber composites. Shen et al. [66] measured the fiber orientation in $5 \mathrm{wt} \%$ short-glass-fiber-reinforced phenolic foam using micro-computed tomography (CT). 3D fiber distribution of the foam was constructed from the obtained micro-CT images, and AutoCAD was used to determine the spatial length and orientation of the individual fibers based on the imported 3D fiber distribution information. However, this technique had difficulties to be applied to high fiber content composites. Although these methods have been used in the past decades, problems to find fiber orientation distribution clearly in CFRP composites still remain unsolved. It is difficult to distinguish fibers from resin, clearly on X-ray CT images, because carbon fibers and resin have similar radiodensities. Djukic et al. [67], [68] tried to improve the contrast between carbon fibers and resin by impregnating the fibers by coating the carbon fibers with metal. However, it was reported that these techniques affect the microstructure of the composites. Scott et al. [69] used synchrotron radiation computed tomography to obtain extremely clear section images. In their research, the influence of voids on damage was evaluated. 
Although this technique can successfully distinguish fibers from resin, synchrotron accelerator is very expensive and not easy to use it.

Tekinalp et al investigated fiber orientation measurements of composites manufactured by FFF-based 3D printer [70]. The method used by Bay and Tucker [61] was followed to characterize the fiber orientation of samples. Samples were fabricated with different weight $\%$ of fiber. For the comparison, compression molded samples were tested as well. Components of second-order orientation tensors for each sample are given in Table 3-1

Table 3-1 Components of the second-order orientation tensor of ABS/CF composites [70]

\begin{tabular}{lcccccc}
\hline Carbon Fiber (wt\%) & $\mathrm{a} 11$ & $\mathrm{a} 12$ & $\mathrm{a} 13$ & $\mathrm{a} 22$ & $\mathrm{a} 23$ & $\mathrm{a} 33$ \\
\hline \multicolumn{2}{l}{ Compression-molded(CM) samples } \\
10 & 0.241 & -0.023 & 0.042 & 0.03 & 0.084 & 0.729 \\
20 & 0.493 & -0.059 & -0.054 & 0.023 & 0.046 & 0.484 \\
30 & 0.454 & -0.034 & 0.062 & 0.023 & 0.064 & 0.523 \\
40 & 0.386 & -0.043 & -0.049 & 0.036 & 0.095 & 0.578 \\
& & & & & & \\
FFF-printed samples & & & & & & \\
10 & 0.055 & 0.005 & 0.038 & 0.03 & 0.127 & 0.915 \\
20 & 0.064 & 0.004 & 0.024 & 0.028 & 0.121 & 0.909 \\
30 & 0.06 & -0.002 & -0.006 & 0.039 & 0.143 & 0.901 \\
40 & 0.093 & -0.005 & -0.018 & 0.038 & 0.139 & 0.869 \\
\hline
\end{tabular}

Components a11, a22, and a33 show orientation in the direction of $\mathrm{x} 1, \mathrm{x} 2$, and $\mathrm{x} 3$, respectively as seen in Figure 3-4. The dominant orientation tensor components for CM samples are a33 and a11. In contrast, the dominant component of the orientation tensor for FFF samples is only a33, and its nearly 1.0 value indicates that practically all fibers are oriented in the $\mathrm{x} 3$-direction. From a mechanical performance point of view, orientation in the $\mathrm{x} 3$-direction is of most interest because it is the load-bearing direction. These results emphasize the inherent characteristic of gaining high orientation by FFF process. Because of its nature, the FFF process produces samples not only with higher fiber orientation, but also with higher molecular orientation in matrix materials such as thermoplastics compared with CM and injection molding. 


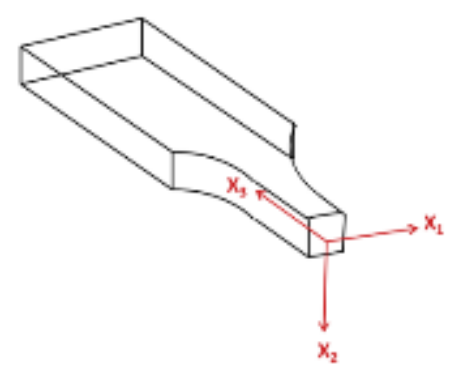

Figure 3-4 Sketch of a dog-bone sample showing orientation directions [70]

\subsubsection{Tensile Properties}

Tensile property is generally the most representative information on mechanical properties of materials.

Since carbon fiber-reinforced filament was developed, tensile properties of carbon fiber reinforced polymer have been investigated by many researchers, as shown Table 3-2.

Table 3-2 Previous tensile tests of composites produced by FFF and injection molding (IM)

\begin{tabular}{|c|c|c|c|c|}
\hline Authors, year & Matrix & Reinforcement & Process & Comments \\
\hline Zhong et al., 2001 [52] & ABS & GF short & FFF, IM & $\begin{array}{l}\text { Tests fiber weight fractions }(15 \mathrm{e} 20 \%) \text {. } \\
\text { Unidirectional tests in two perpendicular axes }\end{array}$ \\
\hline Ahn et al., 2002 [71] & ABS & - & FFF, IM & $\begin{array}{l}\text { Unidirectional tests in two perpendicular axes } \\
\text { Tests influence of FFF parameters }\end{array}$ \\
\hline $\begin{array}{l}\text { Bellini and Güçeri, } 2003 \\
\text { [72] }\end{array}$ & ABS & - & FFF & \\
\hline Shofner et al., 2003 [54] & ABS & $\begin{array}{c}\text { CF short } \\
\text { (FL } 100 \mathrm{~mm})\end{array}$ & FFF & \\
\hline Tekinalp et al., 2014 [70] & ABS & $\begin{array}{l}\text { CF short } \\
\text { (FL } 200 \text { to } 400)\end{array}$ & FFF, IM & Tests fiber weight fractions $(10 \mathrm{e} 40 \%)$ \\
\hline Love et al., 2014 [73] & ABS & CF short & FFF & Tests properties out of printing plane \\
\hline Ning et al., 2015 [74] & ABS & $\begin{array}{l}\text { CF short } \\
\text { (FL } 100 \text { and } 150)\end{array}$ & FFF & $\begin{array}{l}\text { Evaluates several material constants and } \\
\text { fiber weight fractions }(3 \mathrm{e} 15 \%) \text {. }\end{array}$ \\
\hline Ning et al., 2016 [75] & ABS & CF short & FFF & $\begin{array}{l}\text { Tests influence of FFF parameters on } \\
\text { properties }\end{array}$ \\
\hline Melenka et al., 2016 [76] & Nylon & Kevlar cont. & FFF & Uses a process adapted from FFF. \\
\hline Klift et al., 2016 [57] & Nylon & CF cont. & IM & Tests different fiber volume fractions \\
\hline $\begin{array}{l}\text { Anwer and Naguib, } 2016 \\
\text { [77] }\end{array}$ & PLA & $\begin{array}{l}\text { CF nano } \\
(\mathrm{FL}<4 \mathrm{~mm})\end{array}$ & $\mathrm{IM}$ & $\begin{array}{l}\text { Tests weight fractions }(1 \mathrm{e} 15 \%) \text {, thermal } \\
\text { and dynamical properties. }\end{array}$ \\
\hline
\end{tabular}




\begin{tabular}{|c|c|c|c|c|}
\hline $\begin{array}{l}\text { Jaszkiewicz et al., } 2016 \\
\text { [78] }\end{array}$ & PLA & GF and NF short & FFF & $\begin{array}{l}\text { Investigates manufacturability of short NF and } \\
\text { GF. }\end{array}$ \\
\hline Li et al., 2016 [79] & PLA & CF cont. & FFF & Uses a new process adapted from FFF. \\
\hline Tian et al., 2016 [80] & PLA & CF cont. & FFF & $\begin{array}{l}\text { Uses a process adapted from FFF, investigates } \\
\text { several parameters }\end{array}$ \\
\hline Tian et al., 2017 [81] & PLA & CF cont. & FFF & $\begin{array}{l}\text { Uses a process adapted from FFF w/recycled } \\
\text { CF. Evaluates impact properties. }\end{array}$ \\
\hline Yao et al., 2017 [82] & PLA & CF cont. & FFF & Investigates $\mathrm{CF}$ in structural-health monitoring \\
\hline Ferreia et al., 2017 [49] & PLA & CF short. & FFF & $\begin{array}{l}\text { Unidirectional tests in two perpendicular axes } \\
\text { Shear properties and Poisson ratios }\end{array}$ \\
\hline
\end{tabular}

In reference [49], a PLA reinforced with short carbon fibers were experimentally characterized by performing ASTM 638 and ASTM D3518 as shown Figure 3-5 and Figure 3-6, respectively. This composite has a weight fraction of $15 \%$ of carbon fibers whose length was estimated in about $60 \mathrm{~mm}$.
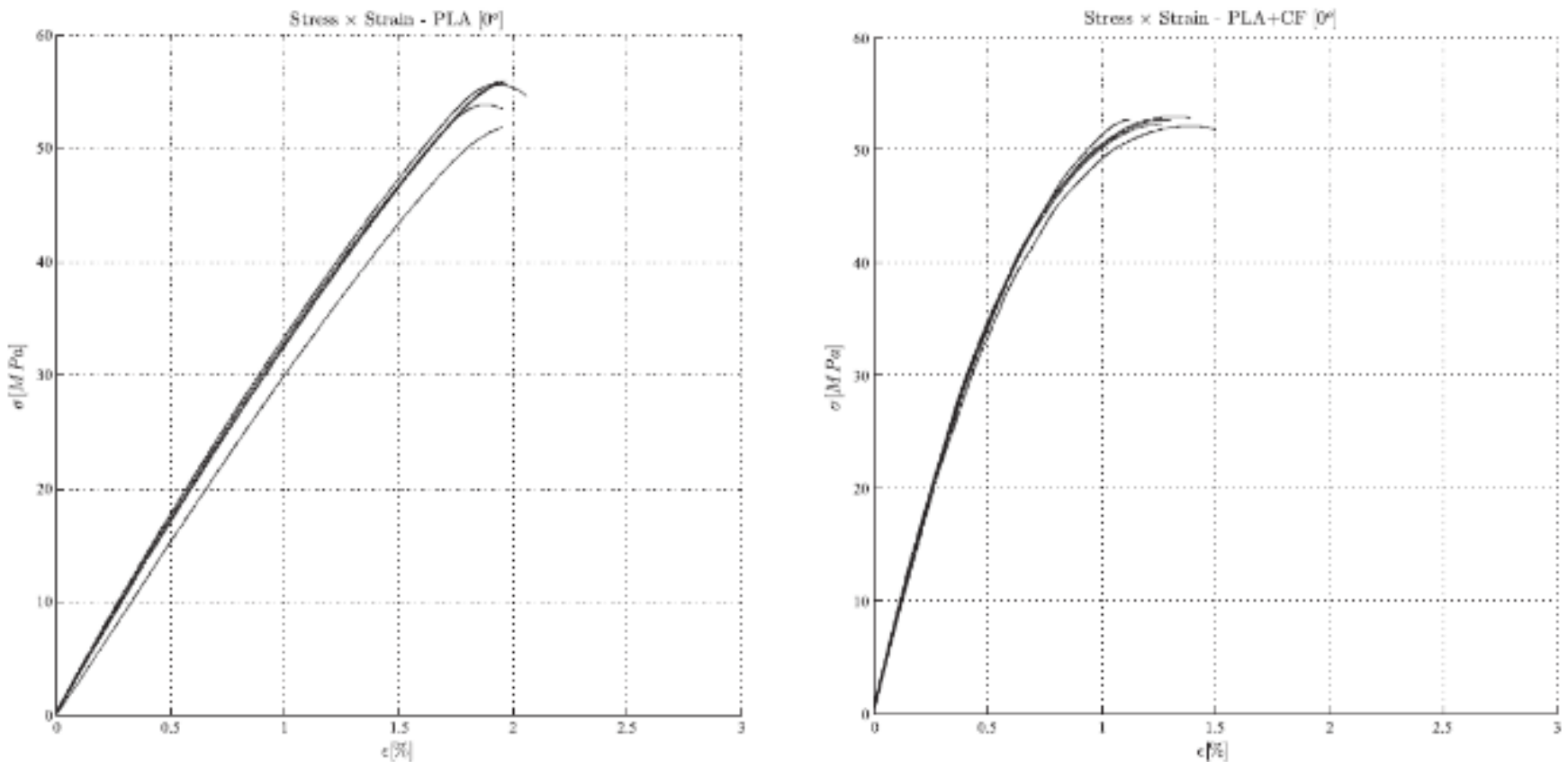

Figure 3-5 Stress vs strain data for PLA and PLA+CF printed at $0^{\circ} \quad[49]$ 

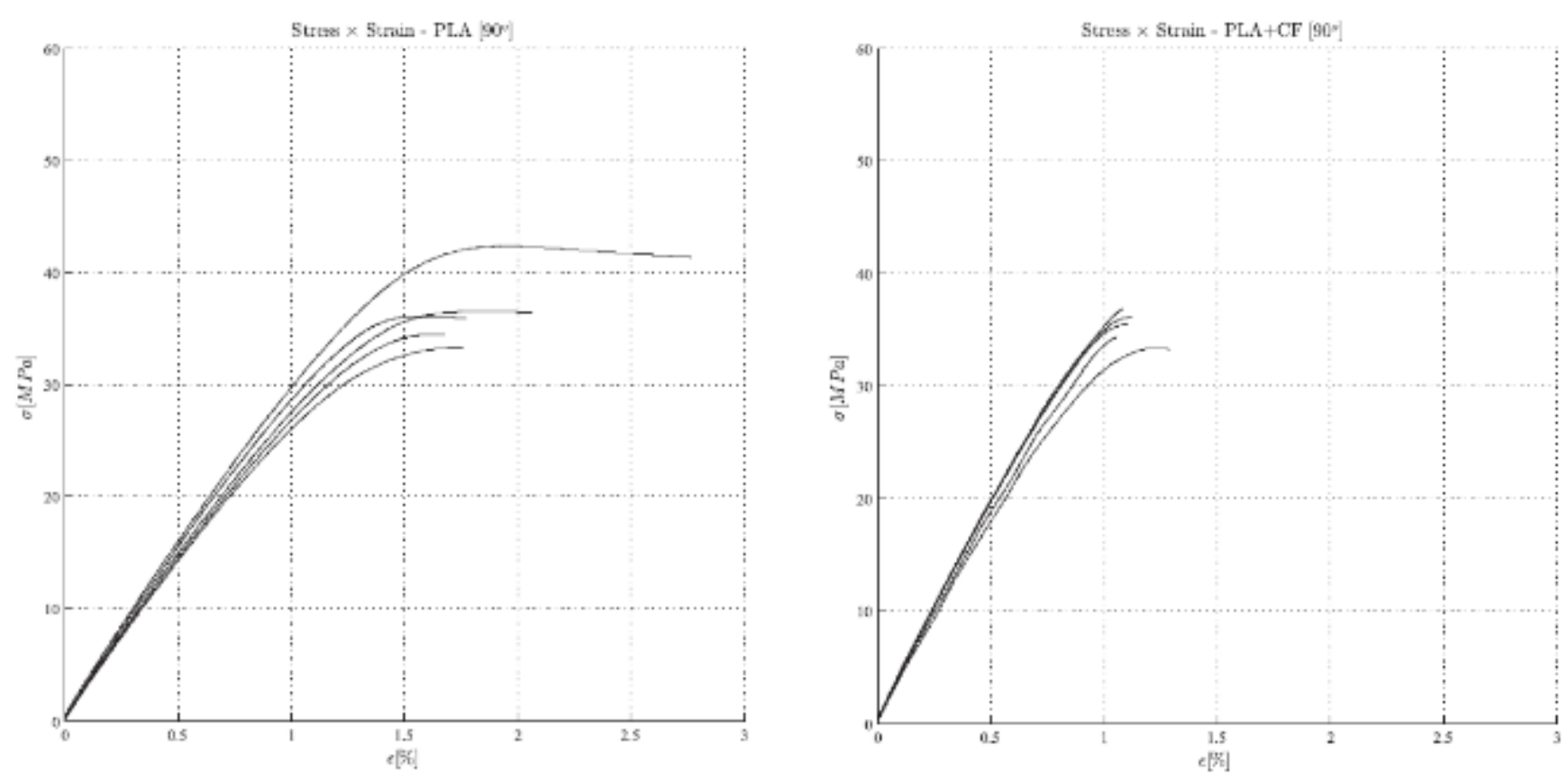

Figure 3-6 Stress vs strain data for PLA and PLA+CF printed at $90^{\circ}$ [49]

From the results in Table 3-3, the average value of E1 tensile modulus for the PLA $+\mathrm{CF}$ was more than twice (2.2 times) higher than the same property for the PLA. The E2 tensile modulus for the PLA+CF was about 1.25 times higher than the same property for the PLA, a difference not as big as found for E1. These results show that short carbon fibers provided the highest increase in stiffness for the PLA+CF tested in the printing direction. Besides, it is interesting to notice that E1 for the PLA is only slightly higher than E2 for the same material. Therefore, for the PLA, the printing orientation did not influence the material stiffness that much.

Table 3-3 Mechanical properties of PLA and PLA+CF [49]

\begin{tabular}{|c|c|c|c|c|c|c|c|c|}
\hline \multirow[t]{2}{*}{ Property } & \multirow[t]{2}{*}{ Direction } & \multicolumn{3}{|l|}{ PLA } & \multicolumn{3}{|c|}{$\mathrm{PLA}+\mathrm{CF}$} & \multirow{2}{*}{$\begin{array}{l}\text { ASTM } \\
\text { Standard }\end{array}$} \\
\hline & & Max. & Avg. & Dev. & Max. & Avg. & Dev. & \\
\hline Tensile & $0^{*}\left(E_{1}\right)$ & 3596 & 3376 & 212 & 7665 & 7541 & 96 & D638 \\
\hline Modulus (MPa) & $90^{*}\left(E_{2}\right)$ & 3340 & 3125 & 148 & 4145 & 3920 & 167 & \\
\hline $\begin{array}{l}\text { In-plane Shear } \\
\text { Modulus (MPa) }\end{array}$ & $\pm 45^{\circ}\left(G_{12}\right)$ & 1140 & 1092 & 36 & 1270 & 1268 & 5 & D3518 \\
\hline Tensile & $0^{*}\left(S_{i}^{*}\right)$ & 56.1 & 54.7 & 1.9 & 53.7 & 53.4 & 0.2 & D638 \\
\hline Strength (MPa) & $90^{*}\left(S_{2}^{*}\right)$ & 42.9 & 37.1 & 3.5 & 37.0 & 35.4 & 1.5 & \\
\hline $\begin{array}{l}\text { In-plane Shear } \\
\text { Strength (MPa) }\end{array}$ & $\pm 45^{\circ}\left(S_{12}^{*}\right)$ & 18.3 & 18.0 & 0.8 & 19.6 & 18.9 & 0.8 & D3518 \\
\hline
\end{tabular}

In reference [70], tensile strength and modulus of dog-bone specimens prepared by both FFF and CM methods were measured as shown in Figure 3-7. The results show that tensile strength increases with 
increasing fiber content in both processes. The standard deviations in tensile strength measurements for the FDM samples were significantly lower than those for the CM samples. This result suggests that the FDM process not only increases the orientation of the polymer, but also improves fiber uniformity. The increase in fiber content doesn't increase much in tensile strength at higher fiber loadings as shown Figure 3-7(a). Figure 3-7(b) shows the Young's modulus measurements of all samples. Differently with tensile strength, the moduli of FDM and CM samples overlap and increase almost linearly with increasing fiber content. The modulus value of the CM composite is increased by nearly an order of magnitude at $40 \mathrm{wt} \%$ fiber loading.
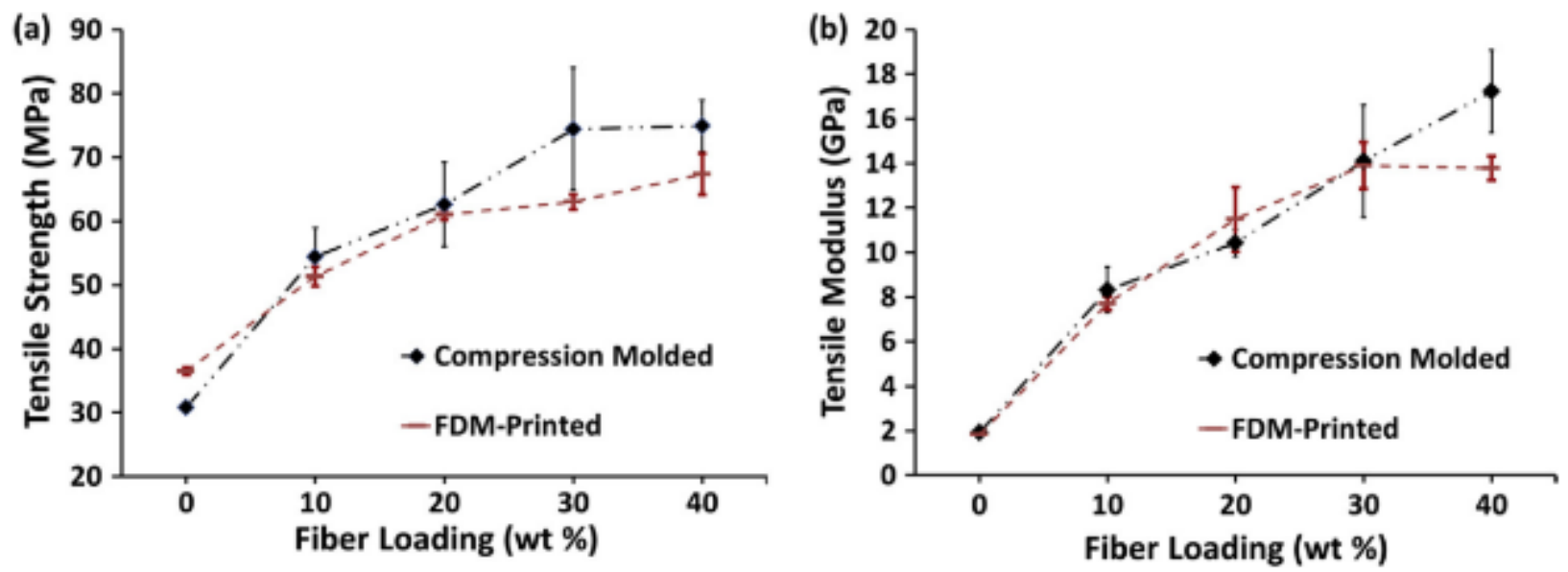

Figure 3-7 Effect of fiber content and preparation process on (a) tensile strength, and (b) modulus, of $\mathrm{ABS} / \mathrm{CF}$ composites. [70]

\subsection{Density}

The density of an additive manufactured part is crucial for its mechanical properties. For FFF parts, printing in the proper orientation with beads as close together as possible throughout the entire part is required to achieve stronger parts. These highly dense parts are expected to be stronger than parts printed by regular filled pattern on the inside. But even a part printed with beads alongside each other can vary in density. This density is determined by the voids between adjacent beads. The density defined within this study is called solidity ratio (SR). Figure 3-8 shows an illustration of a printed parts' cross-section as well as its expansion. SR determines the porosity or solidity of the part. The SR is a normalized density where a theoretical minimum is shown by an ellipsoid bead shape $\pi / 4$ which results in large voids between beads. Figure 3-8 (b) up to a fully solid part with the SR of 1.0 and no voids. The SR is calculated 
by the area of the bead divided by the potential maximum reticular area between the beads, indicated by the box around the bead. This maximum area is bounded by the width of the bead and the layer height. The width of the bead is thereby usually the diameter of the nozzle. Therefore, SR is controlled by the layer height and designated nozzle diameter. As beads are laid closer to each other the shape of the bead changes from an ellipsoid to a rectangle. The increasing density thus increases the welding area between beads which leads to higher part strength. Conclusively, it can be said that a larger welding area affects the tensile strength positively.
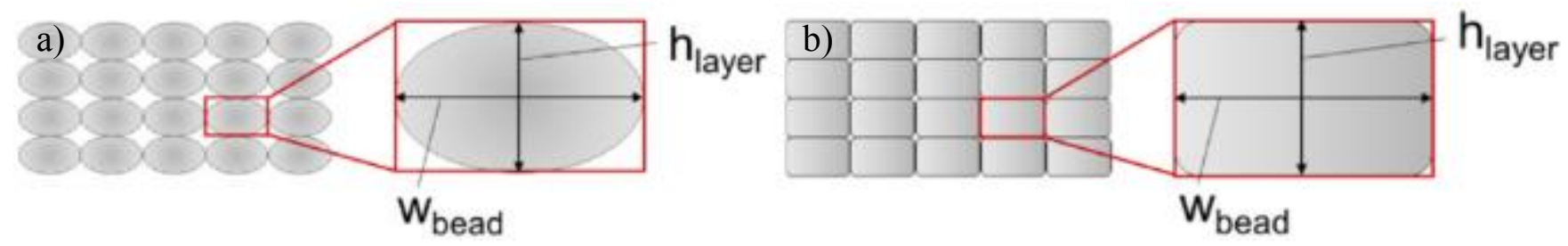

Figure 3-8 Cross-section of beads with lower (a) and higher (b) SR[112]

The detection of porosity in a composite is not straightforward. Many techniques have been employed to estimate the void content of composite parts such as ultrasonic analysis, thermography, micro-tomography, microscopy observation and acid digestion [83], [84]. Costa et al. [83] utilized an ultrasonic failure detector to characterize voids on carbon/epoxy. They used water squinters to transport the ultrasonic beams to reduce surface losses. The probes were transported by an automation system that generates a quantized C-scan record of samples. Daniel et al. [85] combined ultrasonic attenuation with image analysis to figure out a correlation between the ultrasonic attenuation and porosity. They treated values derived from optical microscope image analysis as a reference. Kite et al. [86] and Zhu et al. [87] also employed optical microscope image analysis to obtain statistical information about amount, shape, size, and orientation of voids in carbon/epoxy and glass/ epoxy systems. In their work, Kite et al. [86] showed that void content might be misestimated with more than $1 \%$ difference between image analysis and acid digestion in pre-impregnated fabrics. Further, the difference of results may increase in unidirectional laminate because of oblong, cigar-form voids. Kastner et al. [88] used X-rays micro CT scanning for the measurement of voids in composite laminates. They showed that a correct measurement could be carried out if proper threshold values are selected in the analysis. However, the threshold choice was the critical issue, and some calibrations are required for effective measurements. In addition, long scanning times and complicated volume reconstruction procedures are required to obtain high-resolution results. 
Somewhat different estimated void contents according to the measure/calculation method adopted is thus to be expected.

\subsection{Toolpath Development}

Tool path generation for 3D printers originally derived from the computer numerical control (CNC) milling problem. Current path planning algorithms for 3D printers utilize the adaptive and non-adaptive iso-planar tool path generation techniques. Various techniques for deciding layer height based on a geometric characterization of the part called adaptive slicing have been proposed [89][90]. A comprehensive review of slicing techniques was published by Pandey et al [91].

Since tool-path strategy is closely associated with the fabrication quality, most initial research about toolpaths was restricted to issues related to the manufacturing quality. Han et al.[92] proposed a deposition planning approach based on a grouping and mapping algorithm. Kao et al. [93] presented a shape optimization algorithm, which was implemented to allow high-quality spiral deposition paths. Yang et al. [94] introduced an equidistant path generation algorithm to improve the fabrication efficiency. Later, Yang [95] and Wah [96] transformed tool-path optimization in AM technology. Jin [97] proposed a mixed tool-

path generation algorithm that is the most commonly accepted in the industry. From the algorithm, zigzag tool-paths of the internal area of the layer were employed to simplify the computing processes.

\subsubsection{Methodologies of Tool-path Generation}

The tool-path required for material extrusion in AM is a predefined trajectory along which the nozzle is driven to deposit fabrication material and to form the surface layer by layer. Because the deposition quality features such as surface roughness, dimensional accuracy, and part strength are influenced by the tool-path, many efforts have been made to optimize tool-path planning. Up to now, contour-parallel-based and direction-parallel based filling strategies are mainly employed in AM. The contour-parallel tool-path comprises a series of contours, which move parallel to the boundaries of the two-dimensional crosssections [98], thus this type of fabrication accuracy is greater and more satisfactory. However, its main problem is the implementation of the offset algorithm, which is computationally expensive and complex. Figure 3-9 illustrates the difference between direction parallel and contour parallel tool-path. By contrast, direction-parallel paths contain many path segments, which correspond to back and forth motion in a fixed direction within the boundary that needs to be filled up in the interior region. This approach is 
obviously simple and fast to implement, but at the expense of fabrication precision. In order to exploit the merits of these two approaches, A recent study [97] described a fitting algorithm to establish the NURBS-based contour curve on the boundaries initially, the interior area of the model was fabricated subsequently using the direction-parallel tool-path.
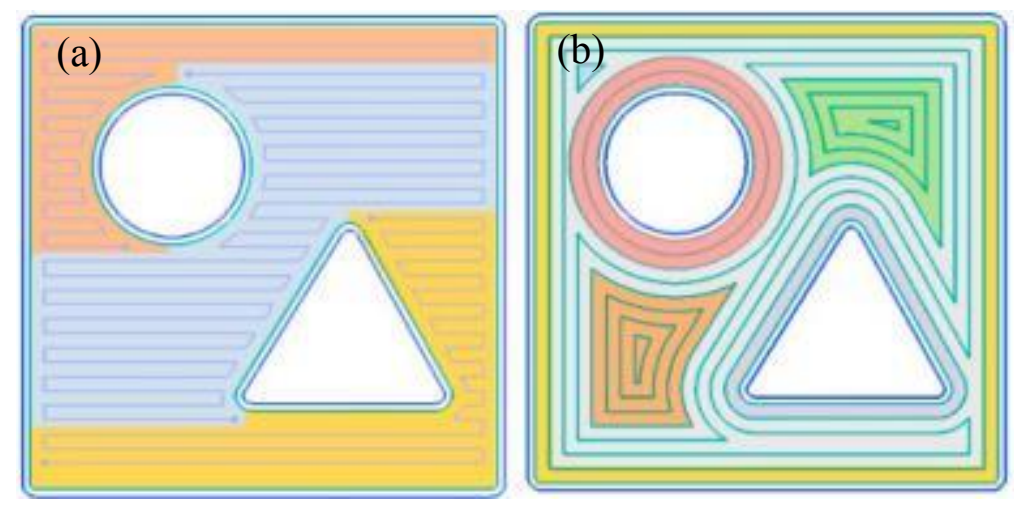

Figure 3-9 Comparison of different tool-path generation strategies; (a) Direction parallel path , (b) Contour parallel path [114]

\subsubsection{G-codes}

G-code stands for "Geometric Code". Its main function is to instruct a machine head how to move geometrically in 3 dimensions. However, it can also instruct a machine to do non-geometric things. Gcode can tell a 3D printer to extrude material at a specified extrusion rate or change its bed temperature. G-code is basically a numerical control programming language. It is easy to use and does not have advanced commands like variables, conditionals, and loops. Each line tells the printer to do a specific task. The printer executes the line one by one until it reaches the end. Normally several major g-codes govern the entire script. In this section, G0, G1 and G2 will be reviewed for fundamental study.

\subsubsection{Principle of G0 command}

Figure 3-10 shows the principle of G0 command. It tells the print head to move at maximum travel speed from the current position to the coordinates specified by the command. The head will move in a coordinate system, the nozzle will not extrude any material. This command is usually used to bring the nozzle rapidly to some desired coordinates at the start of the print or during the print. 


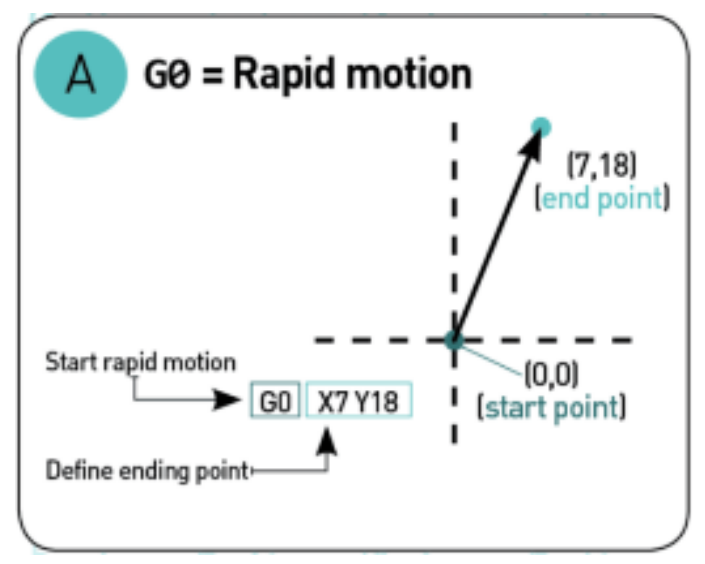

Figure 3-10 Principle of G0 command [115]

\subsubsection{Principle of G1 command}

Figure 3-11 shows the principle of G1 command. G1 tells the print head to move at specified speed. The speed is specified by the Feed rate parameter F. The printer can extrude material while executing this command at an extrusion rate specified by the Extrusion rate parameter E. More than $90 \%$ of $\mathrm{G}$ codes is composed of G1.

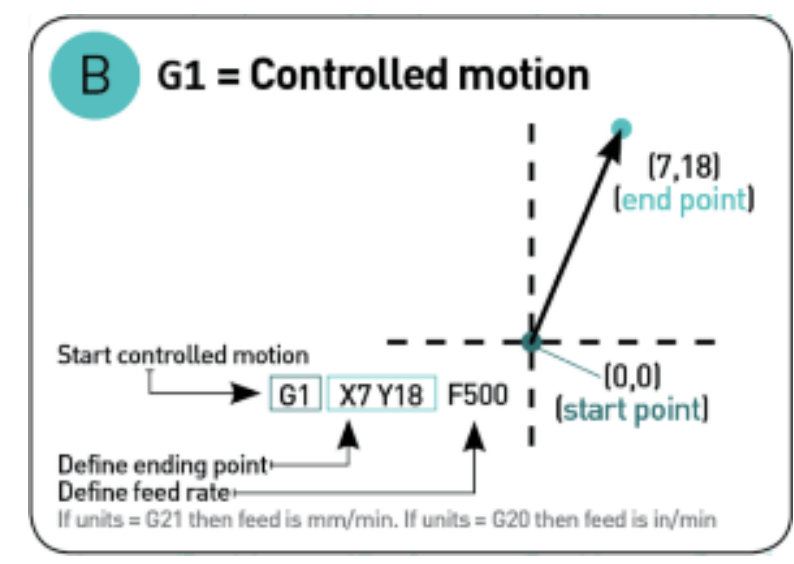

Figure 3-11 Principle of G1 command [115]

\subsubsection{Principle of G2 command}

Lastly, G2 tells the machine to move clockwise starting from its current location. The endpoint is specified 
by the coordinates $\mathrm{X}$ and $\mathrm{Y}$. The center of rotation is specified by the parameter I, which denotes the $\mathrm{X}$ offset of the current position from the center of rotation. $J$ denotes the $\mathrm{Y}$ offset of the current position from the center of rotation. Figure 3-10 illustrate the principle of G2 command.

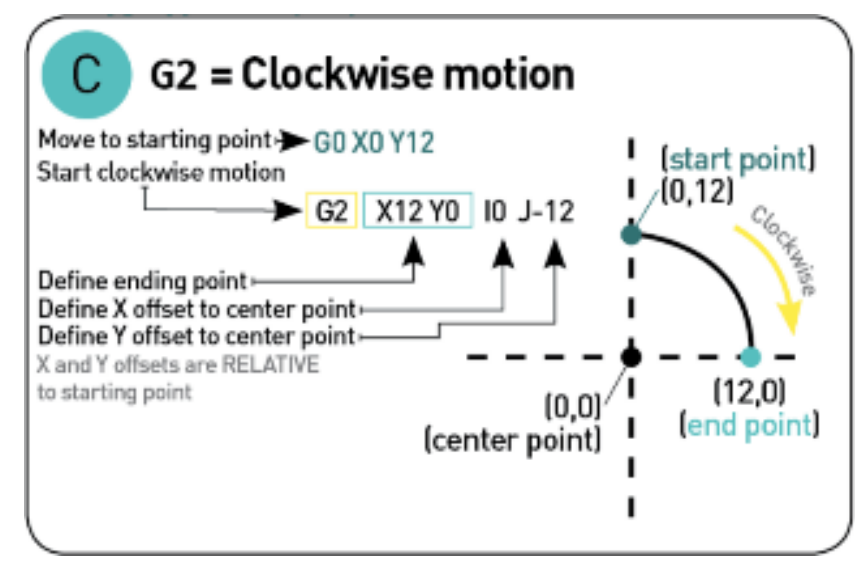

Figure 3-12 Principle of G2 command [115]

\subsection{Post-Processing for FFF printed parts}

One of the best ways to increase the strength and stiffness of FFF printed objects is by annealing them.

Annealing is an ancient process, originally used in metallurgy to increase the strength of metal objects. Annealing is one of several "heat treatments" that are used to change the physical properties of metal without changing the metal's existing shape. In essence, annealing increases the desirable characteristics of a given metal. The fundamentals of the annealing process have been adapted by for use with plastics to also increase their strength after an object has been formed.[99] Primarily an industrial plastics technique used as a finishing process, annealing can also be used by access to a kitchen oven to harden 3D prints. Figure 3-13 shows typical annealing process.
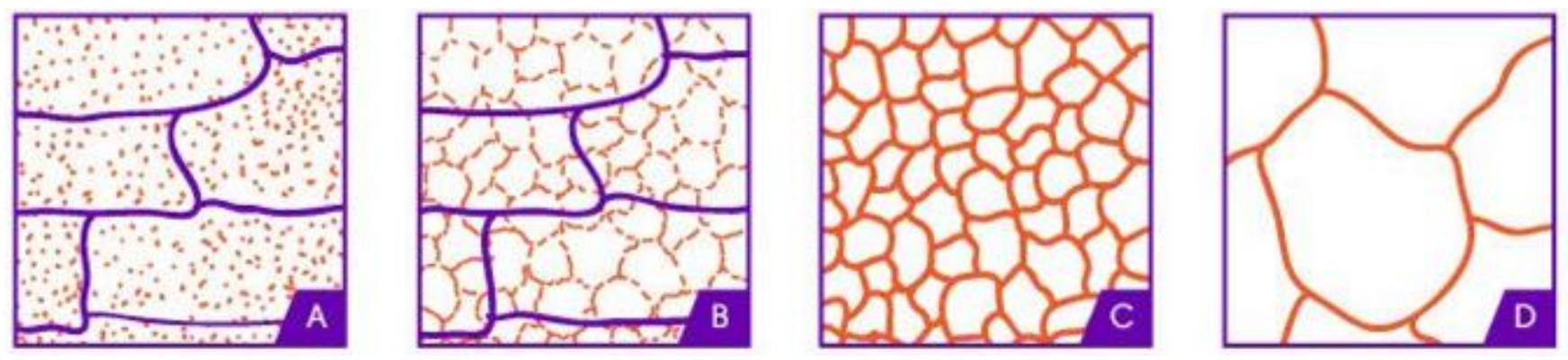

Figure 3-13 Annealing process. A) initial cold state, B) Heating: high stress areas dissipate, C) Recrystallization forms, D) Recrystallization forms[116] 
With plastics, the process is essentially the same with metal annealing. FDM printing necessarily involves heating the print material so that it can be extruded. Once extruded, the material then cools to form the printed object. Plastic is a fairly poor conductor of heat. This means that heated plastic tends to cool unevenly. This uneven cooling introduces stress into a printed object. Most thermoplastics used in FDM printing are polymers. A polymer consists of two or more substances. Each substance is made up of long molecular chains. Heating the plastic, extruding and cooling it reorganizes this structure into a more organized crystalline form. These crystals tend to be large, broadly similar to those that exist in metal after initial heating and cooling. The large crystalline-like structure of the plastic makes it prone to failure along the lines between each crystal. Also, uneven cooling due to poor heat conduction results in the polymer shrinking in different ways. This, in turn, causes different tensile forces and compression forces building up in the polymer structure. Annealing plastic involves gently reheating the substance to at its glass transition temperature or just above, but below its melting temperature, and then slowly allowing it to cool, this reheating and cooling increases the amount of crystalline structures in the plastic.

Nylon 12 is stronger and less brittle than either PLA or ABS. Its melting temperature is lower than ABS and is comparable with PLA. Like PLA, it has a low glass transition temperature which makes it easier to anneal. In addition, annealing can significantly increase its heat deflection temperature. When it combines this with its high strength, annealed Nylon 12 makes a great choice for applications where heat and durability are issues. The glass transition temperature of Nylon 12 is a surprising 41C (105F). However, it's melting temperature is a respectable $178 \mathrm{C}-181 \mathrm{C}$ (352F to $358 \mathrm{~F})$. Because of this, oven temperature to $130 \mathrm{C}-140 \mathrm{C}$ ( $266 \mathrm{~F}$ to $284 \mathrm{~F}$ ) is high enough to allow the material to soften which will release the stress caused by extrusion while increasing crystallization. As is always the case when annealing plastics, it is also low enough so that the material will not melt, flow or significantly deform. 


\section{Chapter 4 DESIGN METHODOLOGY}

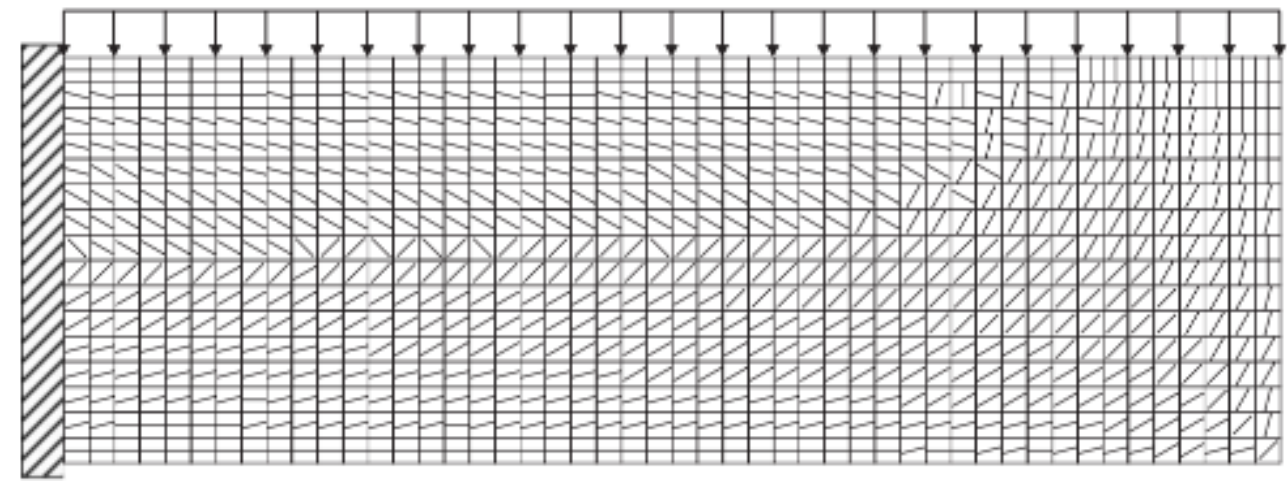

Figure 4-1 Principal directions of elements

To extrude fibers aligned with principal directions, FEA stress field was computed. Figure 4-1 shows element based principal stresses and directions of a cantilever beam under uniform distributed load. Centroids of elements are connected to create printing path. Rectangular shell elements were applied to make the path simple and to cover elements with the shortest path. The size of shell element was determined based on the diameter of printing extruder. Small size of elements guaranteed more precise printing path, however if it is much smaller than diameter of extruder, printing width may invade or cover other element sections. precise printing path, however if it is much smaller than diameter of extruder, printing width may invade or cover other element sections. Figure 4-2 shows workflow for the optimized tool-path development.

\subsection{Tool-path development}

\section{Step 1 - Finite element analysis}

In order to compute principle directions of each element, FEA stress analyses were carried out. As mentioned previously, principal direction is adapted in the determination of fiber orientation. Using the stress field output from ABAQUS ${ }^{\mathrm{TM}}$, principal directions of individual elements are computed. Then, centroids of individual elements are computed using the $\mathrm{X}, \mathrm{Y}$ coordinate information on individual nodes in input file. 


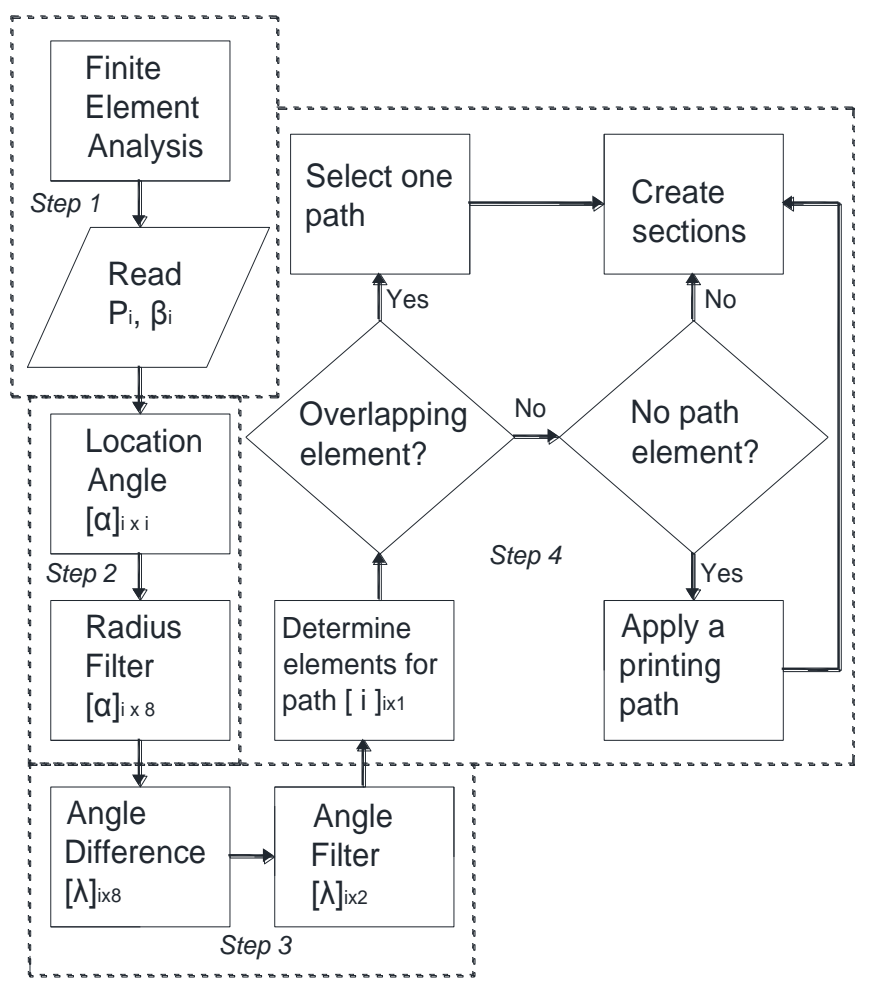

Figure 4-2 Optimized tool-path flow chart

\section{Step 2 - Radius filter and candidate elements.}

Angles of lines connecting centroids of each element are computed. This is called as location angle. $[\alpha]_{i}$ $x$ i Then, a circle with a specific radius is defined. The centroid of the starting element is defined the center of the circle. The radius is the minimum length covering centroids of elements around the starting element. Elements whose centroid is located within the circle are called as candidate elements. Figure 4-3 illustrates the concept.

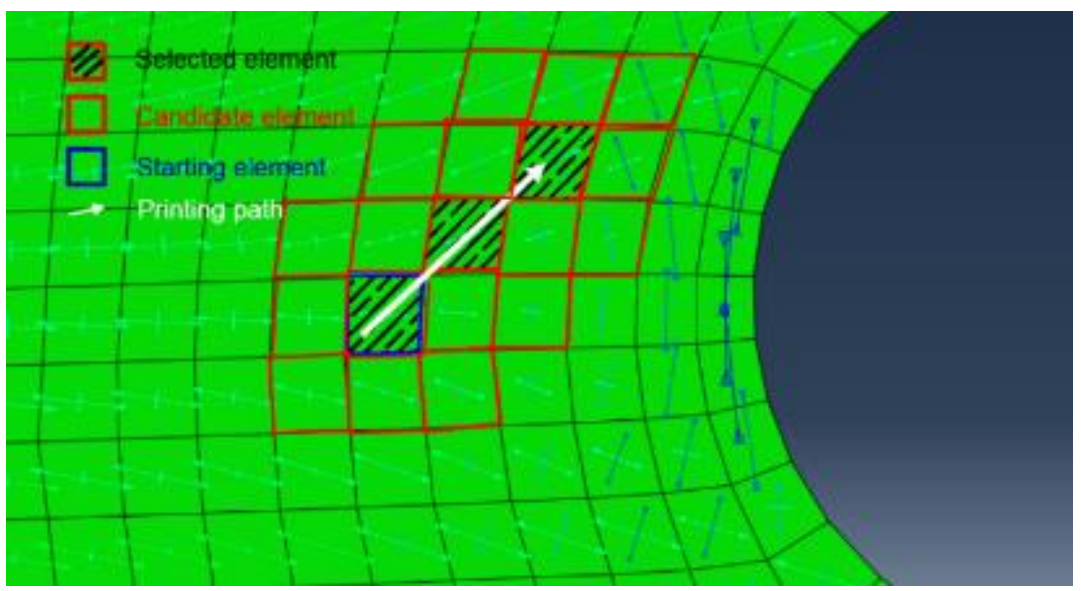

Figure 4-3 Concept of element connection 


\section{Step 3 - Angle difference filter}

Next, angle differences of location angles of candidate elements and the principal direction of the starting element are computed. Any elements whose angle difference is less than 45 degrees are selected as final candidate elements. To make paths not go back to elements already selected, cosine trigonometrical function is applied to the first, Second, and third quadrant and sine function is applied to the fourth quadrant for the location angle computation. These trigonometrical functions make location angles of some of candidate elements which already are selected large enough so that the angle difference filter screens those elements. Through this step, only two elements survive from eight candidate elements. Figure 4-4 shows the angle difference filter. Red boundary line indicates last survived two elements.

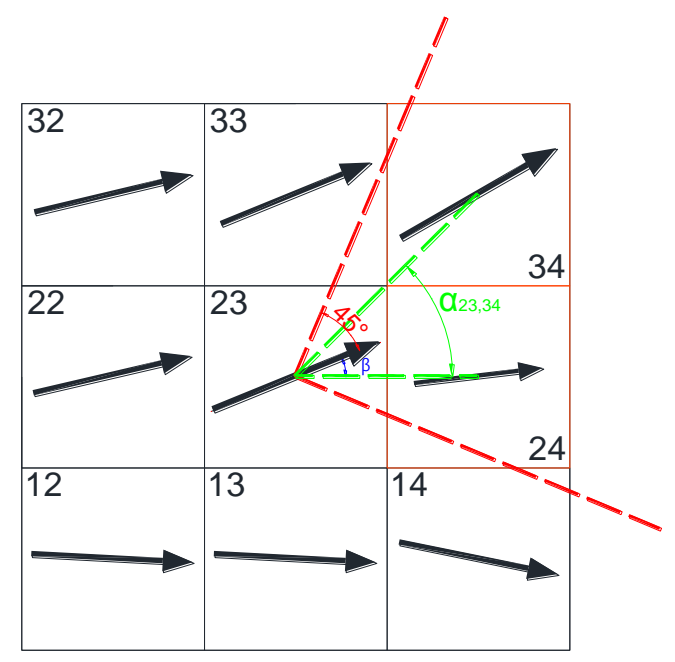

Figure 4-4 Concept of angle difference filter

\section{Step 4 - Create path and sections}

From the last two candidate elements, the element with larger principal stress is selected to connect the path. If there is no candidate element which has angle differences less than 45 degrees, the path stop connecting. A set of connecting lines creates sections. contour-parallel or direct-parallel printing method is applied based on principal directions of elements. If the path continuously goes and reaches the edge of the section, contour-parallel method is applied. otherwise, direct-parallel method is applied. In this case, the angle of infill pattern is statistically determined. To avoid overlapping path, if an element is chosen multiple times, principal stresses of previous elements on each path are checked. Then, only one

path with the previous element showing the largest principal stress is selected. Elements covered by 
identical patterns create one section. Printing path of Elements having the end of the path create boundaries. Figure 4-5 shows workflow of section and printing path generation.

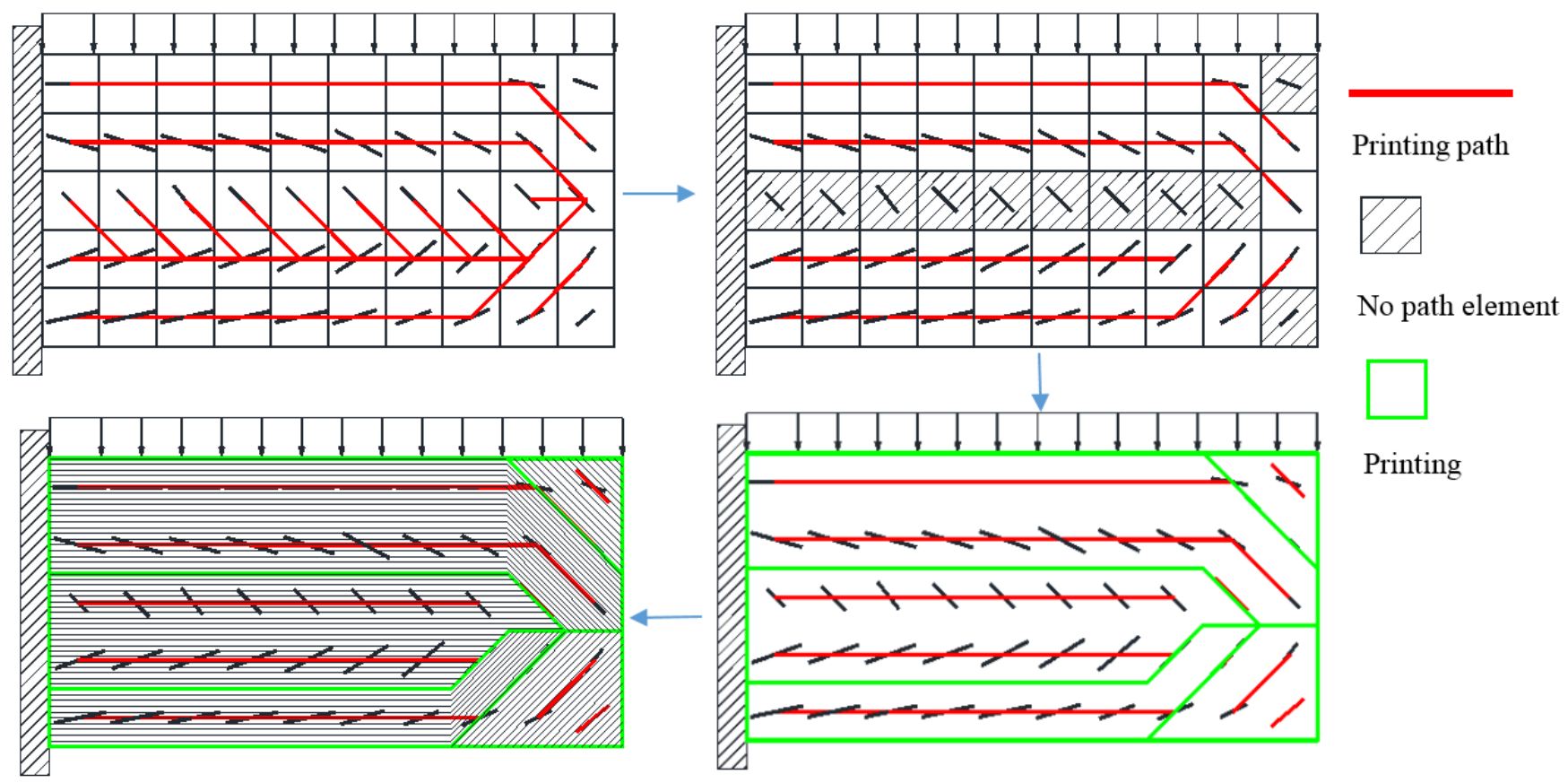

Figure 4-5 Principle of path and section development

In contrast, if an element is not chosen by any path, groups of these elements are merged into neighborhood sections. They are printed by the same pattern used in neighborhood sections. In this section consists of not chosen elements, stress level is much lower than other sections. Therefore, any printing pattern is allowed because it has a low impact on the strength of resulting objects. The other reason for this way is that the tool-path basically should focus on reducing cutting points so that extruding doesn't stop frequently to move other points which lead to reduce possible voids during the manufacturing. To guaranteed the bonding, 5\% overlapping is applied. Figure 4-6 shows the complete optimized tool-path for the plate. 


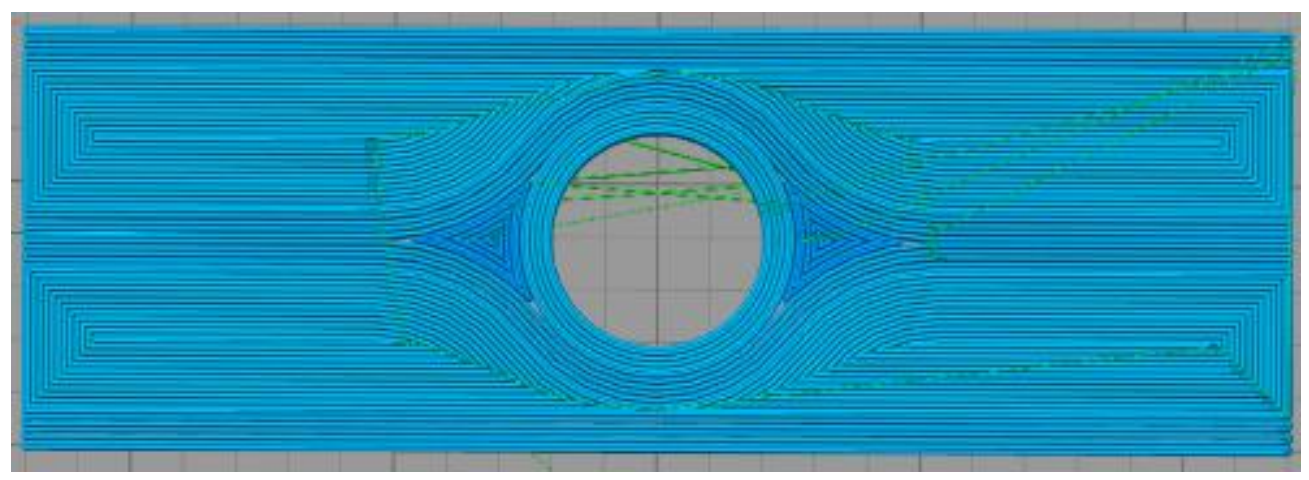

Figure 4-6 Optimized tool-path

\subsection{Finite Element Modeling}

A complete tool-path generates g-codes. Extract g-codes for outer wall printing to draw a part in ABAQUS $^{\mathrm{TM}}$. X, Y coordinate information of G1 codes is translated into a part drawing X, Y coordinates of ABAQUS ${ }^{\mathrm{TM}}$ input file. Next, C3D8 element is constructed with the uniform size. The size of the element is determined by a division parameter. If division value for $\mathrm{X}$ and $\mathrm{Y}$ axis is 50 for each, $10000=100 \times 100$ elements are created to construct a part. Small size of elements may model the part more precisely but the computation time may increase. Figure 4-7 illustrates the comparison of modeling with a different number of elements.
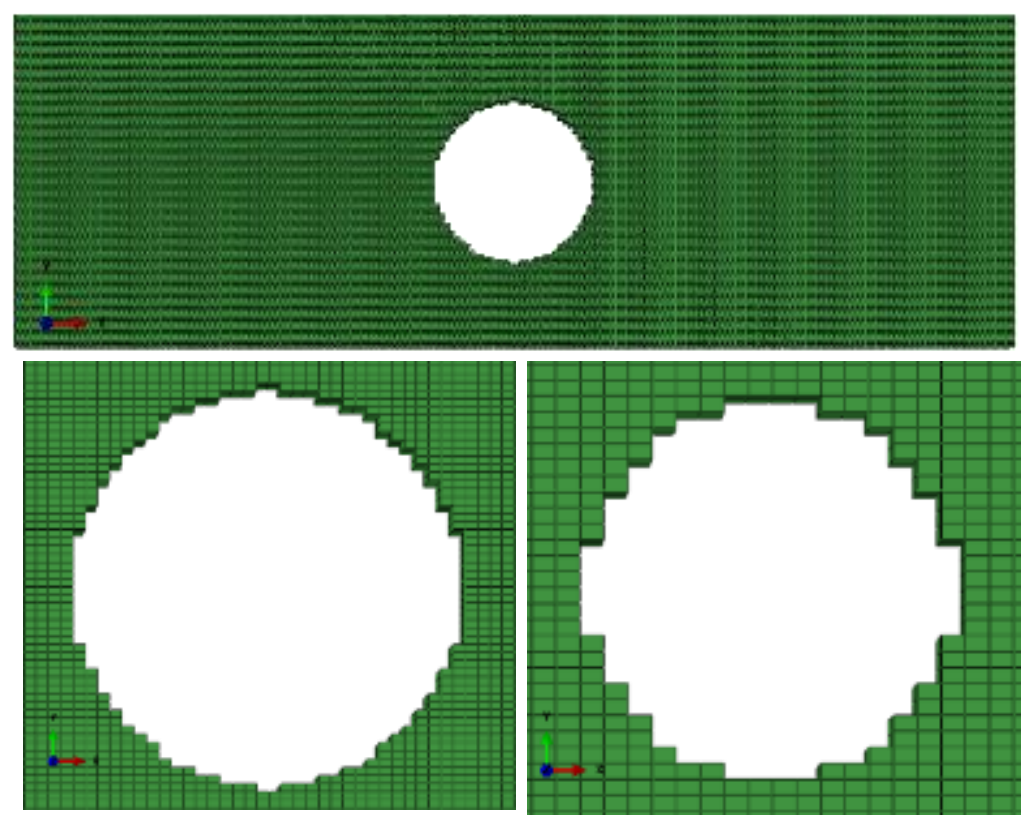

Figure 4-7 Comparison of modeling between different number of total elements; $100 * 100$ of left, $50 * 50$ of right 
Once elements are created, material orientations are applied to elements individually by using G-codes. G1 codes provide angle values between $\mathrm{X}, \mathrm{Y}$ coordinates. This angle value directly converted into material orientation. Figure 4-8 illustrates the method to determine material orientation. Lastly, orthotropic material properties from the experimental data are applied to the model. In this work, since the part size is hand-size residual stress induced during the manufacturing is ignored.

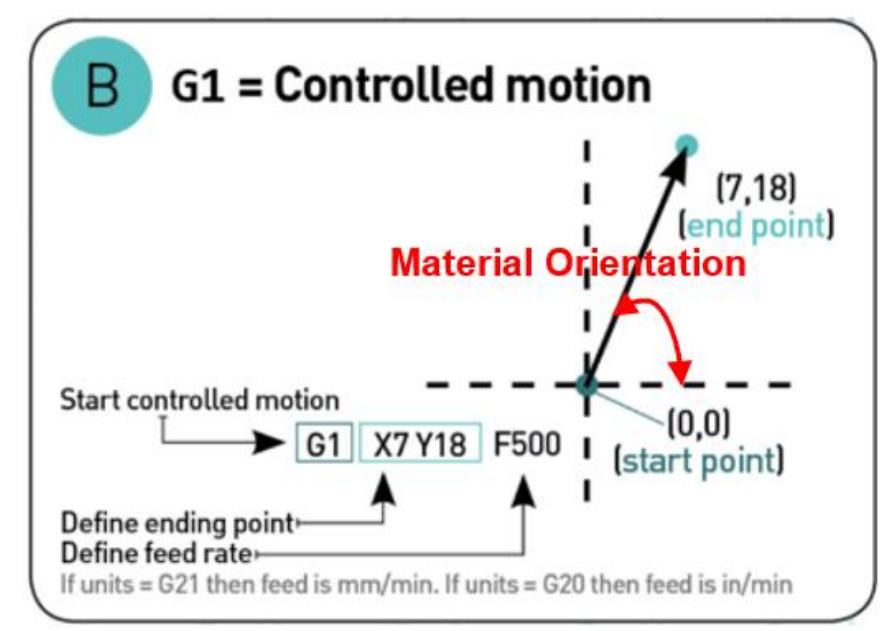

;NOZZLE_DIAMETER:0.6

G0 F7714.3 X93.849 Y93.593 Z0.4

;TYPE:WALL-INNER

G1 F1800 X94.057 Y93.669

E0.05049

G1 X94.197 Y93.72 E0.08446

G1 X94.555 Y93.866 E0.17261

G1 X95.1 Y94.124 E0.31009

G1 X95.512 Y94.355 E0.41779

G1 X95.808 Y94.545 E0.49798

Figure 4-8 Determination of material orientation 


\section{Chapter 5 MATERIAL CHARACTERIZATION}

\subsection{Tensile Test}

Specific material characteristics were measured, respective to the material plane 1-2 in Figure 5-1, using specially oriented specimens. The direction 1 (red color) is FFF line deposition and direction 2 is perpendicular to this line deposition. From the specimens oriented at $0^{\circ}$, the tensile modulus in the deposition direction $E_{1}$, the Poisson ratio $v_{12}$ and the tensile strength $S_{1}$ were determined. From the specimens oriented at $90^{\circ}$, the tensile modulus perpendicular to the deposition direction $\mathrm{E}_{2}$, the Poisson ratio $v_{21}$ and the tensile strength $S_{2}$ were determined. From the specimens oriented at $\pm 45^{\circ}$, the shear modulus at the 1-2 plane $G_{12}$ and the shear strength $S_{12}$ were determined. Three specimens per sample were tested for each one of the three orientation cases and for each printing material, totalizing 9 test runs.

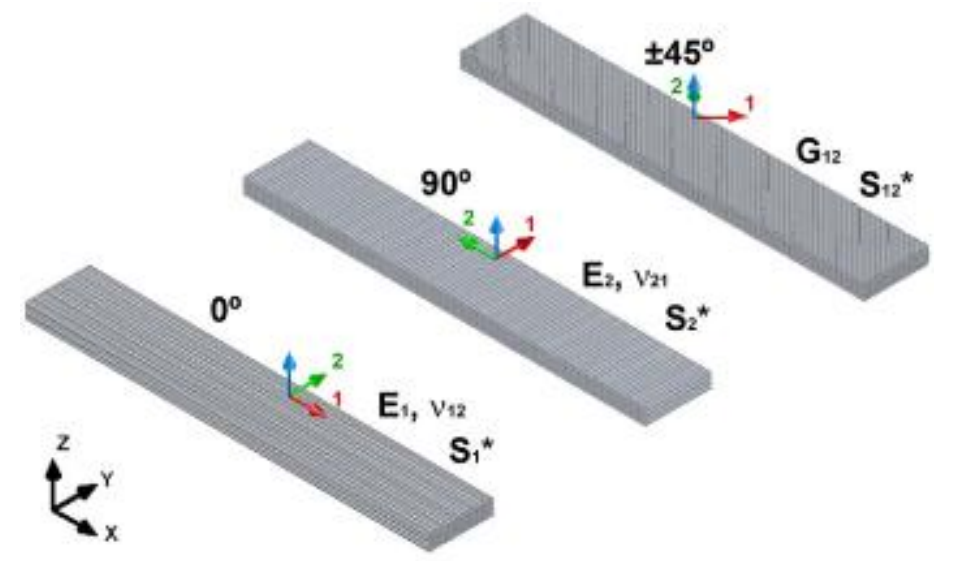

Figure 5-1 Illustration of printing orientations $\left(0^{\circ}, 90^{\circ}\right.$ and $\left.\pm 45^{\circ}\right)$

Here, standards for polymers and laminated composite materials were followed in adaptation, as the example from the literature [100]. For the determination of stiffness and strength properties at material directions 1 and 2 it was chosen the standard ASTM D638-10 [101], devoted to tensile tests of polymers. The specimen shape employed was the I-type ("dog bone"), with outer length and width respectively of $165 \mathrm{~mm}$ and $19 \mathrm{~mm}$, as shown in Figure 5-2 (a). The nominal thickness was $3.3 \mathrm{~mm}$ reached with 11 printed layers, which were deposited either at $0^{\circ}$ or $90^{\circ}$ along the tensile direction. Therefore, the 
stacking sequences for the specimens were respectively $\left[0^{\circ}\right]_{11}$ and $\left[90^{\circ}\right]_{11}$. To obtain the $1-2$ plane shear stiffness and strength properties of the printed material, it was used the standard ASTM D3518-13[102], specific for polymer matrix composite materials. The specimens employed were rectangular and had 25 $\mathrm{mm} \times 200 \mathrm{~mm}$, as illustrated in Figure 5-2(a). The nominal thickness was $4.8 \mathrm{~mm}$, reached with 16 printed layers whose stacking sequence was $\left[ \pm 45^{\circ}\right] 4 \mathrm{~s}$ (symmetric), as imposed by the followed standard. The test specimens were produced by Ultimaker 2+, with a printing envelope of $215 \mathrm{~mm} \times 210 \mathrm{~mm} \times 180 \mathrm{~mm}$, nozzle diameter of $0.4 \mathrm{~mm}$. The printing parameters employed were: nozzle extrusion temperature of 260 ${ }^{\circ} \mathrm{C}$, heat bed temperature of $110^{\circ} \mathrm{C}$, deposition line (layer) height $0.3 \mathrm{~mm}$, printing speed of $20 \mathrm{~mm} / \mathrm{sec}$. The CarbonX-Nylon was used as carbon-fiber nylon filament manufactured by 3DXtech, and is made from the 4043D resin reinforced with chopped short carbon fibers in a weight fraction of $10 \%$. The diameter of the $3 \mathrm{D}$ printer filaments employed was $2.85 \mathrm{~mm}$. The experiments were performed using an SHIMAZU AGS-X HC universal testing machine, with a load cell of capacity of $10 \mathrm{kN}$ as shown Figure 5-2(b). All the specimens were loaded up to material failure at a displacement rate of $1 \mathrm{~mm} / \mathrm{min}$. The data acquisition rate was $10 \mathrm{~Hz}$ for displacements and loads measured.
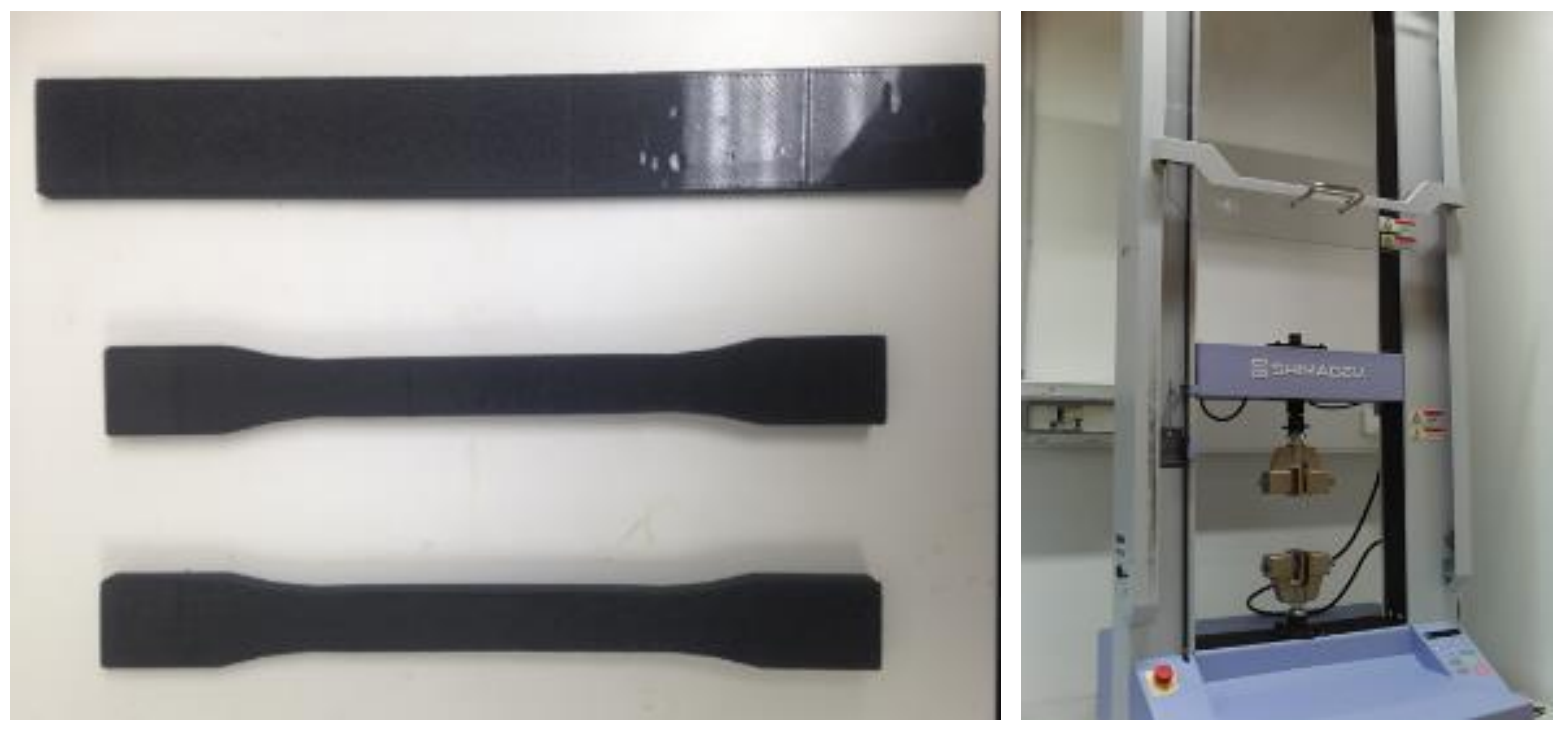

Figure 5-2 Employed specimens (a): tensile (“dog bone”, lower) and shear (rectangular, upper) and SHIMAZU tensile machine (b) 

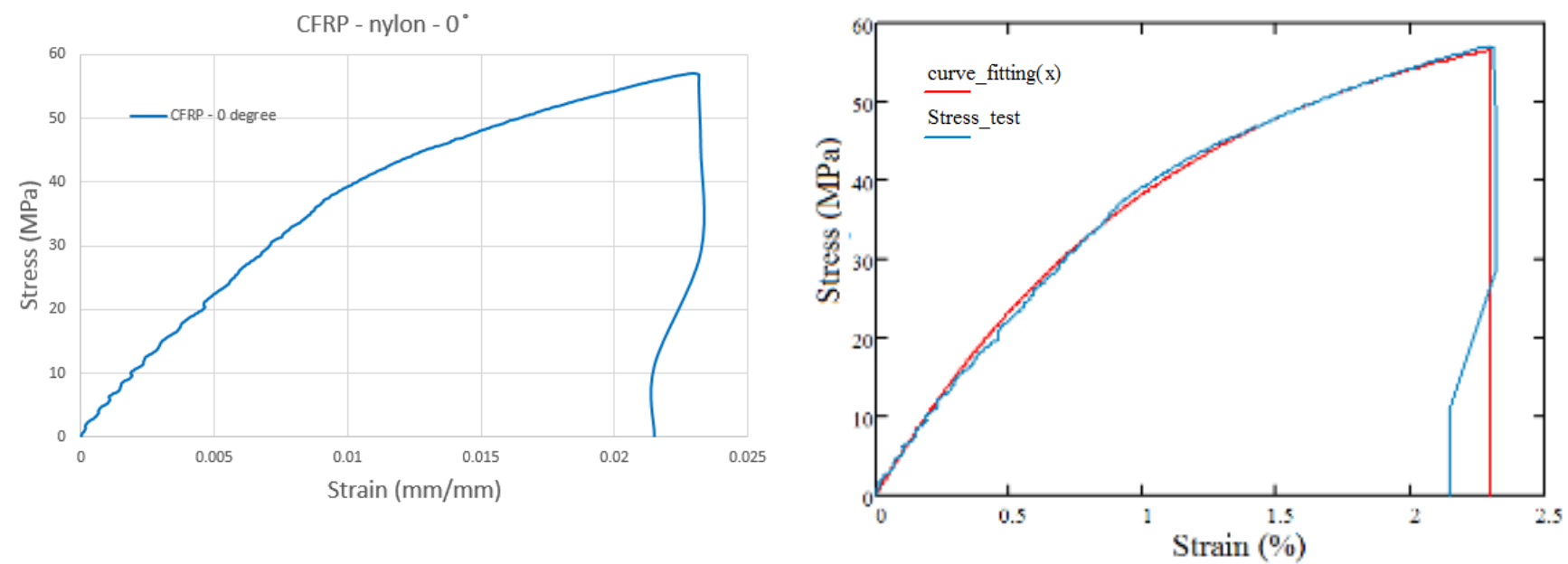

Figure 5-3 raw data (a) and curve fitting (b) of strain stress curve of CFRP-nylon printed at $0^{\circ}$ direction

The material properties required for FEM simulation were extracted from the stress-strain curves. To find the trend in stress-strain curve more clearly, curve fitting was computed as shown Figure 5-3. Figure 5-4 shows the stress-strain curves for the three identical specimens printed along directions $0^{\circ}, 90^{\circ}$, respectively. The deviation in the elastic moduli and yield strength values are less than $3 \%$. Thus, the material properties are consistent within a given orientation. However, when the parts are printed in different directions significant differences are observed in the material properties. The tensile properties were calculated in accordance to the standard ASTM D638-10 [101]. The specimens were loaded at the direction $\mathrm{x}$, perpendicular to $\mathrm{y}$, according to Figure 5-1. Tensile moduli $\mathrm{E}_{i}\left(\mathrm{E}_{1} ; \mathrm{E}_{2}\right)$ and Poisson's ratios $v_{i j}\left(v_{12} ; v_{21}\right)$ were calculated by:

$$
E_{i}=\frac{\sigma_{x}^{0.25}-\sigma_{x}^{0.05}}{\varepsilon_{x}^{0.25}-\varepsilon_{x}^{0.05}}, \quad \nu_{i j}=\frac{\varepsilon_{x}^{0.25}-\varepsilon_{x}^{0.05}}{\varepsilon_{y}^{0.25}-\varepsilon_{y}^{0.05}} .
$$

In Eq 5-1, $\varepsilon_{x}^{k}$ are longitudinal normal strains of value $\mathrm{k} \%$ (close to $0.05 \%$ and $0.25 \%$ in the case), $\varepsilon_{y}^{k}$ are transverse normal strains at the level of $\varepsilon_{x}^{k}$ and $\sigma_{x}^{k}$ are tensile stresses corresponding to $\varepsilon_{x}^{k}$. Therefore, both $\mathrm{E}_{i}$ and $v_{i j}$ are given by chordal values obtained from the data gathered. The tensile stresses $\sigma_{x}^{k}$ are given by:

$$
\sigma_{x}^{k}=\frac{P^{k}}{A}
$$

In Eq 5-2 $\mathrm{P}_{k}$ is the tensile load at $\varepsilon_{x}^{k}$ and $\mathrm{A}$ is the cross sectional area of a specimen. Finally, considering 
that $\mathrm{P}_{\max }$ is the maximum load applied to a specimen, the tensile strengths $S_{i}^{*}\left(S_{1}^{*}, S_{2}^{*}\right)$ were calculated by:

$$
S_{i}^{*}=\frac{P_{\max }}{A}
$$
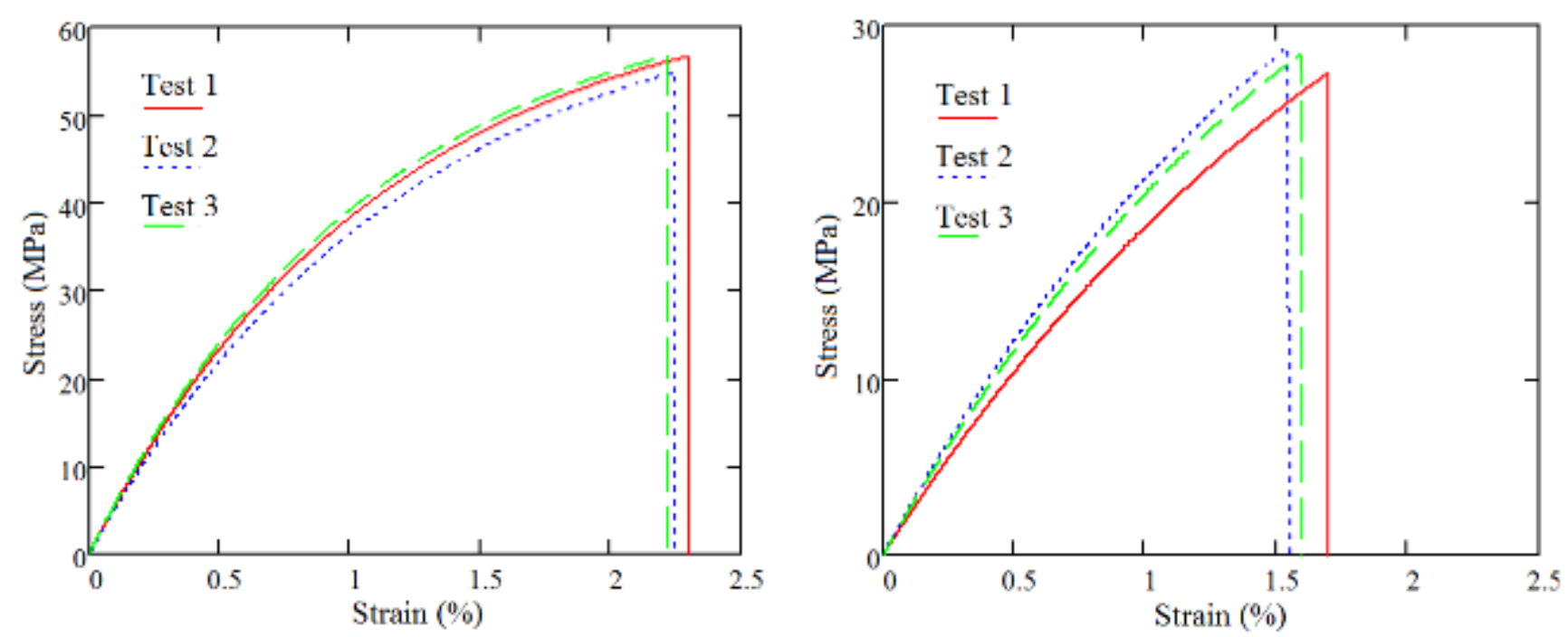

Figure 5-4 Stress vs Strain data for CarbonX-Nylon printed at $0^{\circ}$ and $90^{\circ}$

Figure 5-5 show the stress-strain curves of the specimen printed along the direction $\pm 45^{\circ}$. The shear properties were calculated in view of the standard ASTM D3518-13. Again, the specimens were loaded at the direction $\mathrm{x}$, the applied load $\mathrm{P}$ was measured and strains $\varepsilon_{x}$ and $\varepsilon_{y}$ were computed by using displacement data. The shear modulus $G_{12}$ was calculated by:

$$
G_{12}=\frac{\tau_{12}^{0.6}-\tau_{12}^{0.2}}{\gamma_{12}^{0.6}-\gamma_{12}^{0.2}}
$$

In Eq 5-4, $\gamma_{12}^{l}$ are shear strains of value $l \%$ (close to $0.2 \%$ and $0.6 \%$ in the case) and $\gamma_{12}^{l}$ are shear stresses corresponding to $\gamma_{12}^{l}$. Therefore, G12 is obtained as a chordal modulus. The shear strains $\gamma_{12}^{l}$ are calculated using:

$$
\gamma_{12}^{l}=\varepsilon_{x}^{l}-\varepsilon_{y}^{l}
$$

Eq 5-5 regards the measured strains in the outer layers of the shear specimens (oriented at $45^{\circ}$ ) and implied coordinate transformation relations. The $\varepsilon_{x}^{l}$ and $\varepsilon_{y}^{l}$ are the measured normal strains (longitudinal and 
transverse, respectively) when $\gamma_{12}^{l}$ happens. Based on transformation relations for stresses, the shear stresses $\tau_{12}^{l}$ are given by:

$$
\tau_{12}^{l}=\frac{P^{l}}{2 A}
$$

In Eq 5-6, A is the cross-sectional area of the specimen and Pl is the applied load when $\gamma_{12}^{l}$ happens. Finally, the shear strength $S_{12}^{*}$ was calculated using:

$$
S_{12}^{*}=\frac{P_{\max }^{s}}{2 A}
$$

In Eq 5-7, $P_{\max }^{s}$ is the maximum load at or below $\gamma_{12}^{5}$ (shear strain equal to 5\%), as ASTM D3518-13 recommends.

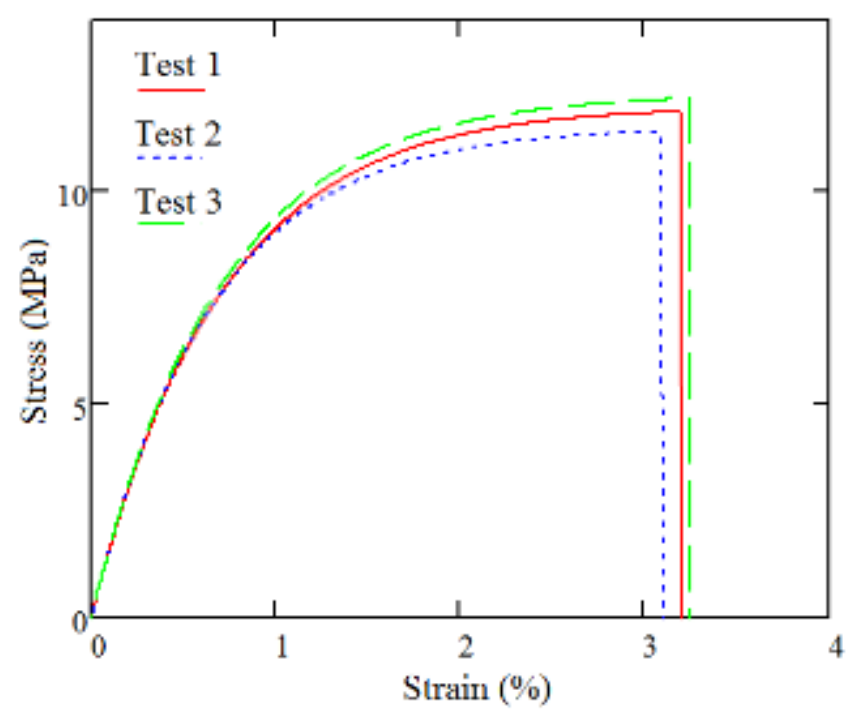

Figure 5-5 Stress vs Strain data for CarbonX-Nylon printed at $\pm 45^{\circ}$

With the same experimental procedure above, tensile tests of Nylon 12 were performed as shown in Figure 5-6. Figure 5-7 show the stress-strain curves for specimens printed along directions $0^{\circ}, 45^{\circ}$, and $90^{\circ}$, respectively. The material properties are consistent within a given orientation. However, when the parts are printed in different directions, significant differences are observed in the material properties. The deviation in the elastic moduli and yield strength values are less than 5\%. Detailed test results show up in Appendix. It shows no difference between stiffness of samples. However, directional dependency of failure strength was revealed. Samples printed at $0^{\circ}$ direction shows $30 \%$ and $87 \%$ higher failure strength 
than samples printed at $45^{\circ}$ and $90^{\circ}$ directions. This is because FFF built parts behave like laminated materials, made by orthotropic layers whose principal direction of highest stiffness is the printing orientation direction. This can be assumed due to some facts. At first, specimens were 3D printed only using rectilinear infill with volume fraction of $100 \%$, which means that fused material lines were deposited only parallel to each other, forming layers which are placed on top of each other to build a specimen. Second, the 3D printing parameters of deposition line height and width were kept the same in all specimens. Therefore, it can be considered as the experiments were all made upon a material of constant microstructure, placed at specific orientations, following the FFF deposition.
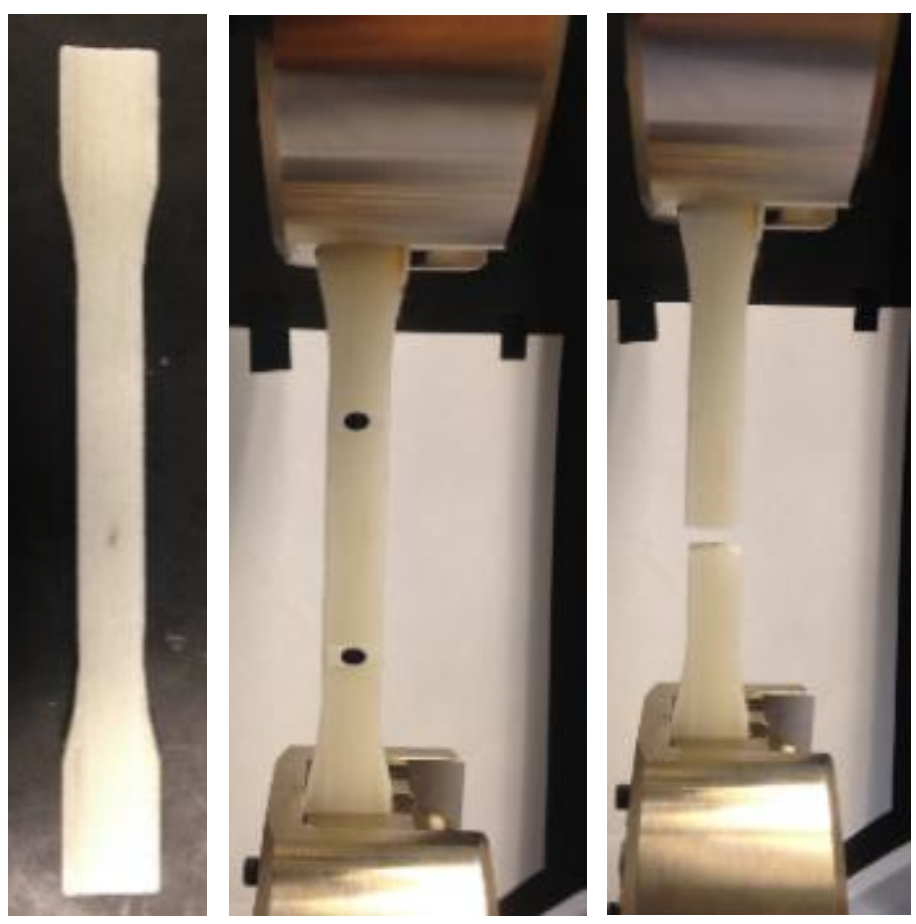

Figure 5-6 Tensile tests of Nylon 12. 


\section{Tensile test result}

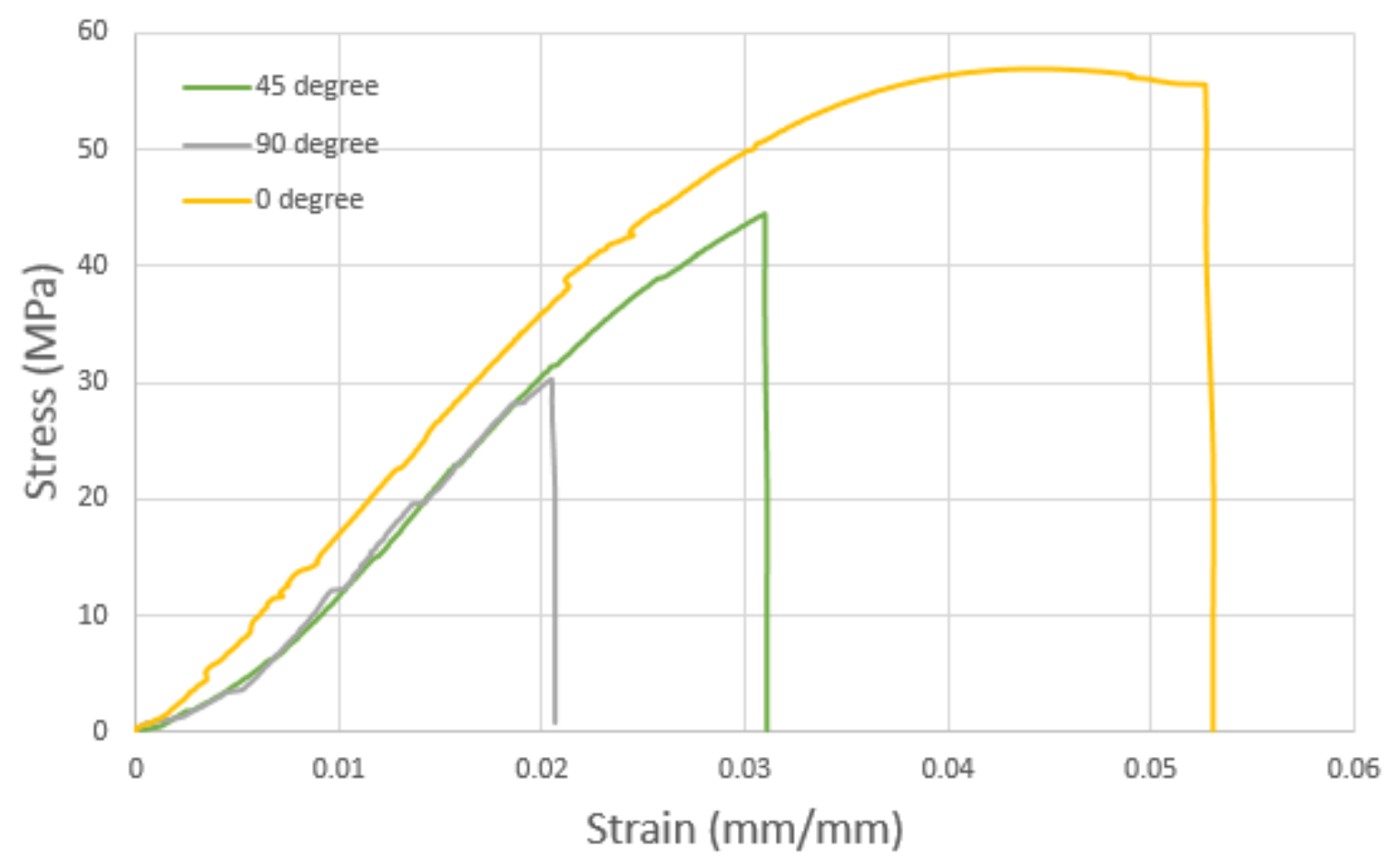

Figure 5-7 Tensile test result of Nylon 12 samples printed at various angles

Table 5-1 shows the comparison of mechanical properties from test results and book value of CarbonXNylon and Nylon 12. Young modulus from experimental results shows approximately $94 \%$ of theoretical value. Theoretical values of CarbonX-Nylon and Nylon 12 are shown in Appendix. Poisson coefficients were a lot different for the CarbonX-Nylon, with $v_{12}$ about 2.0 times higher than $v_{21}$. This can be explained by the differences found for $E_{1}$ and $E_{2}$ for CarbonX-Nylon, which shows that it is much more difficult to stretch the material at the direction 1 (printing direction) than at the direction 2, a typical behavior found in polymer composites reinforced with unidirectional carbon fibers. From the results in Table 5-1, it can be noticed that the material strength $S_{1}^{*}$ for the pure isotropic Nylon 12 and $S_{1}^{*}$ for the CarbonX-Nylon were about the same, indicating that the short carbon fibers did not change substantially those properties. However, the CarbonX-Nylon had lower strains at failure as can be seen from Figure $5-4$, indicating a more brittle behavior than that seen in the Nylon 12. It can be noted that the short carbon fibers in CarbonX-Nylon favored the known naturally brittle behavior of Nylon 12. 
Table 5-1 Comparison of mechanical properties between isotropic pure Nylon 12 and CarbonX-Nylon

\begin{tabular}{|c|c|c|c|c|c|c|}
\hline \multirow{2}{*}{ Property } & \multirow{2}{*}{ Direction } & \multicolumn{2}{|c|}{ CFRP-nylon } & \multicolumn{2}{|c|}{ Nylon 12} & \multirow{2}{*}{ ASTM } \\
\hline & & Experimental & Theoretical & Experimental & Theoretical & \\
\hline Young's & $0^{\circ}$ & & \multirow{2}{*}{$4.39 \mathrm{GPa}$} & $1.73 \mathrm{GPa}$ & \multirow{2}{*}{$1.70 \mathrm{GPa}$} & \multirow{2}{*}{ D638 } \\
\hline Modulus & $90^{\circ}$ & $2.15 \mathrm{GPa}$ & & $1.61 \mathrm{GPa}$ & & \\
\hline Shear Modulus & $\pm 45^{\circ}$ & $1.12 \mathrm{GPa}$ & - & $0.591 \mathrm{GPa}$ & - & D3518 \\
\hline \multirow{2}{*}{$\begin{array}{l}\text { Tensile } \\
\text { Strength }\end{array}$} & $0^{\circ}$ & $56.6 \mathrm{MPa}$ & \multirow{2}{*}{$63.9 \mathrm{MPa}$} & $54.1 \mathrm{MPa}$ & \multirow{2}{*}{$48 \mathrm{MPa}$} & \multirow{2}{*}{ D638 } \\
\hline & $90^{\circ}$ & $28.3 \mathrm{MPa}$ & & 27.MPa & & \\
\hline Shear Strength & $\pm 45^{\circ}$ & $11.9 \mathrm{MPa}$ & - & $10.5 \mathrm{MPa}$ & - & D3518 \\
\hline \multirow{2}{*}{ Elongation } & $0^{\circ}$ & $2.30 \%$ & \multirow{2}{*}{$4.00 \%$} & $5.30 \%$ & \multirow{2}{*}{$15.00 \%$} & \multirow{2}{*}{ D638 } \\
\hline & $90^{\circ}$ & $1.59 \%$ & & $2.10 \%$ & & \\
\hline \multirow{2}{*}{ Poisson's ratio } & $v 12$ & 0.391 & - & 0.341 & - & \multirow{2}{*}{ D638 } \\
\hline & $v 21$ & 0.203 & - & 0.331 & - & \\
\hline
\end{tabular}

\subsection{Creep Test}
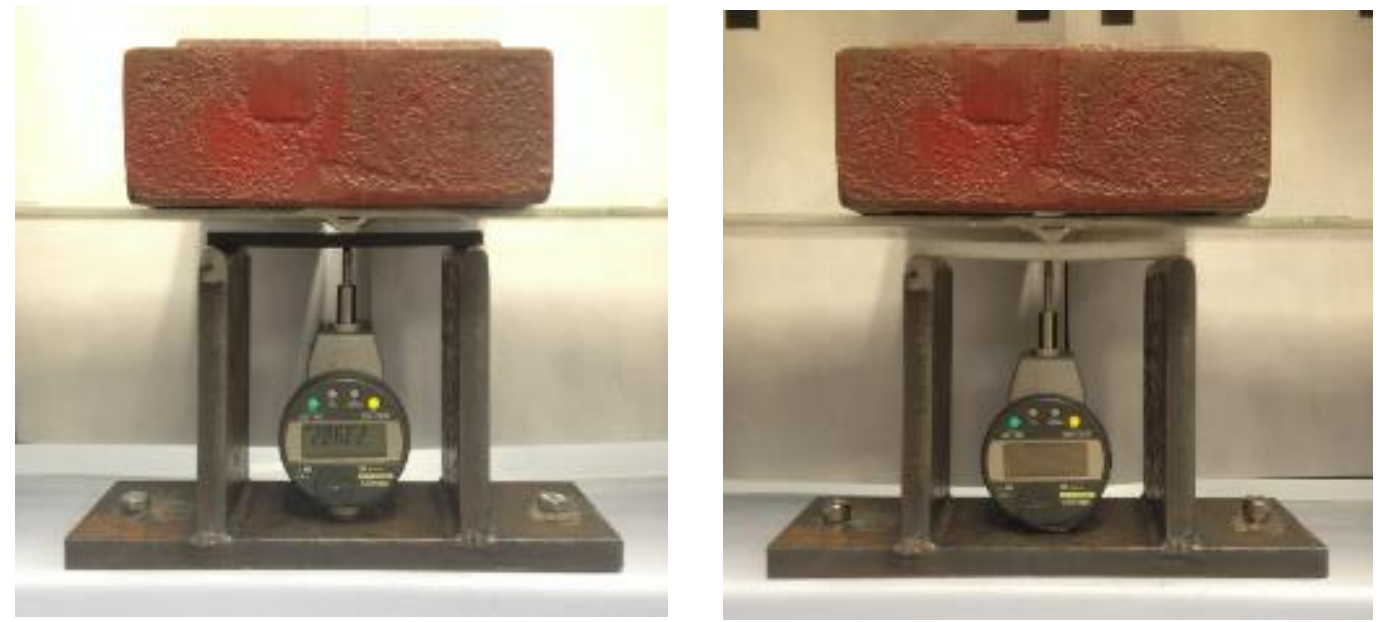

Figure 5-8 Creep test method for CFRP and Nylon 12

Flexural creep behavior of CFRP and Nylon 12 was investigated. Test method was followed by ASTM D2990 [9]. Rectangular plates with dimensions $120 \mathrm{~mm} \mathrm{X} 12 \mathrm{~mm} \mathrm{X} \mathrm{20mm} \mathrm{were} \mathrm{used.} 0^{\circ}, 45^{\circ}$, and $90^{\circ}$ of fiber orientations were applied to manufacturing for comparison. Also, nylon 12 specimens printed at $0^{\circ}$ direction were used to see the material advantage of CFRP. To set up the test platform, two 20 lbf steel 
blocks were prepared to apply dead load and a digital deflectometer was installed on the bottom to measure the displacement as shown in Figure 5-8.

Displacement at the middle of specimen was measured with the following approximate time schedule: 1 , 6,12 , and $30 \mathrm{~min} ; 1,2,5,20,50$, and 100 hours. The maximum fiber stress for each specimen was calculated as follows:

$$
\mathrm{S}=\frac{3 P L}{2 b d^{2}}
$$

Where: $S=$ Stress, $P=$ load, $L=$ span, $b=$ width, $d=$ depth.

The maximum strain at the mid-span was calculated as follows:

$$
\mathrm{r}=\frac{6 D d}{L^{2}}
$$

Where: $\mathrm{r}=$ maximum strain, $\mathrm{D}=$ maximum deflection at mid-span
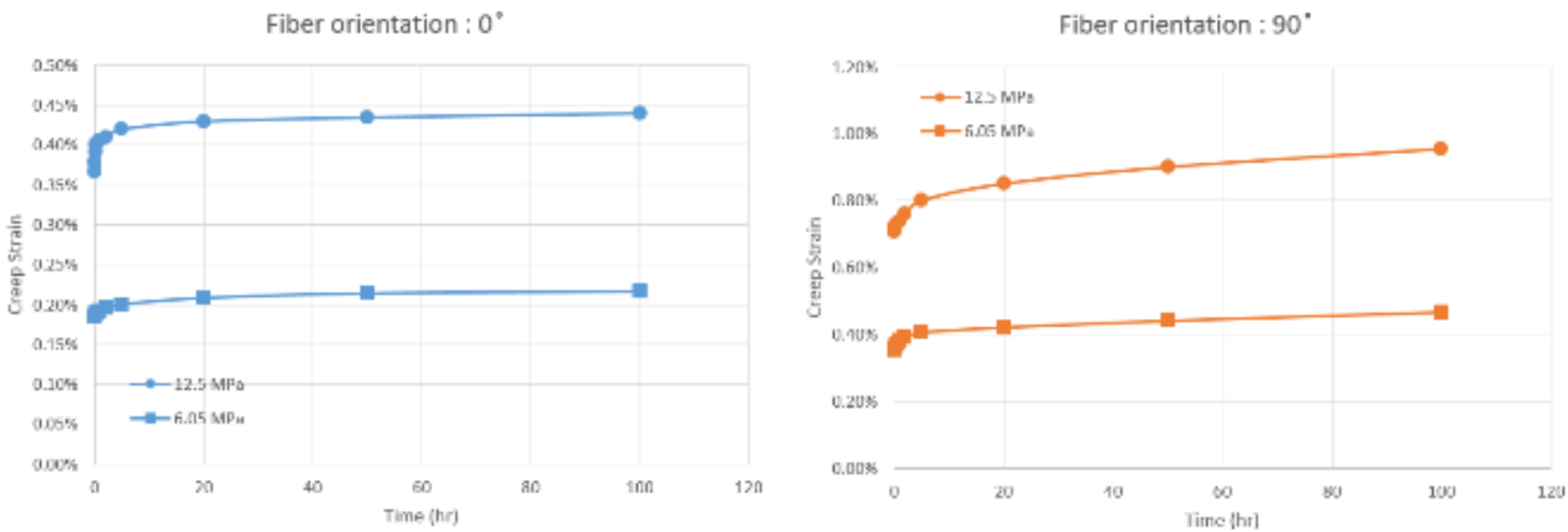

Fiber orientation : $45^{\circ}$

Nylon 12
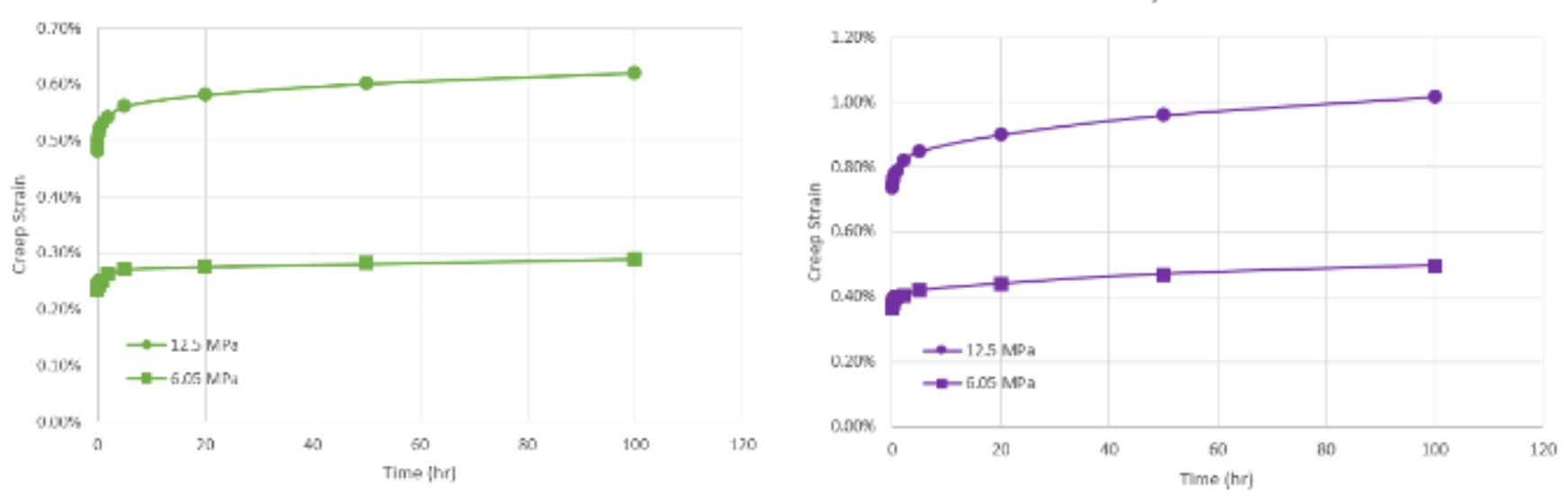

Figure 5-9 Creep test results of CFRP and Nylon 12with different fiber orientations 
The experimental data from the creep tests were plotted to analyze the flexural creep response of CFRP and Nylon 12. Figure 5-9 shows the experimental creep curves for CFRP with $0^{\circ}, 45^{\circ}$, and $90^{\circ}$ fiber orientation and Nylon 12 test specimens under two different stress levels, respectively. It is found that a significant creep strain was noticed, especially at higher stress levels. In general, the creep strain increases as the angle of the fiber direction with the loading axis increases. Because the elastic modulus of the $0^{\circ}$ specimen is higher than that of the $45^{\circ}$ specimen, the creep strain of the $0^{\circ}$ specimen is less than that of the $45^{\circ}$ specimen. Figure 5-10 shows the comparison of creep response Nylon 12 and CFRP with three different fiber orientations. It is noted that CFRP with $90^{\circ}$ fiber orientation shows slightly lower creep strain than those of Nylon 12. This is because fibers of the specimen don't fully contribute to the strength.

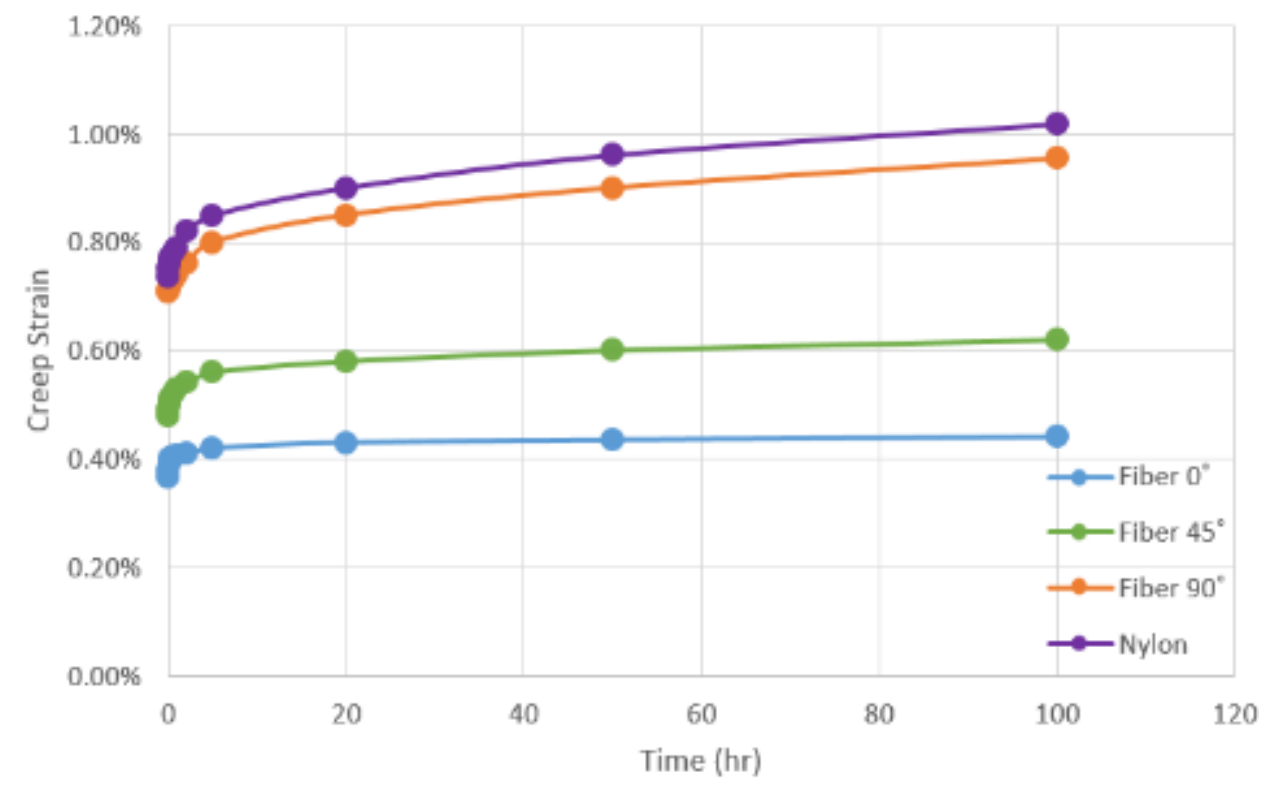

Figure 5-10 Comparison of creep response for CFRP and Nylon 12 


\subsection{Fiber orientation distribution of CFRP}

The fiber orientation created during 3D printing determines the properties of CFRP. Figure 5-11 shows an optical microscope image of CFRP with cylindrical fiber that appears on the section as an ellipse. In this research, the method used by Bay and Tucker [61] was followed to characterize the fiber orientation of samples. For manual digitization, Matlab ${ }^{\mathrm{TM}}$ was used to process images. It digitizes four endpoints of the major and minor axes as shown in Figure 5-12 (a).
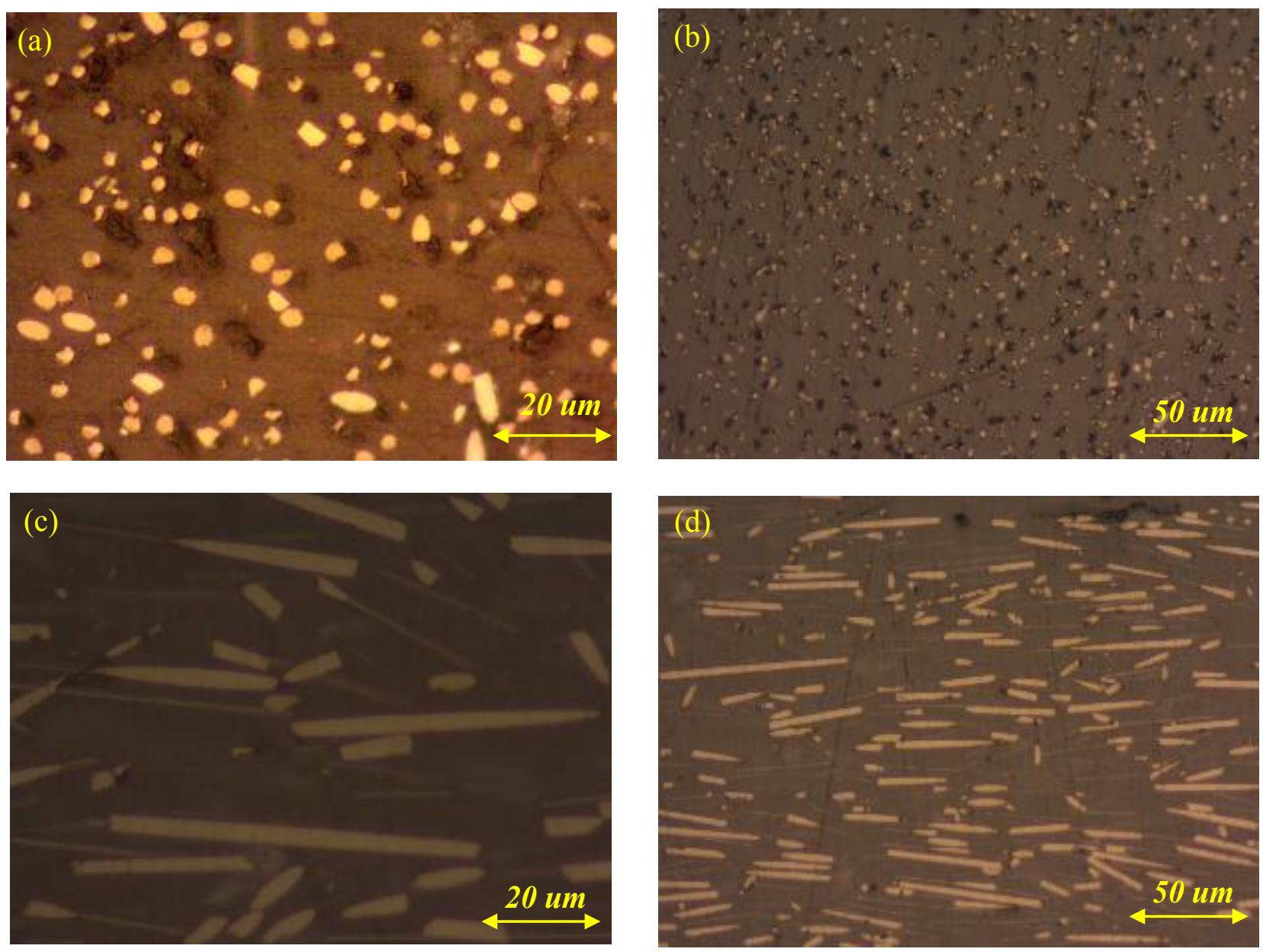

Figure 5-11 Sample micrographs of a polished specimen obtained from optical microscope

a) Cross section at $0^{\circ}$ (printing direction), magnitude 50x; b) cross-section at $0^{\circ}$, magnitude 20x; c) cross section at $90^{\circ}$, magnitude $50 \mathrm{x}$; d) cross-section at $90^{\circ}$, magnitude $20 \mathrm{x}$ 

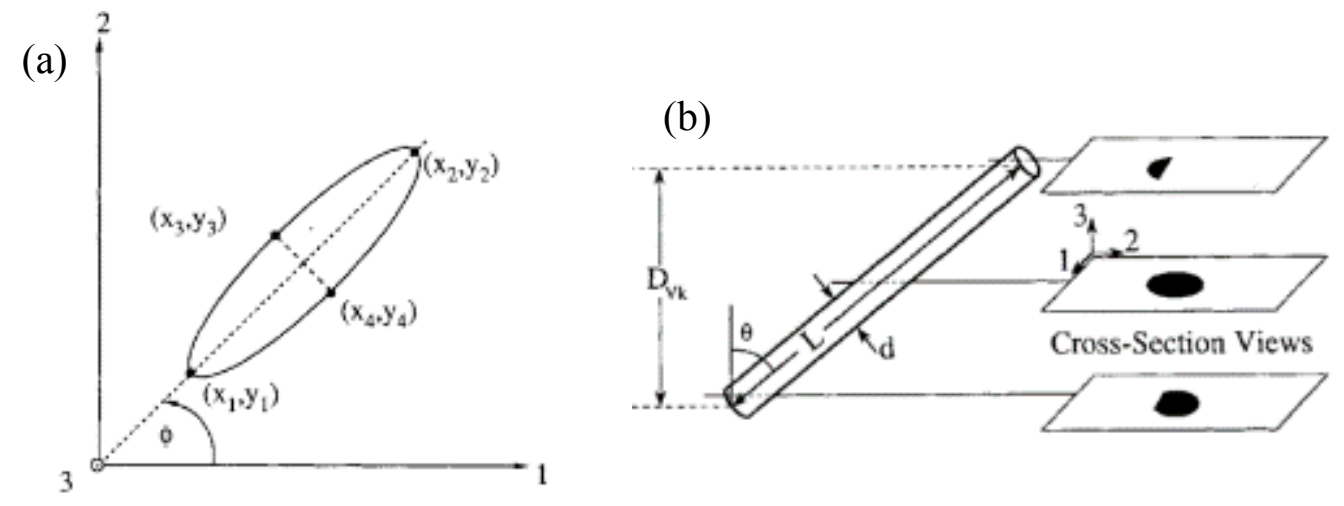

Figure 5-12 (a) Elliptical cross section of cylindrical fiber showing definition of axes' endpoints and inplane angle $\Phi$, (b) Cross sections of a fiber inclined 0 from the 3-axis. [70]

Assuming the length and diameter of fibers are uniform as approximately 100um and 5um, the measurement was processed finding only the ends of the major axis. The image was broken into pixels; each pixel has a value corresponding to the intensity of light at its location on $\mathrm{X}, \mathrm{Y}$ Cartesian coordinate system. These digital images are first subjected to a thresholding operation, making each pixel either black or white as shown in Figure 5-13.
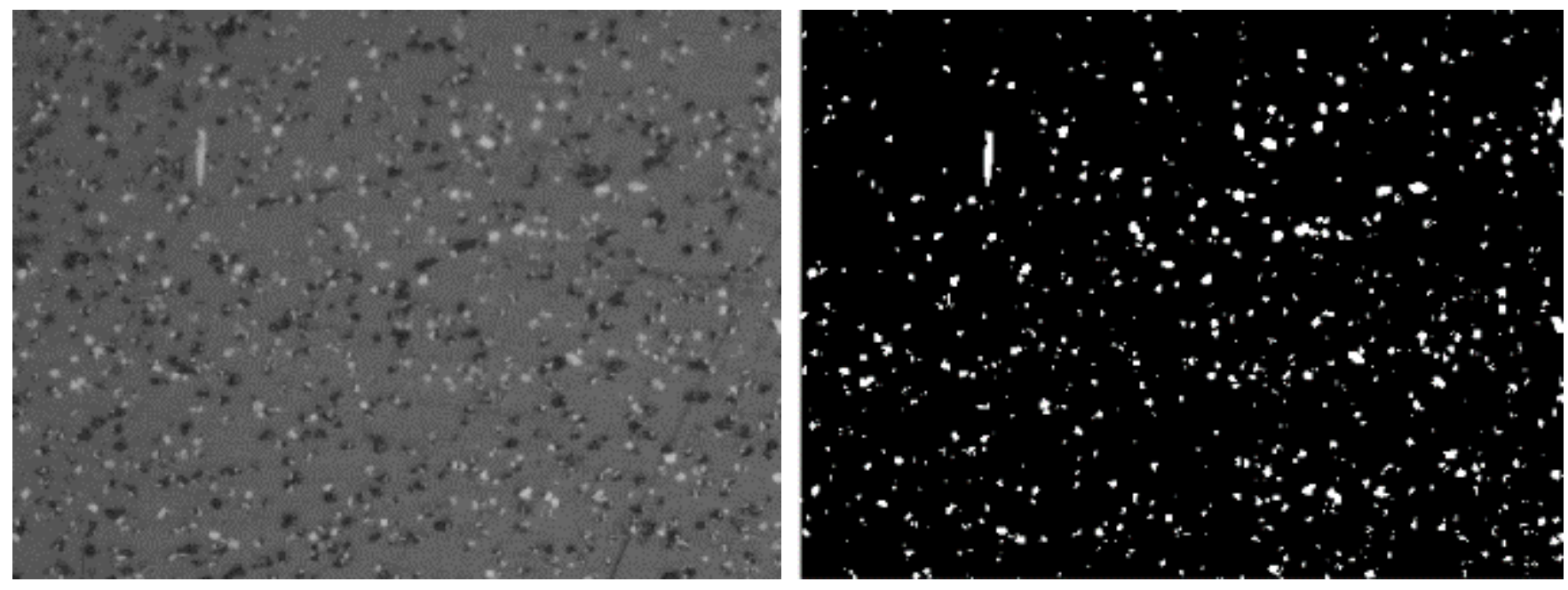

Figure 5-13 fiber capture converting pixels into black and white.

The next step is to identify the group of pixels representing each fiber and determines the relevant dimensions. It measured the chord length in several directions and then take the maximum and minimum values as the major and minor axes, respectively. Using the cross-sectional area and major/minor 
diameters, check if each cross section is roughly elliptical. If those fibers whose cross sections are not elliptical, they would be broken fibers. Those fibers will be filtered as shown in Figure 5-14.
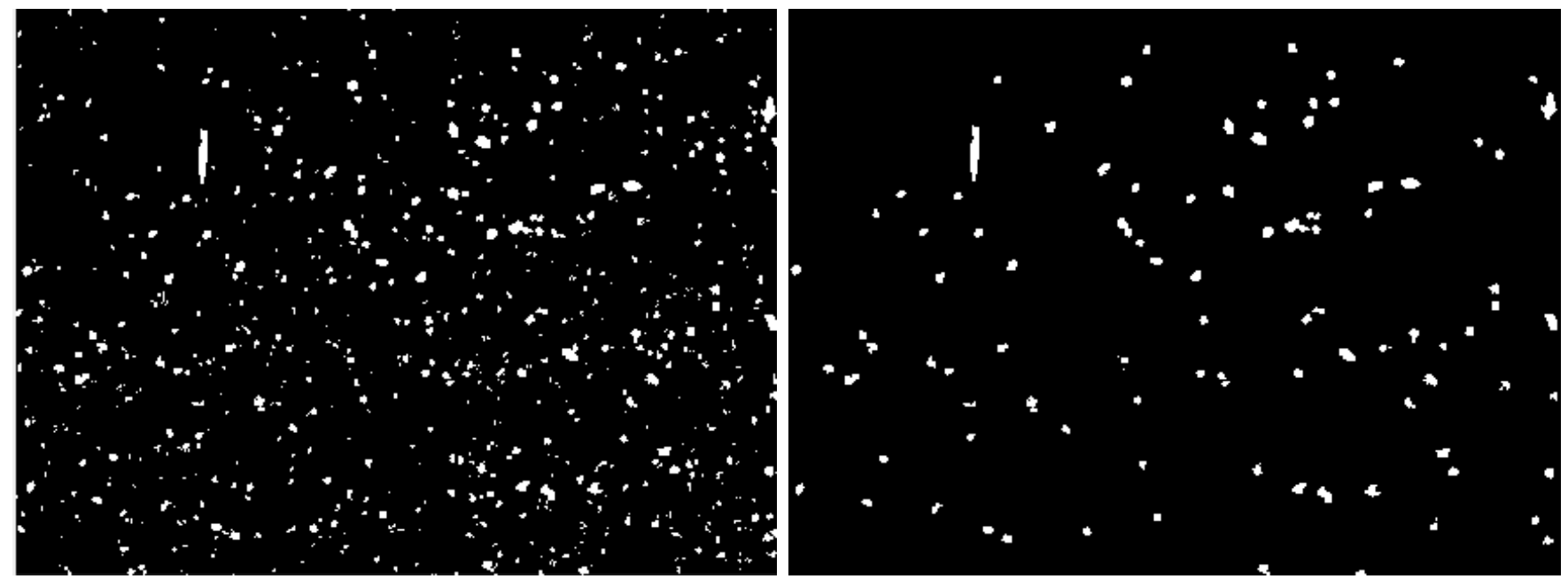

Figure 5-14 Process of broken fibers filtering

The third step is to determine the components of $p$ for each fiber as shown in Figure 5-15. The spatial orientation of a single rigid, axisymmetric fiber is easy to describe.

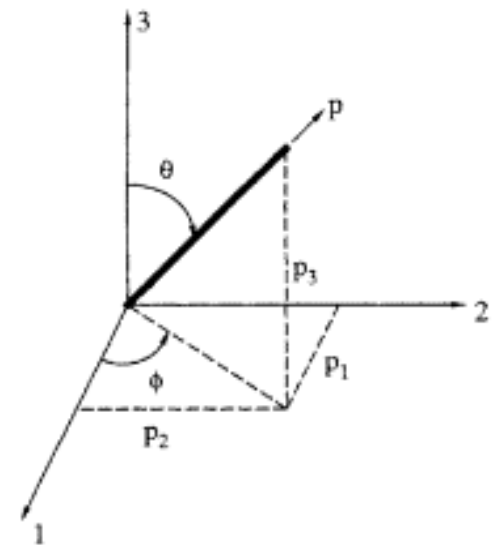

Figure 5-15 Definition of Eulerian angles showing components of unit vector p.[70]

Angles $\theta$ and $\Phi$ are used to define its orientation. Alternately, a unit vector $\mathrm{p}$ can be used; the Cartesian components of $\mathrm{p}$ are 


$$
\begin{aligned}
& \mathrm{p}_{1}=\sin \theta \cdot \cos \phi \\
& \mathrm{p}_{2}=\sin \theta \cdot \sin \phi \\
& \mathrm{p}_{3}=\cos \theta
\end{aligned}
$$

Unless stated otherwise, the image section was taken as 1-2 plane, and the 3-axis to be normal to the plane. If the data are collected as the coordinates of the four endpoints, the minor axis $\mathrm{m}$ is the diameter of the fiber:

$$
\mathrm{m}=\sqrt{\left(\mathrm{x}_{3}-\mathrm{x}_{4}\right)^{2}+\left(\mathrm{y}_{3}-\mathrm{y}_{4}\right)^{2}}
$$

$\mathrm{X}$ and $\mathrm{Y}$ were defined as

$$
\mathrm{X}=\mathrm{x}_{2}-\mathrm{x}_{1} \quad \mathrm{Y}=\mathrm{y}_{2}-\mathrm{y}_{1}
$$

Then the major axis $\mathrm{M}$ is given by

$$
M=\sqrt{X^{2}+Y^{2}}
$$

From the geometry of Figure 5-12, the relevant functions of $\theta$ and $\Phi$ are

$$
\sin \phi=\frac{Y}{M} \quad \cos \phi=\frac{X}{M} \quad \cos \theta=\frac{m}{M} \quad \sin \theta=\sqrt{1-\frac{m^{2}}{M^{2}}}
$$

The value of $p_{i}$ and $p_{j}$ is denoted for the nth fiber as $\left(a_{i j}\right)_{n}$. The values of $\left(a_{i j}\right)_{n}$, in terms of major and minor axis, anthe d distance of $\mathrm{X}$ and $\mathrm{Y}$ axis expressed as $\mathrm{M}, \mathrm{m}, \mathrm{X}$, and $\mathrm{Y}$ show up in Table 5-2. $\mathrm{m}$, $\mathrm{X}$, and $\mathrm{Y}$ are treated as independent variables, but $\mathrm{M}$ is a function of $\mathrm{X}$ and $\mathrm{Y}$. Since other scalar measures of orientation such as the Hermans orientation function are defined in terms of trigonometric functions of $\Theta$ and $\phi$, they can also be expressed as functions of $\mathrm{m}, \mathrm{M}, \mathrm{X}$, and Y. Table 5-2 shows components of second-order orientation tensor for CFRP fibers. $a_{11}$ is dominant orientation tensor for CFRP.

Table 5-2 Second-order orientation tensor values for CFRP fibers

\begin{tabular}{ccc}
\hline Orientation & Equation & $\%$ \\
\hline a11 & $\sin ^{2} \theta \cos ^{2} \phi$ & 0.825 \\
a12 & $\sin ^{2} \Theta \cos \phi \sin \phi$ & 0.123
\end{tabular}




\begin{tabular}{ccc} 
a13 & $\sin \theta \cos \theta \cos \phi$ & 0.082 \\
$\mathrm{a} 22$ & $\sin ^{2} \theta \sin ^{2} \phi$ & 0.03 \\
$\mathrm{a} 23$ & $\sin \theta \cos \theta \sin \phi$ & 0.004 \\
$\mathrm{a} 33$ & $\cos ^{2} \phi$ & 0.0056 \\
\hline
\end{tabular}

The full Matlab codes to perform this analysis are attached in Appendix. Histograms of fiber orientation tensor $a_{11}, a_{12}$, and $a_{13}$ were established as shown in Figure 5-16, Figure 5-17, and Figure 5-18, respectively. 40 number of microscope images analyzed and 10,000 number of fiber orientations were computed. To see how many fibers are aligned with printing direction, $a_{11}, a_{12}$, and $a_{13}$ of tensors were computed. Once histograms were established, average tensor values were computed. those values are $0.82,0.12$, and 0.08 for $a_{11}, a_{12}$, and $a_{13}$, respectively. Table 5-2 shows the orientation tensor matrix of fibers.

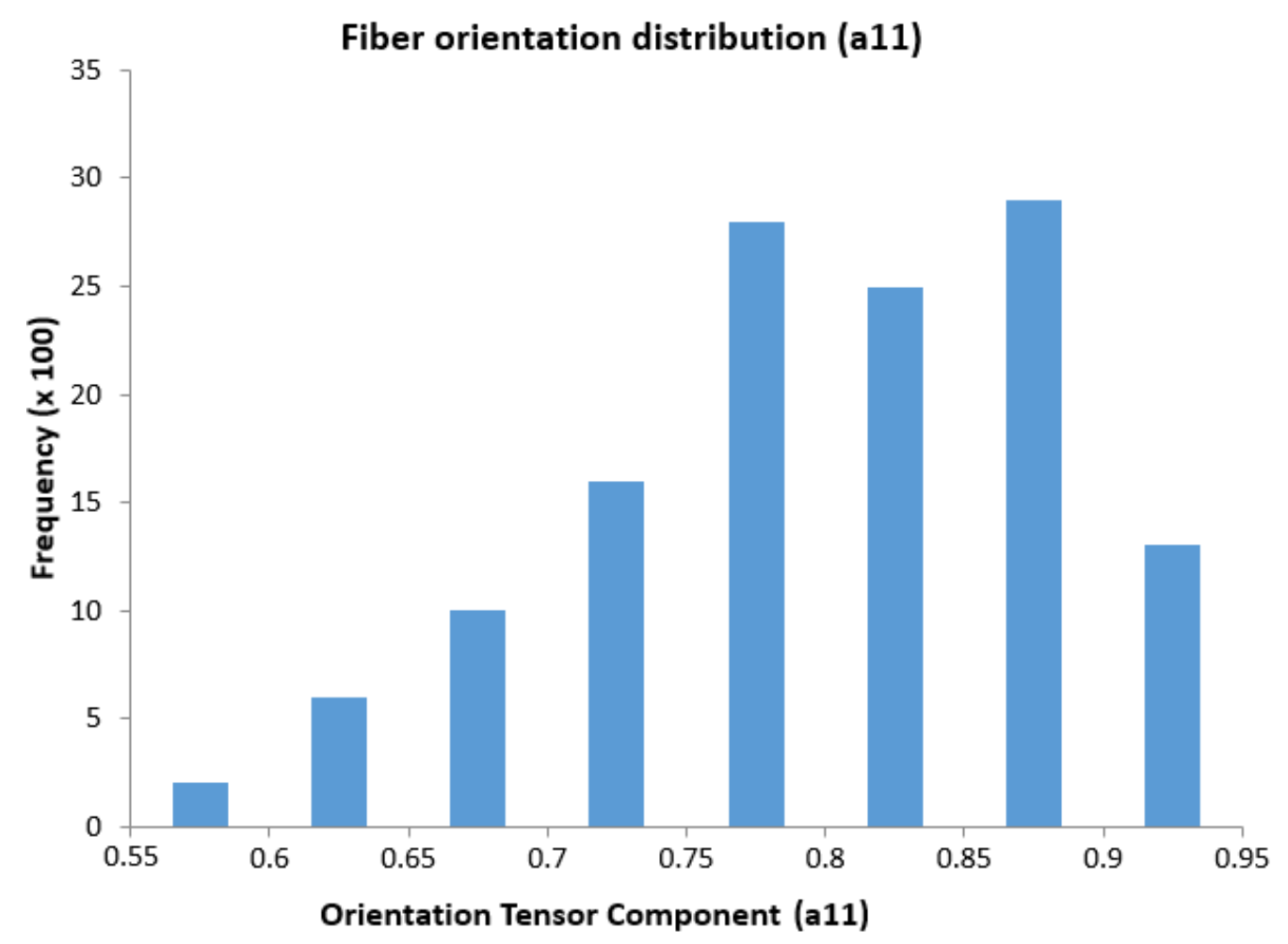

Figure 5-16 Fiber orientation distribution of $a_{11}$ component 
Fiber orientation distribution (a12)

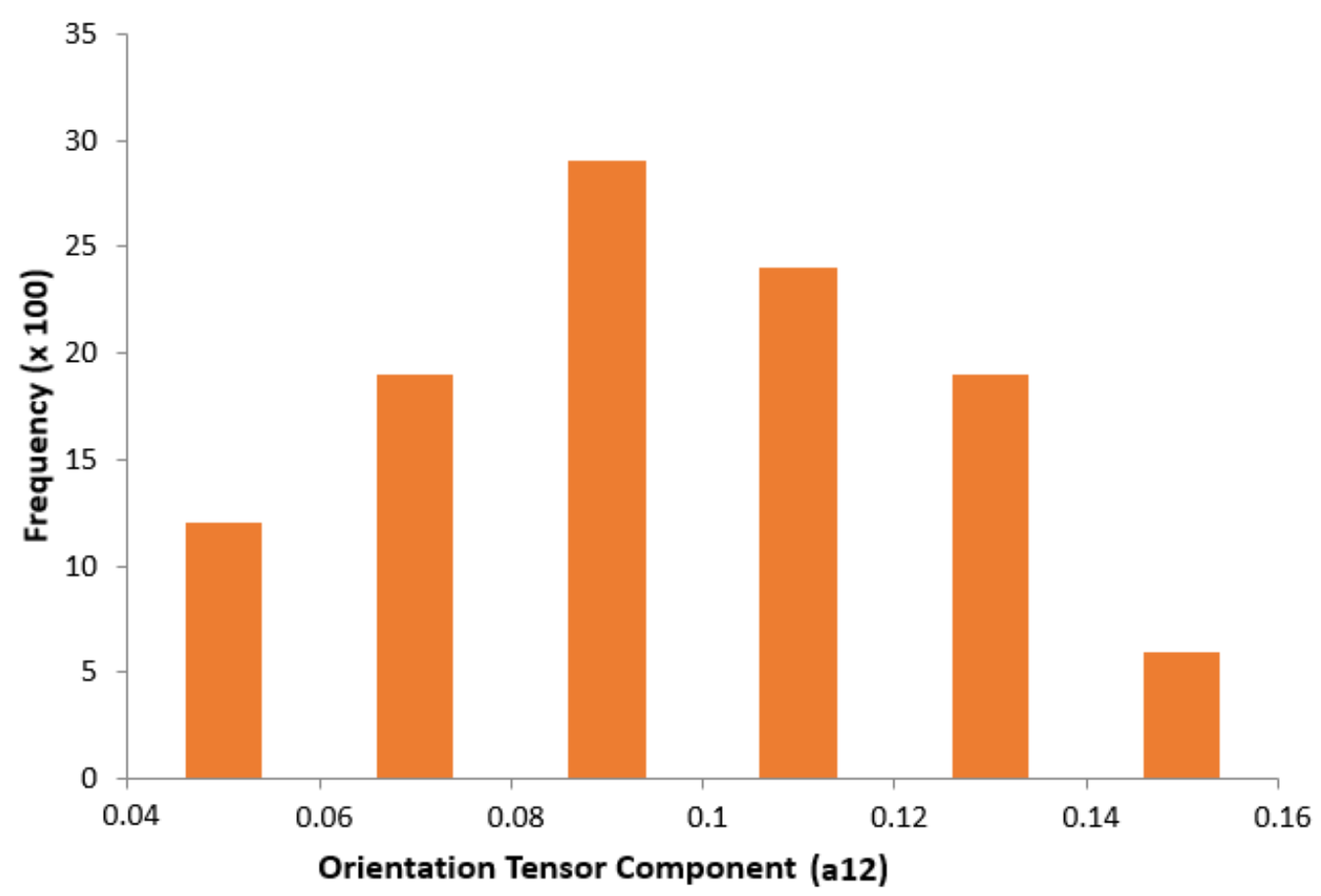

Figure 5-17 Fiber orientation distribution of $a_{12}$ component

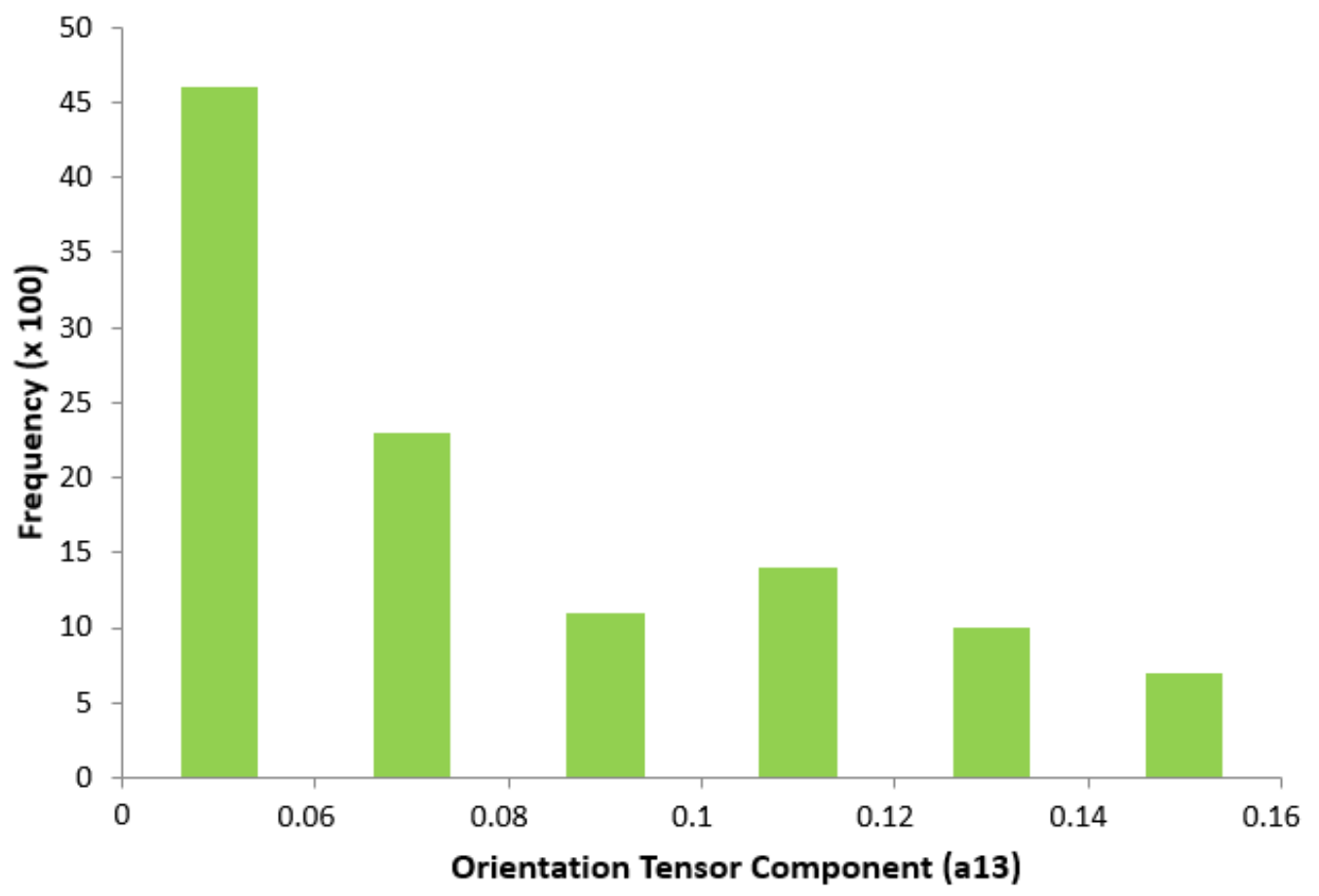

Figure 5-18 Fiber orientation distribution of $a_{13}$ component 


\subsection{Density measurement}

To investigated densities of parts, Nylon 12 samples printed by different layer heights and nozzle diameters were tested. Since $0.4 \mathrm{~mm}, 0.6 \mathrm{~mm}$, and $0.8 \mathrm{~mm}$ of nozzle diameters and $0.1 \mathrm{~mm}, 0.15 \mathrm{~mm}$, and $0.2 \mathrm{~mm}$ of layer heights are most commonly used parameters in $3 \mathrm{D}$ printing, samples printed by combinations of these parameters were examined. 3 samples of each case were printed for the repeatability; total 27 samples were prepared in the test as shown in Figure 5-19.

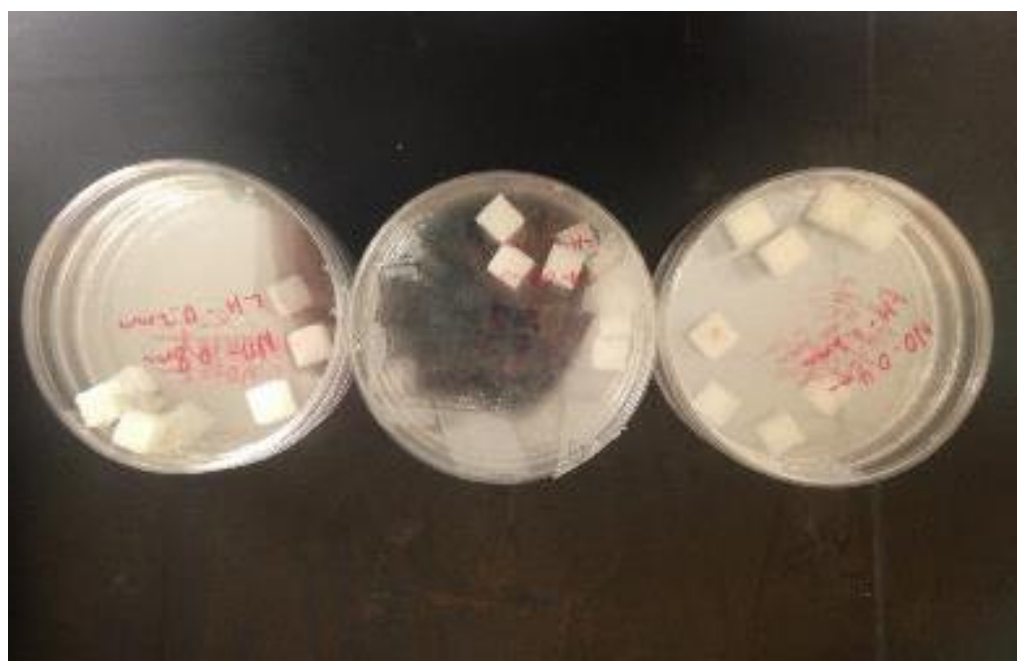

Figure 5-19 Cubic samples of Nylon 12

Archimedes's density method were applied. Ethanol was used as liquid. The size of samples was $10 \mathrm{~mm}^{\wedge} 3$ cubic. Samples were presumed as not isolated porous which means they have open voids. The procedure was as follows:

1. Measure dried weights of samples.

2. Submerge in ethanol $(0.789 \mathrm{~g} / \mathrm{cc})$ and vacumme the air_.

3. Measure suspended the weight of samples.

4. Wipeout ethanol with wet gauzes.

5. Measure the saturated weight of samples.

6. Compute density of samples using the equation $\rho_{\text {bulk }}=\frac{\omega_{\mathrm{d}} \cdot \rho_{1}}{\omega_{\text {sat }}-\omega_{\text {susp }}}$

7. Compute relative density of samples

Figure 5-20 shows the step 3 of Archimedes's density method. 


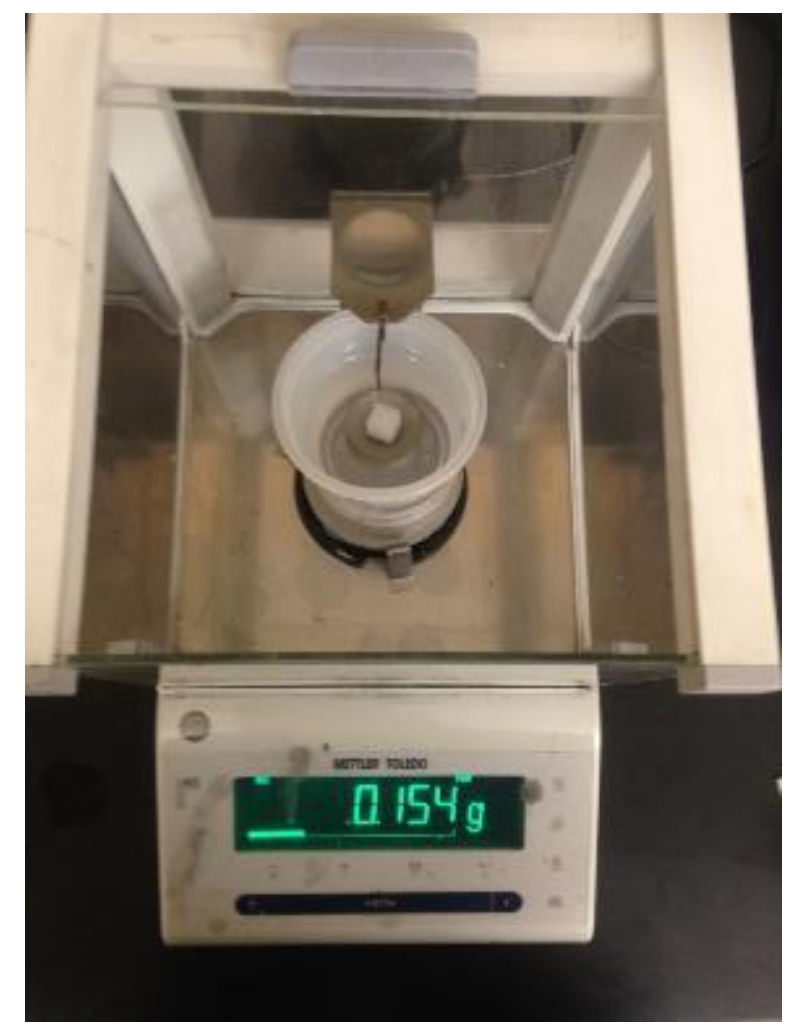

Figure 5-20 Archimedes's density measurement

Figure 5-21 shows relative densities of samples printed by various nozzle diameters and layer heights. Samples printed by $0.8 \mathrm{~mm}$ nozzle shows the highest density ranging from $85 \%$ to $97 \%$. Samples printed by $0.6 \mathrm{~mm}$ and $0.4 \mathrm{~mm}$ nozzle diameters show approximately $74 \%$ and $70 \%$ of densities, respectively. It is noted that samples printed by larger nozzle size increase densities. For the layer height, $0.15 \mathrm{~mm}$ shows the highest density for samples printed by $0.2 \mathrm{~mm}$ nozzle diameter. For the $0.4 \mathrm{~mm}$ nozzle size, however, $0.1 \mathrm{~mm}$ layer height shows the highest density. This means each nozzle size has its specific suitable layer height. Generally, low layer height reduces density because parts relatively need more layers when compared with the same size of parts. For high layer heights, however, parts need the small number of layers, but it may be hard to make rectangular cross-sections of beads as shown in Figure 3-8(b). Suitable layer heights are more required for large nozzle sizes such as $0.8 \mathrm{~mm}$ as illustrated in Figure 5-21. Table 5-3 shows experimental raw data of Archimedes's density test to support Figure 5-21. 


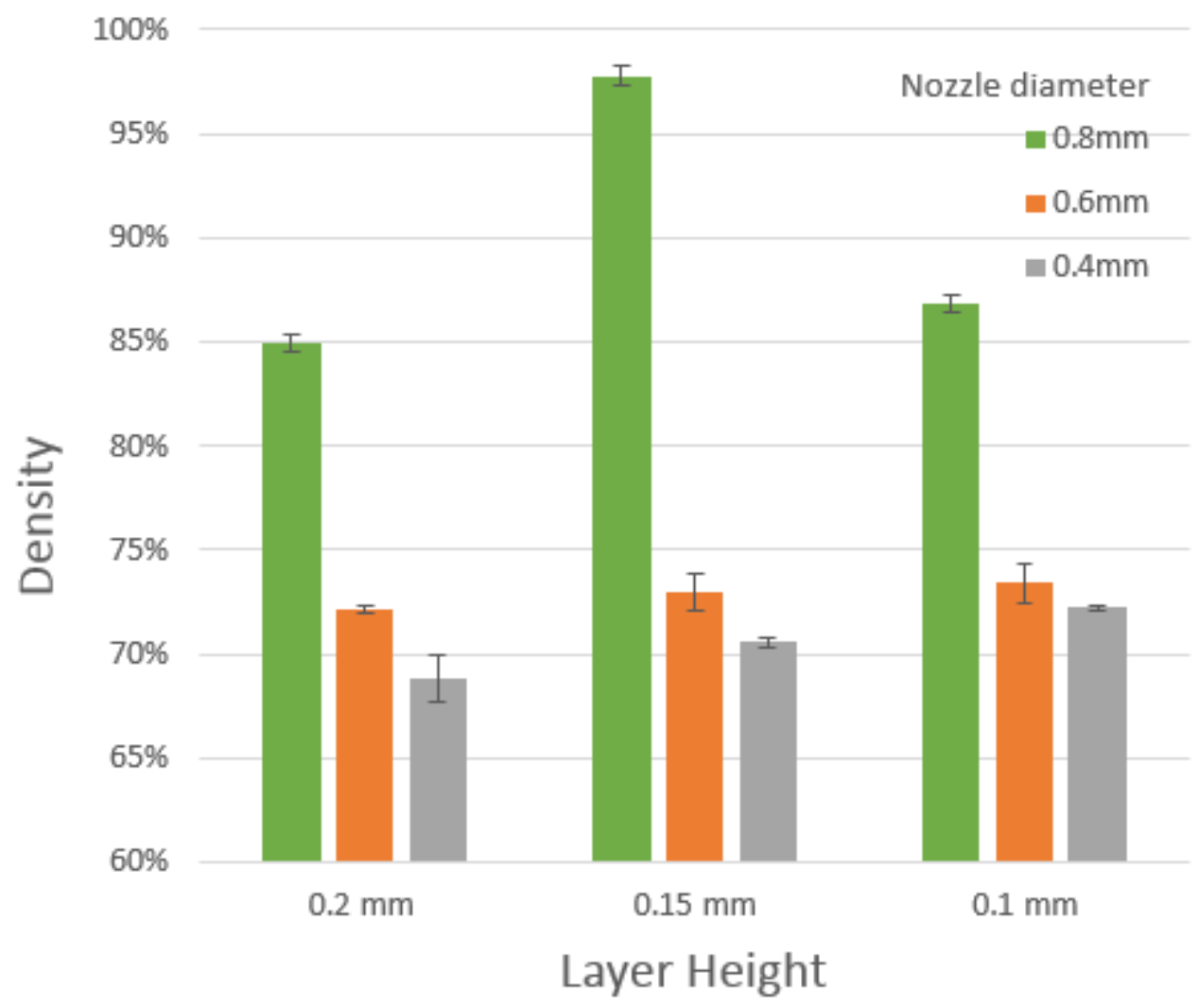

Figure 5-21 Relative densities of samples printed by various nozzle size and layer height

Table 5-3 Experimental data of Archimedies's density test

\begin{tabular}{l|rrr|rrr|rrr}
\hline & \multicolumn{2}{|c|}{$\mathrm{ND}=0.4 \mathrm{~mm}, \mathrm{LH}=0.2 \mathrm{~mm}$} & \multicolumn{2}{c|}{$\mathrm{ND}=0.6 \mathrm{~mm}, \mathrm{LH}=0.2 \mathrm{~mm}$} & \multicolumn{2}{c}{$\mathrm{ND}=0.8 \mathrm{~mm}, \mathrm{LH}=0.2 \mathrm{~mm}$} \\
\hline W_d(g) & 0.857 & 0.857 & 0.888 & 0.79 & 0.823 & 0.788 & 1.004 & 1.008 & 1.023 \\
W_susp & 0.158 & 0.151 & 0.192 & 0.171 & 0.18 & 0.16 & 0.241 & 0.244 & 0.245 \\
W_sat & 1.02 & 0.982 & 0.997 & 0.968 & 1 & 0.958 & 1.055 & 1.081 & 1.068 \\
p_bulk & 0.78 & 0.81 & 0.87 & 0.78 & 0.79 & 0.78 & 0.97 & 0.95 & 0.98 \\
Density & $68.81 \%$ & $71.38 \%$ & $76.35 \%$ & $68.60 \%$ & $69.46 \%$ & $68.34 \%$ & $85.37 \%$ & $83.35 \%$ & $86.03 \%$ \\
\hline & $\mathrm{ND}=0.4 \mathrm{~mm}, \mathrm{LH}=0.15 \mathrm{~mm}$ & $\mathrm{ND}=0.6 \mathrm{~mm}, \mathrm{LH}=0.15 \mathrm{~mm}$ & $\mathrm{ND}=0.8 \mathrm{~mm}, \mathrm{LH}=0.15 \mathrm{~mm}$ \\
\hline W_d(g) & 0.866 & 0.919 & 0.9 & 0.847 & 0.814 & 0.796 & 1.155 & 1.154 & 1.136 \\
W_susp & 0.177 & 0.186 & 0.18 & 0.16 & 0.153 & 0.136 & 0.339 & 0.339 & 0.344
\end{tabular}




\begin{tabular}{l|rrr|rrr|rrr} 
W_sat & 1.009 & 1.052 & 1.032 & 0.953 & 0.986 & 0.921 & 1.162 & 1.141 & 1.158 \\
p_bulk & 0.82 & 0.84 & 0.83 & 0.84 & 0.77 & 0.80 & 1.11 & 1.14 & 1.10 \\
Density & $72.04 \%$ & $73.45 \%$ & $73.11 \%$ & $73.92 \%$ & $67.63 \%$ & $70.18 \%$ & $97.13 \%$ & $99.59 \%$ & $96.59 \%$ \\
\hline & $\mathrm{ND}=0.4 \mathrm{~mm}, \mathrm{LH}=0.1 \mathrm{~mm}$ & $\mathrm{ND}=0.6 \mathrm{~mm}, \mathrm{LH}=0.1 \mathrm{~mm}$ & $\mathrm{ND}=0.8 \mathrm{~mm}, \mathrm{LH}=0.1 \mathrm{~mm}$ \\
\hline W_d(g) & 0.861 & 0.904 & 0.91 & 0.803 & 0.839 & 0.813 & 1.036 & 1.063 & 1.038 \\
W_susp & 0.162 & 0.17 & 0.175 & 0.052 & 0.17 & 0.047 & 0.27 & 0.258 & 0.26 \\
W_sat & 0.977 & 1.025 & 1.027 & 0.824 & 0.938 & 0.862 & 1.11 & 1.091 & 1.087 \\
p_bulk & 0.83 & 0.83 & 0.84 & 0.82 & 0.86 & 0.79 & 0.97 & 1.01 & 0.99 \\
Density & $73.12 \%$ & $73.18 \%$ & $73.92 \%$ & $71.99 \%$ & $75.61 \%$ & $69.04 \%$ & $85.36 \%$ & $88.32 \%$ & $86.87 \%$ \\
\hline
\end{tabular}

In order to visually verify the difference of densities between various conditions, SEM image of samples were captured. Samples printed by $0.4 \mathrm{~mm}, 0.6 \mathrm{~mm}$, and $0.8 \mathrm{~mm}$ nozzle diameters with suitable layer heights were prepared. 'suitable' means layer heights showing the best density for each nozzle diameter. For example, $0.15 \mathrm{~mm}$ layer height works with $0.8 \mathrm{~mm}$ nozzle size based on the data from Figure 5-21. Liquid nitrogen and dynamic hammer were utilized to see the fracture surface clearly without tensile deformation. Figure 5-22 shows a sample and the dynamic hammer used to break samples. Also, the same magnitude of 70x was applied to visually compare void contents between samples accurately.
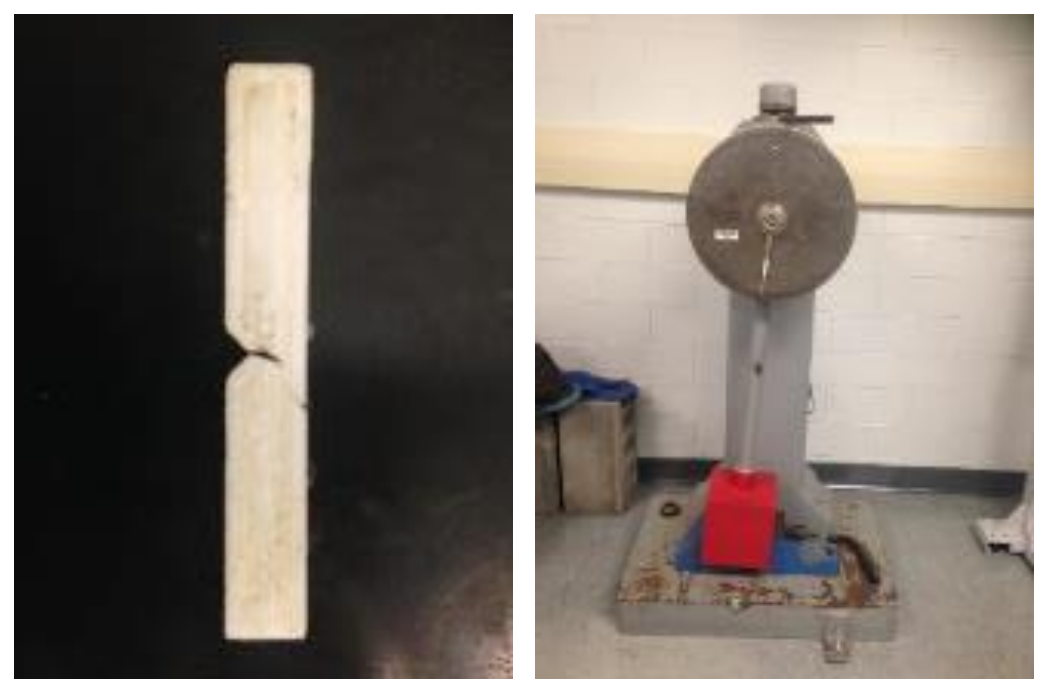

Figure 5-22 samples for SEM and dynamic hammer 
Figure 5-23, Figure 5-24, and Figure 5-25 show SEM images of samples printed by $0.4 \mathrm{~mm}, 0.6 \mathrm{~mm}$, and $0.8 \mathrm{~mm}$ of nozzles, respectively. They show details of the periodic microstructure of the part, including triangular shaped voids left by the process. As shown, samples printed by smaller nozzles show some small voids for the same area of one spot. For samples printed by $0.8 \mathrm{~mm}$ nozzle, however, it shows the small number of tiny voids. These SEM images support the test results of bulk density experiments.

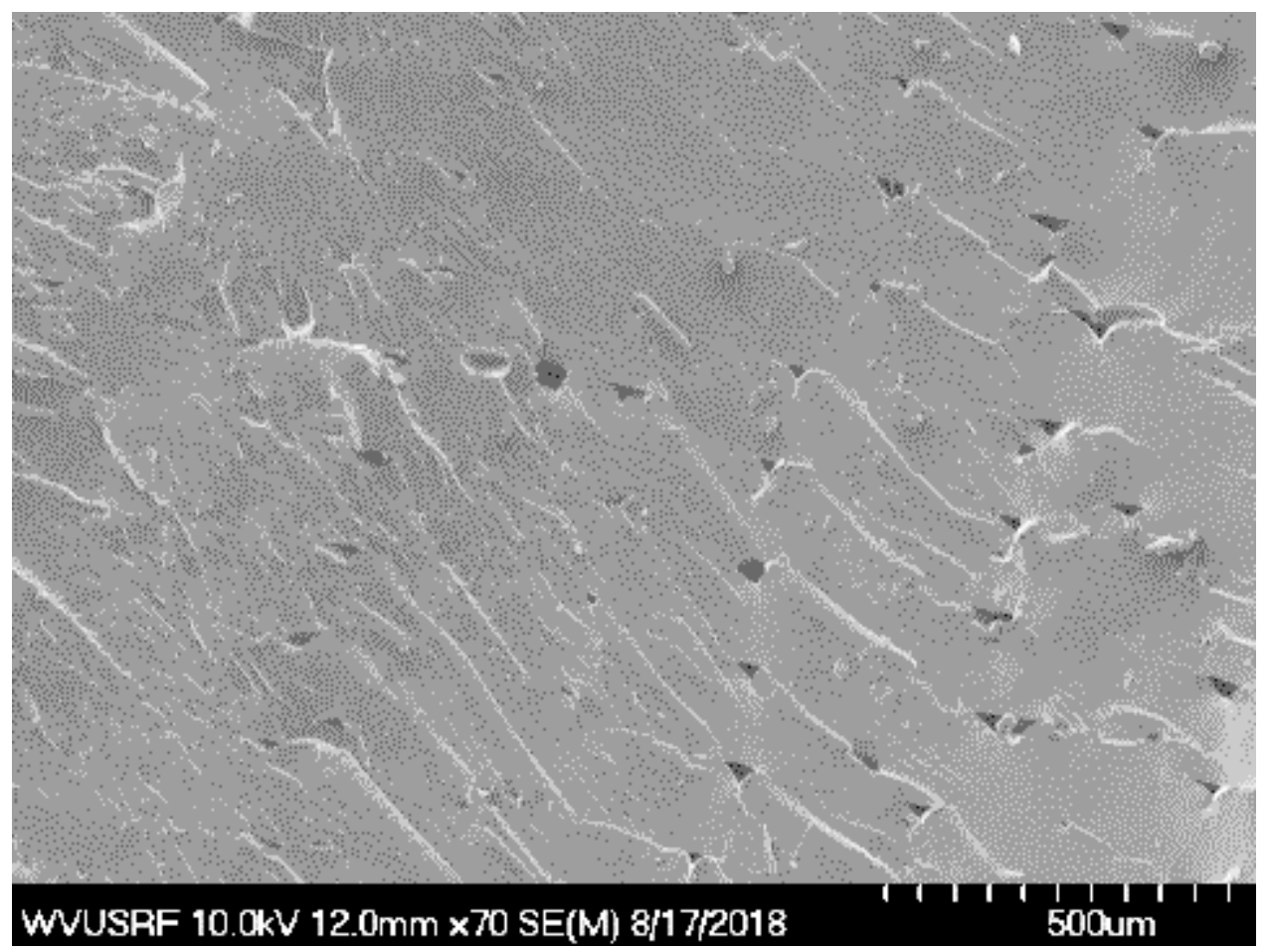

Figure 5-23 SEM image of a sample printed by $0.4 \mathrm{~mm}$ nozzle with $0.1 \mathrm{~mm}$ layer height 


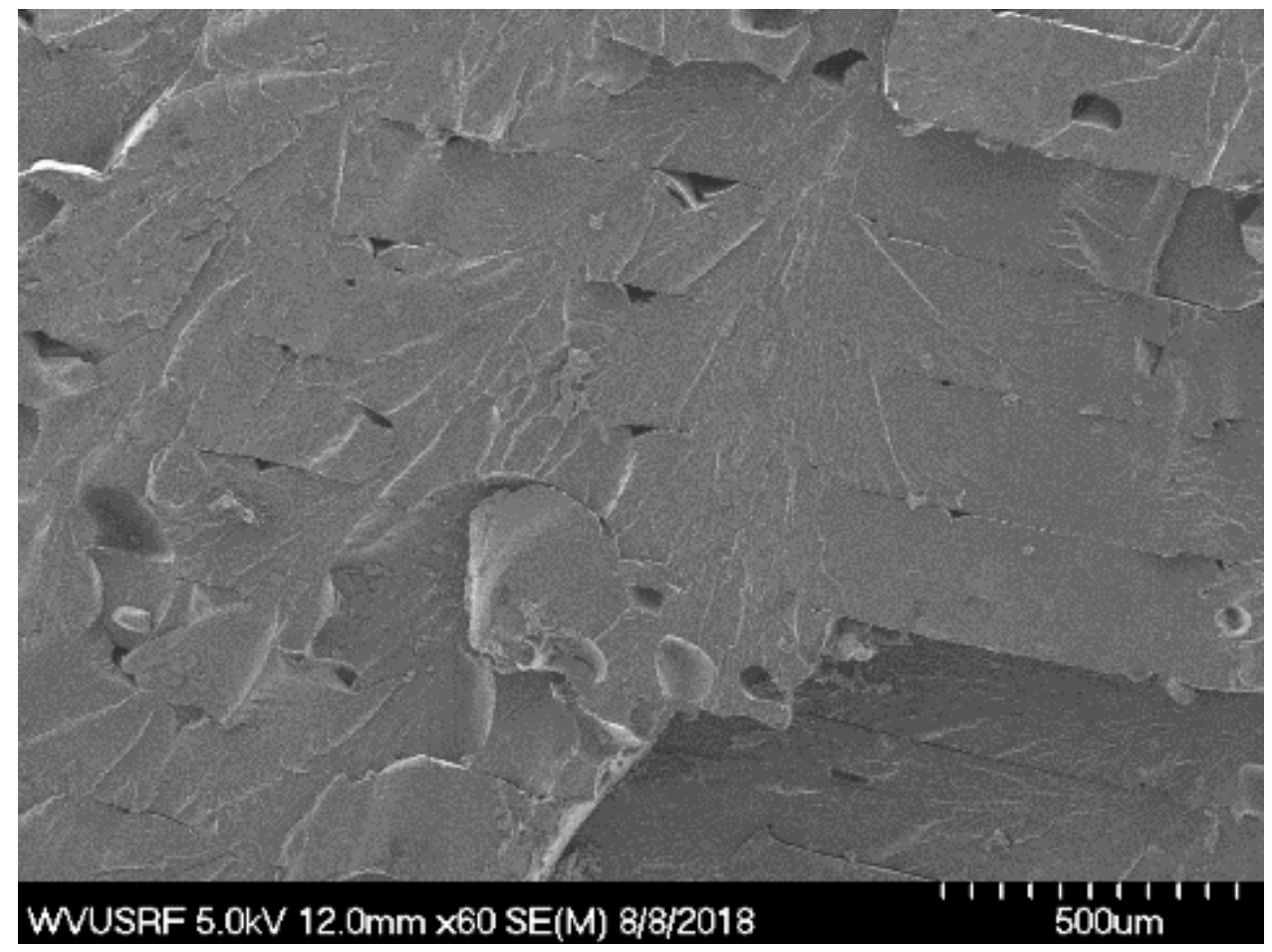

Figure 5-24 SEM image of a sample printed by $0.6 \mathrm{~mm}$ nozzle with $0.15 \mathrm{~mm}$ layer height

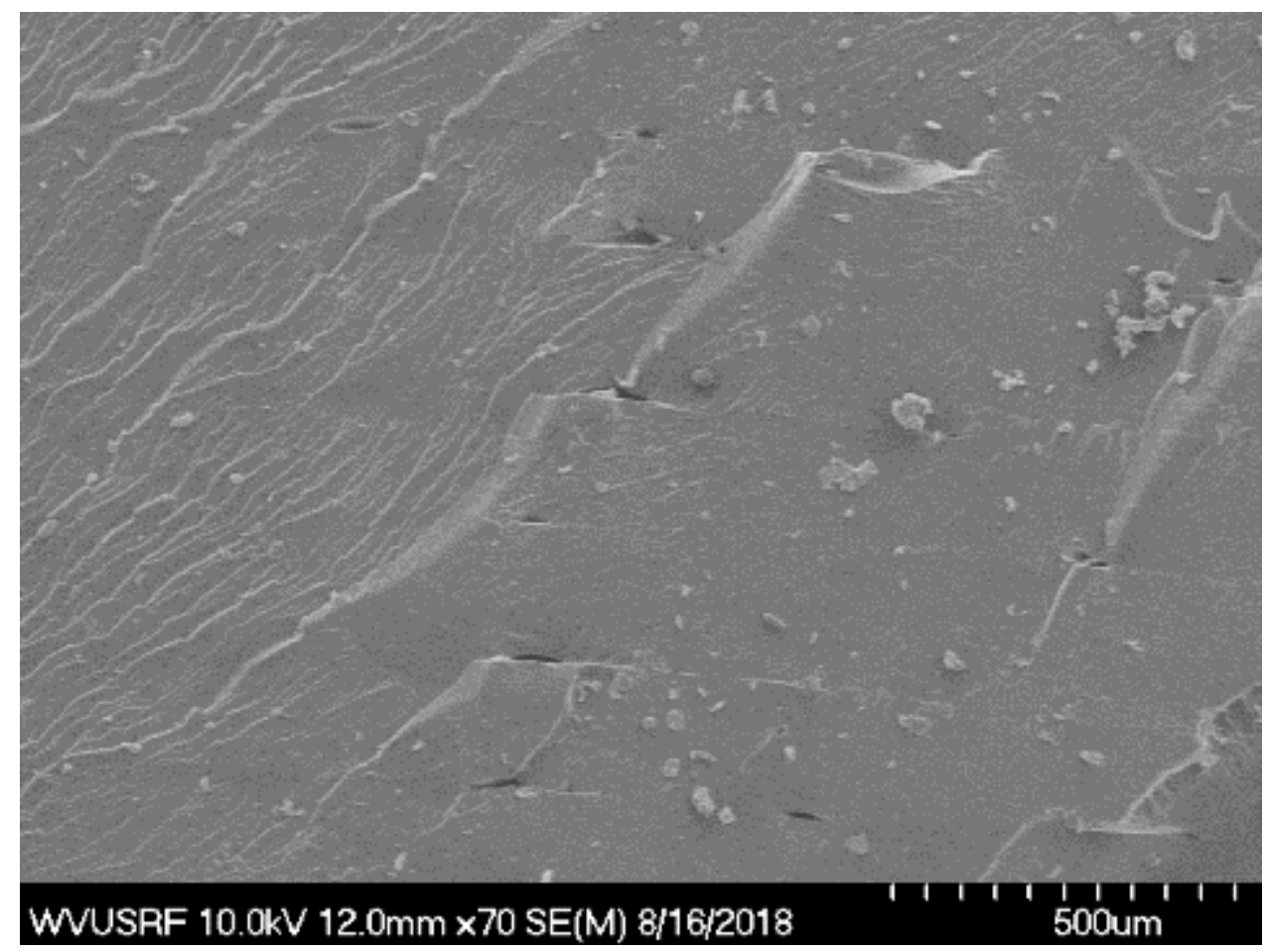

Figure 5-25 SEM image of a sample printed by $0.8 \mathrm{~mm}$ nozzle with $0.15 \mathrm{~mm}$ layer height 
Tensile tests were performed to see how densities correlate with mechanical properties of parts produced by FFF. Figure 5-26 shows stress-strain curves from tensile tests of samples printed by various nozzle sizes. The test result shows no big difference in stiffness between samples. However, samples printed by $0.4 \mathrm{~mm}$ and $0.8 \mathrm{~mm}$ nozzles show approximately $27 \%$ and $10 \%$ lower failure strength compared with samples printed by $0.6 \mathrm{~mm}$. In addition, as nozzle size increases, the higher plastic strain shows up. For samples printed by $0.8 \mathrm{~mm}$ nozzle, it shows the highest elongation which is very close with the theoretical value of Nylon 12. Figure 5-14 shows the failure of one of samples printed by $0.8 \mathrm{~mm}$ nozzle. Even if using $0.8 \mathrm{~mm}$ nozzle diameter takes an advantage in elongation and shows better density, $0.8 \mathrm{~mm}$ nozzle can't print parts precisely if the object is handy size. This may cause a lots of defects. That's the reason why $0.6 \mathrm{~mm}$ nozzle size is preferred in $3 \mathrm{D}$ printing industry. However, if the object is big enough $(20 \mathrm{~cm}$ x $20 \mathrm{~cm}$ x $20 \mathrm{~cm}), 0.8 \mathrm{~mm}$ nozzle may better work.

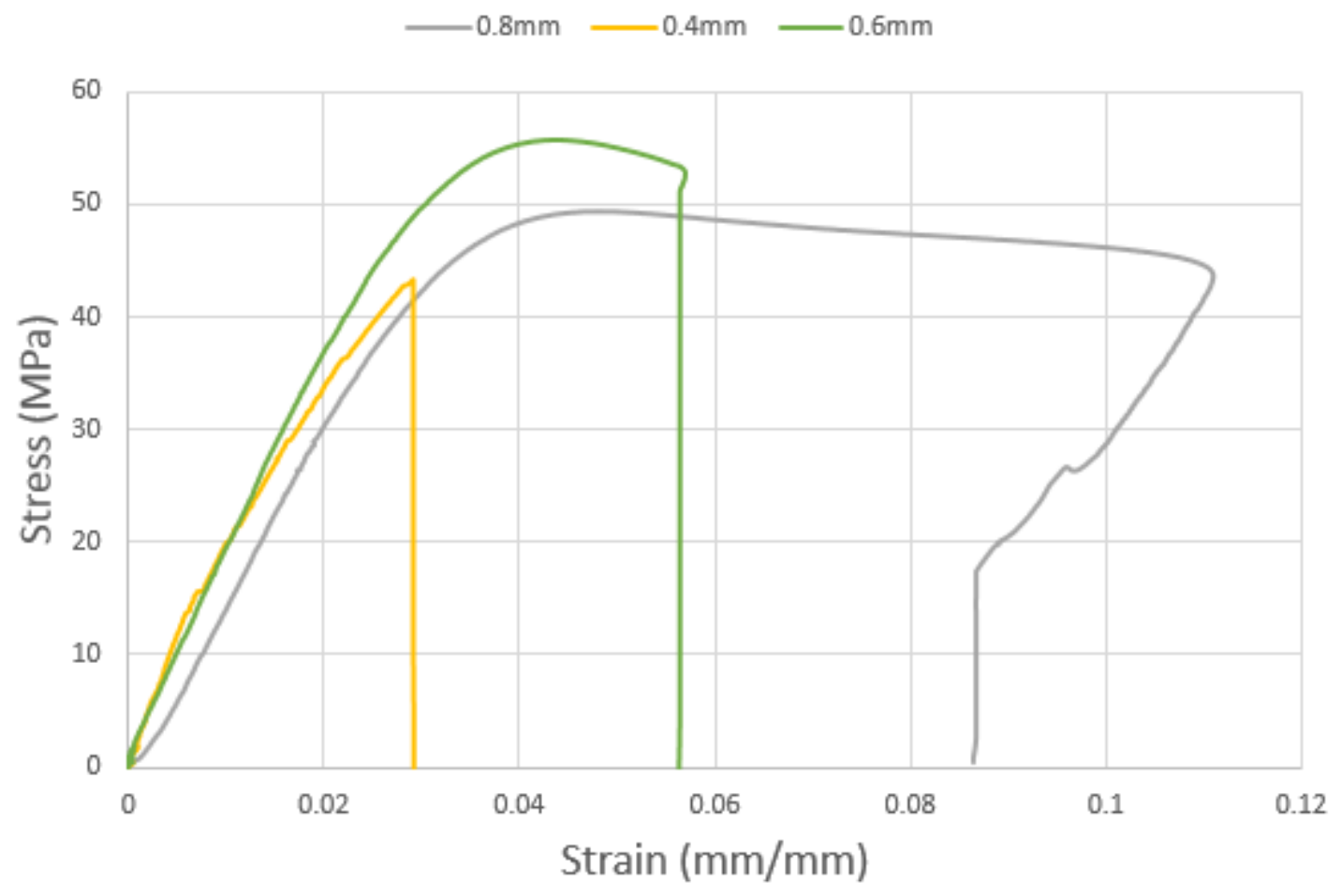

Figure 5-26 Tensile test results of samples printed by various nozzle sizes 


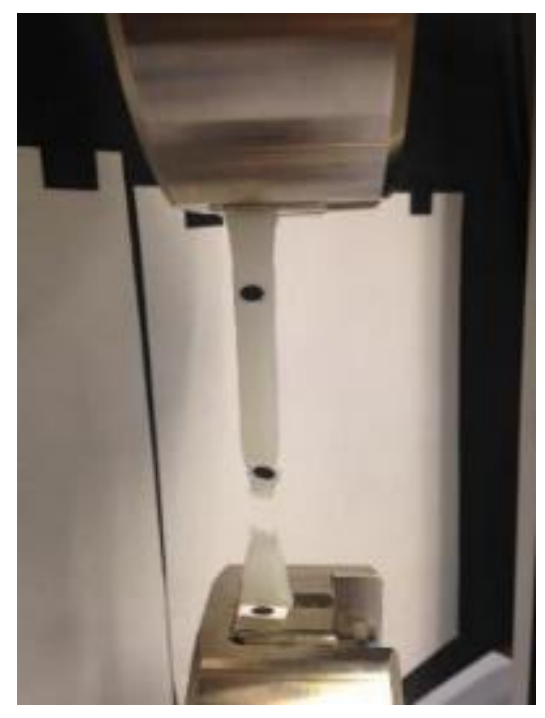

Figure 5-27 Tensile failure of a specimen printed by $0.8 \mathrm{~mm}$ nozzle

\subsection{Microstructure of CFRP}

Figure 5-28 and Figure 5-29 are micrographs obtained by SEM, showing the cross-section of a sample of the CFRP-nylon with printing direction $0^{\circ}$. From Figure 5-28 with a magnification of 150x, it is possible to see the round shape of the carbon fiber cross-section, whose nominal diameter 6 um. In Figure 5-29, with a magnification of $250 \mathrm{x}$, it is possible to notice the same elements found in the previous micrographs: carbon fibers, voids left by fiber pull-outs and other voids. It is clearly seen that the filament has the short carbon fibers mostly oriented with the direction of its length. Therefore, it can be safely assumed that the carbon fibers were aligned within the feeding filament and remained aligned in the tested specimens produced by the FFF process. This behavior can be explained by the fact that, during 3D printing, the nylon is meltdown by the extrusion nozzle and the fibers inside the deposited material have a trend to become aligned with the extrusion direction of the molten thermoplastic. This behavior was already reported in the literature [70][54] for ABS reinforced with short carbon fibers, and is now also observed for nylon. However, in an injection molded nylon with the addition of very short carbon nanofibers at [77] this was not observed, leading to conclude that this alignment is a function of the production process and possibly of the fibers geometry. The voids that are not related to fiber pull-outs are also seen in the CFRP filament, the reason for the appearance of these voids is possibly due to a manufacturing defect during the $3 \mathrm{D}$ printing. 


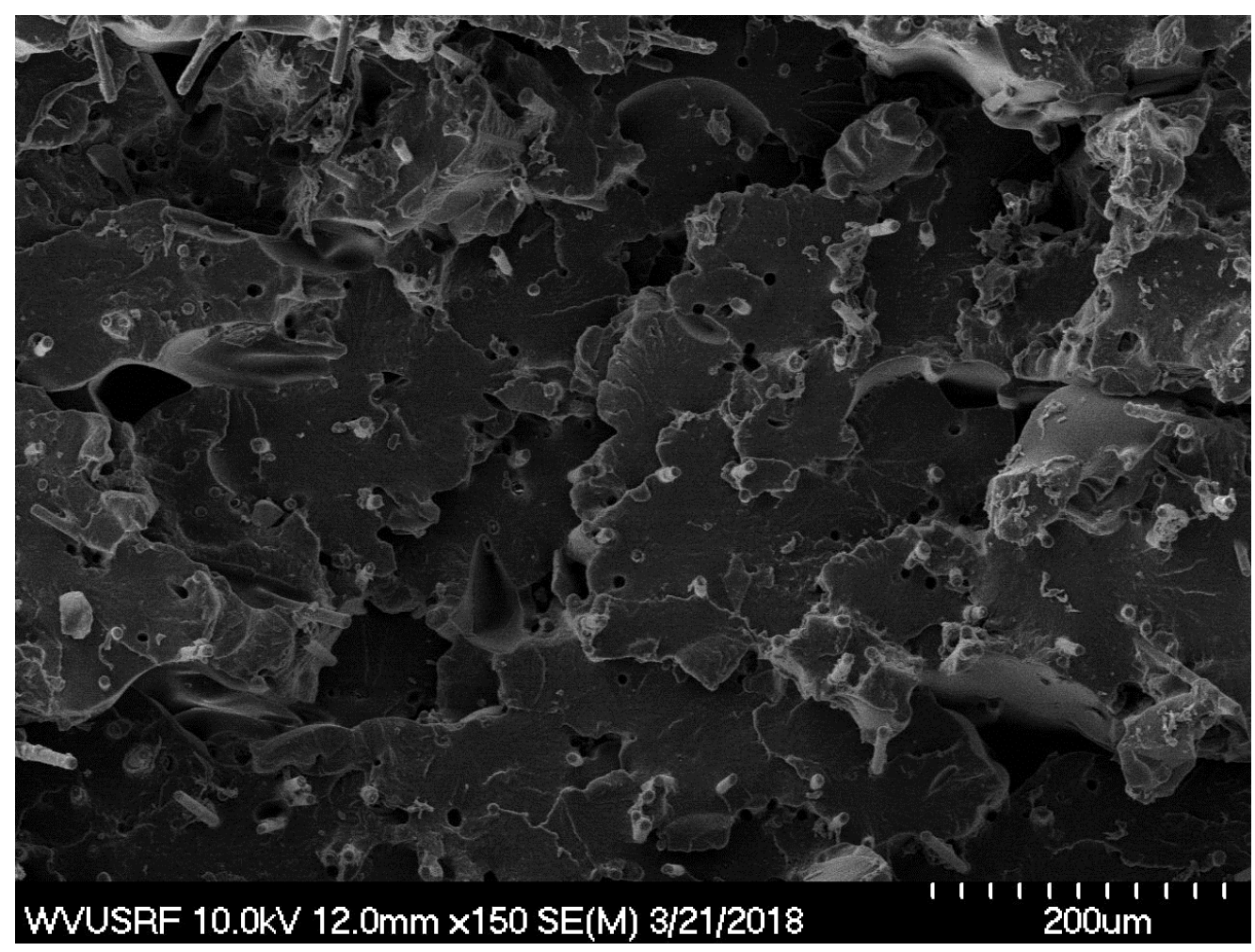

Figure 5-28 SEM image of CFRP specimen printed at $0^{\circ}$ direction (150x)

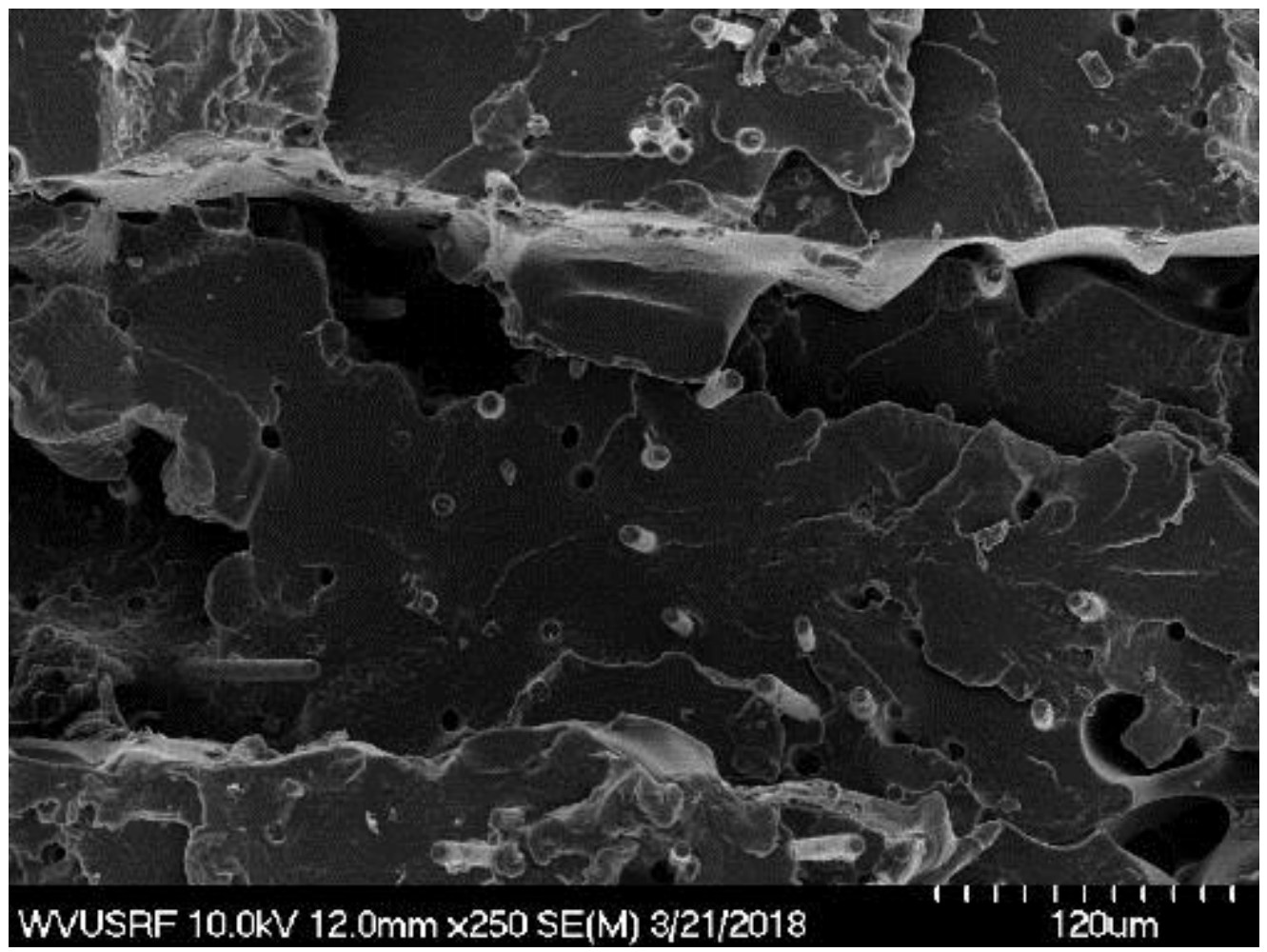

Figure 5-29 SEM image of CFRP specimen printed at $0^{\circ}$ direction (250x) 
Figure 5-30 and Figure 5-31 show the SEM images of the CFRP specimens that the short carbon fibers dispersed in the nylon matrix showed up to be highly oriented with the printing directions $45^{\circ}$ and $90^{\circ}$, respectively. This is the fact that explains the differences in stiffness noticed in the results of Table 5-1 It can be concluded that the CFRP-nylon printed material has a stiffness behavior similar to unidirectional fiber reinforced composites, once it is indeed a composite reinforced with short fibers which ended up highly oriented with the printing direction after production by FFF. Therefore, it concluded that the fiber orientation could be fully controlled by extruding, not is already oriented in the filament loaded in 3D printer.

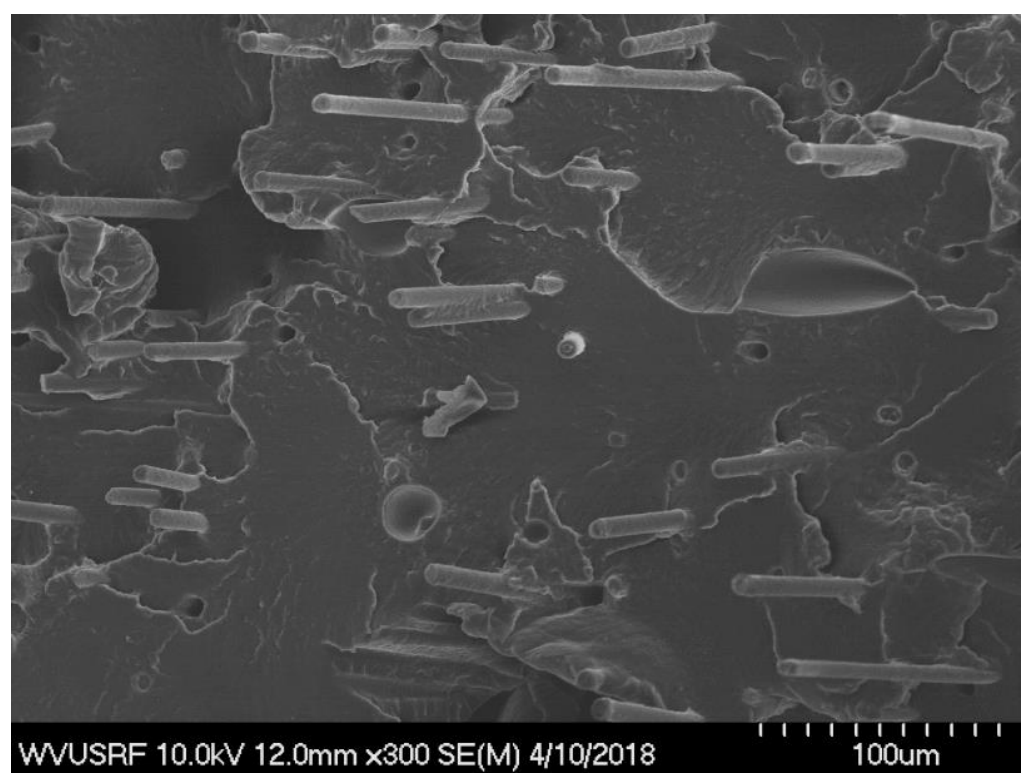

Figure 5-30 SEM image of CFRP specimen printed at $45^{\circ}$ direction

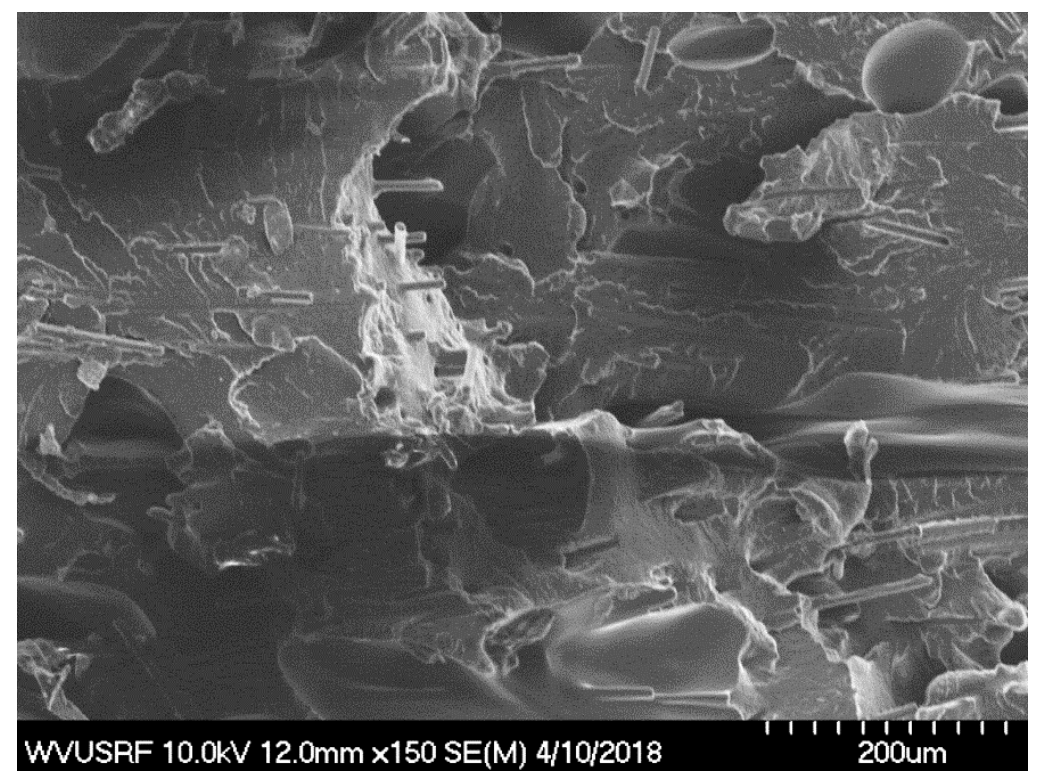

Figure 5-31 SEM image of CFRP specimen printed at $90^{\circ}$ direction 


\section{Chapter 6 CASE STUDY}

\subsection{Case Study I - Lattice Structure}

\subsubsection{Introduction}

From the literature in Chapter 2.1, it is noted that final topologies of structural optimizations are mostly some kind of lattice structures. Those lattice structures with complex geometries now can be easily manufactured by additive manufacturing. Up to now, a wide variety of industrial applications for lattice structures has been achieved. Figure 6-1 shows an unmanned aerial vehicle (UAV) with an optimized frame. Four arms of the quad-copter are optimized as truss structures. UAV design generally requires lightweight and high structural performance. In this chapter, structural optimization of a simply supported beam under bending loading is reviewed, and a novel manufacturing method of FFF 3D printing with fiber reinforcement is developed.

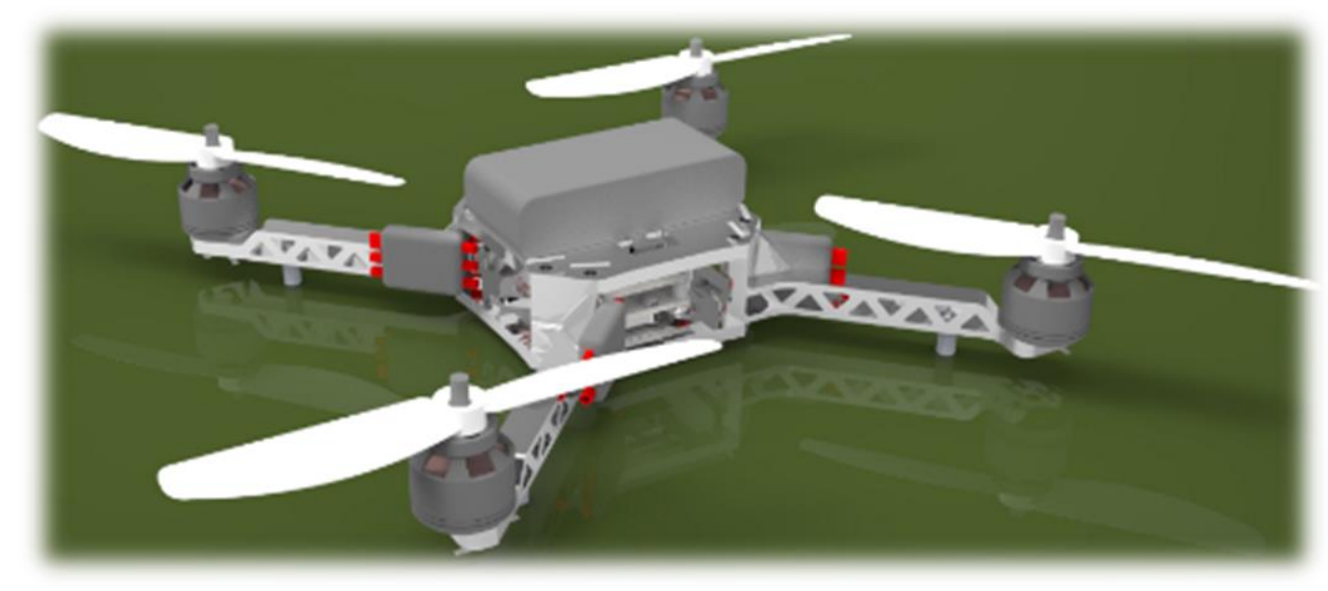

Figure 6-1 Quad-copter with an optimized frame [117]

\subsubsection{Structural Optimization}

Figure 6-2 shows an example considering stiffness optimization of a simply-supported beam under a concentrated loading. The design domain has length $100 \mathrm{~mm}$, height $10 \mathrm{~mm}$, and thickness $1 \mathrm{~mm}$, the force is applied downward in the middle of the beam with the magnitude of $100 \mathrm{~N}$. The material has Young's modulus of $100 \mathrm{GPa}$ and Poisson's ratio of 0.3 , and the available material can only cover $40 \%$ volume of the design domain. BESO starts from the full design which is subdivided using a regular mesh of size $100 \times 10$, totaling 1000 four-node quadrilateral elements. The BESO parameters are evolution rate $=1 \%$, $\mathrm{R}=3 \mathrm{~mm}$ and tolerance $=0.01 \%$. 


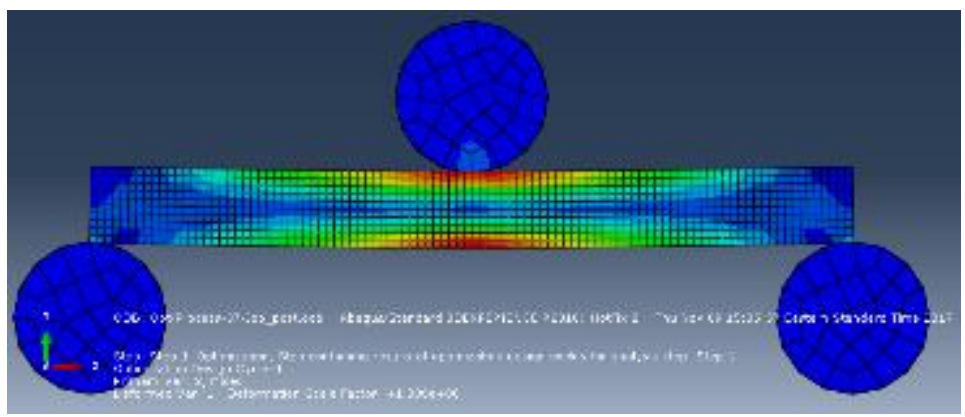

Figure 6-2 An example of topology optimization for a simply-sported beam subjected to three points bending

Figure 6-3 shows the evolution histories of the mean compliance and the volume fraction. The mean compliance increases as the material is gradually removed from the design domain. It is noted that apparent couple bumps in the mean compliance are caused by the significant effect of a change of the topology resulting from bar elimination. Thereafter, the mean compliance is quickly recovered and assures that the topology develops in the right direction. After the volume reaches the objective volume, the mean compliance is convergent to a constant.

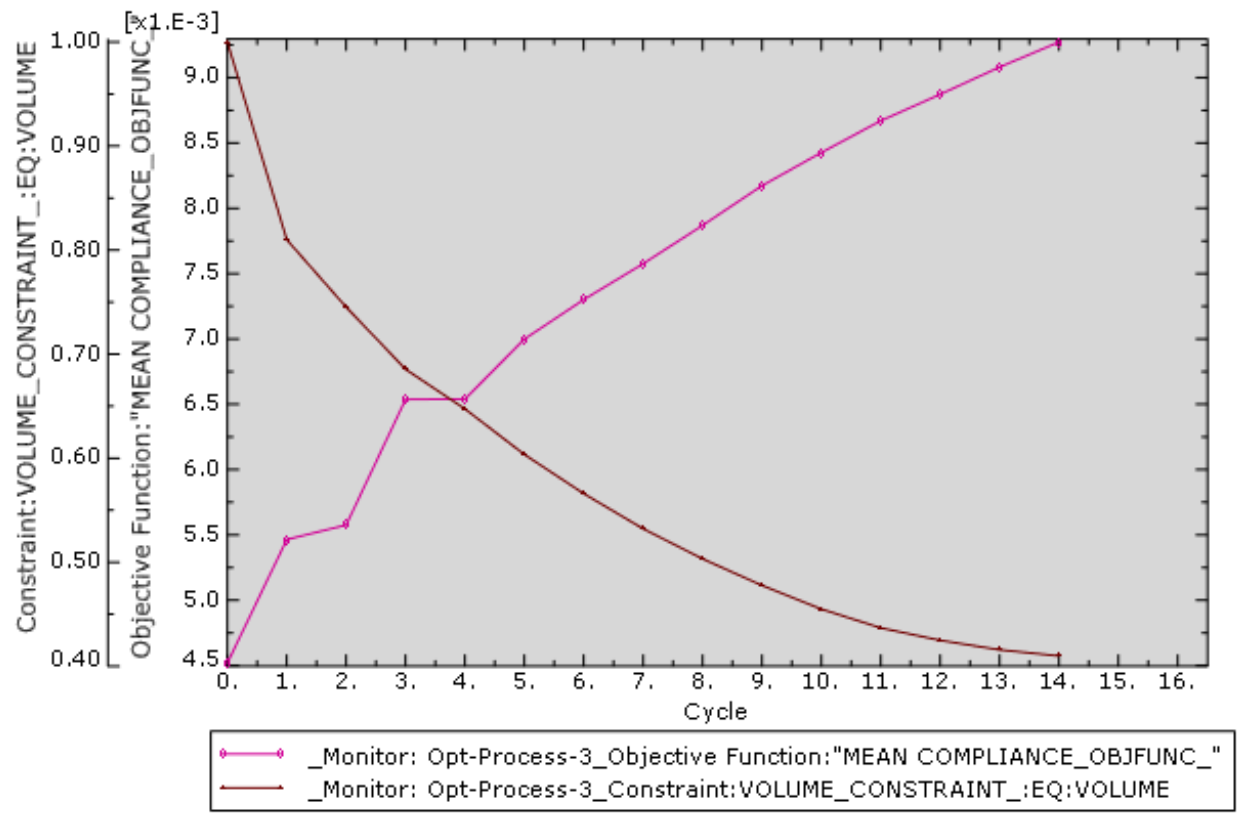

Figure 6-3 Evolution history of volume fraction and global stiffness

Figure 6-4 shows the evolution of topology where the final topology is shown in Figure 6-4(c). The optimization procedure will be stopped after 14 iterations. 

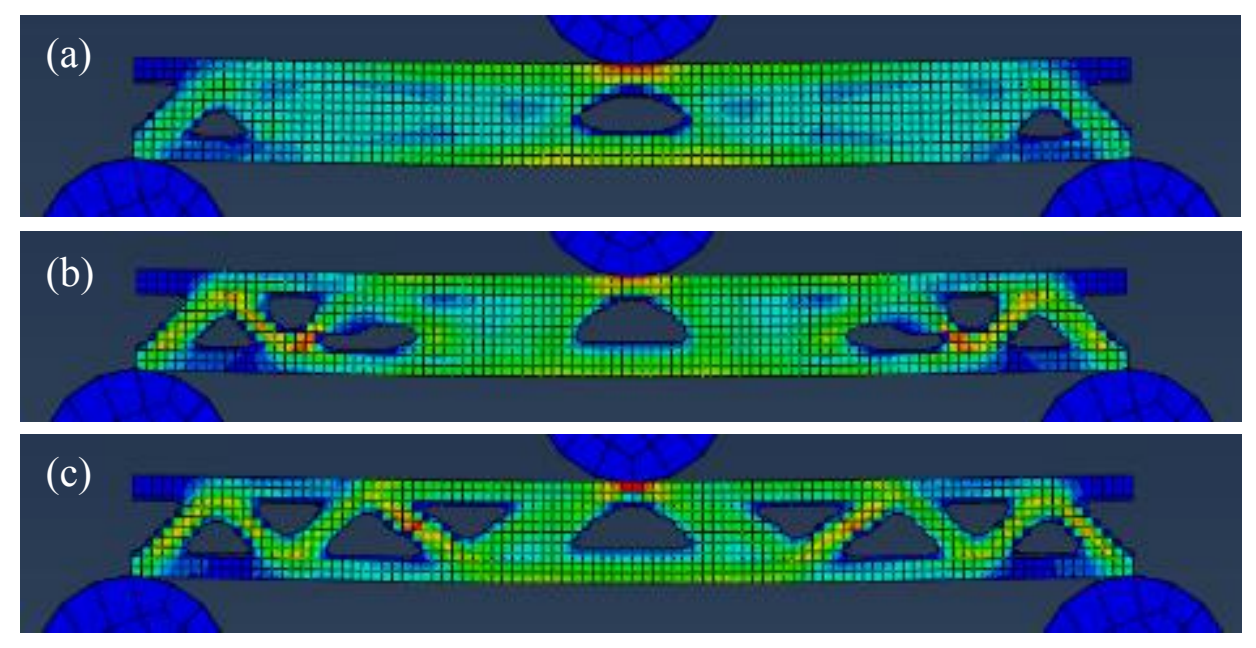

,Figure 6-4 Evolution of the topology: (a) iteration 5, (b) iteration 11 and (c) final topology.

Once structural optimization finished, principal directions of individual elements using stress tensor output from ABAQUS were computed. Figure 6-5 shows an example of optimal fiber angle distribution. Using statistics from the information on principal directions several representative fiber angles can be determined. In this research, $+45^{\circ}, 0^{\circ},-45^{\circ}$ were chosen as representative fiber angles for simplicity. Any elements that have the difference of angle value within $\pm 45 / 2^{\circ}$ with the computed representative angles create a section and nodes boundaries are generated. This procedure was numerically carried out by MathLAB 2016. Figure 6-6 describes a model of section division. The computed representative fiber angle values will be utilized as inclinations of infill pattern in the tool-path development.

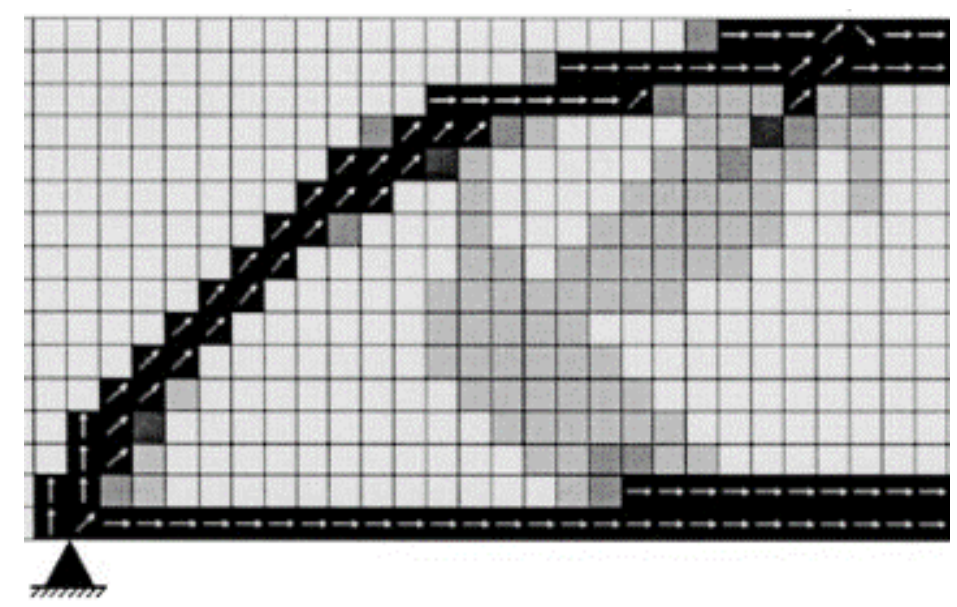

Figure 6-5 An example of optimal fiber angle distribution [109] 


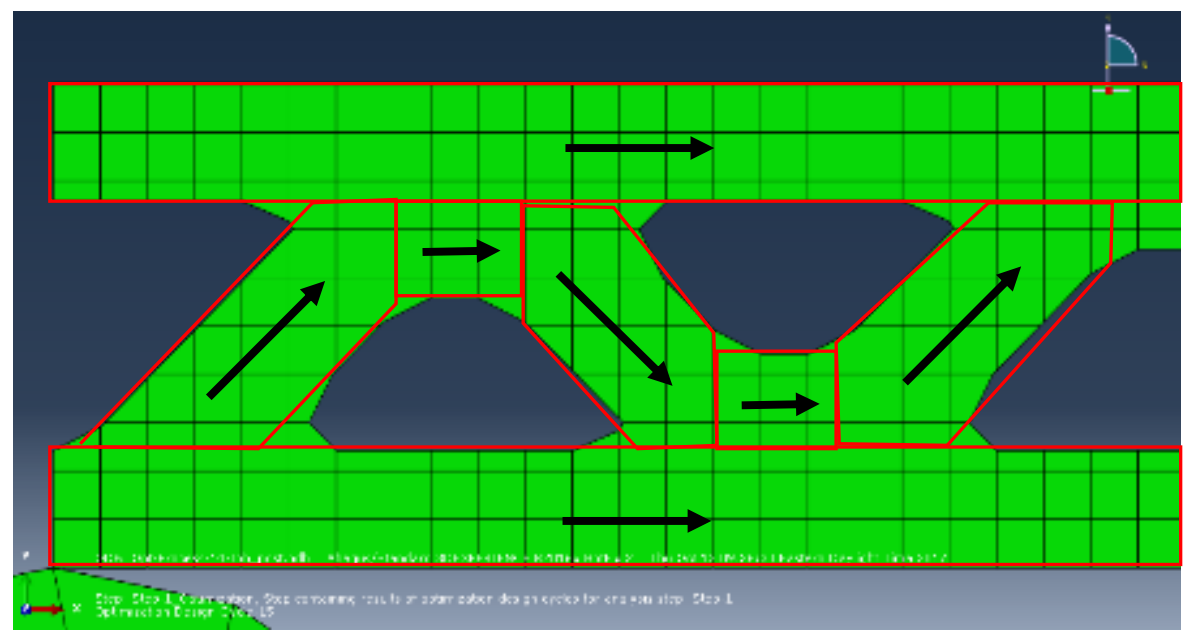

Figure 6-6 Section division for customized tool-path

\subsubsection{Tool-path Development}

As previously mentioned, there are two different types of infill patterns. In this case, direct parallel toolpath method is applied to achieve the designed fiber distribution with optimal orientations. The directionparallel tool-path is one of the most common tool-paths employed in current FFF techniques. This method fills an area line-by-line in a specified direction. After determining the inclination of the reference lines, a series of line segments (along the predefined inclination) connected with small turn segments are generated as the tool-paths as shown Figure 6-7

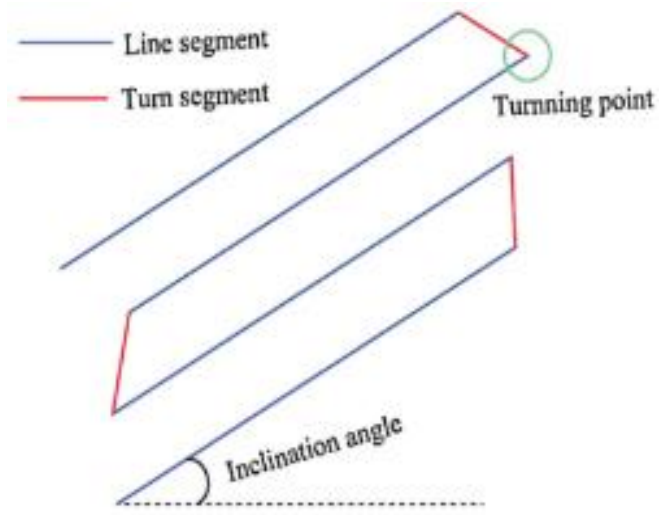

Figure 6-7 Illustration showing a direction-parallel tool-path segments [118]

In this manufacturing step, basically two different requirements must be satisfied. The first is the fiber orientation. The second is product quality. In order to meet the first requirement, nozzles with small diameter should be used to shorten the length of turn segment. However, the presence of vast numbers of 
small turn segments in the tool-paths can degrade the fabrication quality and efficiency to some extent. Also, small size nozzles like $0.25 \mathrm{~mm}$ and $0.4 \mathrm{~mm}$ don't fully work for carbon fiber reinforced filaments. Those nozzles are frequently clogged with filaments since fibers are not melted during the extrusion. Since carbon fiber filament manufactures don't specify recommended nozzle sizes, information from some 3D printer communities based on engineers' experience was used. In this research $0.6 \mathrm{~mm}$ is utilized throughout the manufacturing. In Figure 6-8, horizontal boundary of material deposition indicates nozzle diameter.

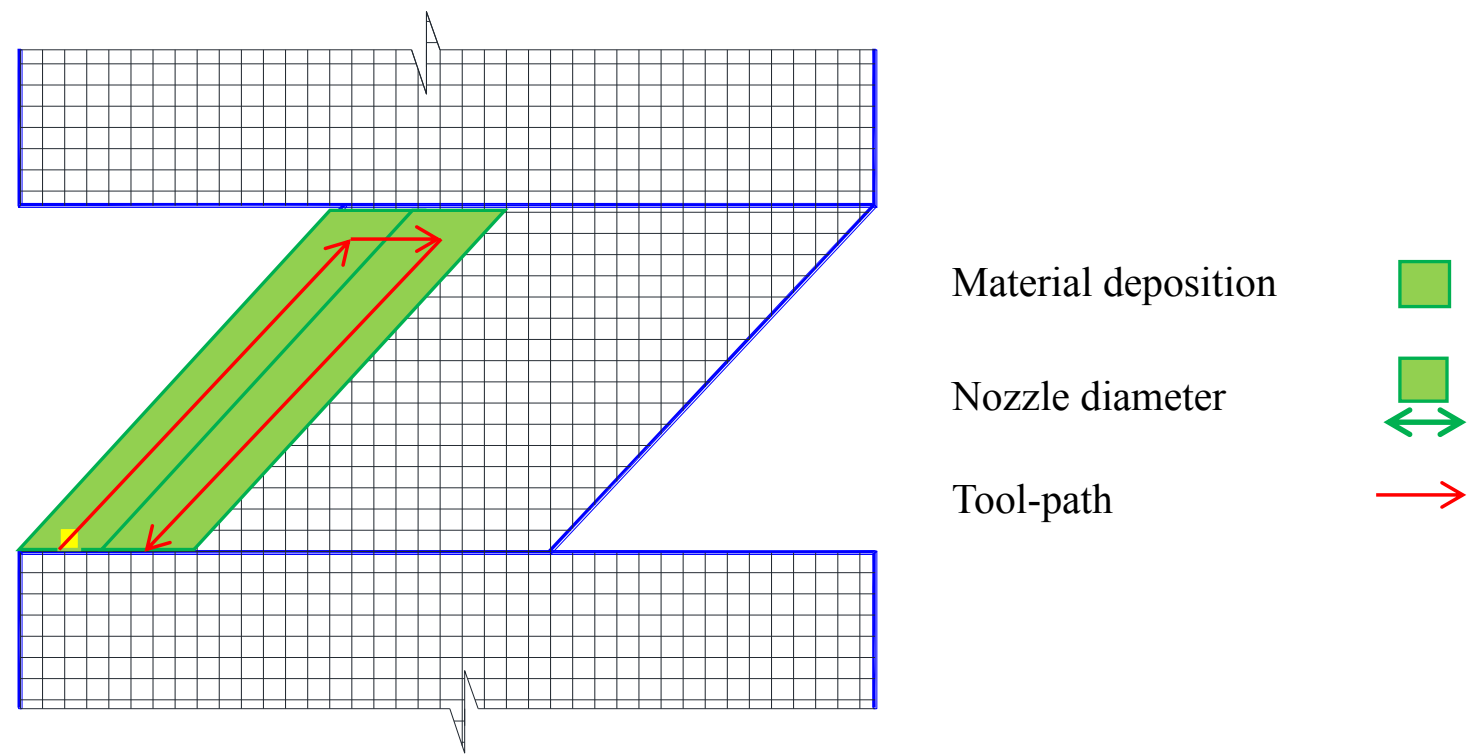

Figure 6-8 Material deposition scheme of FFF 3D printing for a section

Figure 6-9 shows a captured image of the tool-path simulation for the optimized structure with desired fiber orientations. Red line indicates extruder moving. This job was performed by open-source software Simplify3D 4.0.

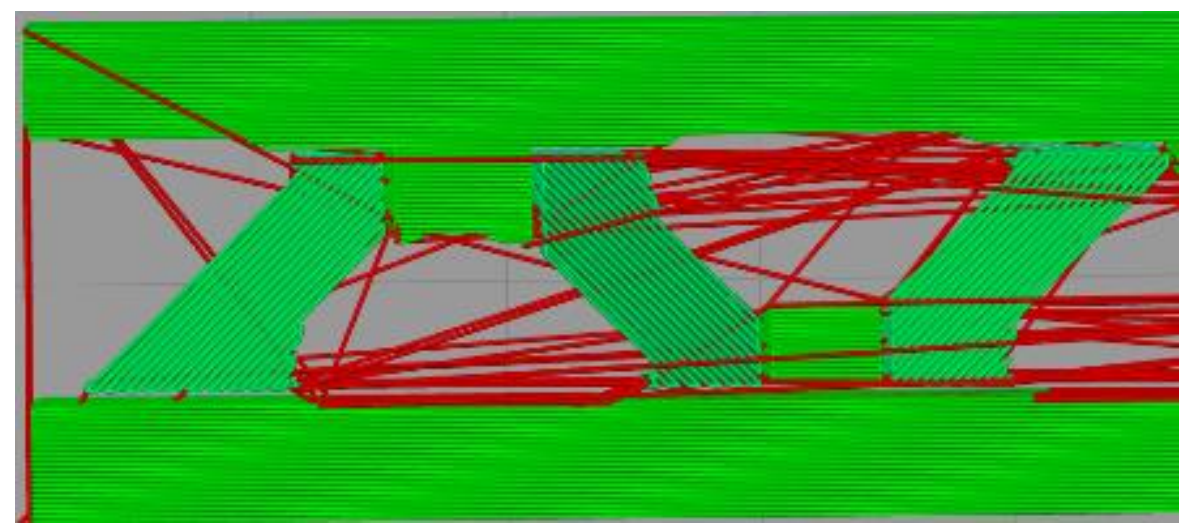

Figure 6-9 Customized tool-path for the optimized structure 


\subsubsection{FE modeling and test simulation}

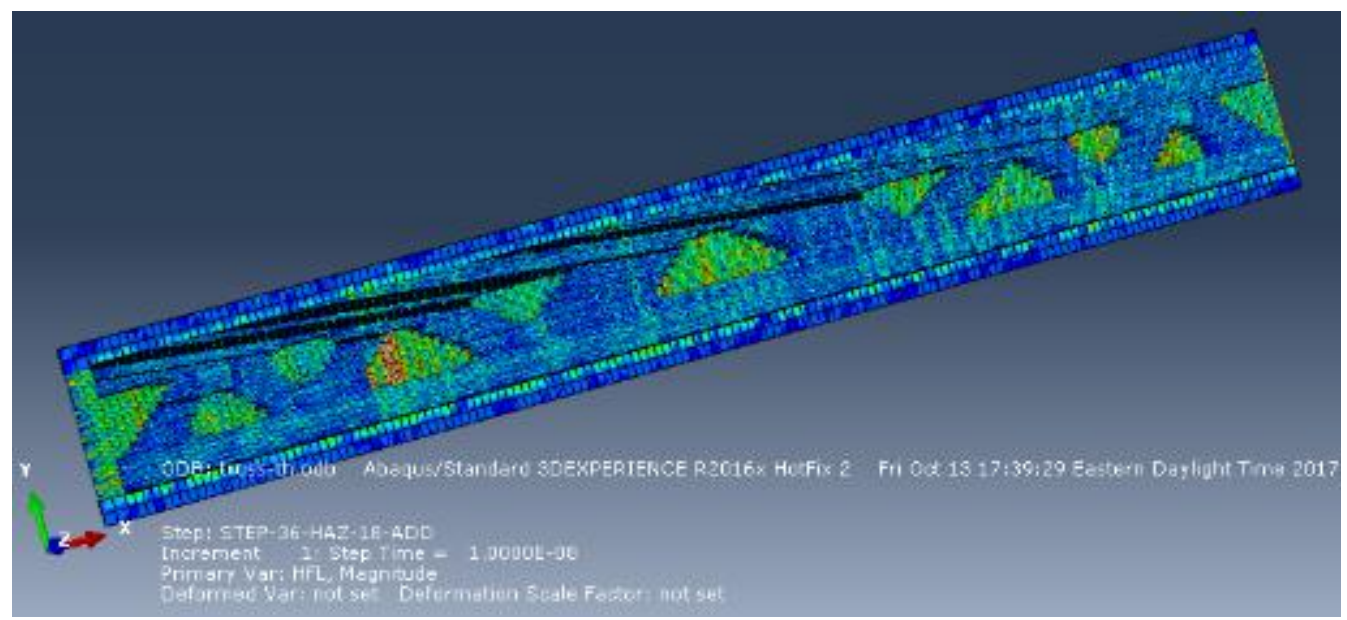

Figure 6-10 FE modeling of the optimized beam

The orthotropic mechanical properties of CFRP from the experimental data was applied to the optimized beam. As discussed previously, G-codes of outer shell tool-path was used to draw the part. 200 division number of $\mathrm{X}$ and $\mathrm{Y}$ coordinates and only one layer were applied to reduce computation time. Total $200^{*} 200=40,000$ number of elements were generated. Material orientation ranging from $-90^{\circ}$ to $+90^{\circ}$ was applied to individual elements. Since part size is handy, residual stress was not analyzed. Figure 6-10 shows FE modeling of the optimized beam. Structural performances of optimized structures with different fiber reinforcements are reviewed by 3 points bending test. For the comparison, structures with uniform fiber orientations $0^{\circ}, 45^{\circ}, 90^{\circ}$ were analyzed, respectively. Moreover, in order to verify the advantage of structural optimization, analysis result of the original rectangular geometry with isotropic nylon was compared. $1 \mathrm{~mm}$ downward displacement of the indenter was applied on the top of the beam as shown Figure 6-11 (b). Figure 6-11 (a) shows the result of FEM analysis. The corresponding load for the displacement of each case was measured. The optimized structure reinforced by the optimal fiber orientation shows the highest stiffness response which was approximately as $132 \mathrm{~N} / \mathrm{mm}$. 9\% improvement was observed when compared with $0^{\circ}$ uniform tool-path. For other case, the structure with isotropic nylon shows $110 \mathrm{~N} / \mathrm{mm}$ of stiffness. These numerical results were verified by performing physical 3 points bending test. 


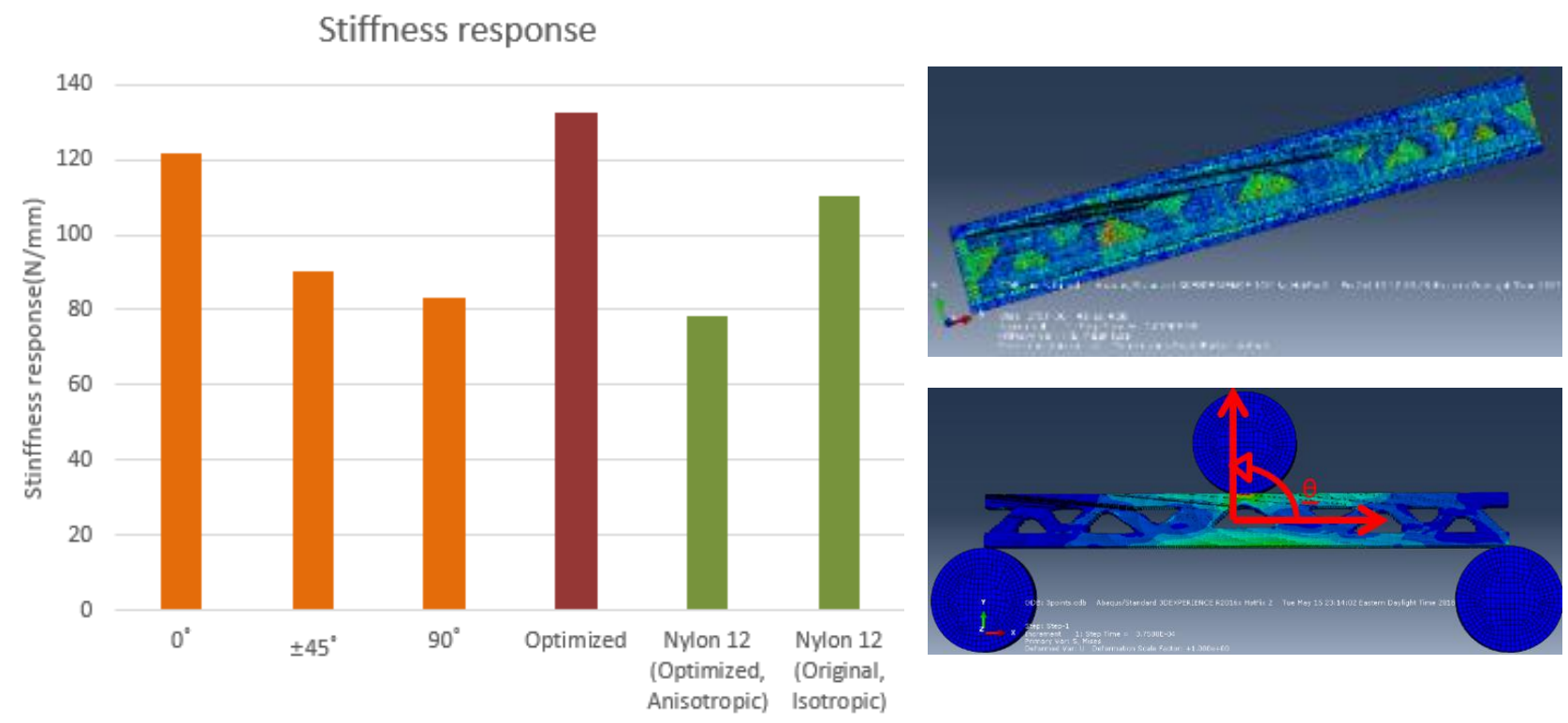

Figure 6-11 Comparison of maximum failure load between uniformly aligned and optimally distributed fiber reinforcement

\subsubsection{Manufacturing}

The optimized beam structure was fabricated as shown in Figure 6-12. The printing parameters employed were: nozzle diameter of $0.6 \mathrm{~mm}$, filament diameter of $2.75 \mathrm{~mm}$, nozzle extrusion temperature of $260^{\circ} \mathrm{C}$, build plate temperature of $110^{\circ} \mathrm{C}$, layer height of $0.2 \mathrm{~mm}$ and printing speed of $20 \mathrm{~mm} / \mathrm{s}$. Therefore, the total building time for one product was 5 hours.

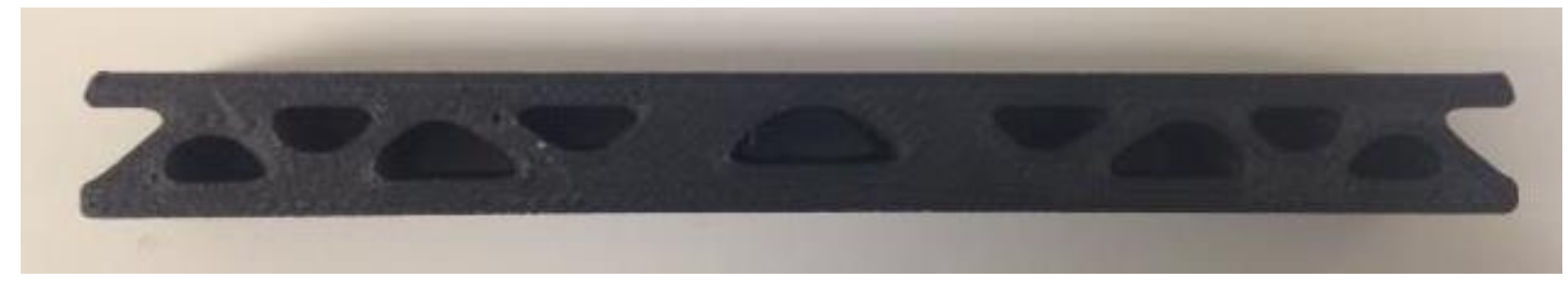

Figure 6-12 Optimized beam with carbon fiber reinforcement by FFF 3D printing

For the material of nozzle, Olsson ruby nozzle was used because abrasive carbon fibers may wear out the original nozzle materials such as bronze and steel. Figure 6-14 shows an example of nozzle damage and Olsson ruby nozzle. FFF based 3D printer Ultimaker 2+ was used to fabricate the structure as shown in Figure 6-13. In order to achieve better bonding with the plate and avoid a warping, yellow Kapton tape was applied to the build plate of Ultimaker $2+$. 


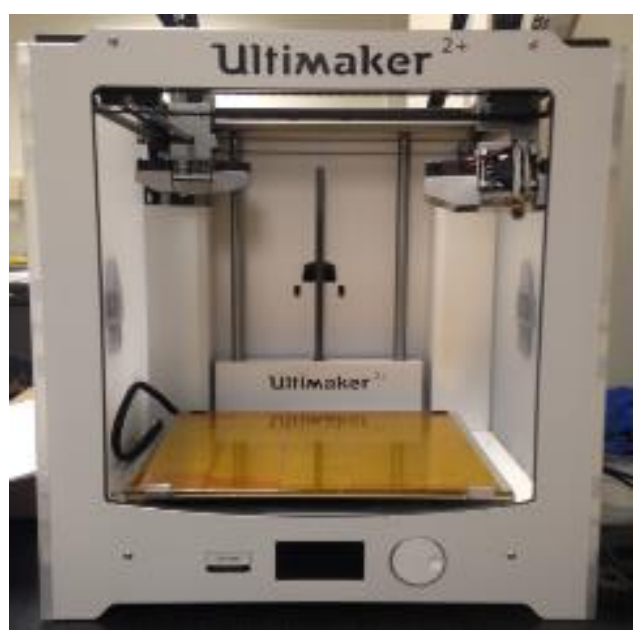

Figure 6-13 Upgraded Ultimaker 2+ in Dr. Kang's lab.
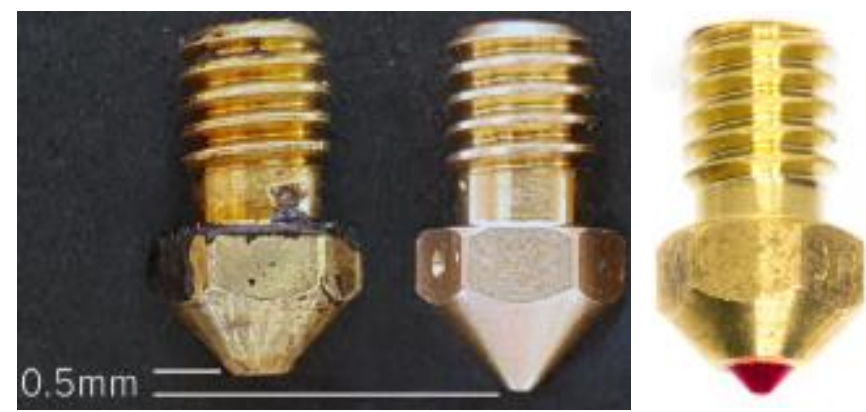

Figure 6-14 An example of nozzle damage and ruby nozzle.

\subsubsection{Three points bending test}
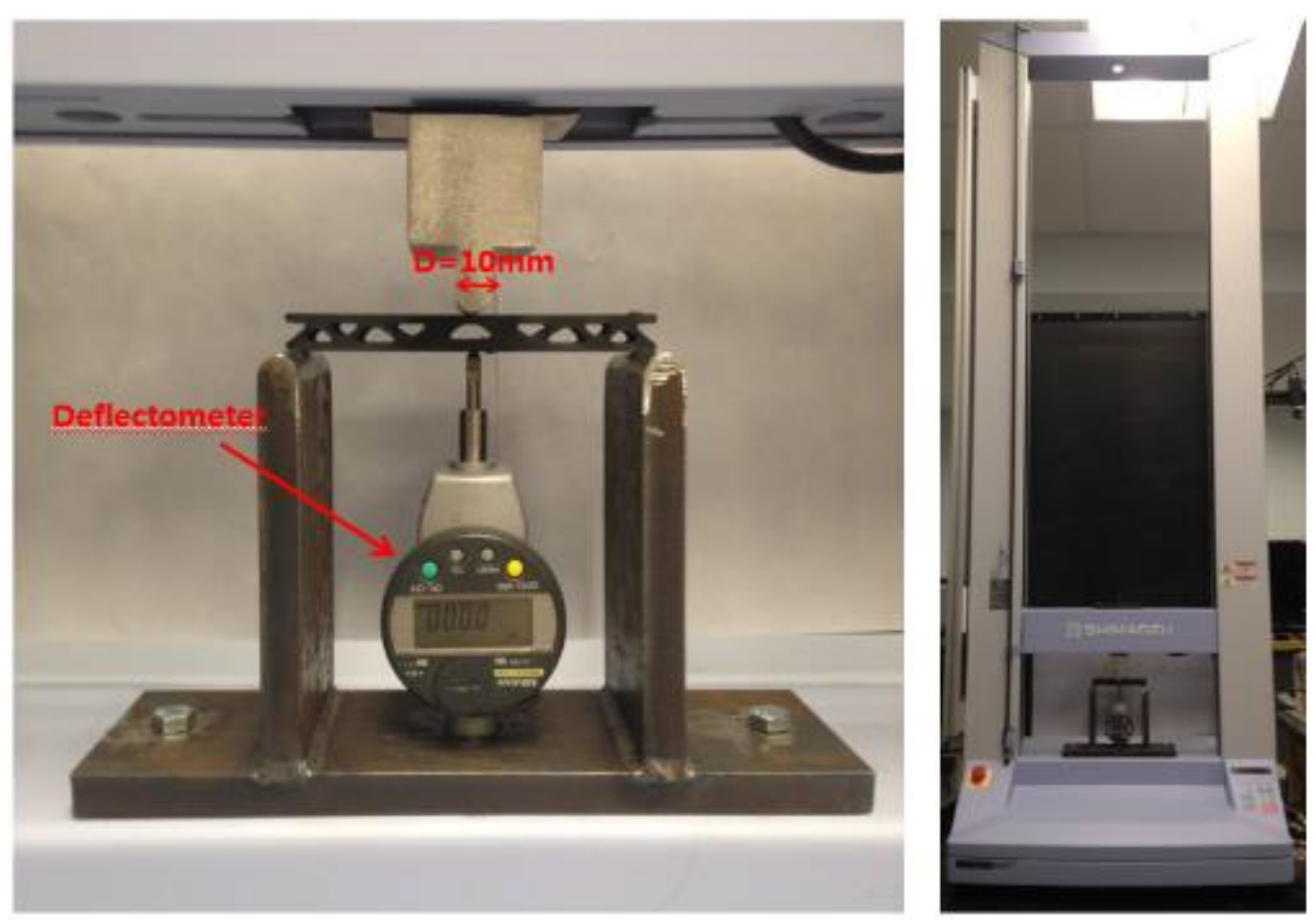

Figure 6-15 Three points bending test for the optimized beam

Three-point bending test is conducted to validate the FEA simulations of CFRP beam printed at optimized tool-path. For the comparison, beams uniformly printed at $45^{\circ}, 90^{\circ}$, and $0^{\circ}$ were tested as well to see the advantage of customized tool-path. universal mechanical test machine SHIMAZU AGS-X HC was used 
to measure load from the indenter. A digital diflectometer was installed on the bottom of the machine platform to measure the displacement at the middle of beam. The diameter of the load indenter was 10 $\mathrm{mm}$ and loading rate was $2 \mathrm{~mm} / \mathrm{min}$ determined by ASTM D790 using the equation $\mathrm{R}=\frac{Z L^{2}}{6 d}$. Parameters are as follows: $\mathrm{L}=$ support span, $\mathrm{d}=$ Depth of beam, and $\mathrm{Z}=$ Rate of straining of the outer fiber $(0.01 \mathrm{~mm} / \mathrm{mm} / \mathrm{min})$. Figure 6-15 shows three points bending test platform.

Figure 4.4 shows load vs displacement curve for each model. Three different test data were averaged. For the repeatability of the test results, three samples of each were tested and variation was less than $1 \%$. Detailed test results were attached in Appendix. The beam with the optimized tool-path shows the highest stiffness response and failure load $110 \mathrm{~N} / \mathrm{mm}$ and $192 \mathrm{~N}$, respectively. This is $7 \%$ and $10 \%$ improvement in stiffness response and failure strength when compared with beam printed at uniformly $0^{\circ}$

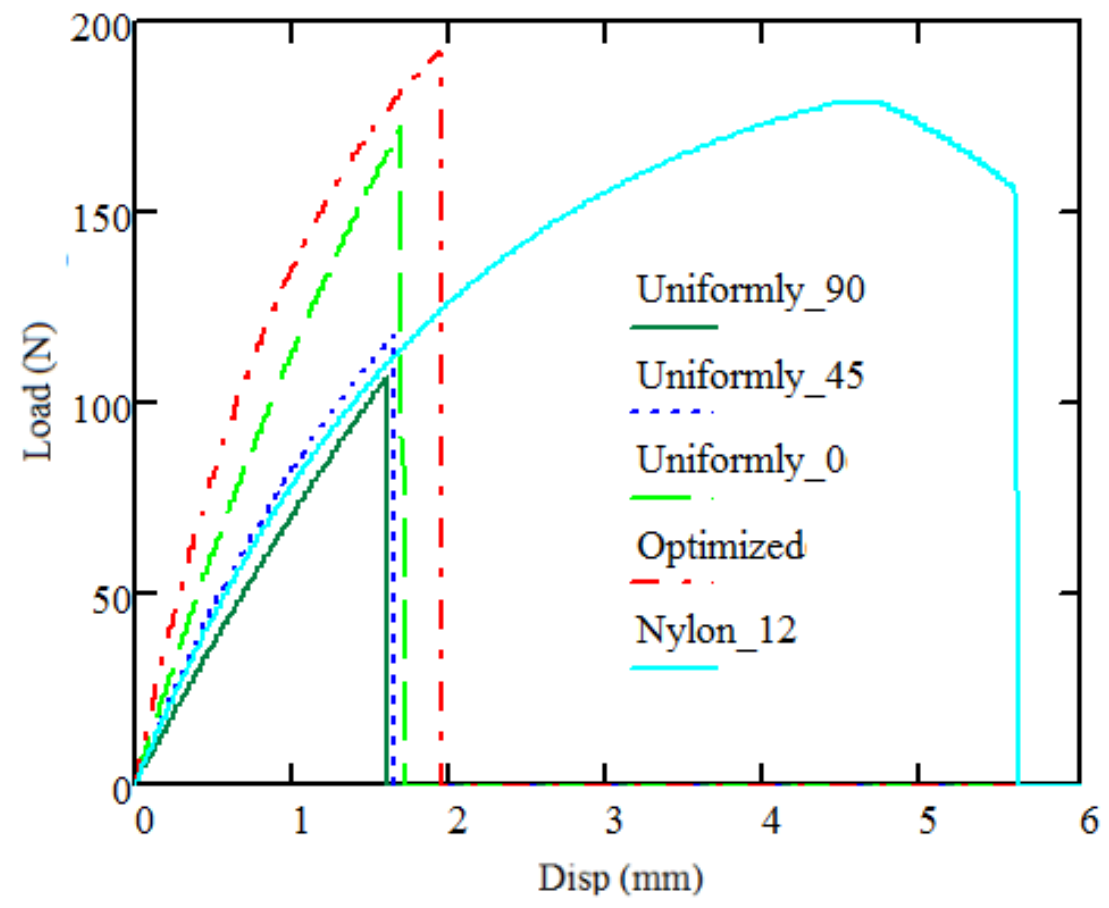

Figure 6-16 Comparison of load vs displacement curves of beams with different printing pattern

\subsubsection{Conclusions and Discussions}

Figure 6-17 shows the comparison of results between FEA simulation and experimental tests. Experimental tests exhibit approximately 15\% lower values compared with FEA simulation results, possibly due to manufacturing defects such as voids, missed alignment, or residual stress. For optimized beam, the difference of results shows about $17 \%$. This is because the customized tool-path has several 
cutting points, which means more frequently stop the extruding and move to different locations. cutting points could generate voids because of this mechanism.

\section{Three Points Bending Test}

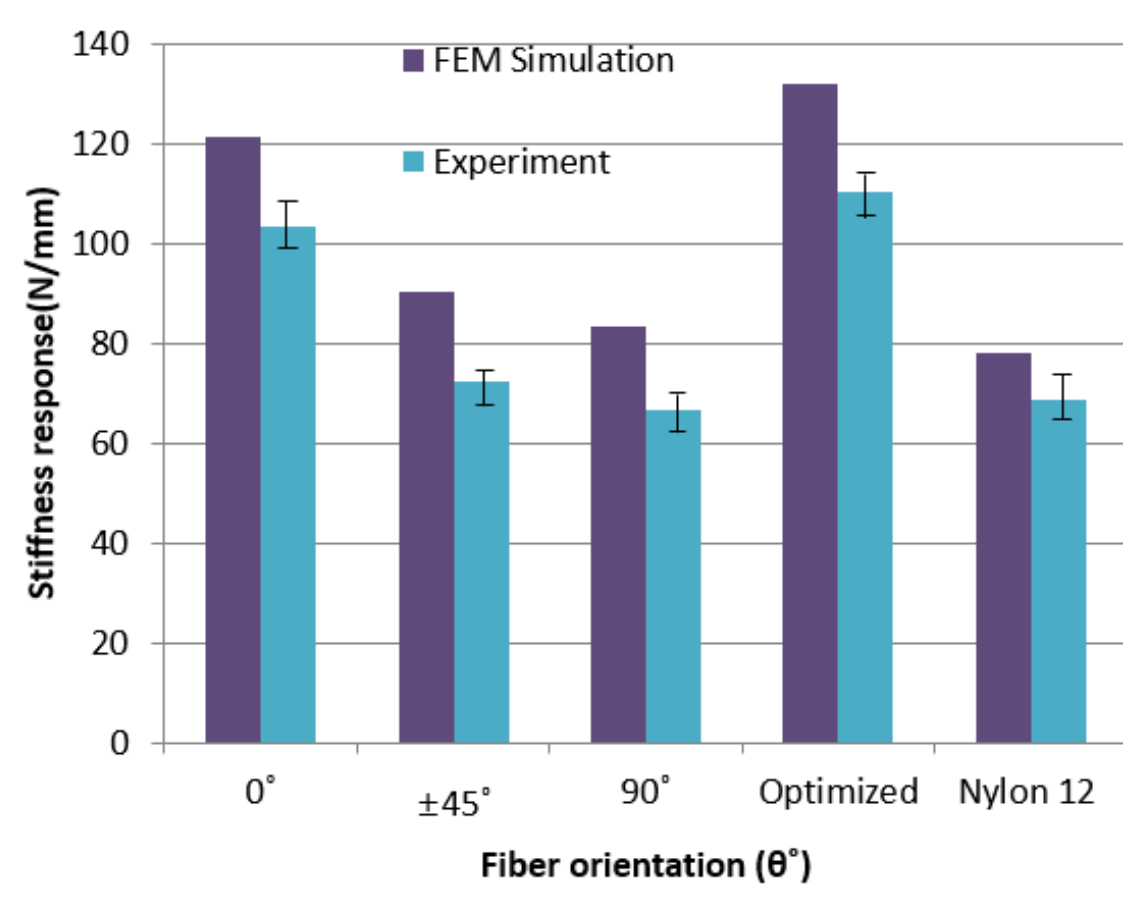

Figure 6-17 Comparison of results between FEA and Experimental tests

\subsection{Case Study II. - Stress concentration plate}

\subsubsection{Problem statement}
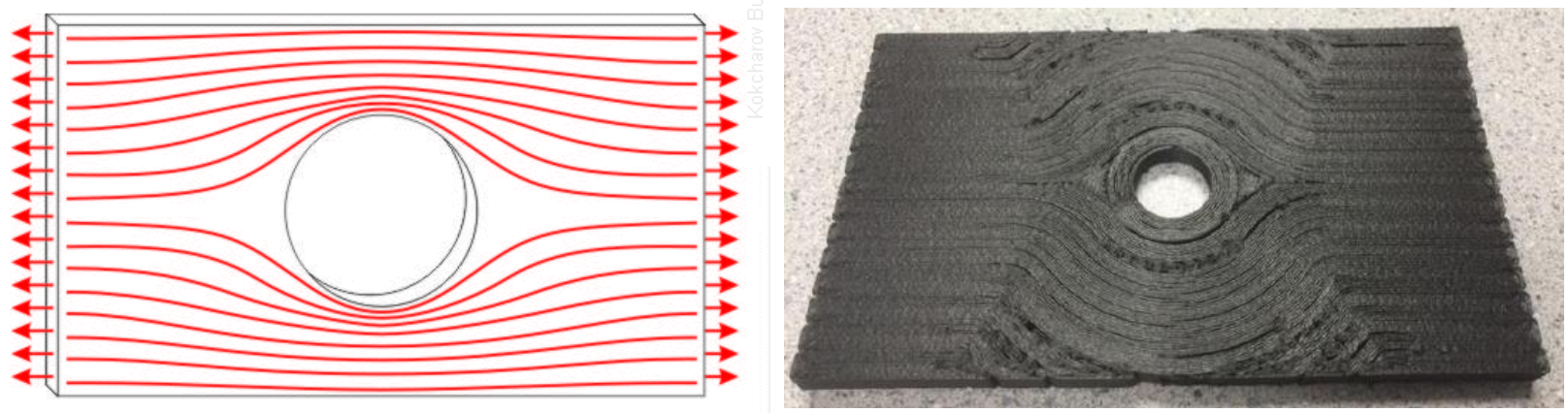

Figure 6-18 (a) Stress flow and (b) a FFF built CFRP sample of stress concentration plate

Stress concentration in plate with a hole was chosen to describe the advantage of the customized tool- 
path method. Mathematical analysis and experimental measurement show that in a loaded structural member, near changes in the section, distributions of stress occur in which the peak stress reaches much larger magnitudes than does the average stress over the section. This increase in peak stress near holes, grooves, notches, sharp corners, cracks, and other changes in section is called stress concentration. The section variation that causes the stress concentration is referred to as a stress raiser. Figure 6-18 shows stress flow and a CFRP sample of stress concentration plate.

\subsubsection{Finite element analysis}

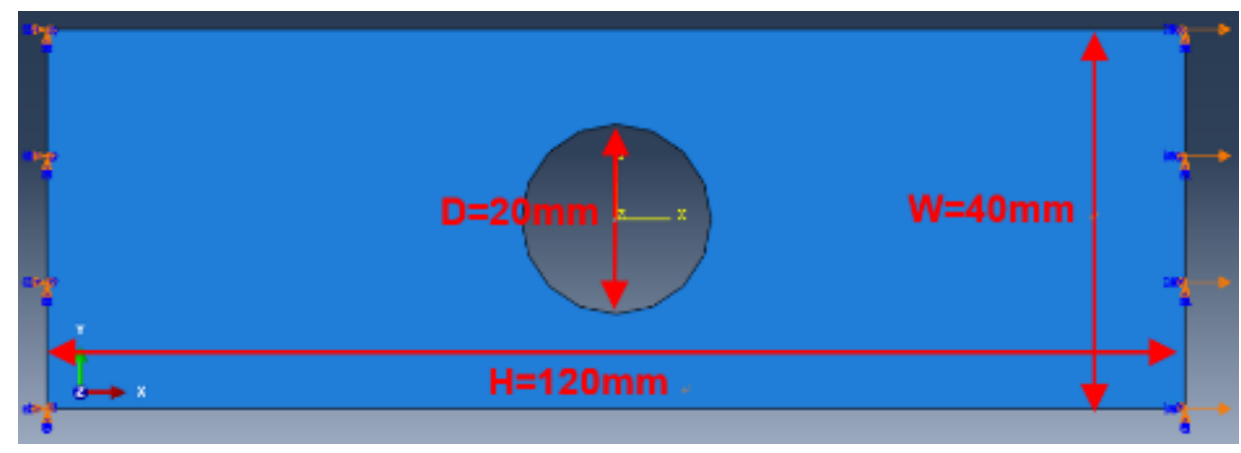

Figure 6-19 Stress concentration plate

In order to compute principle directions of each element, FEA stress analyses were carried out. In this work, ABAQUS ${ }^{\mathrm{TM}}$ was utilized. 2D shell element was applied for simplicity. As described previously, reticular elements were employed to connect elements in order to generate a tool-path. Height, width, and radius of the hole are $120 \mathrm{~mm}, 40 \mathrm{~mm}$, and $10 \mathrm{~mm}$, respectively. Figure 6-19 illustrates the example model.
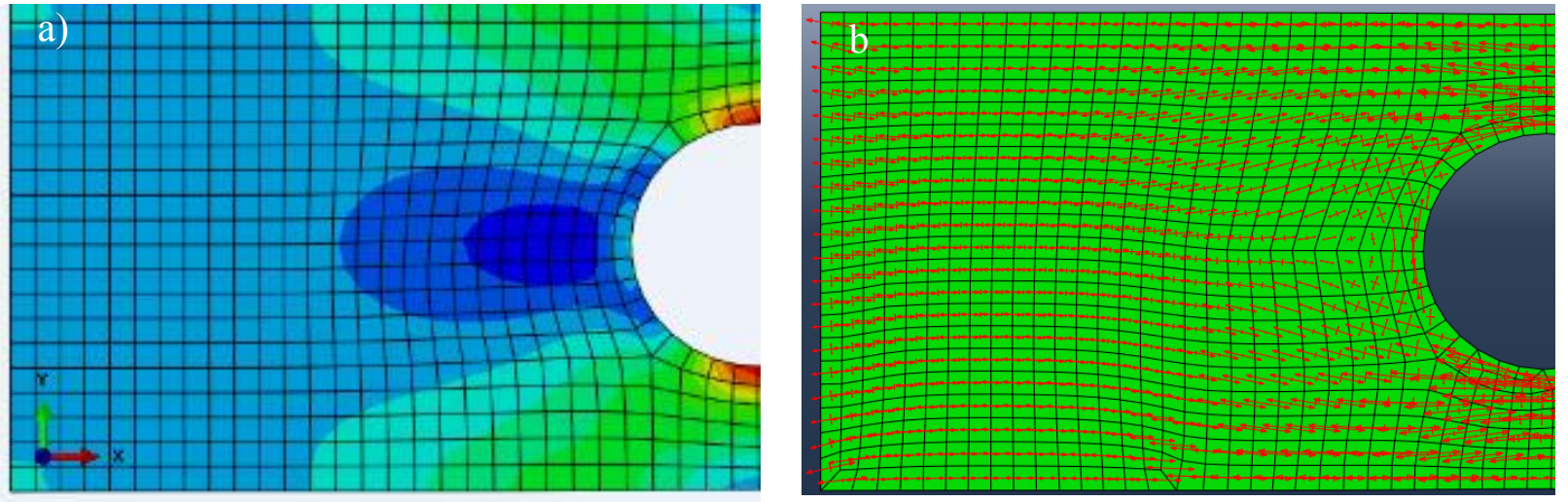

Figure 6-20 (a) stress field and (b) principal directions of stress concentration plate 
expected, high stress occurred around the center hole and their principal directions are aligned with the hole. Also, blue color in Figure 6-20 (a) indicates low stress region, in Figure 6-20 (b) shows this region as short length of arrow.

\subsubsection{Tool-path development}
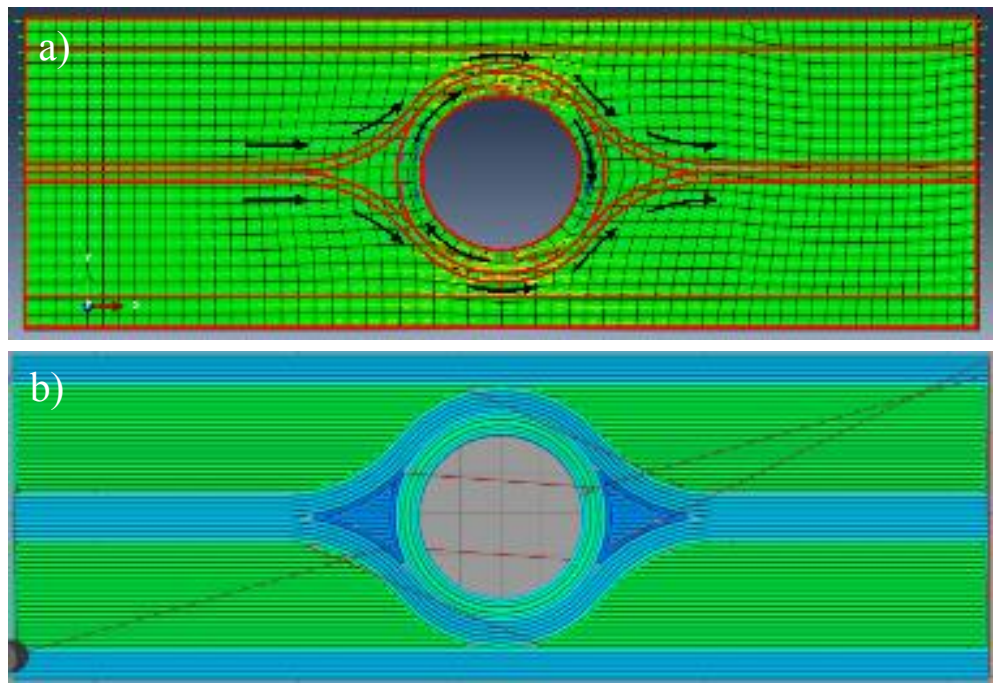

Figure 6-21 (a) Section division for tool-path and (b) complete optimized tool-path of stress concentration plate

Figure 6-21 shows the section division of the plate for tool-path using the proposed method. Each element is connected to create sections. Different tool-path is generated to each section based on principal directions of elements in sections. For example, the tool-path around the center hole is aligned with the circle as principal directions are parallel to the circle. Moreover, low stress region in Figure 6-20 (a) has $0^{\circ}$ uniform tool-path which is the same with tool-path in other major regions. Figure 6-21 (b) shows the complete tool-path development of the whole plate. 


\subsubsection{FE modeling}

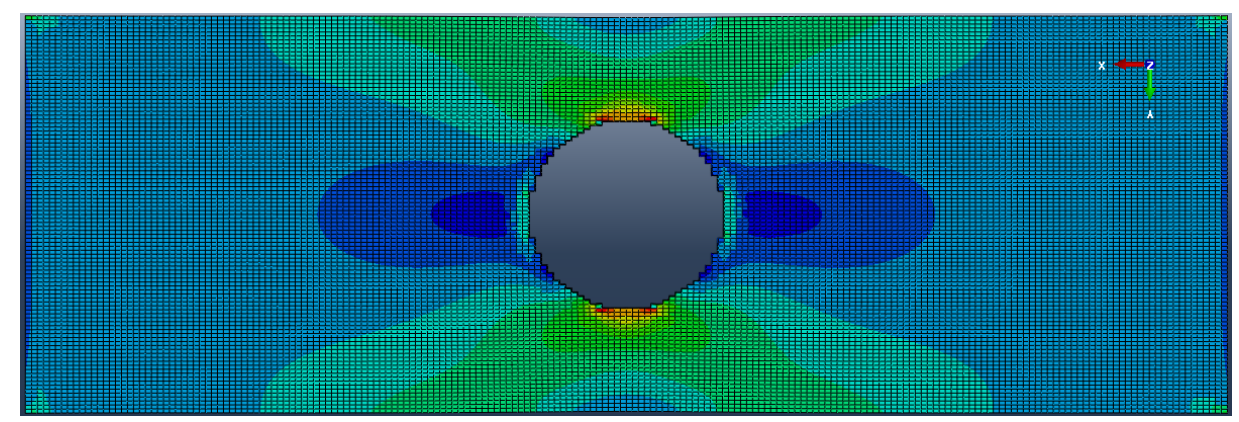

Figure 6-22 Stress distribution of the plate printed by the optimized tool-path

Figure 6-22 shows the stress distribution of the plate with the updated orthotropic material properties induced by the customized tool-path. High stresses still occurred around the hole, but relatively were more distributed in larger area compared with the plate with the initial isotropic material. 180 and 60 division number of $\mathrm{X}$ and $\mathrm{Y}$ coordinates were applied to generate square elements to reduce computation time. Total $180 * 60=10,800$ number of elements were generated. For the stress concentration plate printed by $0^{\circ}$ tool-path as shown in Figure 6-24 (a), shear strain reached the failure first, which is 0.00854 as tensile load increased. The corresponding tensile loading was about $3600 \mathrm{~N}$. The corresponding tensile strain was 0.0145 that is still in elastic range. On the other hand, for the plate printed by customized toolpath, under the same tensile loading $3600 \mathrm{~N}$, shear and tensile strain were 0.00765 and 0.0151 , respectively. $9 \%$ was lower in shear strain compared with $0^{\circ}$ case. This means printing aligned with the center circle take an advantage to resist shear stress around the hole. Figure 6-23 shows shear strain distributions of these two cases from FEA simulations.
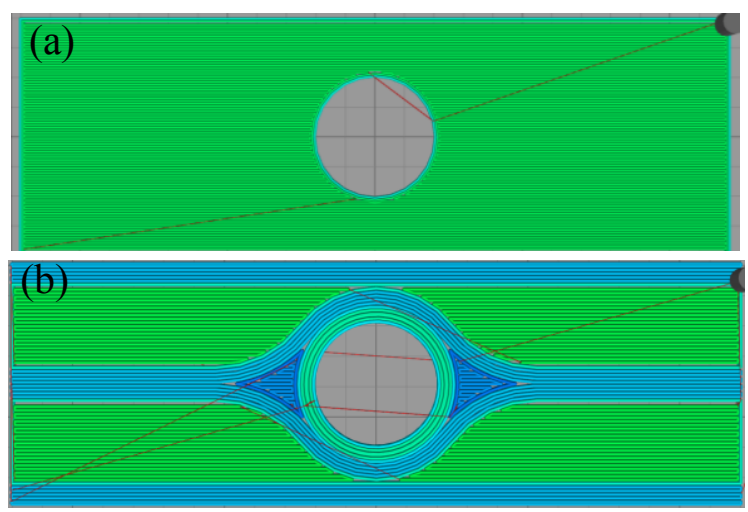

Figure 6-24 (a) $0^{\circ}$ uniform; (b) customized tool-path
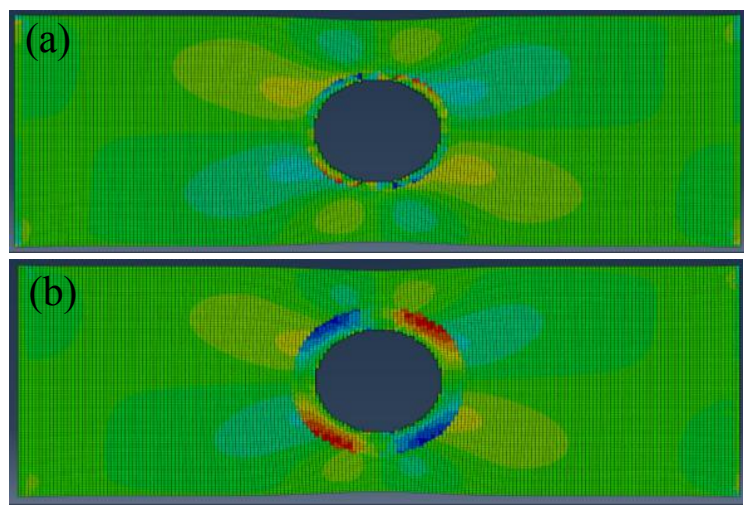

Figure 6-23 shear strain distribution of samples printed by (a) $0^{\circ}$ uniform; (b) customized tool-path 


\subsubsection{Tensile tests}
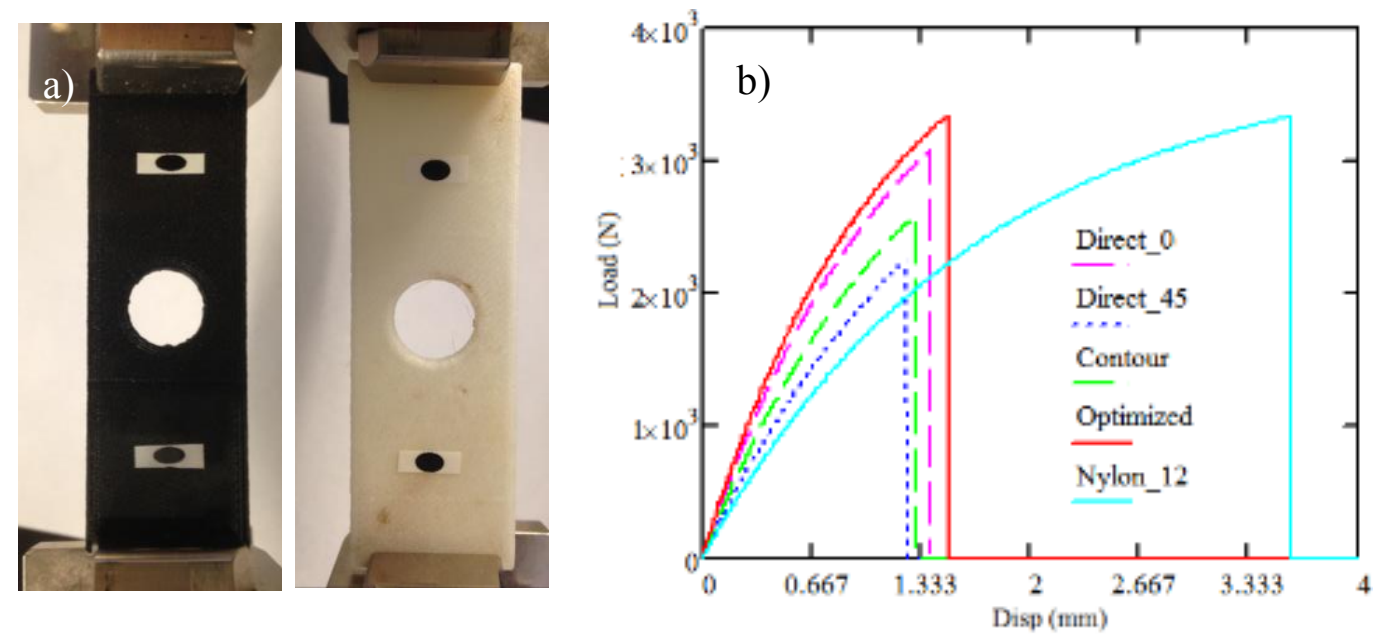

Figure 6-25 (a) tensile test and (b) test result of stress concentration plate

Relevant tensile test for stress concentration plates was performed to validate FEA simulation results. It was measured by a load cell attached to SHIMAZHU universal machine. Tracking sticker was attached to plates as an extensometer in order to measure displacements as shown in Figure 6-25 (a). Loading rate was $1 \mathrm{~mm} / \mathrm{min}$ and the data was recorded at every $0.01 \mathrm{sec}$. Using the force and displacement data, stiffness response was computed. Figure 6-25 (b) shows load vs displacement curves for each case. Averaged test results of each case are shown in Table 6-1.

Table 6-1 Tensile test results of stress concentration plate printed by various tool-path

\begin{tabular}{|c|c|c|c|}
\hline & $\begin{array}{ll}\text { Stiffness Response } \\
(\mathrm{N} / \mathrm{mm})\end{array}$ & $\begin{array}{l}\text { Failure Strength } \\
\text { (N) }\end{array}$ & $\begin{array}{l}\text { Max Disp } \\
(\mathrm{mm})\end{array}$ \\
\hline Direct-parallel $\left(0^{\circ}\right)$ & 2544.0 & 3087 & 1.39 \\
\hline Contour-parallel & 2161.7 & 2592 & 1.30 \\
\hline Direct-parallel $\left( \pm 45^{\circ}\right)$ & 1983.4 & 2262 & 1.25 \\
\hline Optimized & 2687.0 & 3349 & 1.50 \\
\hline Nylon 12 & 1690.0 & 3343 & 3.60 \\
\hline
\end{tabular}

The plate printed by the optimized tool-path shows the highest stiffness response and failure strength. For 
stiffness response, it is approximately $6 \%$ higher than direct-parallel $\left(0^{\circ}\right)$ case, $151 \%$ higher than the gear made by Nylon 12, respectively. For the failure strength, optimized plate shows $3349 \mathrm{~N}$ which shows $8 \%$ higher than direct-parallel $\left(0^{\circ}\right)$. It is noted that failure strength of optimized plate is almost the same with the plate made by Nylon 12 .

Figure 6-26 shows the comparison of stiffness response results between FEA simulations and physical tensile test. As similar with other case studies, approximately $5 \sim 10 \%$ difference in stiffness response showed up.

\section{Stress concentration plate test}

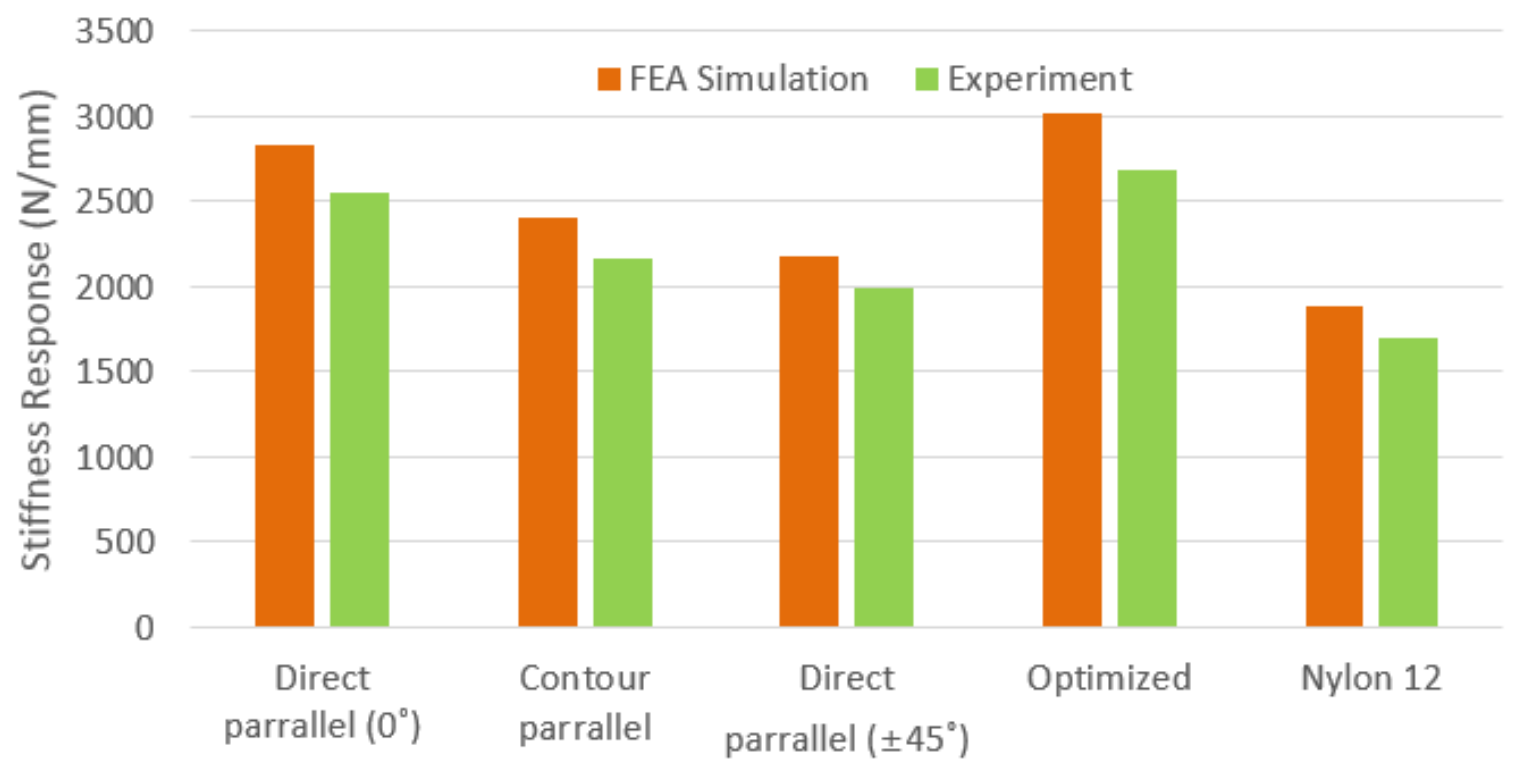

Figure 6-26 comparison of stiffness response between FEA simulations and physical experiments 


\subsection{Case Study III. - Spur Gear}

\subsubsection{Introduction}

Gears are mechanical components used for transmitting motion and torque from one shaft to another. Along with modern high speed manufacturing industry development, gears are now used widely in many applications ranging from automotive, robot and aerospace engines. Various types of gears are currently being manufactured for different industrial purposes. Spur gear is the most common type of gear. For the power or motion transmission, the tooth region generally experiences high stresses and are prone for failure and the hub region experiences less stresses. Figure 6-27 shows a failure of spur gear system in ball-milling machine in Dr. Kang's lab at WVU.
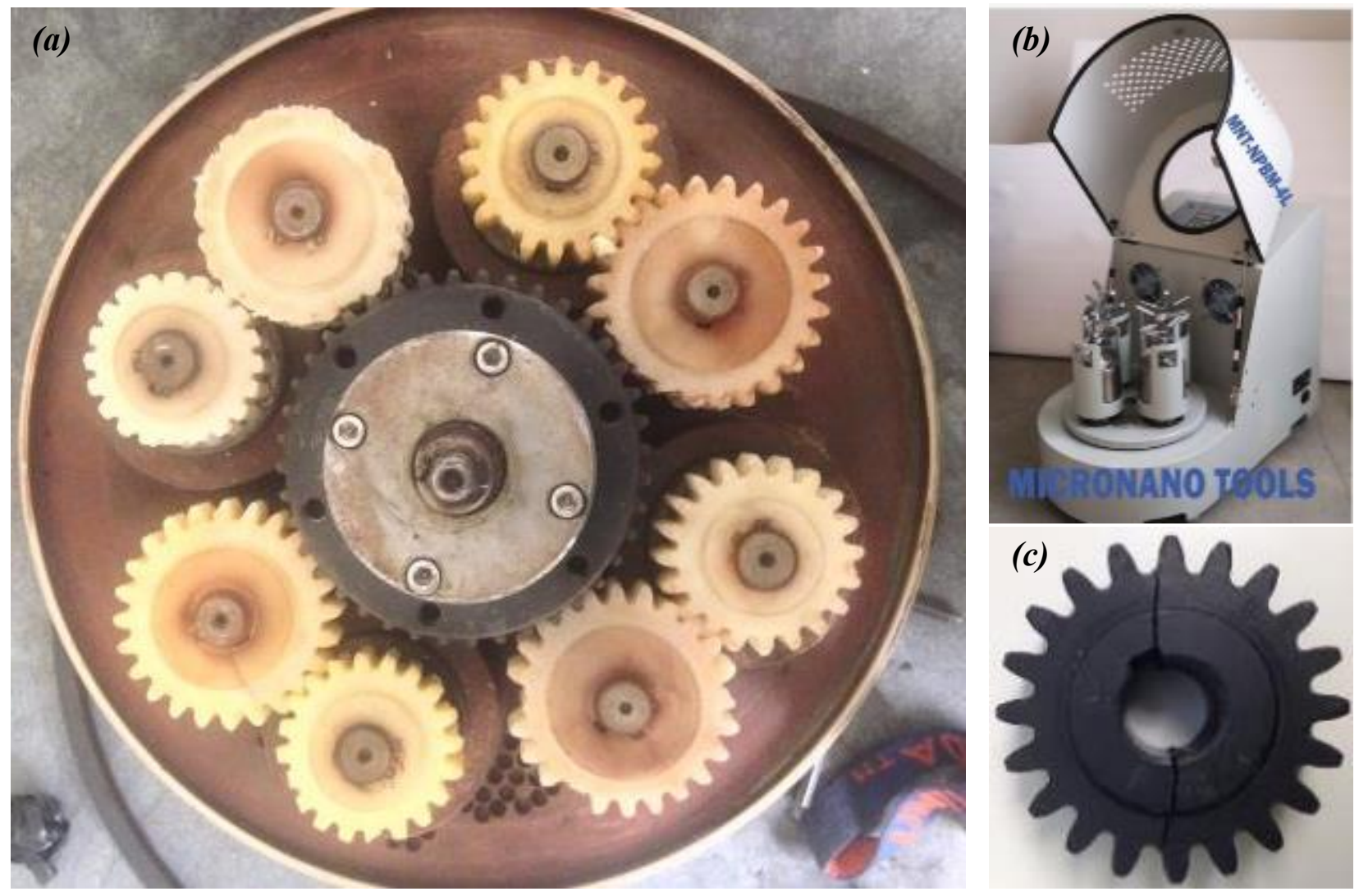

Figure 6-27 Spur gear damages of the ball-milling machine

In the Figure, gears were manufactured with nylon. The ball-milling machine was designed so that nylon 
spur gears are failed first to prevent a damage of the central main motor. The machine normally operates at $400 \mathrm{rpm}$, the tooth section of medium size gears experience high dynamic loading and fatigue. As shown in Figure 6-27(c) teeth were damaged, those were replaced with new set regularly. From the need for gears with improved durability, in this research a novel methodology of design and manufacturing for spur gears with carbon fiber reinforcement is introduced.

\subsubsection{Finite Element Analysis}

\subsubsection{Spur Gear Design}

In order to design the replacement spur gear, several critical geometries were measured. Since there is no information on parameters of the gear design in our ball-milling machine from the manufacturer, only outside diameter and number of teeth were measured and other parameters were computed using equations in Table 6-2. Figure 6-28 shows the required parameters for spur gear design.

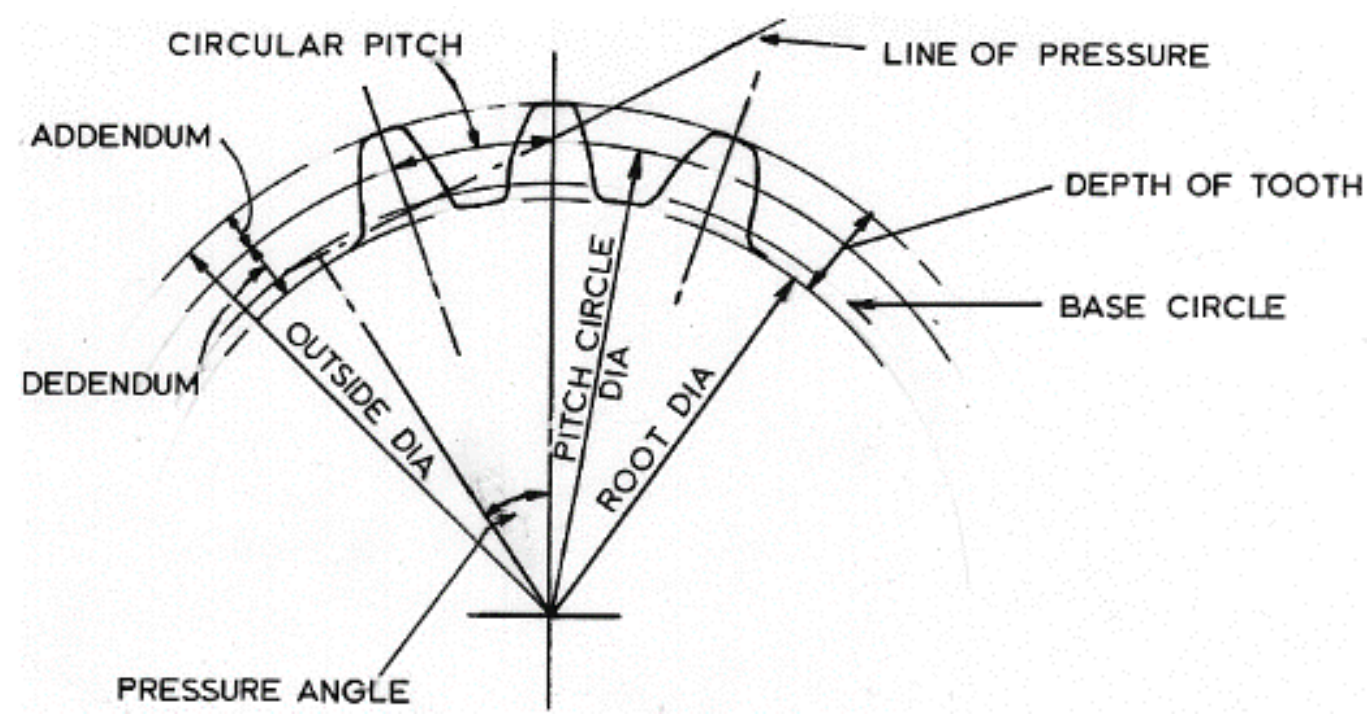

Figure 6-28 Parameters of spur gear design

Table 6-2 list of design parameters for spur gear

\begin{tabular}{lcr}
\hline Definition & Symbol & Values \\
\hline Number of teeth & $\mathrm{z}$ & 24 \\
Pressure angle $\left({ }^{\circ}\right)$ & $\mathrm{a}$ & 20
\end{tabular}


Module

Circular pitch (mm)

Pitch circle diameter (mm)

Outside diameter (mm)

Base circle diameter (mm)

Root diameter (mm) $\mathrm{m}$

3

$$
\mathrm{P}=\Pi^{*} \mathrm{~m}
$$

72.00

78.00

$\mathrm{d}_{\mathrm{o}}=\mathrm{d}+2 * \mathrm{~m}$

65.61

$\mathrm{db}=\mathrm{d}-(2+\Pi / \mathrm{z})^{*} \mathrm{~m}$

67.66

\subsubsection{Numerical analysis}

Numerical analysis was carried out to find high-stress regions and principal directions of individual elements in those regions. Two identical spur gears were imported from the AutoCAD product which was designed in the previous chapter. From the Figure 6-29, right shaft is treated as driven gear (gear) and left shaft is treated as drive gear (pinion). Since the material of the central shaft is metal and stiffness ratio of metal and nylon is approximately 100:1, it was assumed that right shaft is rigid and stationary throughout the operation and no displacement will occur in the shaft.

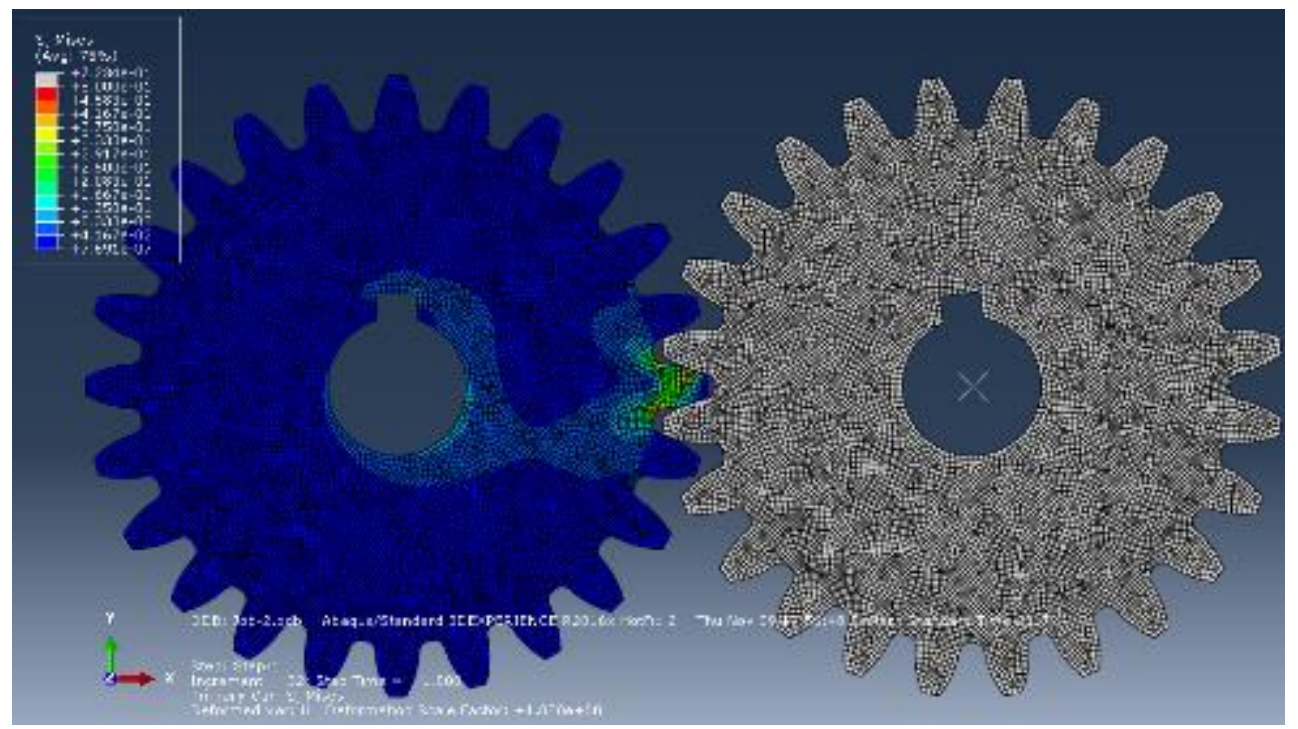

Figure 6-29 Stress analysis of spur gear

In Figure 6-29, right shaft with grey color indicates rigid. For the other shaft part, on the other hand, mechanical properties of Nylon 12 from Table 5-1 were applied. Young modulus was $1.7 \mathrm{GPa}$ and 
Poisson's ratio is 0.3 . Since the gear was additively manufactured layer by layer, 4-node CPS4R shell elements were applied for the simplicity. For the contact modeling between the driven gear (grey color) and drive gear, a general standard contact model was used. The friction in the contact surfaces of the gear teeth was disregarded. A torque of $10 \mathrm{~N}$ m was applied to the left shaft. Failure occurs due to the excessive bending stresses or contact stresses at the tooth root as shown Figure 6-30.

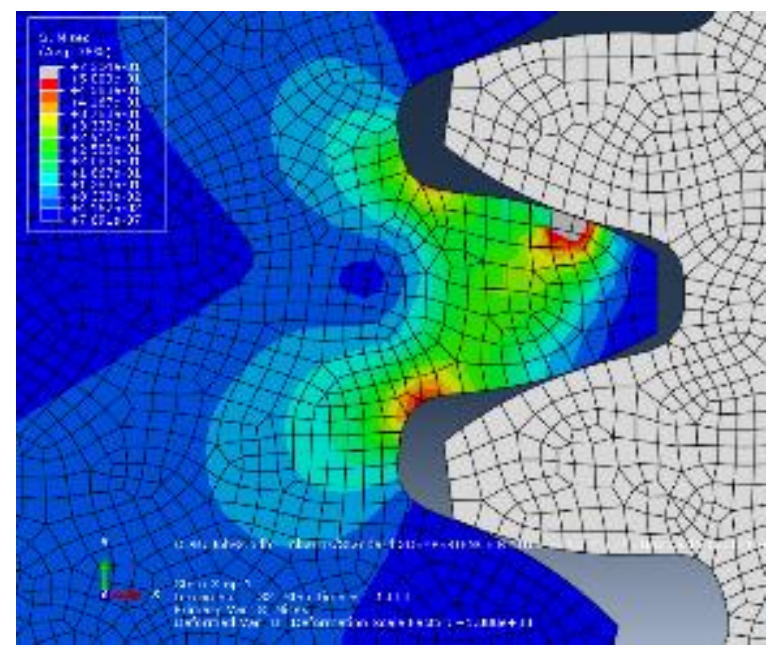

Figure 6-30 Maximum stress index for isotropic gear pairs

\subsubsection{Determination of Fiber Orientations}

Based on the information from the stress analysis, principal directions can be computed. Principal directions play a crucial role in the design of fiber orientation. Figure 6-31 shows principal directions of individual elements in tooth region(a) and center region(b), respectively.
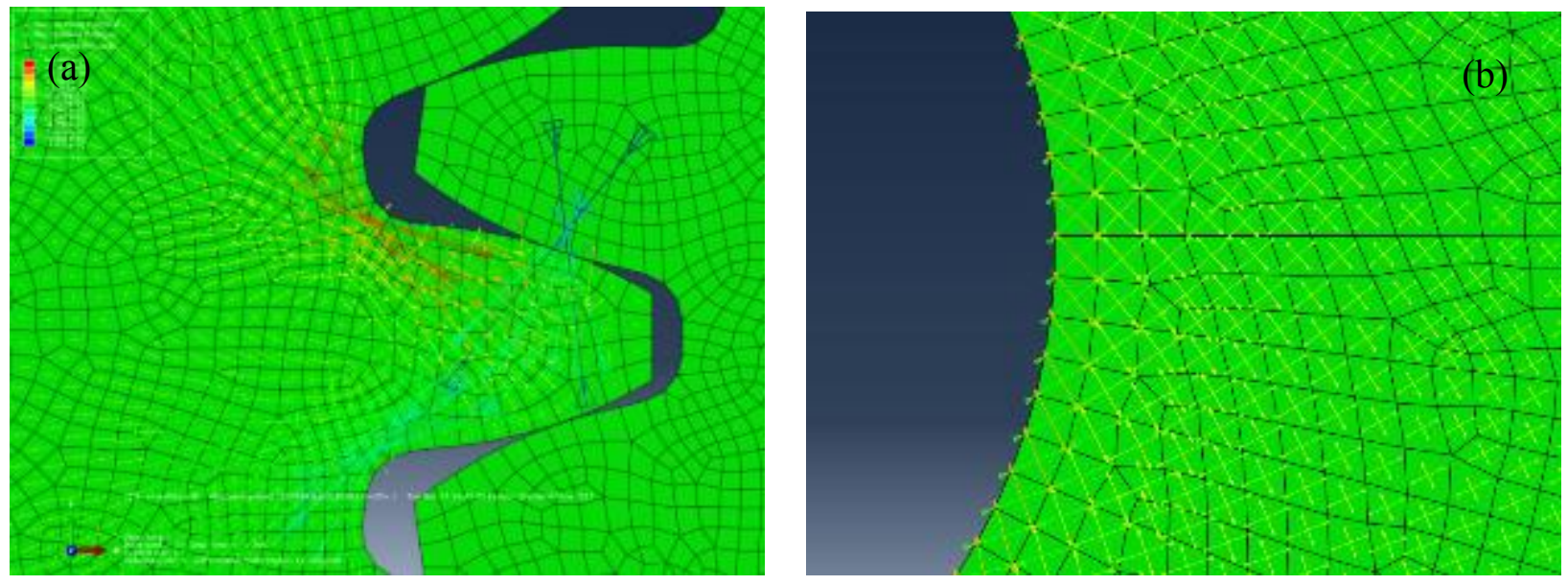

Figure 6-31 Principle directions of elements 


\subsubsection{Tool-Path Development - Spur Gear}

Using ABAQUS ${ }^{\mathrm{TM}}$, stress field was computed for the pre-described geometry under loading and boundary conditions. main gear which is made of steel was considered as rigid in the analysis. The isotropic material was applied to compute principal directions. The highest stress region showed up in tooth region.

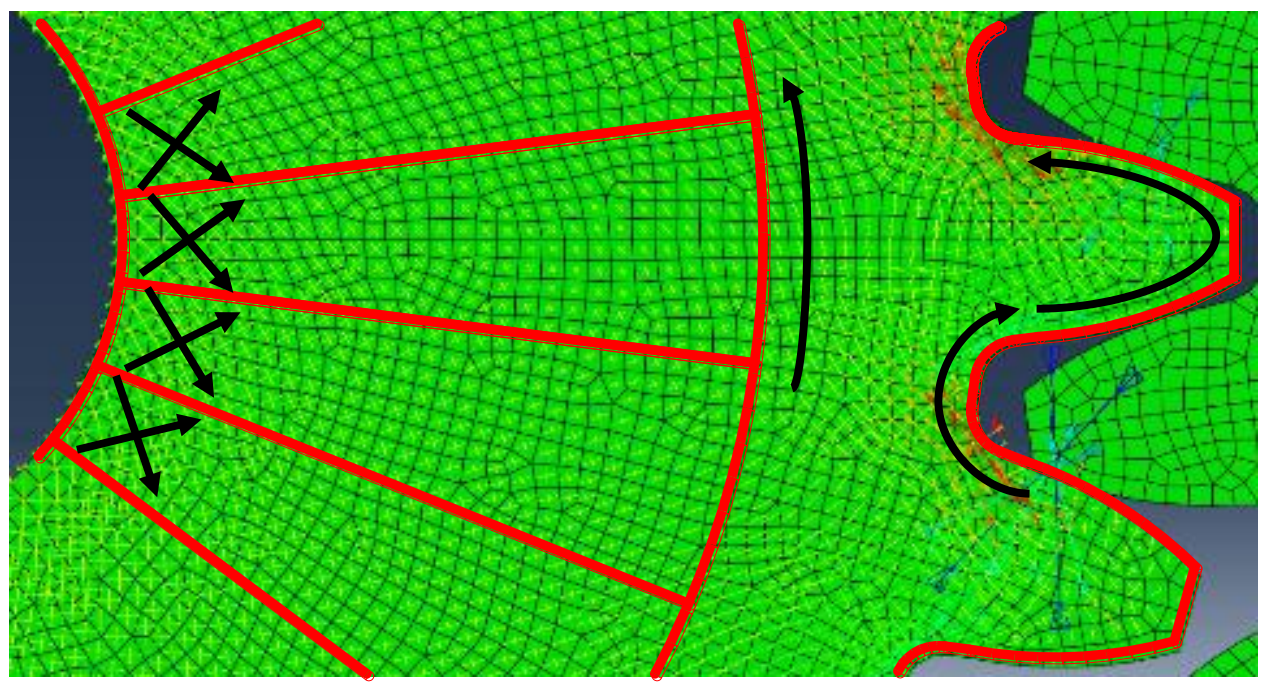

Figure 6-32 Section division of spur gear for tool-path

Figure 6-32 shows principal directions and section division for the printing. Different printing patterns were applied to individual sections. It is noted that principal directions of elements in tooth region are oriented along the gear tooth profile. Theoretically, ideal way to reinforce the gear is to align fibers with the tooth profile in the high-stress region. Figure 6-33 shows the optimized tool-path for the gear.

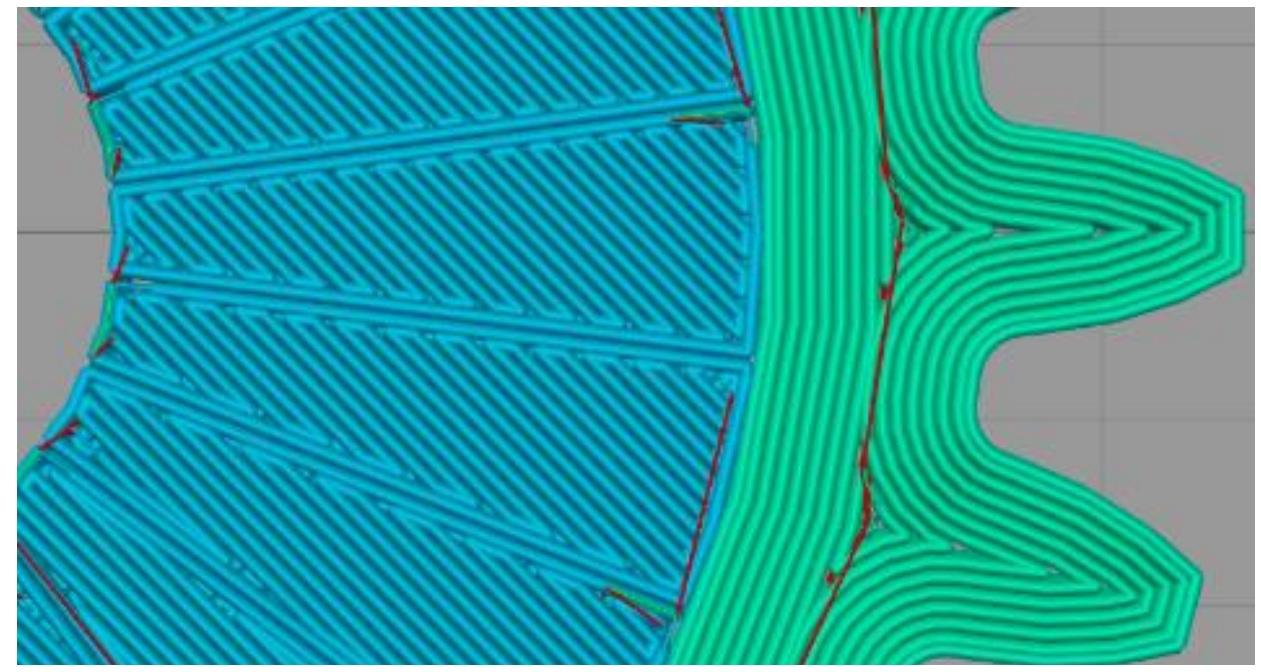

Figure 6-33 Optimized tool-path for spur gear 


\subsubsection{FE Modeling - Spur Gear}
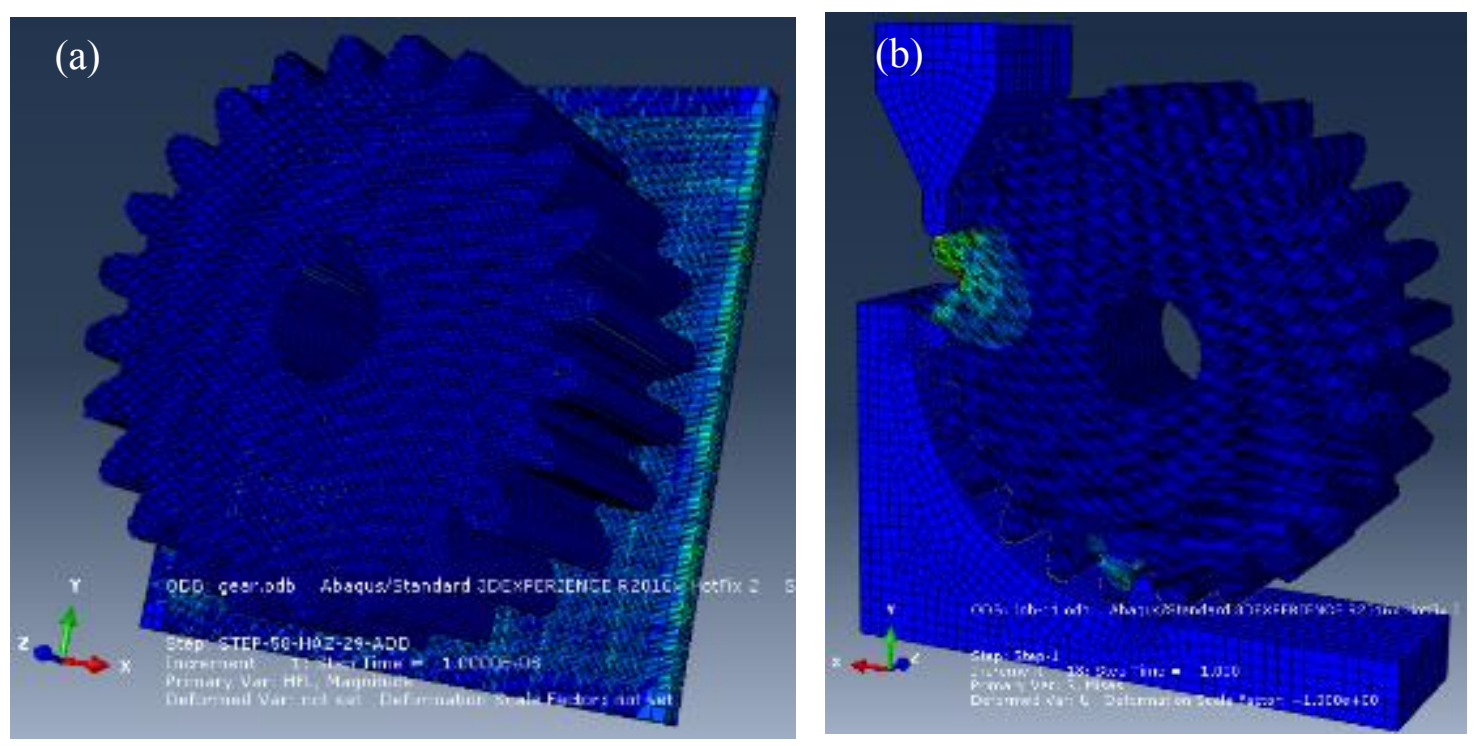

Figure 6-34 FE modeling (a) and compression test simulation (b)

In order to apply orthotropic material properties caused by customized tool-path. X,Y coordinate information from G-codes was utilized. Division numbers for X and Y coordinates were 150, respectively and only initial 8 layers were considered to reduce computation time. Total $150 * 150 * 180,000$ was used for gear modeling. After the gear was modeled, compression test platform was set up to see the advantage of the optimized tool path as shown in Figure 6-34 (b). Principal stresses of gears with the optimal fiber orientations, $\left[ \pm 45^{\circ}\right] \mathrm{s}$ and unidirectional orientation with $\left[0^{\circ}\right]$ were analyzed to compare the structural performance, respectively. Gears made by Nylon 12 was tested as well to see the advantage of CFRP material. Vertical displacement $1.0 \mathrm{~mm}$ was applied and high stresses were generated at tooth root region. A PC with a $2.4 \mathrm{GHz}$ Core CPU and 8GB RAM was used. Total computation time was approximately 23 hours. 


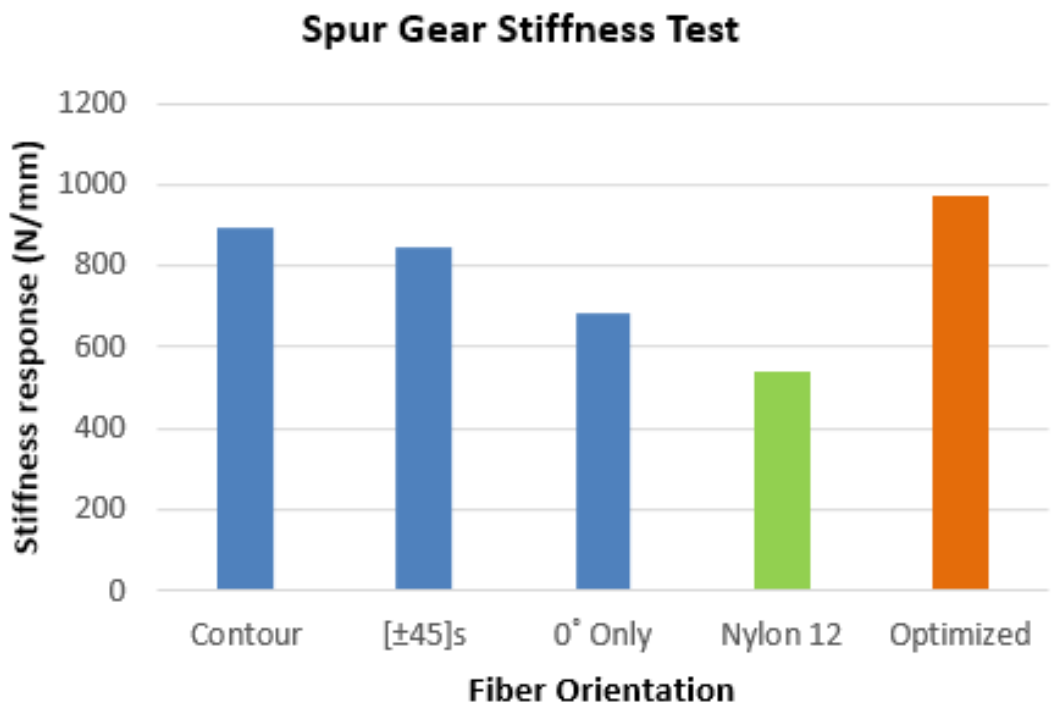

Figure 6-35 Spur gear stiffness test results

Figure 6-35 shows a comparison of FEM results. The principal stresses at the critical location of each case were measured and stiffness response was computed. The gear with the optimal fiber orientation shows the highest stiffness response of $981 \mathrm{~N} / \mathrm{mm}$. For other cases, contour-parallel, $\left[ \pm 45^{\circ}\right] \mathrm{s},\left[0^{\circ}\right] 8$, and Nylon 12 showed 894N/mm, 847 N/mm, 682 N/mm, and 539 N/mm. respectively. Approximately 9\% and $82 \%$ improvement of in stiffness was observed when compared with contour-parallel tool-path and Nylon 12 respectively.

\subsubsection{Compression Test - Spur Gear}
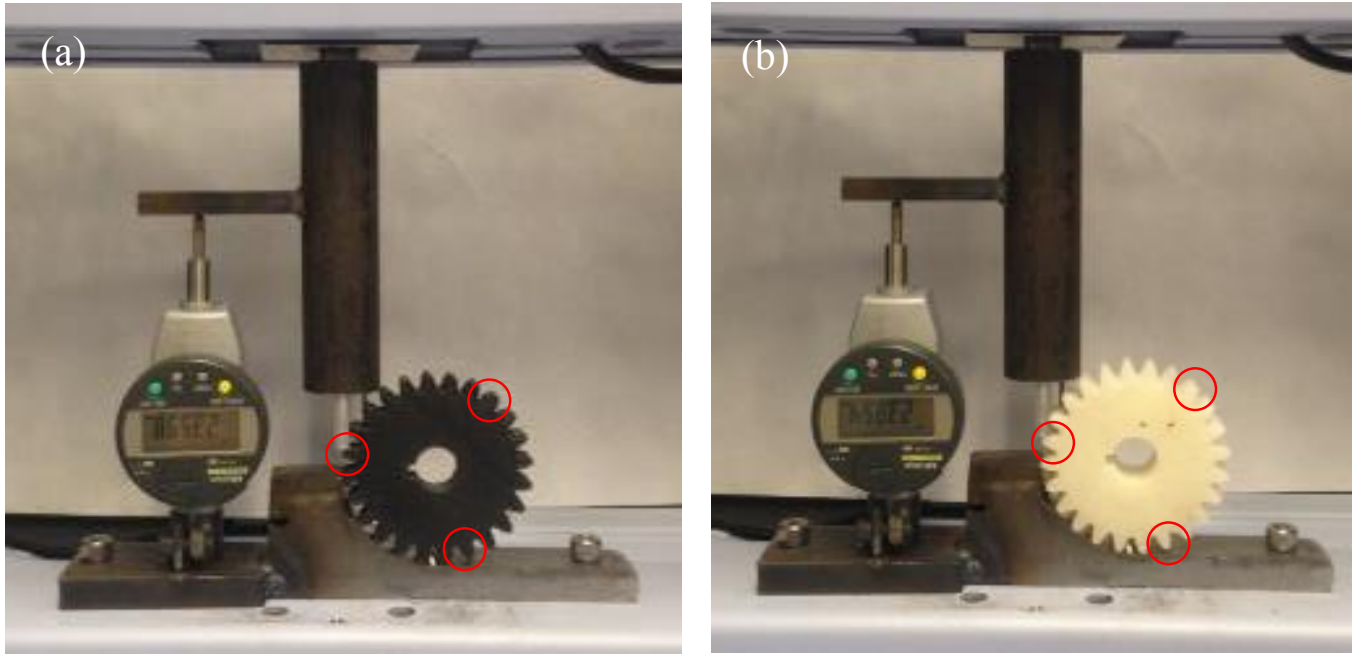

Figure 6-36 Spur gear stiffness test for CFRP (a) and Nylon 12 (b) 
In order to validate the FEA simulation results, relevant compression test for spur gear was performed. As previously discussed, high stress was generated in teeth region. Therefore, compressive force is applied to the tooth region as shown in Figure 6-36. It was measured by a load cell attached to SHIMAZHU universal machine. The corresponding displacement was measured by a digital deflectometer which has $0.0001 \mathrm{~mm}$ accuracy. Loading rate is $1 \mathrm{~mm} / \mathrm{min}$ and the data was recorded at every $10 \mathrm{sec}$. For the repeatability, tooth in three different locations were tested as indicated in Figure 6-36. Using the force and displacement data, stiffness response was computed.

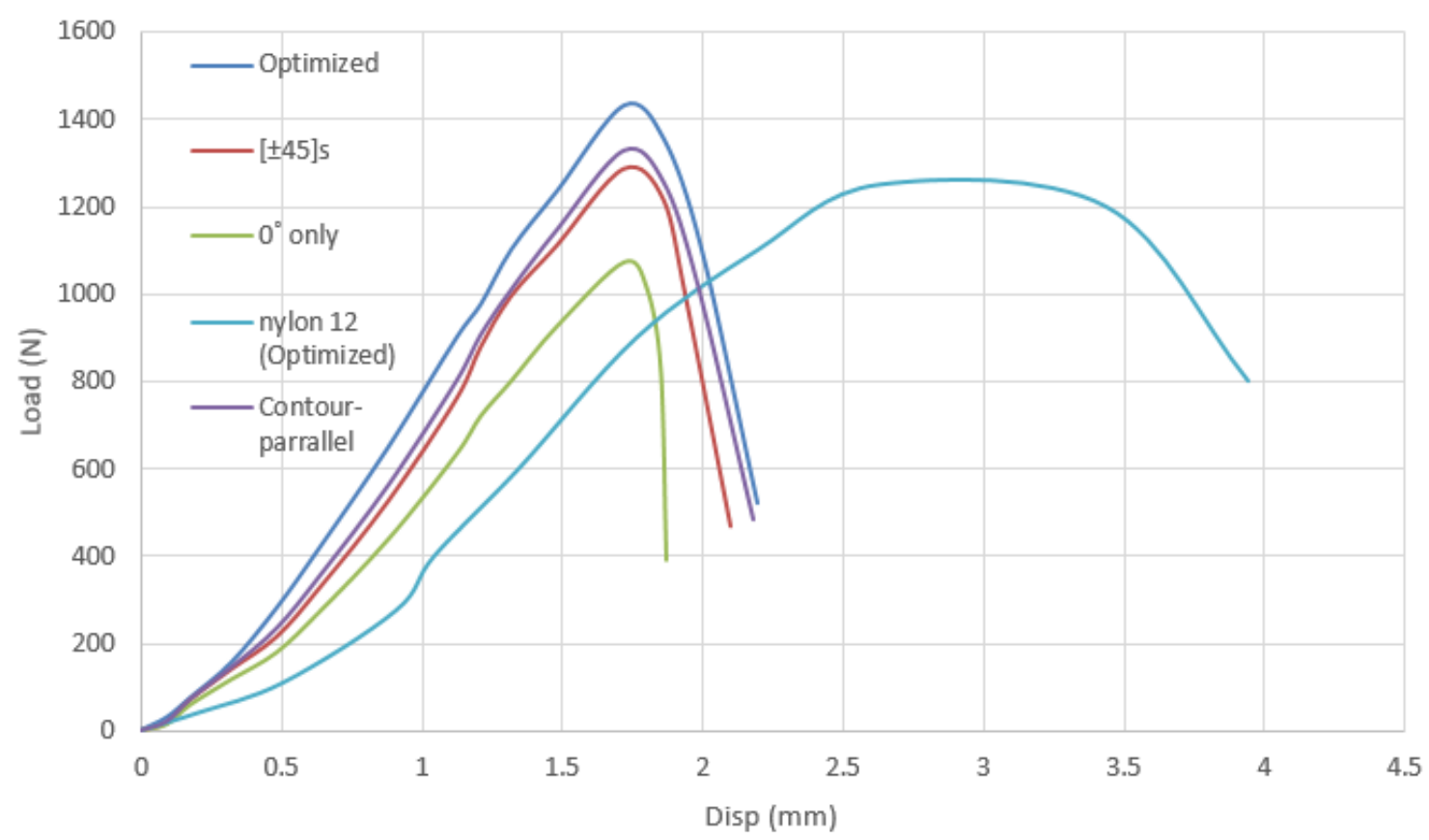

Figure 6-37 Load vs Displacement curves of CFRP spur printed by various tool-path

Figure 6-37 shows load vs displacement curves for each case. Averaged test results at three different locations are shown in Table 2.4. For the repeatability of the test results, three different locations of each were tested and variation was less than 1\%. Detailed test results were attached in Appendix.

Table 6-3 test results of stiffness response of CFRP and Nylon 12

\begin{tabular}{lrrrr}
\hline & $\begin{array}{c}\text { Stiffness Response } \\
(\mathrm{N} / \mathrm{mm})\end{array}$ & $\begin{array}{c}\text { Failure Strength } \\
(\mathrm{N})\end{array}$ & \multicolumn{2}{c}{$\begin{array}{c}\text { Max Disp } \\
(\mathrm{mm})\end{array}$} \\
\hline Contour & 778.6 & 1330 & 2.18 \\
Optimized & 834.6 & 1430 & 2.19
\end{tabular}




\begin{tabular}{llll}
{$[ \pm 45] \mathrm{s}$} & 754.6 & 1287 & 2.10 \\
$0^{\circ}$ only & 607.0 & 1073 & 1.87 \\
Nylon 12 & 475.1 & 1250 & 3.94 \\
\hline
\end{tabular}

The gear printed by the optimized tool-path shows the highest stiffness response and failure strength. It shows approximately $7 \%$ higher in stiffness response than the gear printed at contour-parallel and $175 \%$ higher than the gear made by Nylon 12, respectively. For the failure strength, optimized gear shows 1430 $\mathrm{N}$ which shows $8 \%$ higher than contour-parallel. It is noted that those made by CFRP shows brittle behavior. Once the internal stress reaches the failure level, teeth was taken out immediately. For Nylon 12 however, it was plastically deformed after yield stress, doesn't show fracture failure. Figure 6-38 illustrates the deformed gear.

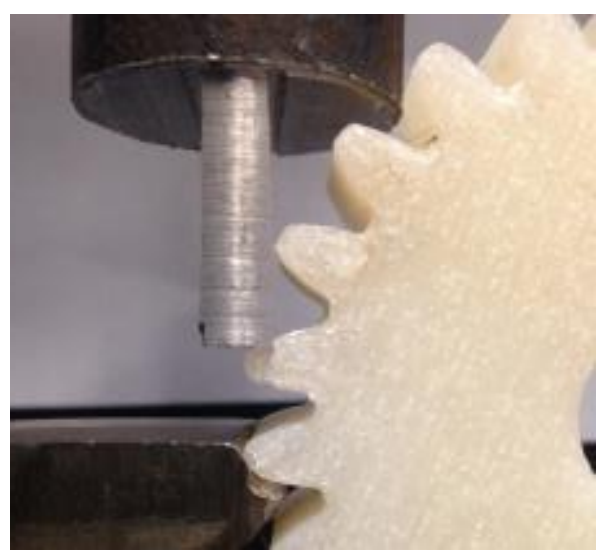

Figure 6-38 Deformed teeth of Nylon 12 spur gear

\subsubsection{Conclusions and discussions}

Figure 3.4 shows the comparison of stiffness response results between FEA simulation and physical experiment. Approximately 10\% difference in stiffness response showed up between FEA results and experimental tests. This is because of manufacturing defect such as voids, miss alignment, and residual stress. 


\section{Spur Gear Stiffness Test}

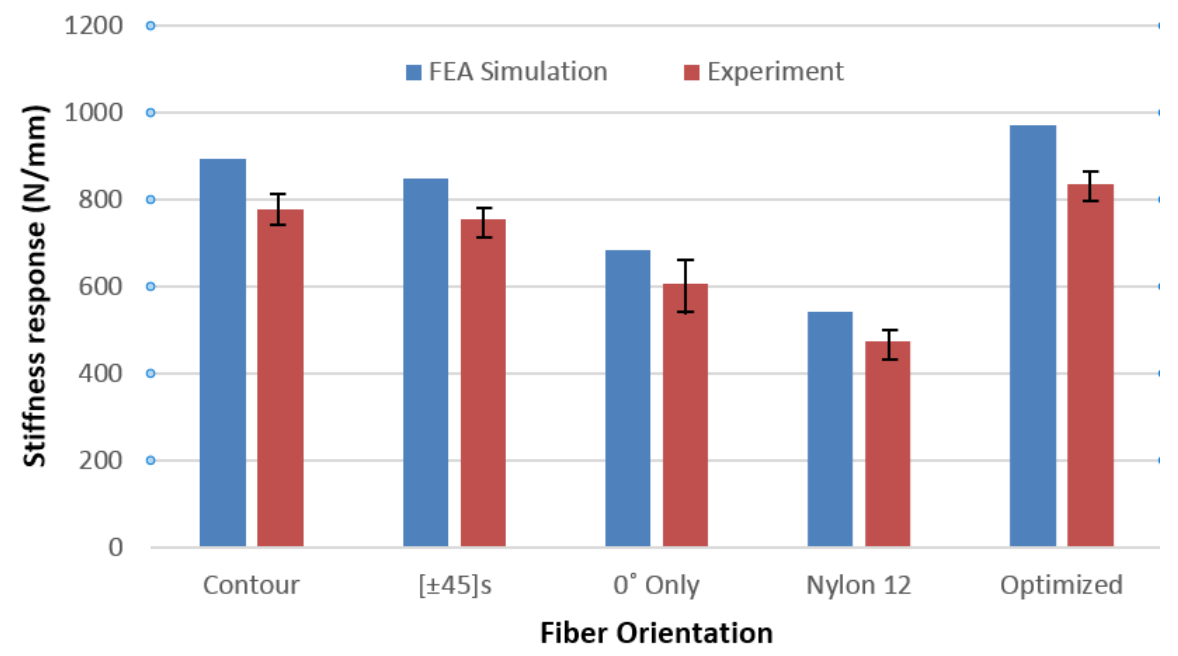

Figure 6-39 Comparison of results between FEA simulation and experimental tests

\subsubsection{Part Application for Ball-Milling Machine.}
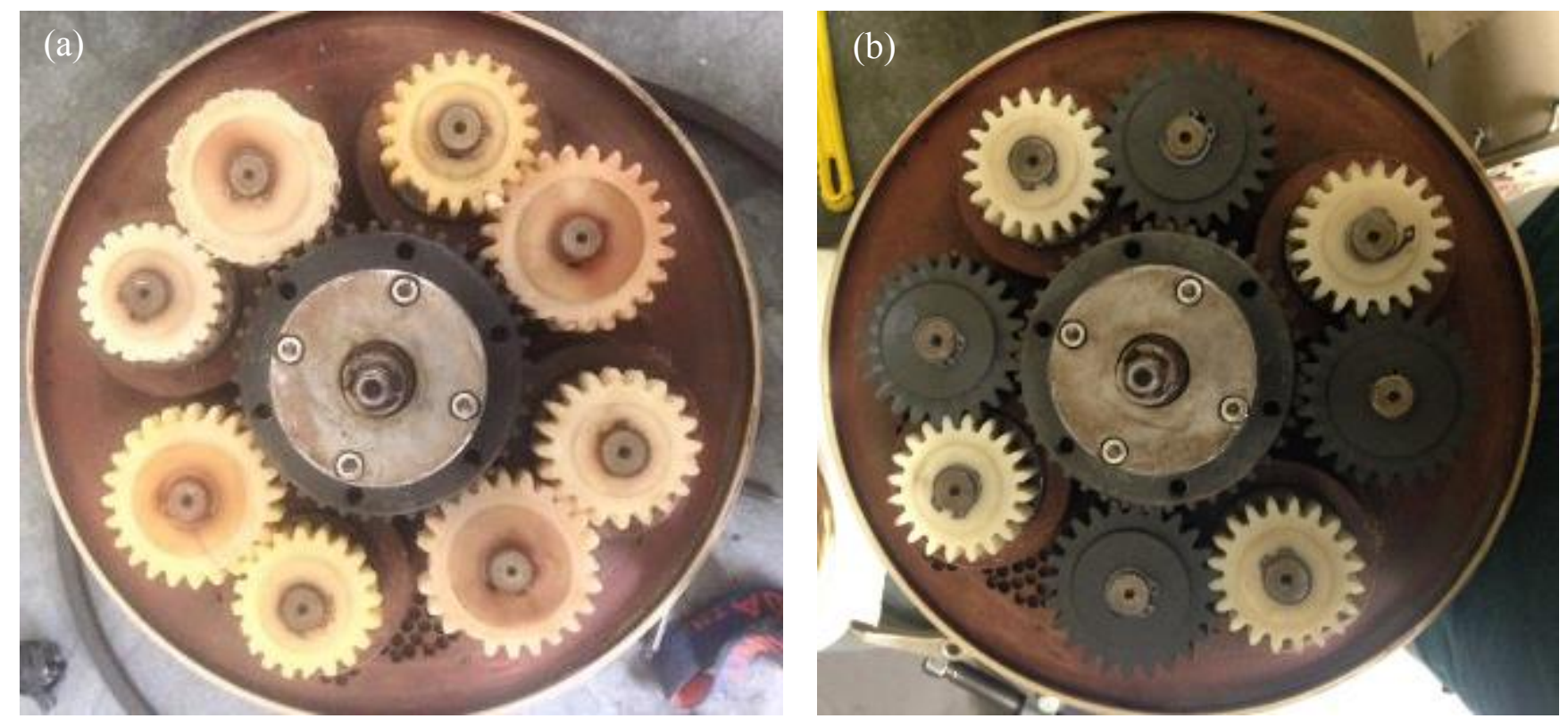

Figure 6-40 Gear set of ball milling machine, (a) damaged original gears (b) replacement of CFRP gears

To validate structural performance of the 3D printed gear, original nylon spur gear parts of ball-milling machine was replaced with CFRP 3D printed gears as shown in Figure 6-40(b). Since the maximum dynamic force was generated from the main motor gear at the center of machine, only medium size gears were replaced to have them directly experience the maximum force. The dynamic force is approximately $530 \mathrm{~N}$. Considering the maximum yield strength from the compression test, the safety ratio is about 2.7. 
The detailed calculation was attached in Appendix. The machine operated at $400 \mathrm{rpm}$, one cycle was 40 hours.

Figure 6-41 shows the failure of gears from the first trial. After 17 cycles (680 hours), one of the gears was failed. Failure mechanism was investigated. If the gear slightly tilts during the operation shear and tensile stress are generated. As shown in Table 5-1, CFRP parts are prone to shear or tensile stress between layers because of inherent nature of layer by layer manufacturing. Once it reaches the failure stress, parts of the gear are catastrophically taken out, not deformed since it is brittle. That was the reason for that ball-milling doesn't show any signals of failure such as abnormal noise. If one of jars doesn't spin during the operation, the only thing to recognize the failure was debris on the floor under the machine. Relatively low shear and bonding strength and brittleness are obviously disadvantages of CFRP 3D printed parts. Despite of those, several advantages in part application for ball-milling were observed. Once one of gears failed, the corresponding jar doesn't spin anymore but others still works properly. This means one failed gear doesn't affect other gears because of its brittleness.

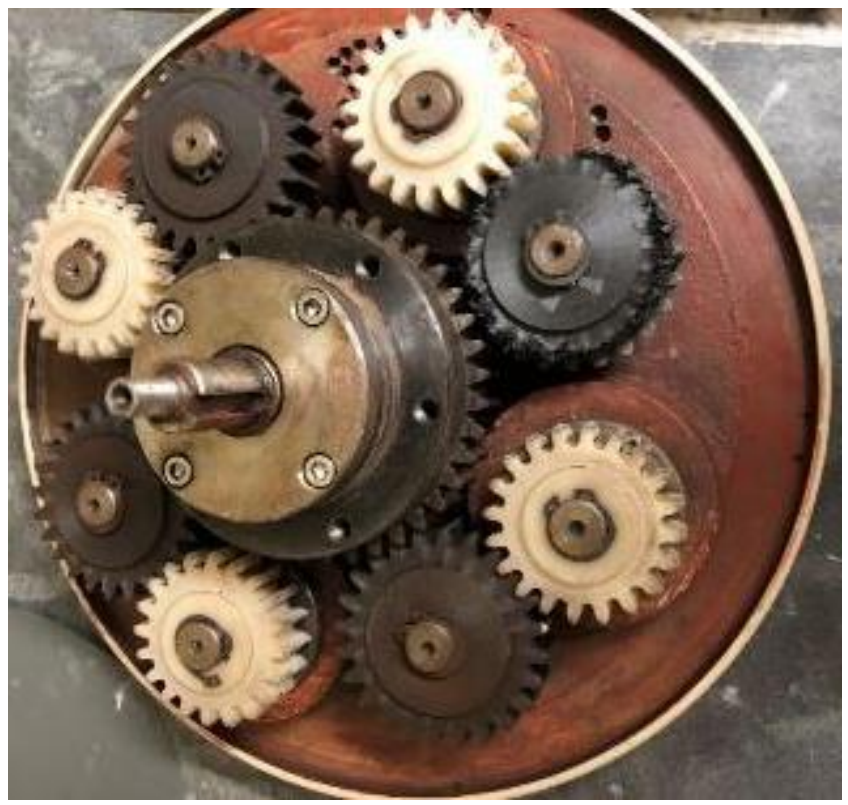

Figure 6-41 First trial of the application of CFRP gear set

For pure nylon gears however, they show high ductility, once one of gears failed, it deformed. Then the deformed gear cause unbalanced operation of ball-milling but still the operation sounds normal. Finally, all other gears are damaged once initial failure starts as shown in Figure 2.4. Therefore, in economic point of view, CFRP gears are better options considering that only one damaged gear is replaced. 


\subsubsection{Post-Processing - Spur Gear}

Annealing was performed for CFRP spur gear as a post-processing after 3D printing to improve structural properties. The method for thermoplastic 3D printed part recently has been developed by Stratasys [103]. However, the method for CFRP has not been developed. In this research, since the resin material of CFRP is Nylon 12, several annealing procedures for Nylon 12 were followed. The glass transition temperature of Nylon 12 is $141^{\circ} \mathrm{C}$ and melting temperature is $178 \sim 181^{\circ} \mathrm{C}$. Therefore, recommended annealing temperature has been determined as $140 \sim 160^{\circ} \mathrm{C}$ to have the material remove internal voids and release residual stress caused by manufacturing. The gear was placed on an oven safe surface for two hours to allow polymers chain realignment and recrystallization.

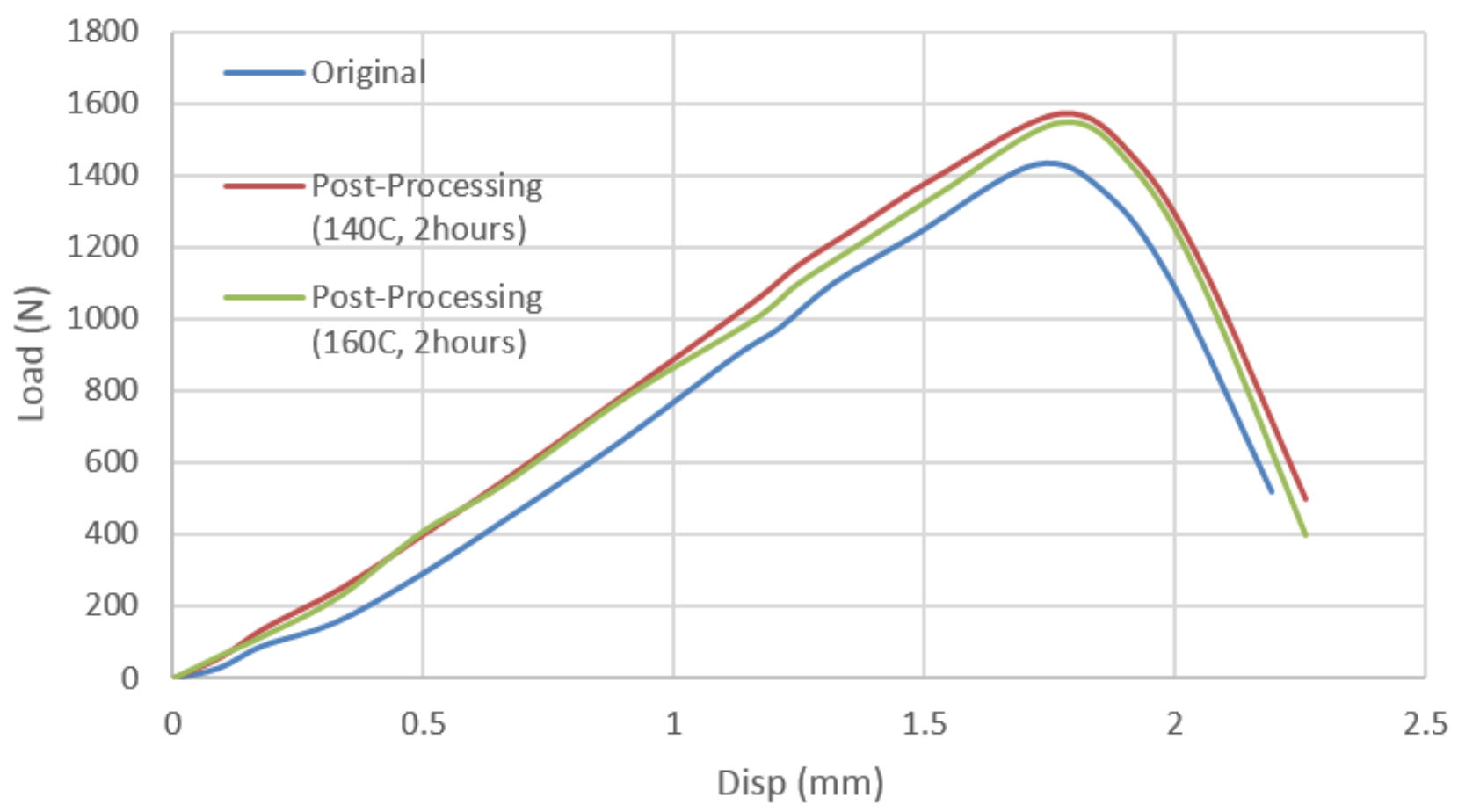

Figure 6-42 Stiffness test results of spur gears after post-processing

Relevant compression test for spur gear was performed again to see structural improvement by annealing. Figure 6-42 shows stiffness test results of gears after annealing with two different processing conditions. Failure strength, stiffness, and elongation were improved when compared with the original as $9 \%, 10 \%$, and $8 \%$, respectively. However, $20^{\circ} \mathrm{C}$ difference in annealing temperature doesn't play a role in the improvement. 


\section{Chapter 7 CONCLUSIONS AND FUTURE WORKS}

In this research, a novel methodology integrating SO, fiber placement theory, and tool-path development theory to design and manufacture AM parts has been successfully established through FEA and experimental validations. It starts with SO to reduce volume and determine the best geometry under the prescribed loading and boundary conditions. Then, stress field and principal directions were computed and optimal orientations of fibers were determined for individual elements. Using the output data from Abaqus $^{\mathrm{TM}}$, a tool-path optimization algorithm to maximize the effect of fiber reinforcement of CFRP was developed for FFF parts. Finally, finite element modeling was developed in order to apply orthotropic material properties which were determined from related lab coupon tests.

The proposed methodology demonstrates that the optimized tool-path extrudes fibers aligned with principal directions with high accuracy. Flow distribution of printed fibers was verified by SEM, which showed that approximately $83 \%$ of fibers were oriented as intended. Both numerical and experimental case study results show that CFRP parts printed by the optimized tool-path achieved approximately $8 \%$ improvement in structural performance over parts printed at uniform printing direction and showed $180 \%$ improvement over parts made of Nylon 12, respectively. However, physical experiments show $10 \%$ lower stiffness responses than those from FE simulations. To figure out this difference, voids and defects were characterized through microstructure analysis. In summary, assisted by SO and FEA, a customized 3D printing tool-path for CFRP has been developed with three different case studies to verify the proposed AM design methodology.

The proposed methodology can be extended for other materials especially for continuous fiber reinforced thermoplastic. Also, with the development of dual extruder system, dual material optimization would be an interesting topic in SO step. From the test result of actual application of CFRP engineering parts to a ball-milling machine, advantage and disadvantage of 3D printed CFRP parts caused by its brittleness were observed. Therefore, controlling the brittleness of CFRP in FFF manufacturing step can be another research area. Moreover, to predict its durability more accurately, fatigue tests are required. Due to the limitation of current FFF printing system, only in-plane tool-path optimization has been allowed in this research. However, if AM with tilted bed is fully developed, it may provide research opportunities for 3D tool-path optimization. 


\section{REFERENCES}

[1] M. P. Bendsøe and O. (Ole) Sigmund, Topology optimization : theory, methods, and applications. Springer, 2004.

[2] M. P. Bends e and N. Kikuchi, "Generating Optimal Topologies in Structural Design Using a Homogenization Method," Comput. Methods Appl. Mech. Eng., vol. 71, pp. 197-224, 1988.

[3] G. I. N. Rozvany, M. Zhou, and T. Birker, "Generalized shape optimization without homogenization,” Struct. Optim., vol. 4, no. 3-4, pp. 250-252, 1992.

[4] G. I. N. Rozvany, "A critical review of established methods of structural topology optimization," Struct. Multidiscip. Optim., vol. 37, no. 3, pp. 217-237, 2009.

[5] M. Zhou and G. I. N. Rozvany, "On the validity of ESO type methods in topology optimization," Structural and Multidisciplinary Optimization, vol. 21, no. 1. pp. 80-83, 2001.

[6] O. M. Querin, G. P. Steven, and Y. M. Xie, "Evolutionary structural optimisation using an additive algorithm," Finite Elem. Anal. Des., vol. 34, no. 3-4, pp. 291-308, 2000.

[7] Y. M. Xie and G. P. Steven, "A simple evolutionary procedure for structural optimization," Comput. Struct., vol. 49, no. 5, pp. 885-896, 1993.

[8] C. D. Chapman, K. Saitou, and M. J. Jakiela, "Genetic Algorithms as an Approach to Configuration and Topology Design,” J. Mech. Des., vol. 116, no. 4, p. 1005, 1994.

[9] C. D. Chapman and M. J. Jakiela, "Genetic algorithm-based structural topology design with compliance and topology simplification considerations," J. Mech. Des., vol. 118, no. 1, pp. 8998, 1996.

[10] A. Kaveh, B. Hassani, S. Shojaee, and S. M. Tavakkoli, "Structural topology optimization using ant colony methodology," Eng. Struct., vol. 30, no. 9, pp. 2559-2565, 2008.

[11] L. Xia, Q. Xia, X. Huang, and Y. M. Xie, "Bi-directional Evolutionary Structural Optimization on Advanced Structures and Materials: A Comprehensive Review," Arch. Comput. Methods Eng., pp. 1-42, 2016.

[12] M. P. Bendsøe, “Optimal shape design as a material distribution problem,” Struct. Optim., vol. 1, no. 4, pp. 193-202, 1989.

[13] O. M. Querin, G. P. Steven, and Y. M. Xie, "Evolutionary structural optimisation (ESO) using a bidirectional algorithm," Eng. Comput., vol. 15, no. 8, pp. 1031-1048, Dec. 1998.

[14] Z. B. Zabinsky, M. E. Tuttle, and C. Khompatraporn, "Chapter 21 A CASE STUDY : COMPOSITE S T R U C T U R E DESIGN OPTIMIZATION.”

[15] O. M. Querin, V. Young, G. P. Steven, and Y. M. Xie, "Computational efficiency and validation of bi-directional evolutionary structural optimization," Comput. Methods Appl. Mech. Eng., vol. 189, no. 2, pp. 559-573, 2000.

[16] X. Huang and Y. M. Xie, "Convergent and mesh-independent solutions for the bi-directional evolutionary structural optimization method," Finite Elem. Anal. Des., vol. 43, no. 14, pp. 10391049, 2007. 
[17] X. Huang and Y. M. Xie, "Evolutionary topology optimization of continuum structures with an additional displacement constraint," Struct. Multidiscip. Optim., vol. 40, no. 1-6, pp. 409-416, 2010.

[18] J. T. H. Sobieszczanski-Sobieski, "Multidisciplinary Aerospace Design Optimization : Survey of Recent Developments," Aiaa, vol. 53, no. 9, pp. 1689-1699, 2013.

[19] N. L. Pedersen, "Maximization of eigenvalues using topology optimization," Struct. Multidiscip. Optim., vol. 20, no. 1, pp. 2-11, 2000.

[20] J. Du and N. Olhoff, "Topological design of freely vibrating continuum structures for maximum values of simple and multiple eigenfrequencies and frequency gaps," Struct. Multidiscip. Optim., vol. 34, no. 2, pp. 91-110, 2007.

[21] X. Yang, Y. Xie, G. Steven, and O. Querin, “Topology Optimization for Frequencies Using an Evolutionary Method,” J. Struct. Eng., vol. 125, no. 12, pp. 1432-1438, 1999.

[22] A. Francavilla, C. V Ramakrishnan, and O. C. Zienkiewicz, "Optimization of shape to minimize stress concentration,” J. Strain Anal. Eng. Des., vol. 10, no. 2, pp. 63-70, 1975.

[23] V. Braibant and C. Fleury, "Shape optimal design using B-splines," Comput. Methods Appl. Mech. Eng., vol. 44, no. 3, pp. 247-267, 1984.

[24] R. T. Haftka and R. V. Grandhi, "Structural shape optimization-A survey," Comput. Methods Appl. Mech. Eng., vol. 57, no. 1, pp. 91-106, 1986.

[25] Y. Ding, "Shape optimization of structures: a literature survey," Comput. Struct., vol. 24, no. 6, pp. 985-1004, 1986.

[26] Y.-L. Hsu, “A review of structural shape optimization,” Comput. Ind., vol. 25, no. 1, pp. 3-13, 1994.

[27] S. S. Bhavikatti and C. V. Ramakrishnan, "Optimum shape design of rotating disks," Comput. Struct., vol. 11, no. 5, pp. 397-401, 1980.

[28] P. Pedersen and C. L. Laureen, "Design for Minimum Stress Concentration by Finite Elements and Linear Programming,” J. Struct. Mech., vol. 10, no. 4, pp. 375-391, 1982.

[29] C. Y. Kiyono, E. C. N. Silva, and J. N. Reddy, "A novel fiber optimization method based on normal distribution function with continuously varying fiber path," Compos. Struct., vol. 160, pp. 503-515, 2017.

[30] E. Lund, "Buckling topology optimization of laminated multi-material composite shell structures," Compos. Struct., vol. 91, no. 2, pp. 158-167, 2009.

[31] M. Bruyneel, "SFP-a new parameterization based on shape functions for optimal material selection: Application to conventional composite plies," Struct. Multidiscip. Optim., vol. 43, no. 1, pp. 17-27, 2011.

[32] T. Gao, W. Zhang, and P. Duysinx, "A bi-value coding parameterization scheme for the discrete optimal orientation design of the composite laminate," Int. J. Numer. Methods Eng., vol. 91, no. 1, pp. 98-114, 2012.

[33] J. P. Kruth, M. C. Leu, and T. Nakagawa, "Progress in additive manufacturing and rapid prototyping,” CIRP Ann. - Manuf. Technol., vol. 47, no. 2, pp. 525-540, 1998. 
[34] M. Burns, Automated fabrication : improving productivity in manufacturing. PTR Prentice Hall, 1993.

[35] C. K. Chua and K. F. Leong, 3D printing and additive manufacturing : principles and applications. .

[36] D. . Pham and R. . Gault, “A comparison of rapid prototyping technologies,” Int. J. Mach. Tools Manuf., vol. 38, no. 10-11, pp. 1257-1287, 1998.

[37] E. Sells, Z. Smith, S. Bailard, A. Bowyer, and V. Olliver, "RepRap: The Replicating Rapid Prototyper: Maximizing Customizability by Breeding the Means of Production," SSRN eLibrary, 2010.

[38] R. Jones et al., "RepRap - the replicating rapid prototyper," Robotica, vol. 29, no. 1, pp. 177191, 2011.

[39] A. Bowyer, "3D Printing and Humanity's First Imperfect Replicator," 3D Print. Addit. Manuf., vol. 1, no. 1, pp. 4-5, 2014.

[40] G. Rundle, A revolution in the making. .

[41] J. Moilanen and T. Vadén, "3D printing community and emerging practices of peer production," First Monday, vol. 18, no. 8, 2013.

[42] J. M. Pearce, C. Morris Blair, K. J. Laciak, R. Andrews, A. Nosrat, and I. Zelenika-Zovko, "3-D Printing of Open Source Appropriate Technologies for Self-Directed Sustainable Development," J. Sustain. Dev., vol. 3, no. 4, 2010.

[43] C. Mota, “The rise of personal fabrication," Proc. 8th ACM Conf. Creat. Cogn. - C\&C'11, p. 279, 2011.

[44] B. T. Wittbrodt et al., "Life-cycle economic analysis of distributed manufacturing with opensource 3-D printers," Mechatronics, vol. 23, no. 6, pp. 713-726, 2013.

[45] M. Kreiger and J. M. Pearce, "Environmental Impacts of Distributed Manufacturing from 3-D Printing of Polymer Components and Products," MRS Proc., vol. 1492, p. mrsf12-1492-g01-02, 2013.

[46] J. G. Tanenbaum, A. M. Williams, A. Desjardins, and K. Tanenbaum, "Democratizing technology: pleasure, utility and expressiveness in DIY and maker practice," Proc. SIGCHI Conf. Hum. Factors Comput. Syst., pp. 2603-2612, 2013.

[47] I. Mohomed and P. Dutta, "THE Age of DIY and Dawn of the Maker Movement," ACM SIGMOBILE Mob. Comput. Commun. Rev., vol. 18, no. 4, pp. 41-43, 2015.

[48] J. L. Irwin, J. M. Pearce, G. Anzalone, and D. E. Oppliger, “The RepRap 3-D Printer Revolution in STEM Education," in ASEE Annual Conference \& Exposition, 2014, p. 24.1242.1-24.1242.13.

[49] R. Thiago, L. Ferreira, I. Cardoso, T. Assis, and D. Bürger, "Experimental characterization and micrography of 3D printed PLA and PLA reinforced with short carbon fi bers," Compos. Part B, vol. 124, pp. 88-100, 2017.

[50] A. R. Torrado Perez, D. A. Roberson, and R. B. Wicker, "Fracture surface analysis of 3D-printed tensile specimens of novel ABS-based materials," Journal of Failure Analysis and Prevention, vol. 14, no. 3. pp. 343-353, 2014. 
[51] S. Hwang, E. I. Reyes, K. sik Moon, R. C. Rumpf, and N. S. Kim, “Thermo-mechanical Characterization of Metal/Polymer Composite Filaments and Printing Parameter Study for Fused Deposition Modeling in the 3D Printing Process," J. Electron. Mater., vol. 44, no. 3, pp. 771777, 2015.

[52] W. Zhong, F. Li, Z. Zhang, L. Song, and Z. Li, "Short fiber reinforced composites for fused deposition modeling," Mater. Sci. Eng. A301, vol. 301, pp. 125-130, 2001.

[53] R. W. Gray, D. G. Baird, and J. H. Bohn, "Thermoplastic composites reinforced with long fiber thermotropic liquid crystalline polymers for fused deposition modeling," Polym. Compos., vol. 19, no. 4, pp. 383-394, 1998.

[54] M. L. Shofner, K. Lozano, F. J. Rodríguez-Macías, and E. V. Barrera, "Nanofiber-reinforced polymers prepared by fused deposition modeling," J. Appl. Polym. Sci., vol. 89, no. 11, pp. 3081-3090, 2003.

[55] S. Dul, L. Fambri, and A. Pegoretti, "Fused deposition modelling with ABS-graphene nanocomposites," Compos. Part A Appl. Sci. Manuf., vol. 85, pp. 181-191, 2016.

[56] R. Matsuzaki et al., "Three-dimensional printing of continuous-fiber composites by in-nozzle impregnation," Sci. Rep., vol. 6, no. 1, p. 23058, 2016.

[57] F. Van Der Klift, Y. Koga, A. Todoroki, M. Ueda, Y. Hirano, and R. Matsuzaki, "3D Printing of Continuous Carbon Fibre Reinforced Thermo-Plastic (CFRTP) Tensile Test Specimens," Open J. Compos. Mater., vol. 6, no. 1, pp. 18-27, 2016.

[58] S. Fakirov and C. Fakirova, "Direct determination of the orientation of short glass fibers in an injection-molded poly(ethylene terephthalate) system," Polym. Compos., vol. 6, no. 1, pp. 41-46, 1985.

[59] M. Vincent and J. F. Agassant, "Experimental study and calculations of short glass fiber orientation in a center gated molded disc," Polym. Compos., vol. 7, no. 2, pp. 76-83, 1986.

[60] G. Fischer and P. Eyerer, "Measuring spatial orientation of short fiber reinforced thermoplastics by image analysis," Polym. Compos., vol. 9, pp. 297-304, 1988.

[61] R. S. Bay and C. L. Tucker, "Stereological measurement and error estimates for threedimensional fiber orientation," Polym. Eng. Sci., vol. 32, no. 4, pp. 240-253, 1992.

[62] G. Zak, C. B. Park, and B. Benhabib, "Estimation of three-dimensional fibre-orientation distribution in short-fibre composites by a two-section method," J. Compos. Mater., vol. 35, no. 4, pp. 316-339, 2001.

[63] C. Eberhardt and A. Clarke, "Fibre-orientation measurements in short-glass-fibre composites. Part I: Automated, high-angular-resolution measurement by confocal microscopy," Compos. Sci. Technol., vol. 61, no. 10, pp. 1389-1400, 2001.

[64] S. H. McGee and R. L. McCullough, "Characterization of fiber orientation in short-fiber composites,” J. Appl. Phys., vol. 55, no. 5, pp. 1394-1403, 1984.

[65] J. W. Kim and D. G. Lee, "Measurement of fiber orientation angle in FRP by intensity method," J. Mater. Process. Technol., vol. 201, no. 1-3, pp. 755-760, 2008.

[66] H. Shen, S. Nutt, and D. Hull, "Direct observation and measurement of fiber architecture in short fiber-polymer composite foam through micro-CT imaging," Compos. Sci. Technol., vol. 64, no. 
13-14, pp. 2113-2120, 2004.

[67] L. P. Djukic, G. M. Pearce, I. Herszberg, M. K. Bannister, and D. H. Mollenhauer, "Contrast enhancement of microct scans to aid 3D modelling of carbon fibre fabric composites," Appl. Compos. Mater., vol. 20, no. 6, pp. 1215-1230, 2013.

[68] L. P. Djukic, I. Herszberg, W. R. Walsh, G. A. Schoeppner, and B. Gangadhara Prusty, "Contrast enhancement in visualisation of woven composite architecture using a MicroCT Scanner. Part 2: Tow and preform coatings," Compos. Part A Appl. Sci. Manuf., vol. 40, no. 12, pp. 1870-1879, 2009.

[69] A. E. Scott, I. Sinclair, S. M. Spearing, M. N. Mavrogordato, and W. Hepples, "Influence of voids on damage mechanisms in carbon/epoxy composites determined via high resolution computed tomography," Compos. Sci. Technol., vol. 90, pp. 147-153, 2014.

[70] H. L. Tekinalp et al., "Highly oriented carbon fiber - polymer composites via additive manufacturing," Compos. Sci. Technol., vol. 105, pp. 144-150, 2014.

[71] S. Ahn, M. Montero, D. Odell, S. Roundy, and P. K. Wright, "Anisotropic material properties of fused deposition modeling ABS," Rapid Prototyp. J., vol. 8, no. 4, pp. 248-257, 2002.

[72] A. Bellini and S. Guceri, "Mechanical characterization of parts fabricated using fused deposition modeling," Rapid Prototyp. J., vol. 9, no. 4, pp. 252-264, 2003.

[73] L. J. Love et al., "The importance of carbon fiber to polymer additive manufacturing," J. Mater. Res., vol. 29, no. 17, pp. 1893-1898, 2014.

[74] F. Ning, W. Cong, J. Qiu, J. Wei, and S. Wang, "Additive manufacturing of carbon fiber reinforced thermoplastic composites using fused deposition modeling," Compos. Part B Eng., vol. 80, pp. 369-378, 2015.

[75] F. Ning, W. Cong, Y. Hu, and H. Wang, "Additive manufacturing of carbon fiber-reinforced plastic composites using fused deposition modeling: Effects of process parameters on tensile properties," J. Compos. Mater., vol. 51, no. 4, pp. 451-462, 2017.

[76] G. W. Melenka, B. K. O. O. Cheung, J. S. Schofield, M. R. Dawson, and J. P. Carey, "Evaluation and prediction of the tensile properties of continuous fiber-reinforced 3D printed structures," Compos. Struct., vol. 153, pp. 866-875, 2016.

[77] M. A. S. Anwer and H. E. Naguib, "Study on the morphological, dynamic mechanical and thermal properties of PLA carbon nanofibre composites," Compos. Part B Eng., vol. 91, pp. 631639, 2016.

[78] A. Jaszkiewicz, A. Meljon, A. K. Bledzki, and M. Radwanski, "Gaining knowledge on the processability of PLA-based short-fibre compounds - A comprehensive comparison with their PP counterparts," Compos. Part A Appl. Sci. Manuf., vol. 83, pp. 140-151, 2016.

[79] N. Li, Y. Li, and S. Liu, "Rapid prototyping of continuous carbon fiber reinforced polylactic acid composites by 3D printing," J. Mater. Process. Technol., vol. 238, pp. 218-225, 2016.

[80] X. Tian, T. Liu, C. Yang, Q. Wang, and D. Li, "Interface and performance of 3D printed continuous carbon fiber reinforced PLA composites," Compos. Part A Appl. Sci. Manuf., vol. 88, pp. 198-205, 2016.

[81] X. Tian, T. Liu, Q. Wang, A. Dilmurat, D. Li, and G. Ziegmann, "Recycling and 
remanufacturing of 3D printed continuous carbon fiber reinforced PLA composites," J. Clean. Prod., vol. 142, pp. 1609-1618, 2017.

[82] X. Yao, C. Luan, D. Zhang, L. Lan, and J. Fu, "Evaluation of carbon fiber-embedded 3D printed structures for strengthening and structural-health monitoring," Mater. Des., vol. 114, pp. 424432, 2017.

[83] M. De Almeida, M. Cerqueira, and M. Leali, "The influence of porosity on the interlaminar shear strength of carbon / epoxy and carbon / bismaleimide fabric laminates," Compos. Sci. Technol., vol. 61, pp. 2101-2108, 2001.

[84] S. F. M. de Almeida and Z. dos S. N. Neto, "Effect of void content on the strength of composite laminates," Compos. Struct., vol. 28, no. 2, pp. 139-148, 1994.

[85] D. M. Daniel, S. C. Wooh, and I. Komsky, "Quantitative porosity characterization of composite materials by means of ultrasonic attenuation measurements," J. Nondestruct. Eval., vol. 11, no. 1, pp. 1-8, 1992.

[86] A. H. Kite, D. K. Hsu, and D. J. Barnard, "Determination of porosity content in composites by micrograph image processing," in AIP Conference Proceedings, 2008, vol. 975, pp. 942-949.

[87] H. Zhu, B. Wu, D. Li, D. Zhang, and Y. Chen, "Influence of Voids on the Tensile Performance of Carbon/epoxy Fabric Laminates," J. Mater. Sci. Technol., vol. 27, no. 1, pp. 69-73, 2011.

[88] J. Kastner, B. Plank, D. Salaberger, and J. Sekelja, "Defect and Porosity Determination of Fibre Reinforced Polymers by X-ray Computed Tomography," NDT Aerosp. 2010 - We.1.A.2, pp. 112, 2010.

[89] K. Tata, G. Fadel, A. Bagchi, and N. Aziz, "Efficient slicing for layered manufacturing," Rapid Prototyp. J., vol. 4, no. 4, pp. 151-167, Dec. 1998.

[90] J. Tyberg and J. H. Bøhn, “Local adaptive slicing,” Rapid Prototyp. J., vol. 4, no. 3, pp. 118-127, 1998.

[91] P. Mohan Pandey, N. Venkata Reddy, and S. G. Dhande, "Slicing procedures in layered manufacturing: a review," Rapid Prototyp. J., vol. 9, no. 5, pp. 274-288, 2003.

[92] W. Han, M. A. Jafari, S. C. Danforth, and A. Safari, "Tool Path-Based Deposition Planning in Fused Deposition Processes,” J. Manuf. Sci. Eng., vol. 124, no. 2, p. 462, 2002.

[93] J. Kao and F. B. Prinz, "Optimal Motion Planning for Deposition in Layered Manufacturing," 1998 ASME Des. Eng. Tech. Conf., p. 10, 1998.

[94] Y. Yang, H. T. Loh, J. Y. H. Fuh, and Y. G. Wang, "Equidistant path generation for improving scanning efficiency in layered manufacturing," Rapid Prototyp. J., vol. 8, no. 1, pp. 30-37, 2002.

[95] Y. W. Y. Weidong, "Optimal path planning in Rapid Prototyping based on genetic algorithm," 2009 Chinese Control Decis. Conf., pp. 5068-5072, 2009.

[96] P. K. WAH, K. G. MURTY, A. JONEJA, and L. C. CHIU, "Tool path optimization in layered manufacturing," IIE Trans., vol. 34, no. 4, pp. 335-347, 2002.

[97] G. Q. Jin, W. D. Li, and L. Gao, “An adaptive process planning approach of rapid prototyping and manufacturing," Robot. Comput. Integr. Manuf., vol. 29, no. 1, pp. 23-38, 2013.

[98] T. T. El-Midany, A. Elkeran, and H. Tawfik, “Toolpath pattern comparison contour-parallel with 
direction-parallel," in Geometric Modeling and Imaging New Trends, 2006, 2006, vol. 2006, pp. 77-82.

[99] B. Babatope and D. H. Isaac, "Annealing of isotropic nylon-6,6," Polymer (Guildf)., vol. 33, no. 8, pp. 1664-1668, 1992.

[100] "Mechanical properties of components fabricated with open-source 3-D printers under realistic environmental conditions," Mater. Des., vol. 58, pp. 242-246, Jun. 2014.

[101] P. Materials and E. I. Materials, "Standard Test Method for Tensile Properties of Plastics 1," pp. $1-17,2017$.

[102] D 3518, "Standard Test Method for In-Plane Shear Response of Polymer Matrix Composite Materials by Tensile Test of a $645^{\circ}$ Laminate 1," Annu. B. ASTM Stand., vol. 94, no. Reapproved, pp. 1-7, 2007.

[103] F. D. M. B. Practice, "Nylon 12," vol. 55, p. 104.

[104] X. Huang, Z. H. Zuo, and Y. M. Xie, "Evolutionary topological optimization of vibrating continuum structures for natural frequencies," Comput. Struct., vol. 88, no. 5-6, pp. 357-364, 2010 .

[105] Z. H. Zuo and Y. M. Xie, "Evolutionary topology optimization of continuum structures with a global displacement control," CAD Comput. Aided Des., vol. 56, pp. 58-67, 2014.

[106] J. Haslinger and R. A. E. Mäkinen, Introduction to Shape Optimization. Society for Industrial and Applied Mathematics, 2003.

[107] "2. A Mathematical Introduction to Sizing and Shape Optimization," in Introduction to Shape Optimization, Society for Industrial and Applied Mathematics, 2003, pp. 13-95.

[108] “3. Sensitivity Analysis," in Introduction to Shape Optimization, Society for Industrial and Applied Mathematics, 2003, pp. 99-127.

[109] J. Stegmann and E. Lund, "Discrete material optimization of general composite shell structures," no. February 2005, pp. 2009-2027, 2009.

[110] I. Gibson and D. Rosen, Additive Manufacturing Technologies. .

[111] P. Zhang, J. Liu, and A. C. To, "Scripta Materialia Role of anisotropic properties on topology optimization of additive manufactured load bearing structures," Scr. Mater., vol. 135, pp. 148$152,2017$.

[112] C. Koch, L. Van Hulle, and N. Rudolph, "Investigation of mechanical anisotropy of the fused filament fabrication process via customized tool path generation," Addit. Manuf., vol. 16, pp. 138-145, 2017.

[113] A. F. Abd El-Rehim and H. Y. Zahran, "Investigation of microstructure and mechanical properties of Sn-xCu solder alloys," J. Alloys Compd., vol. 695, pp. 3666-3673, 2017.

[114] Y. Jin, Y. He, G. Fu, A. Zhang, and J. Du, "A non-retraction path planning approach for extrusion-based additive manufacturing," Robot. Comput. Integr. Manuf., vol. 48, no. August 2016, pp. 132-144, 2017.

[115] Italo Soares, "Most used 3D Printer G-Codes - Commands detailed," 3D Printer G-CODES The Newbie Guide, 2017. [Online]. Available: https://3dprinterchat.com/2016/02/3d-printer-g- 
codes/. [Accessed: 28-Sep-2017].

[116] Ed Tyson, "How to Anneal Your 3d Prints for Strength," rigid.ink, 2017. [Online]. Available: https://rigid.ink/blogs/news/how-to-anneal-your-3d-prints-for-strength. [Accessed: 01-Jun-2018].

[117] Pinterest, "Drone Frames - CF DRQ250 \&quot;Mini-D\&quot; - Carbon Fiber | Mini-Drone FPV 4K Project | Pinterest | Dji phantom and Open source," Explore Carbon Fiber, Quad, and more, 2017. [Online]. Available: https://www.pinterest.com/pin/302867143667155667/. [Accessed: 28Nov-2017].

[118] Y. Jin, Y. He, J. Fu, W. Gan, and Z. Lin, "generation for material extrusion-based additive manufacturing technology §optimization of tool-path," Addit. Manuf., vol. 1-4, pp. 32-47, 2014. 


\section{APPENDIX}

A. Tensile test results -Nlyon 12

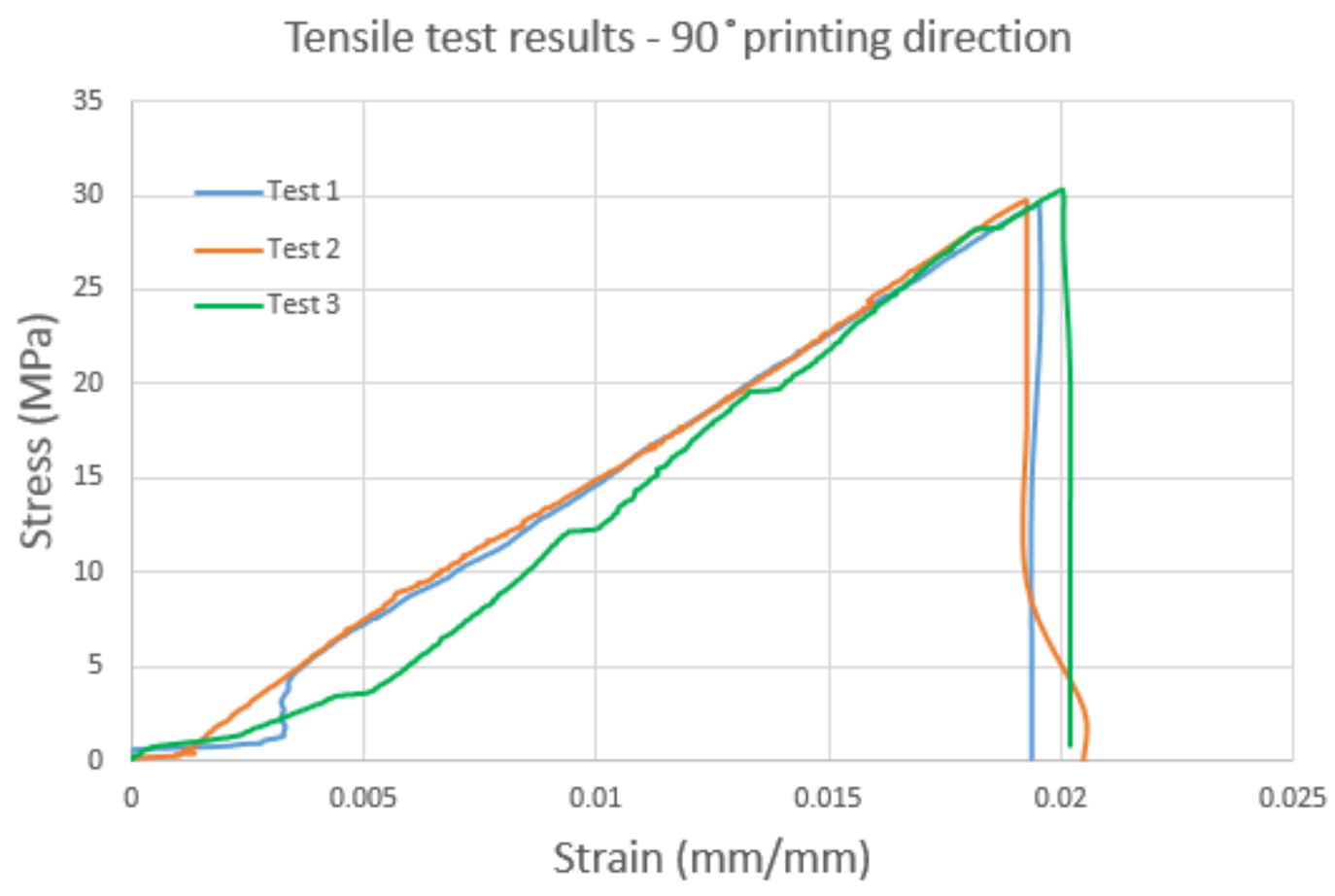

Figure 7-1 Strain vs stress - specimens printed 90

Tensile test results $-45^{\circ}$ printing direction

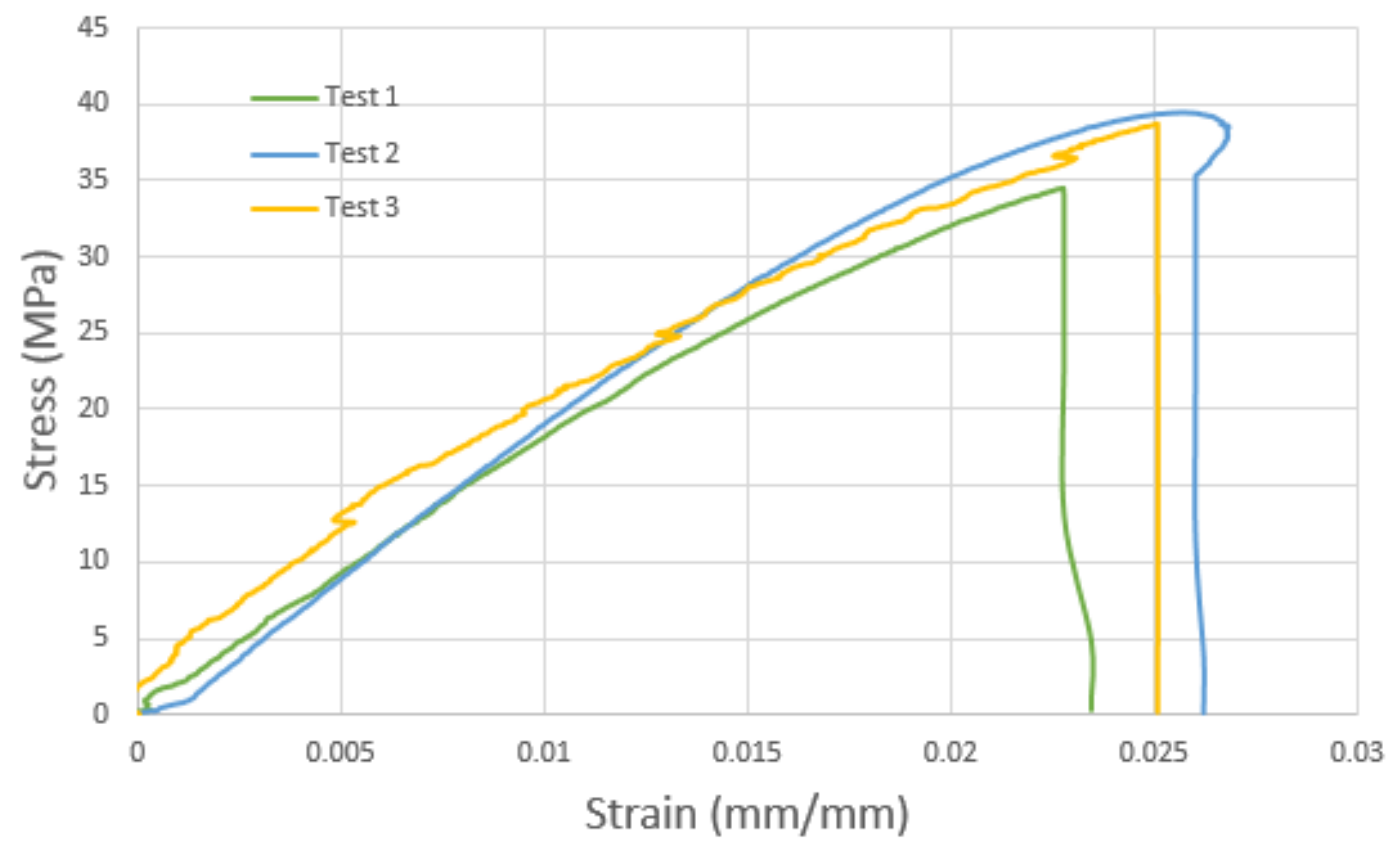

Figure 7-2 Strain vs stress - specimens printed $45^{\circ}$ 
Tensile test result $-0^{\circ}$ printing direction

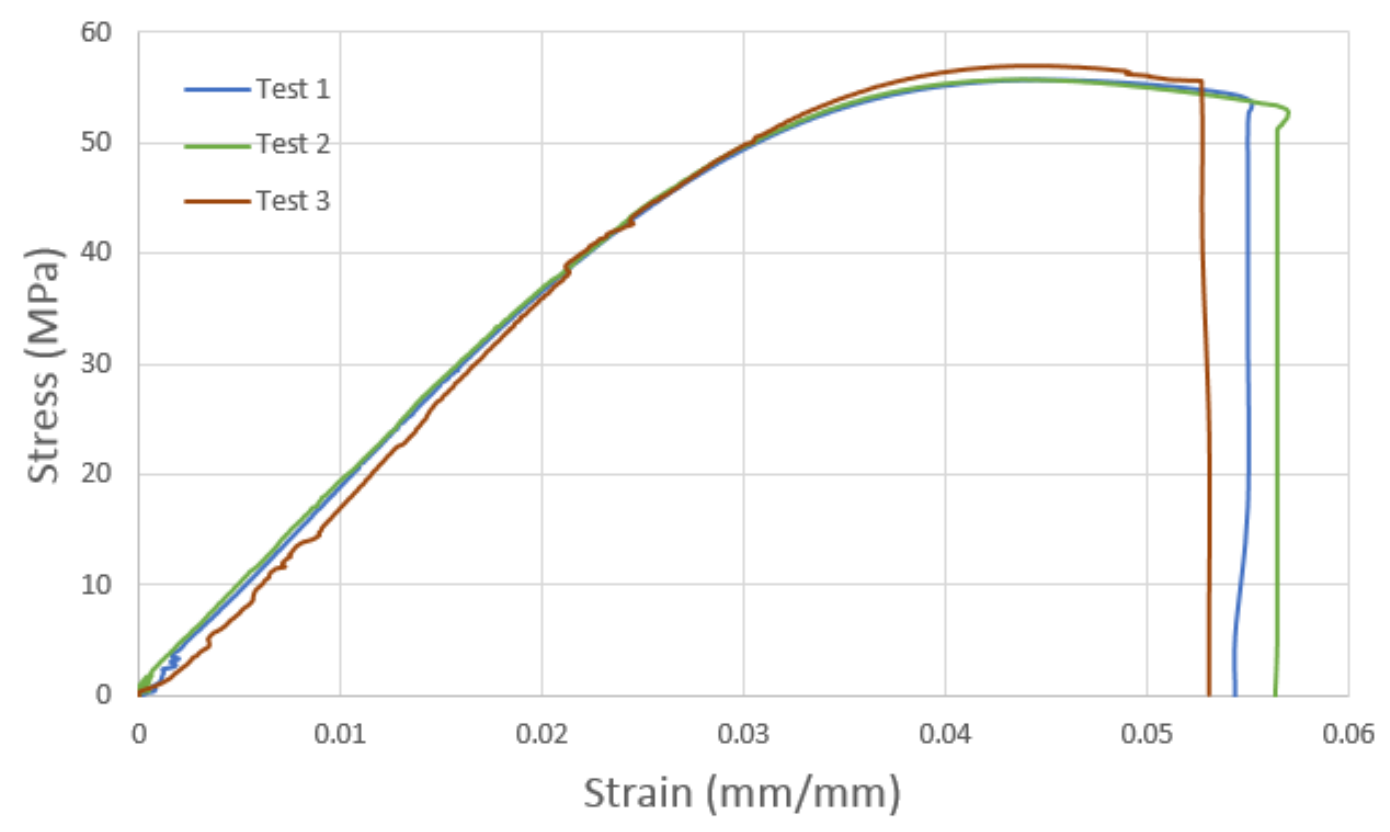

Figure 7-3 Strain vs stress - sepcimens printed $0^{\circ}$ 


\section{B. Matlab codes - Tool-path development}

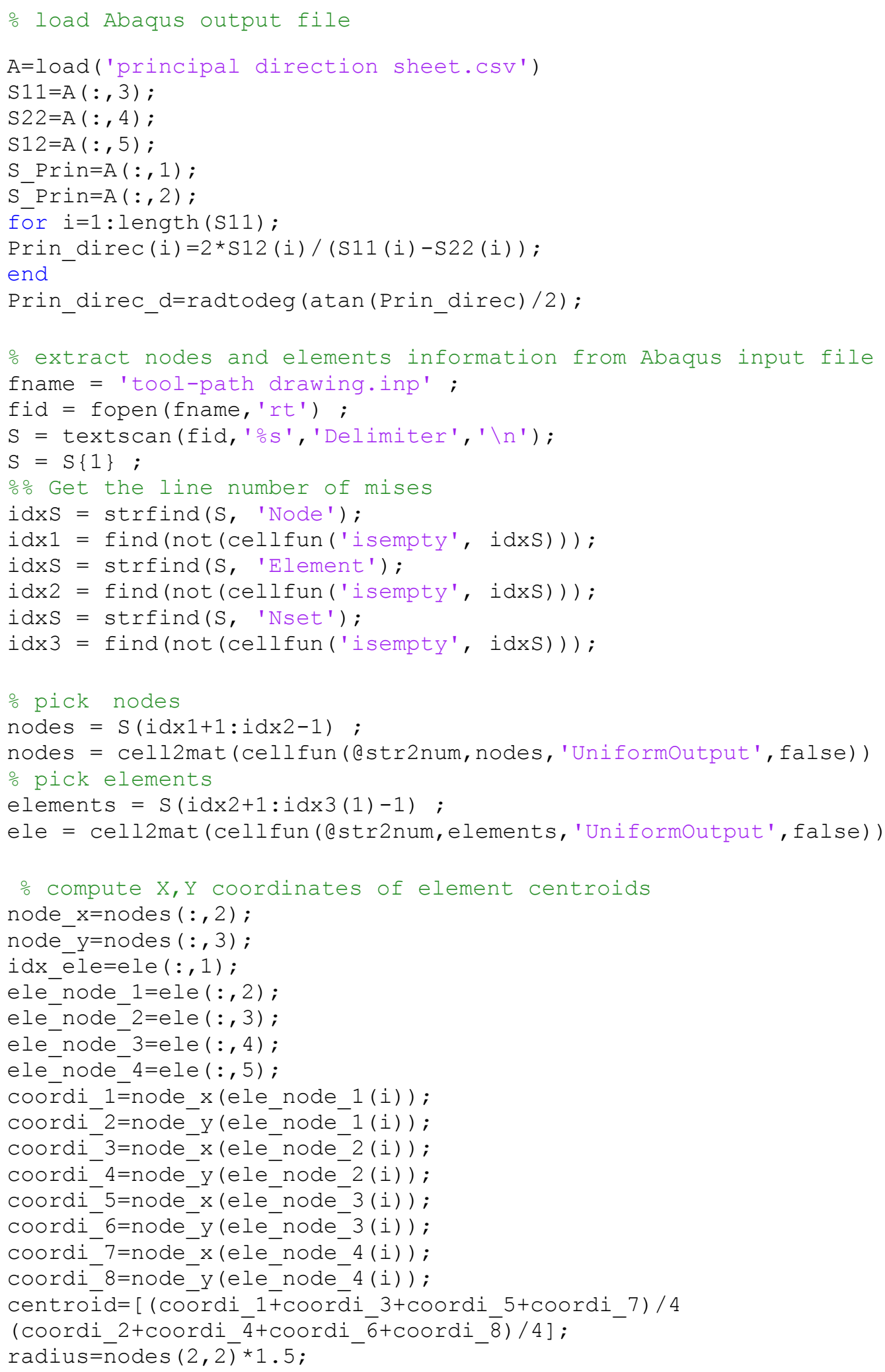




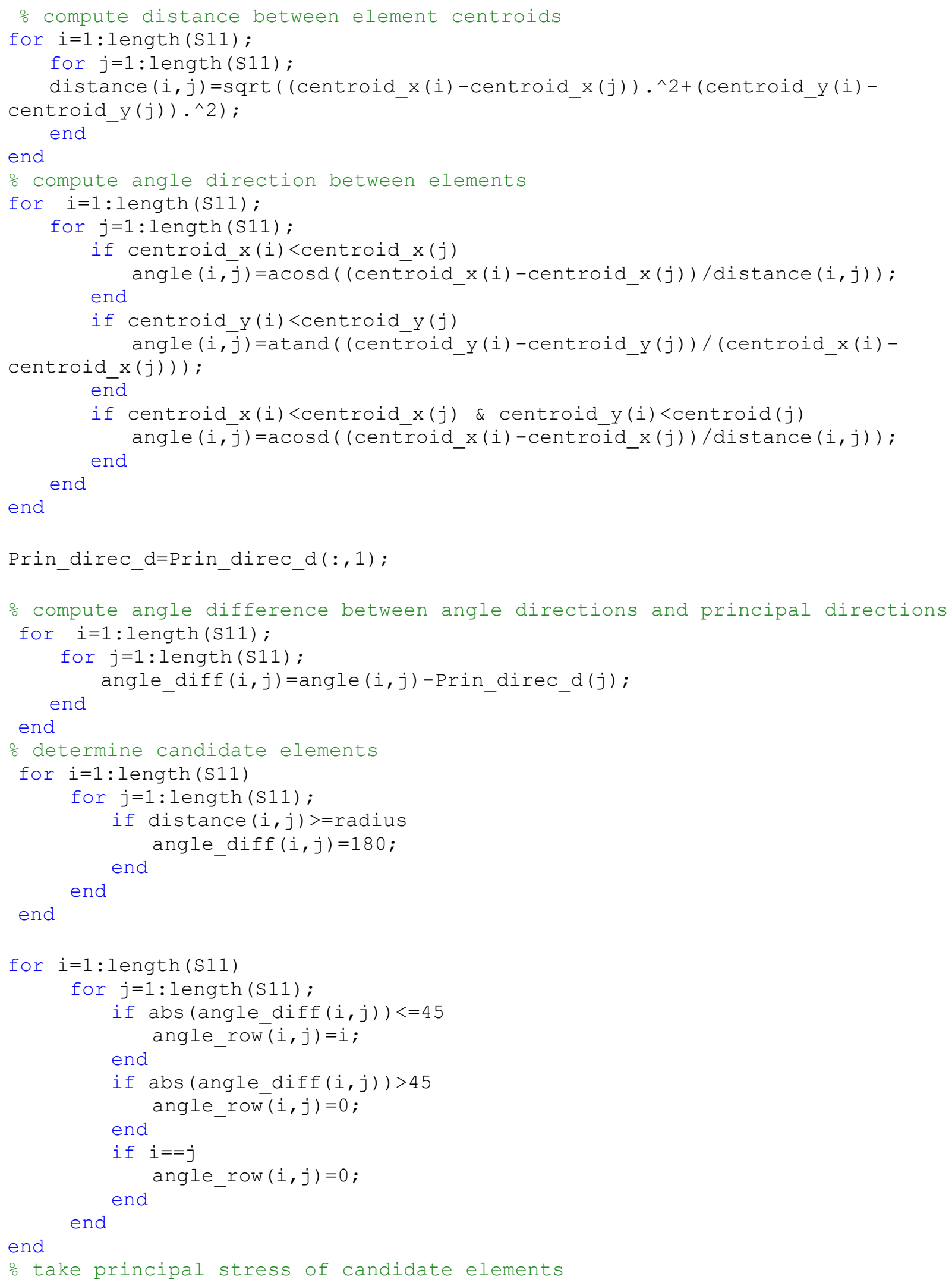




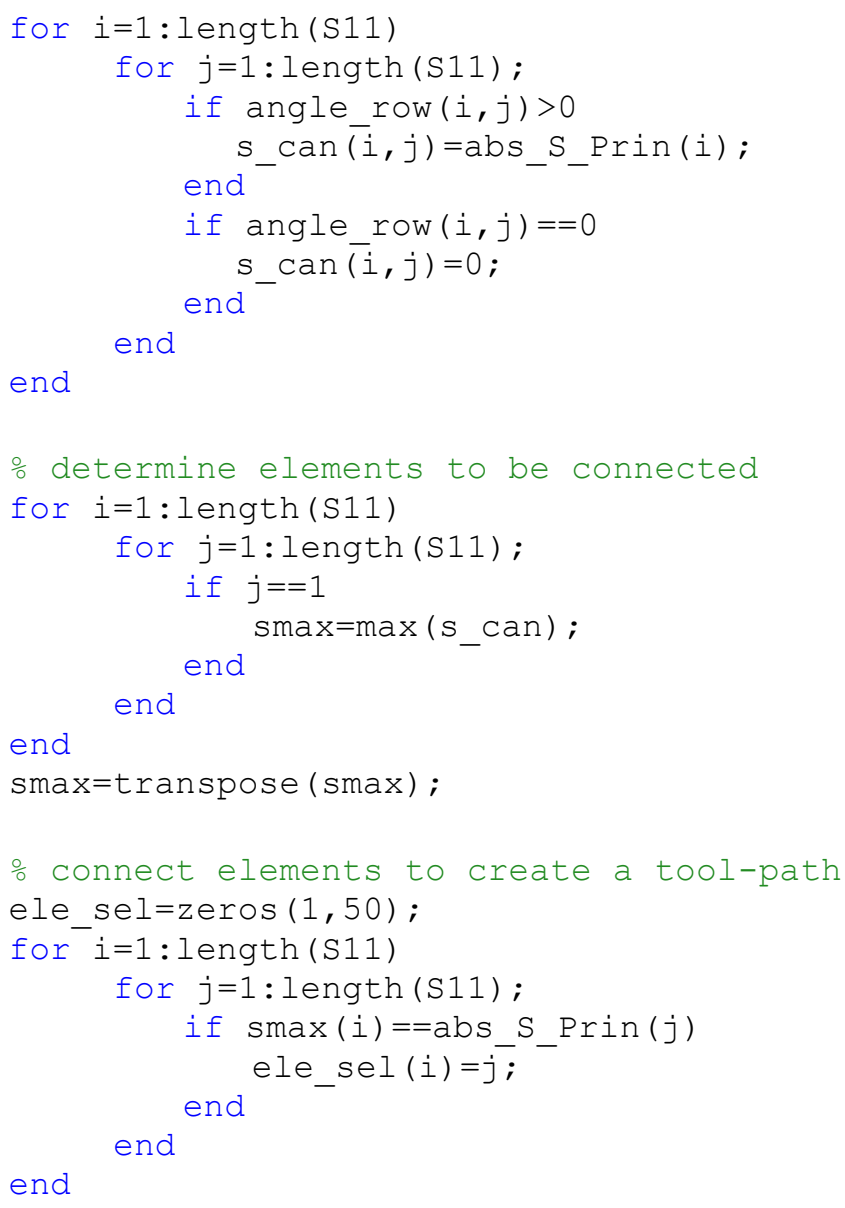


C. CFRP-nylon material properties

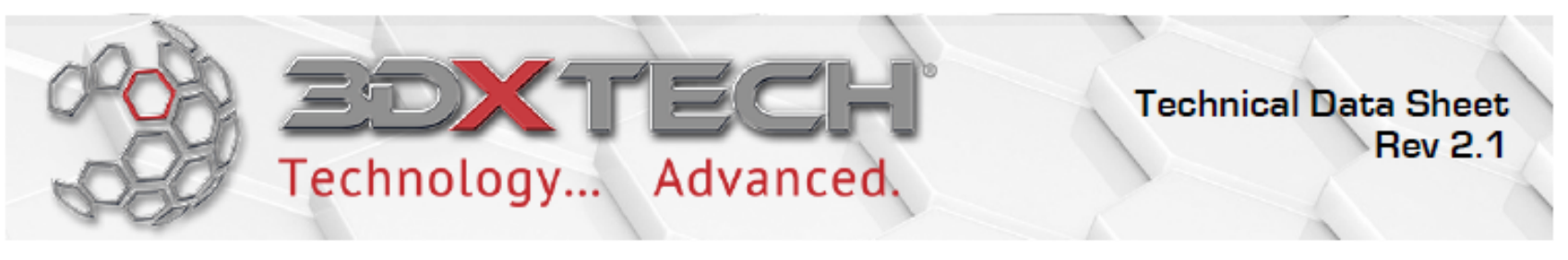

\section{CarbonX $X^{\mathrm{TM}}$ Carbon Fiber Reinforced Nylon 3D Filament}

CarbonX ${ }^{\mathrm{TM}}$ Carbon Fiber Reinforce Nylon is a high-performance carbon fiber reinforced 3D printing filament. This grade was formulated utilizing high-modulus carbon fiber and an advanced semi-aromatic polyamide copolymer - making it ideal for applications that require superior chemical resistance, thermal properties, mechanical properties, and dimensional stability compared to other FDM/FFF nylon materials. Suitable for use in practically all consumer-grade FDM/FFF printers that have a heated print bed. Made 3DXTECH $(\AA)$ in the USA.

The reported technical data was generated from printed ISO test specimen. The general print parameters utilized are noted below.

- Desktop FDM/FFF Printer

- Nozzle: 0.4mm A2 hardened

- Layer height: $0.2 \mathrm{~mm}$

- Infill: 100\%, +/-45

- Extrusion temp: $260^{\circ} \mathrm{C}$

- Bed temp: $110^{\circ} \mathrm{C}$

- Bed prep: ABS/Acetone Gel

- Print speed: 50 mm/sec

\begin{tabular}{lllc} 
General Property & Unit & Standard & Typical Value \\
\hline Density & $\mathrm{g} / \mathrm{cc}$ & ISO 1183 & 1.14 \\
Mechanical Property & Unit & Standard & Typical Value \\
\hline Tensile Strength & $\mathrm{MPa}$ & ISO 527 & 63.9 \\
\hline Tensile Modulus & $\mathrm{MPa}$ & ISO 527 & 4387 \\
\hline Tensile Elongation, Break & $\%$ & ISO 527 & 4 \\
\hline Flexural Modulus & $\mathrm{MPa}$ & ISO 178 & 5650 \\
\hline Flexural Strength & $\mathrm{MPa}$ & ISO 178 & 78 \\
\hline
\end{tabular}


D. Nylon 12 material properties

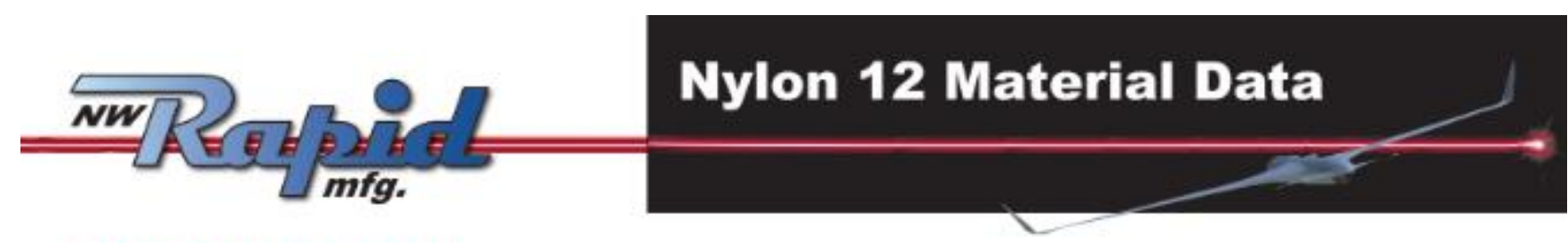

PRODUCT DESCRIPTION

Our Standard Nylon 12 material is ideal for most applications. It is lightweight, tough, flexible yet rigid, and economical. Nylon 12 is our choice for prototypes and production parts. Nylon 12 in compliance with FDA 21 CFR, $\$ 177.15009$ (b) except for alcoholic foodstuff. Nylon 12 has no porosity with wall thicknesses greater than $0.060^{\prime \prime}(1.5 \mathrm{~mm})$, has exceptional chemical and fuel resistance, and can be used for fuel and liquid storage. We have examples that have held 10psi for longer than two years with no fuel loss.

\begin{tabular}{|c|c|c|c|}
\hline MECHANICAL PROPERTIES & VALUE & UNIT & TEST STANDARD \\
\hline Flexural Modulus $\left(23^{\circ} \mathrm{C}\right)$ & 1500 & $\mathrm{MPa}$ & ISO 178 \\
\hline Flexural Strength & 58 & $\mathrm{MPa}$ & ISO 178 \\
\hline Izod Impact Notched $\left(23^{\circ} \mathrm{C}\right)$ & 4.4 & $\mathrm{~kJ} / \mathrm{m}^{2}$ & ISO 180/1A \\
\hline Izod Impact Unnotched $\left(23^{\circ} \mathrm{C}\right)$ & 33 & $\mathrm{~kJ} / \mathrm{m}^{2}$ & ISO $180 / 1 \mathrm{U}$ \\
\hline Shore D Hardness (15s) & 75 & - & ISO 868 \\
\hline Ball Indentation Hardness & 78 & $\mathrm{MPa}$ & ISO 2039-1 \\
\hline 3D DATA & VALUE & UNIT & TEST STANDARD \\
\hline \multicolumn{4}{|c|}{$\begin{array}{l}\text { The properties of parts manufactured using laser siniering are due bo their layer-by-layer production, to some extent direction } \\
\text { dependent. This has to be considered with designing the part and defining the build orientation. }\end{array}$} \\
\hline Tensile Modulus (X Direction) & 1700 & $\mathrm{MPa}$ & ISO $527-1 /-2$ \\
\hline Tensile Modulus (Y Direction) & 1700 & $\mathrm{MPa}$ & ISO $527-1 /-2$ \\
\hline Tensile Strength (X Direction) & 48 & $\mathrm{MPa}$ & ISO $527-1 /-2$ \\
\hline Tensile Strength (Y Direction) & 48 & $\mathrm{MPa}$ & ISO $527-1 /-2$ \\
\hline Strain at Break (X Direction) & 15 & $\%$ & ISO $527-1 /-2$ \\
\hline Charpy Impact Strength $\left(+23^{\circ} \mathrm{C}, \mathrm{X}\right.$ Direction $)\left(+23^{\circ} \mathrm{C}\right)$ & 53 & $\mathrm{k} J \mathrm{~J} / \mathrm{m}^{2}$ & ISO $179 / 1 \mathrm{eU}$ \\
\hline Charpy Notched impact Strength $\left(+23^{\circ} \mathrm{C}, \mathrm{X}\right.$ Direction) $\left(+23^{\circ} \mathrm{C}\right)$ & 4.8 & $\mathrm{kJJ} / \mathrm{m}^{2}$ & ISO $179 / 1$ ea \\
\hline
\end{tabular}

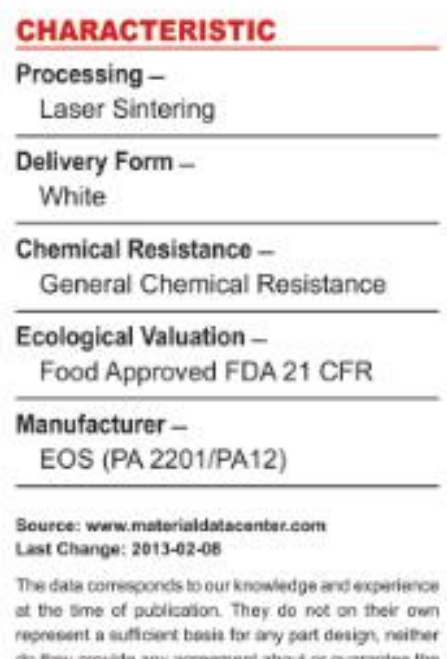


E. SEM images of Nylon 12

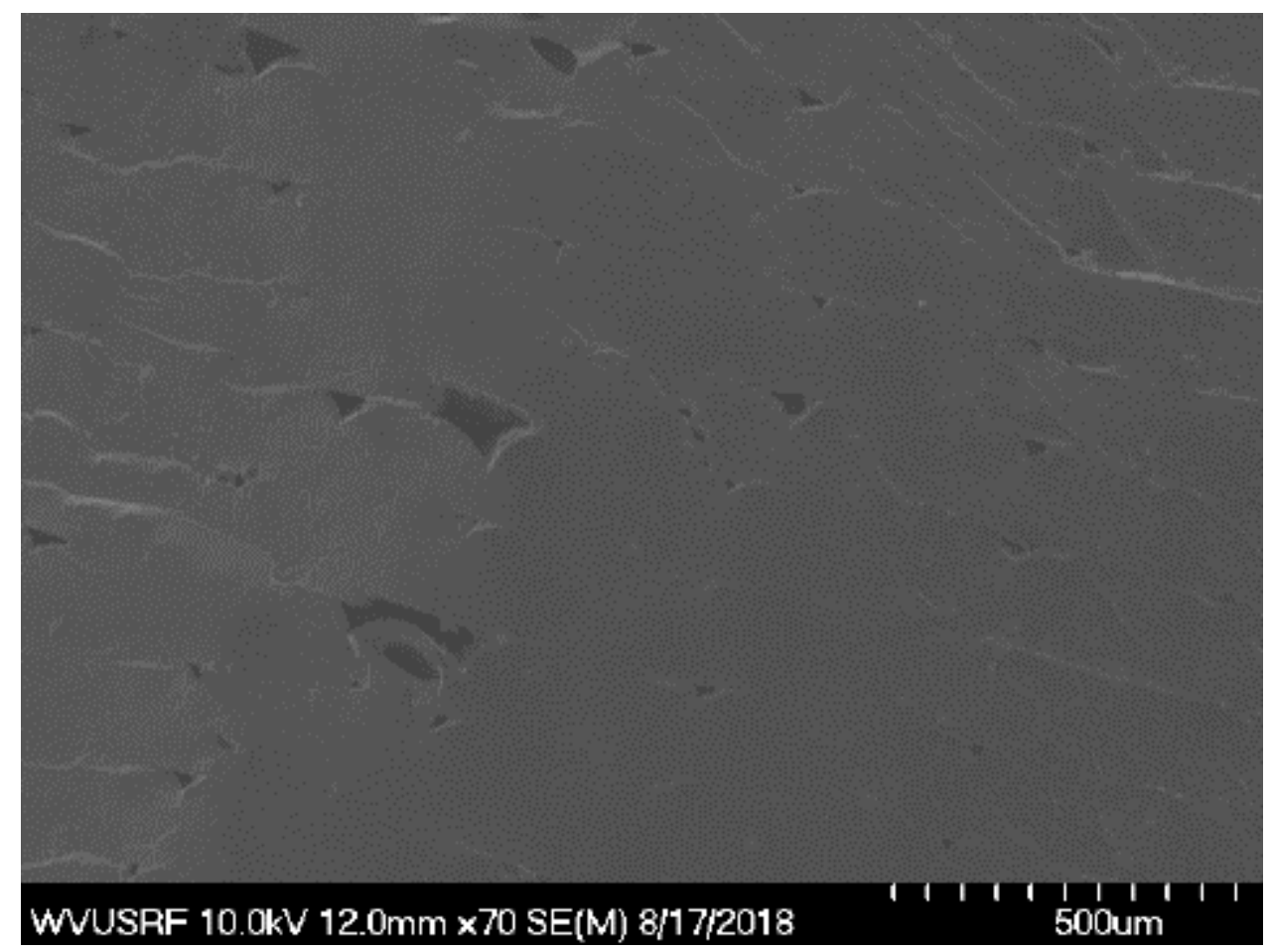

Figure 7-4 SEM image of a sample printed by $0.4 \mathrm{~mm}$ nozzle

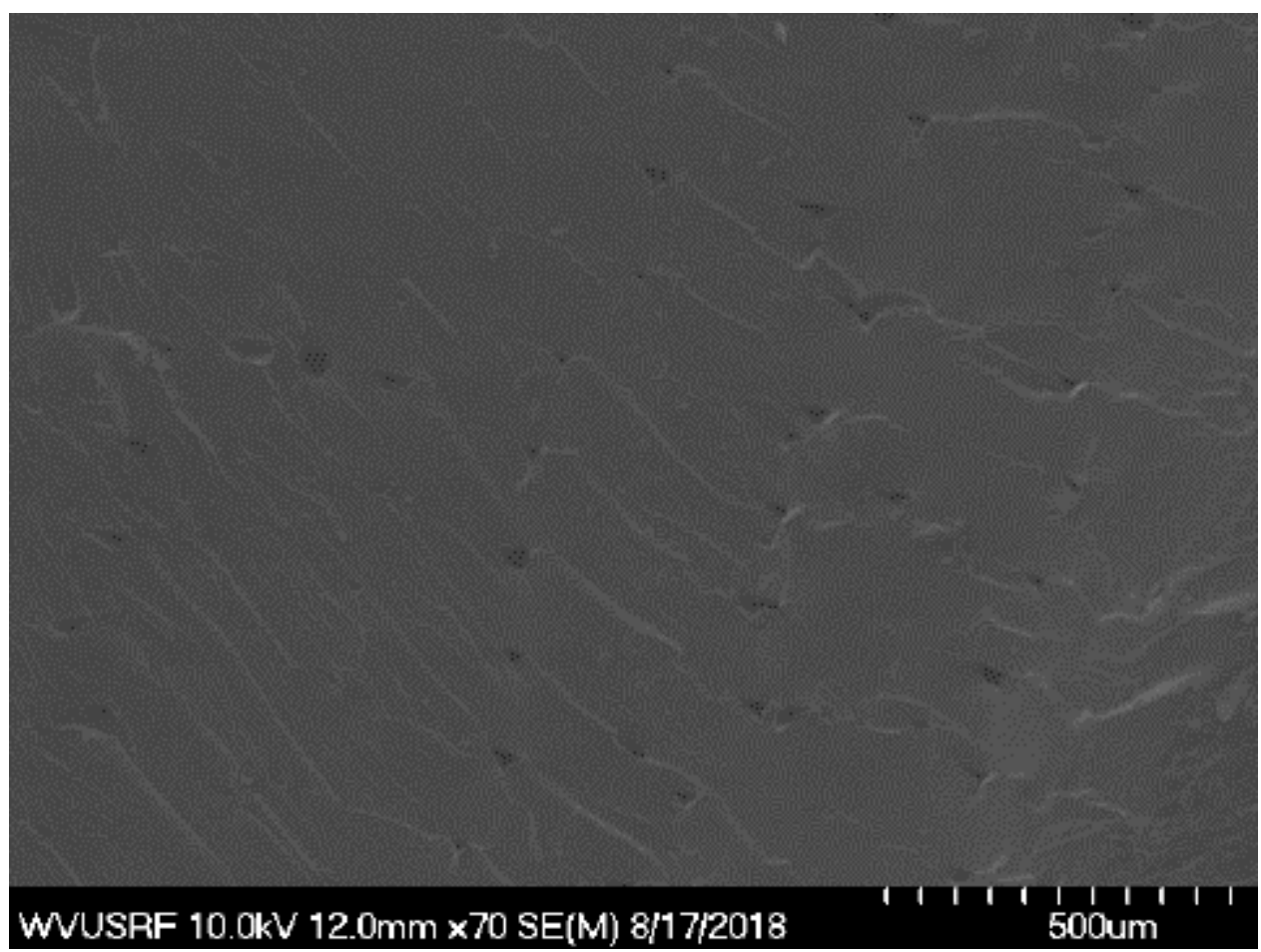

Figure 7-5 SEM image of a sample printed by $0.4 \mathrm{~mm}$ nozzle 


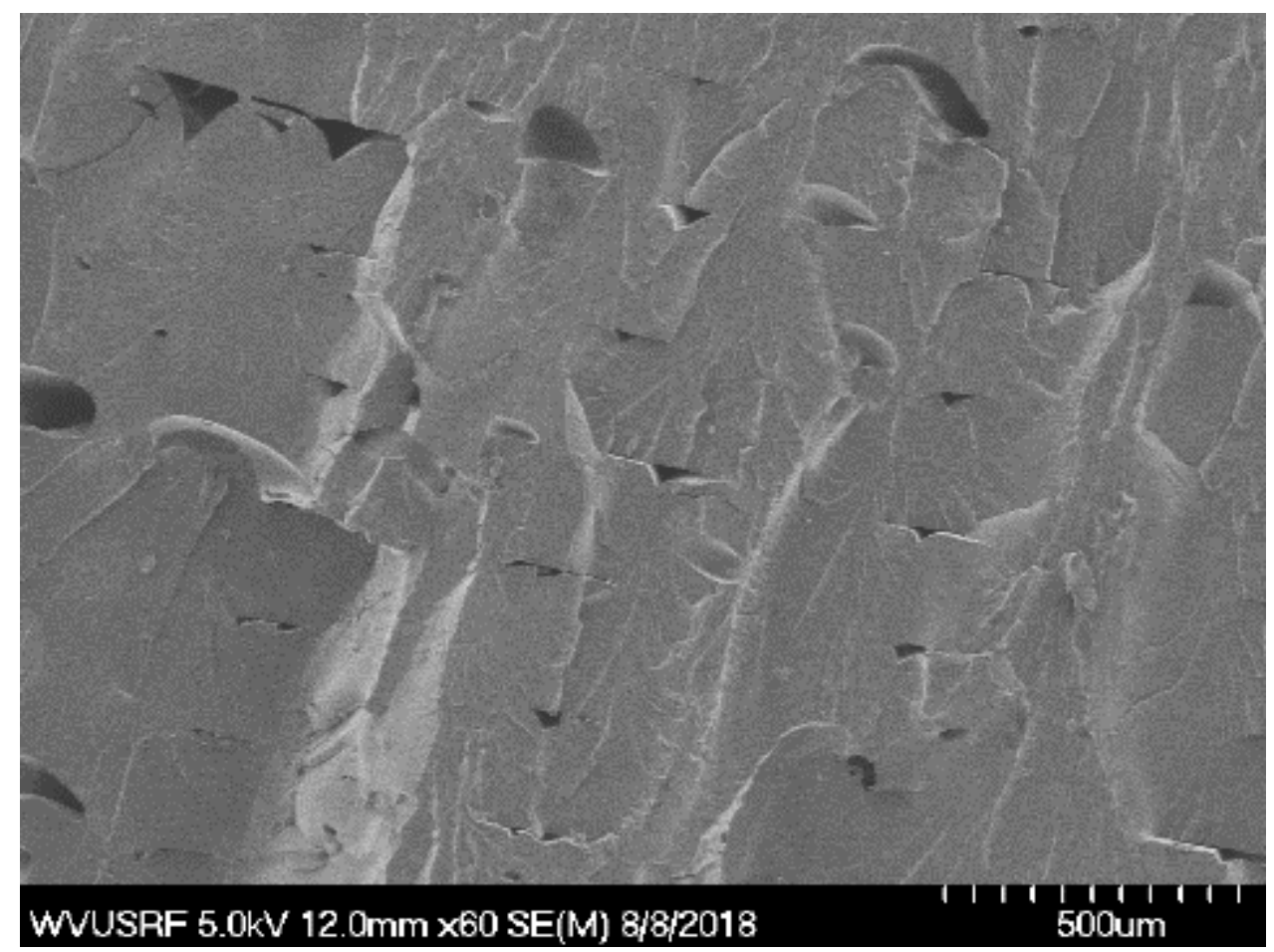

Figure 7-6 SEM image of a sample printed by $0.6 \mathrm{~mm}$ nozzle

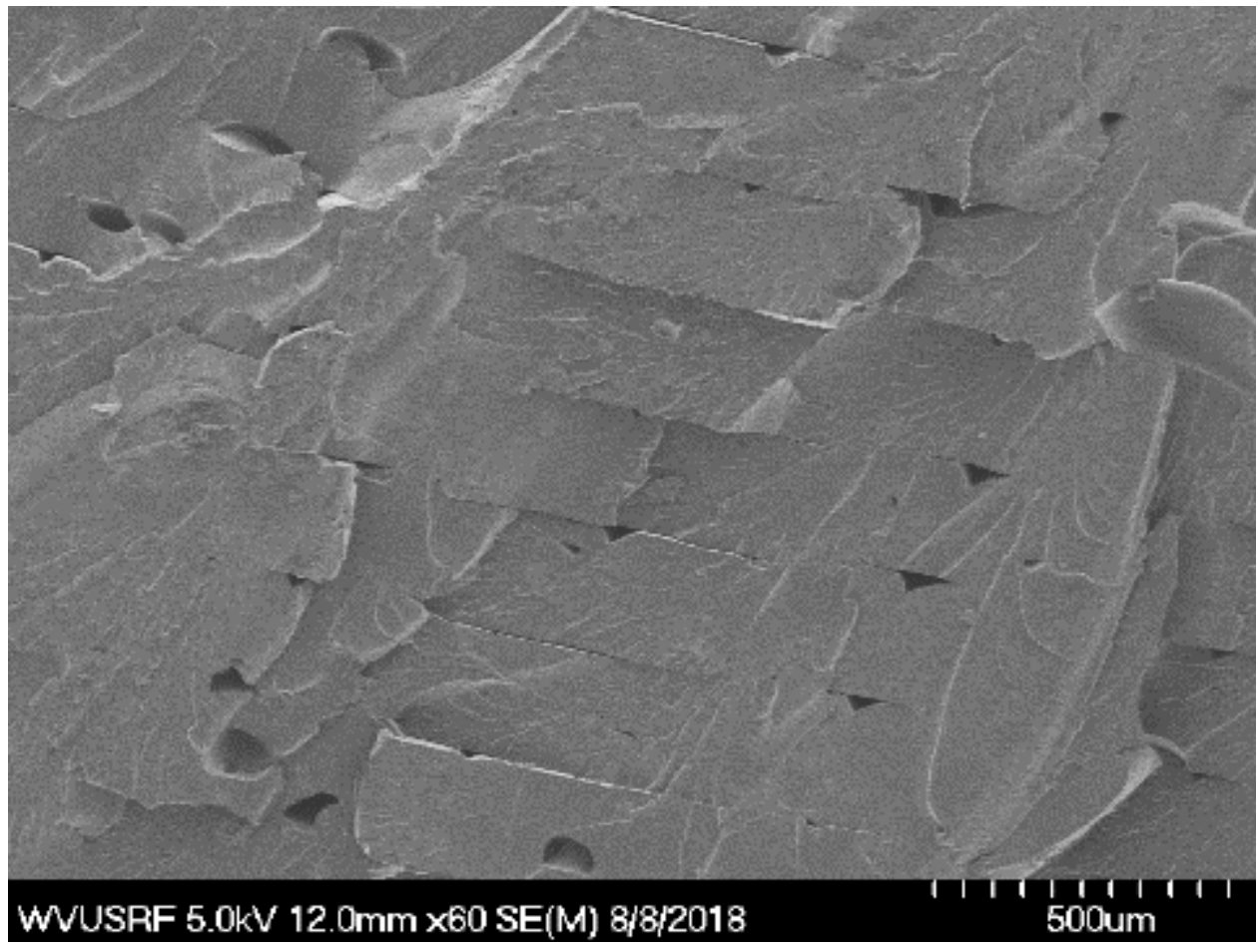

Figure 7-7 SEM image of a sample printed by $0.6 \mathrm{~mm}$ nozzle 


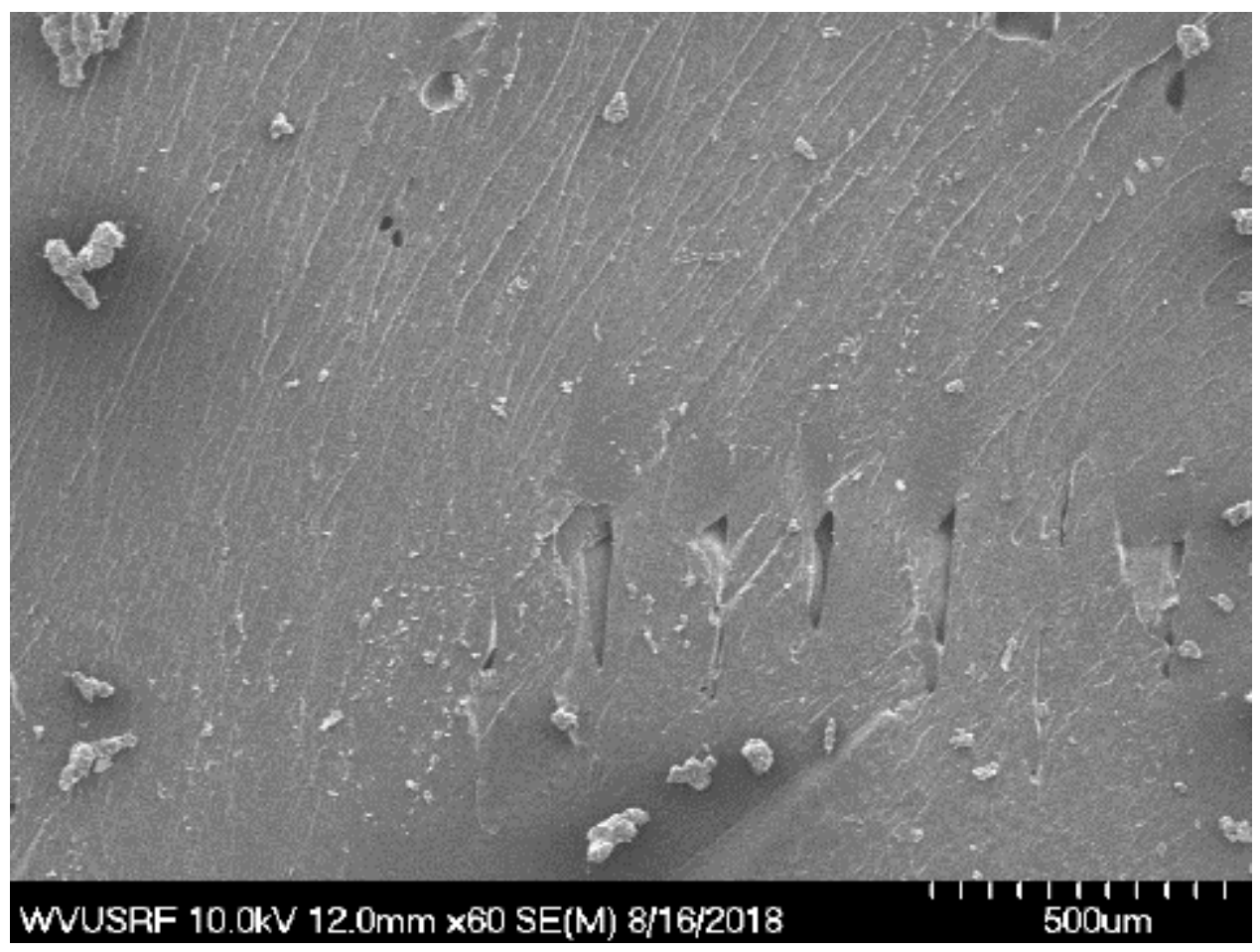

Figure 7-8 SEM image of a sample printed by $0.8 \mathrm{~mm}$ nozzle

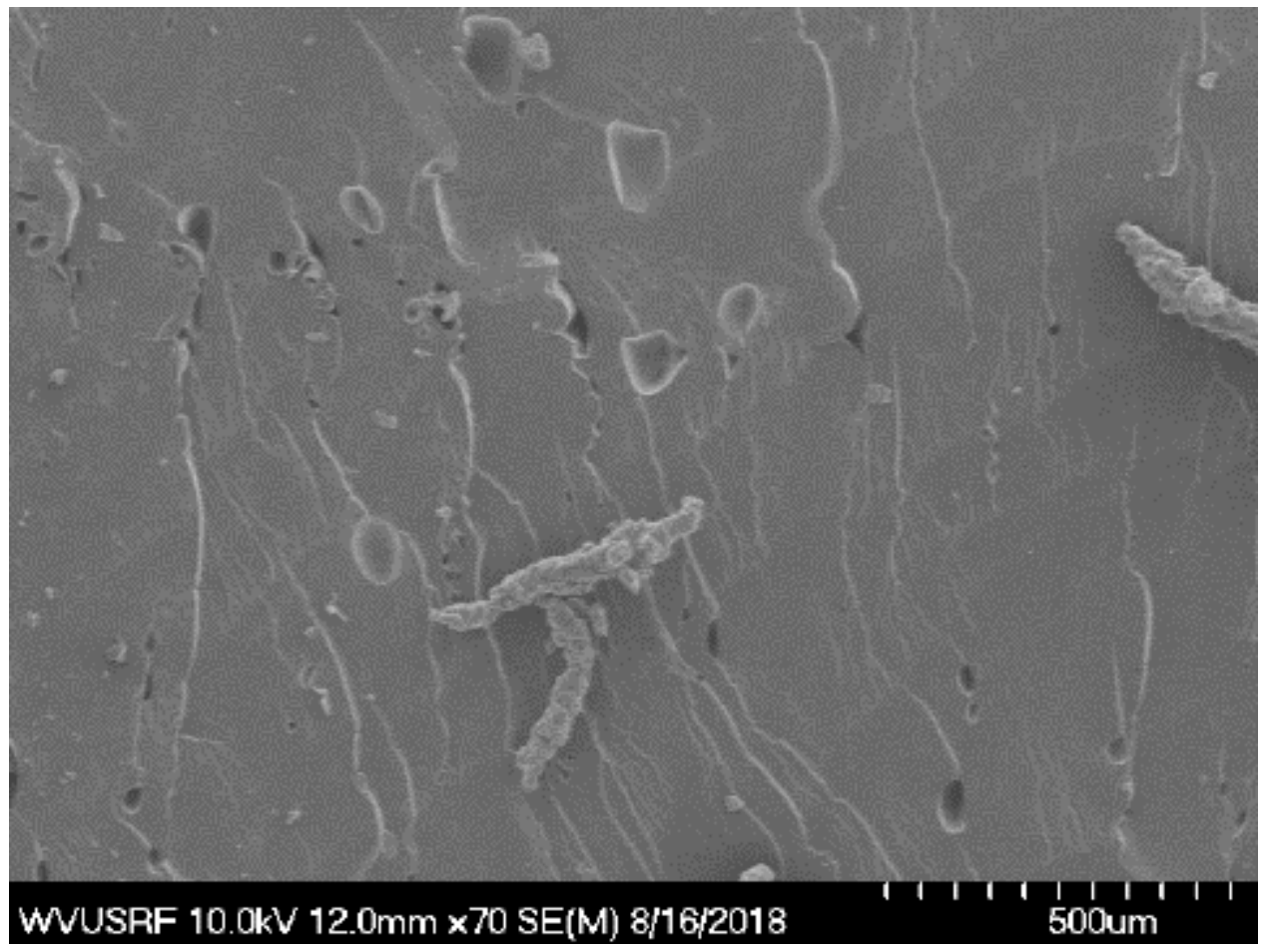

Figure 7-9 SEM image of a sample printed by $0.8 \mathrm{~mm}$ nozzle 


\section{F. Matlab codes - Fiber orientation tensor computation}

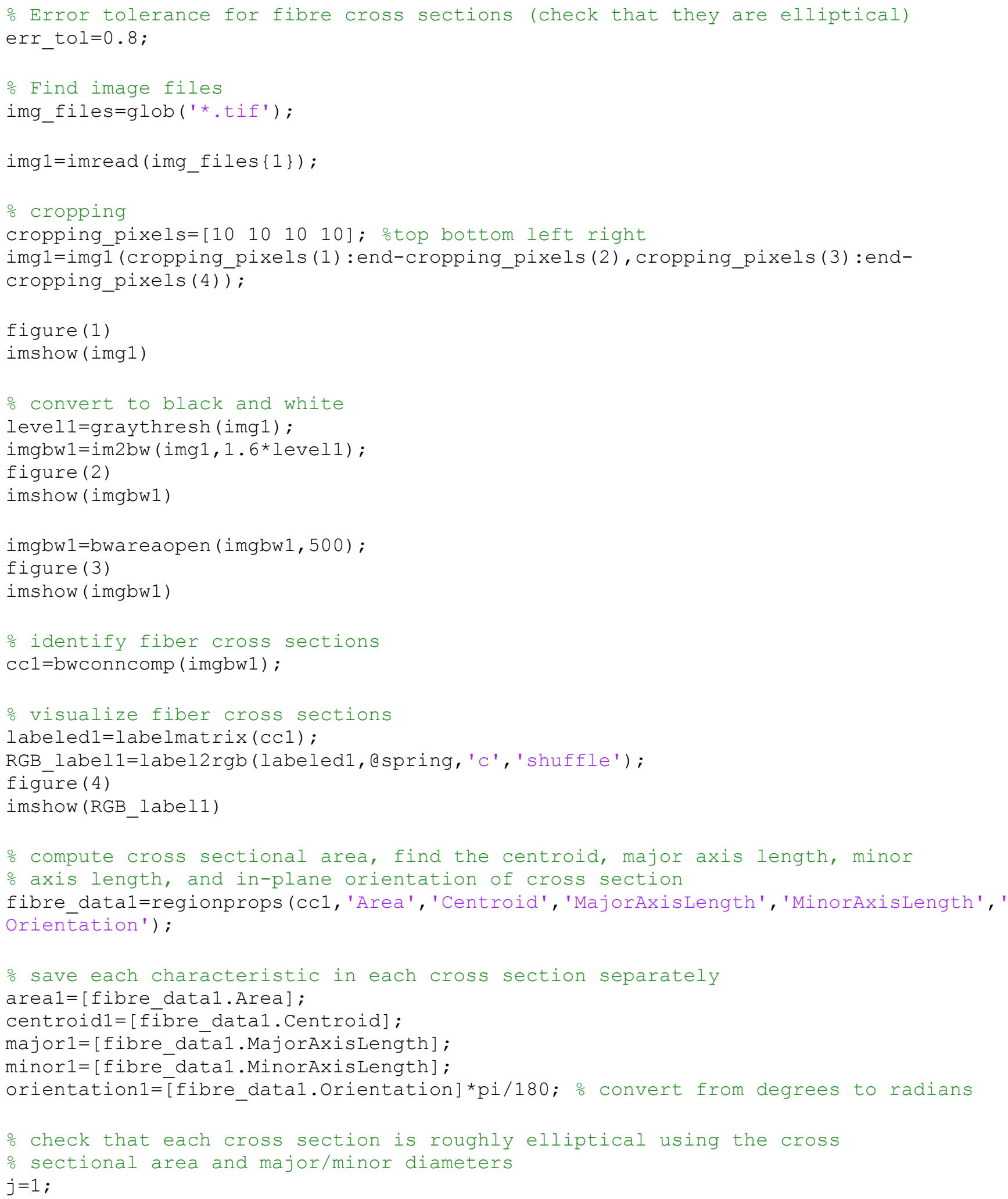




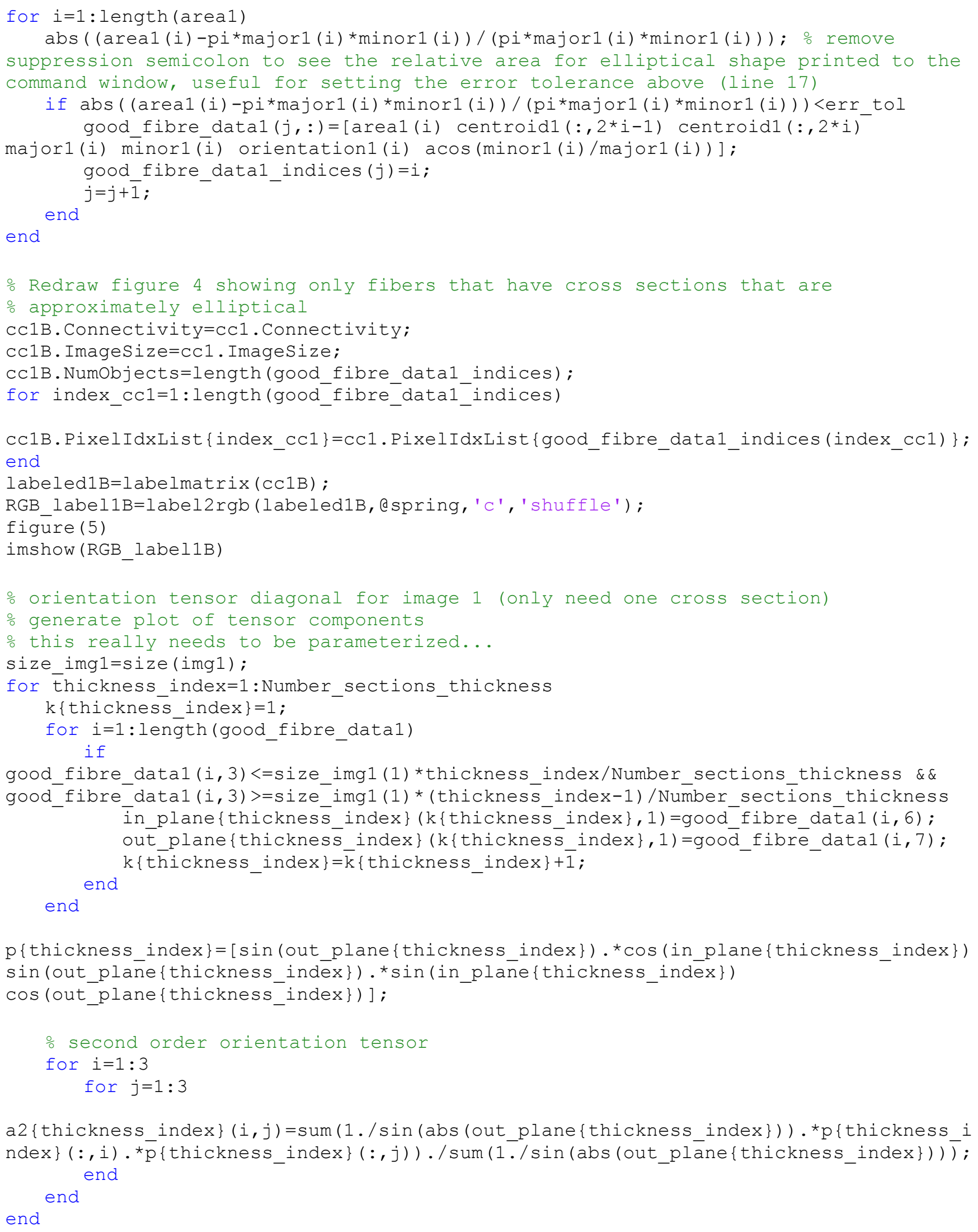




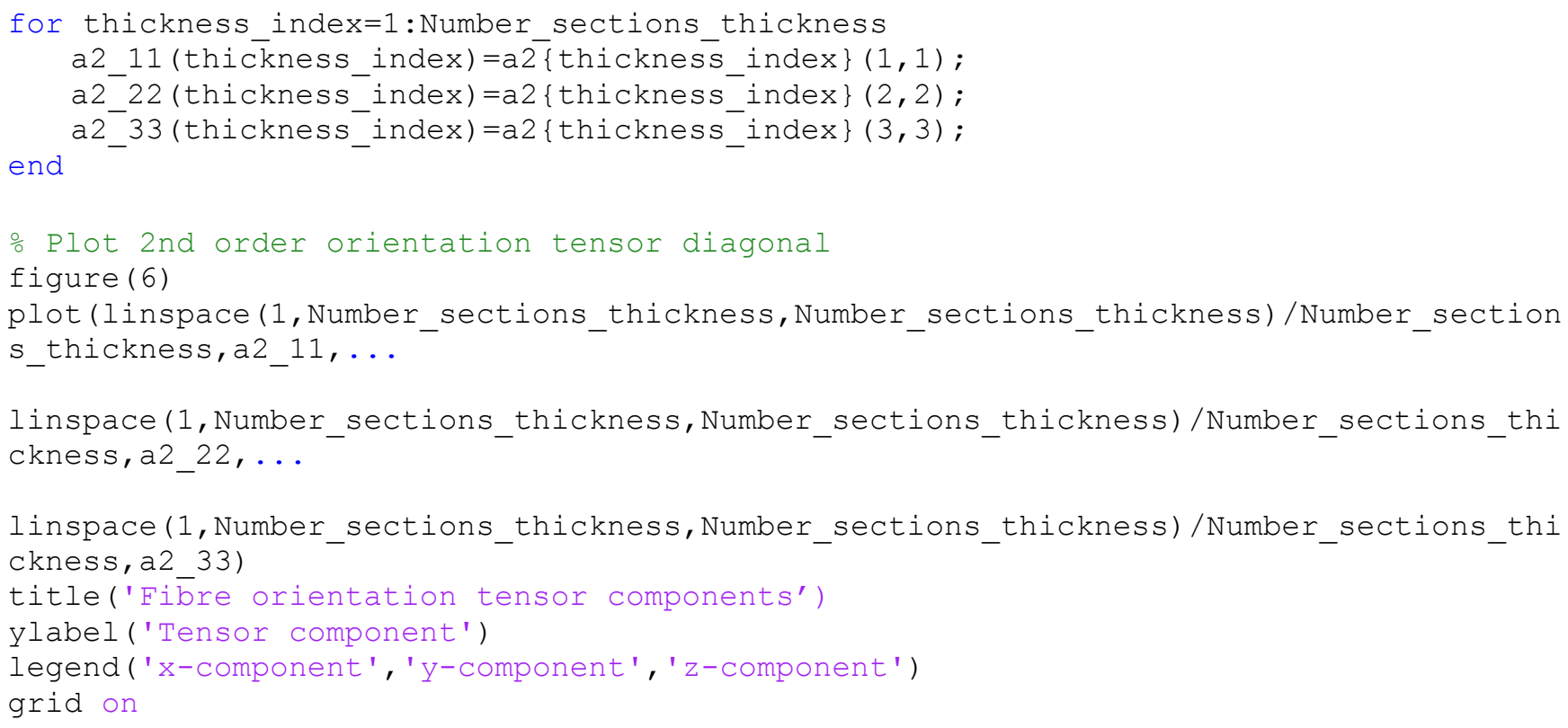



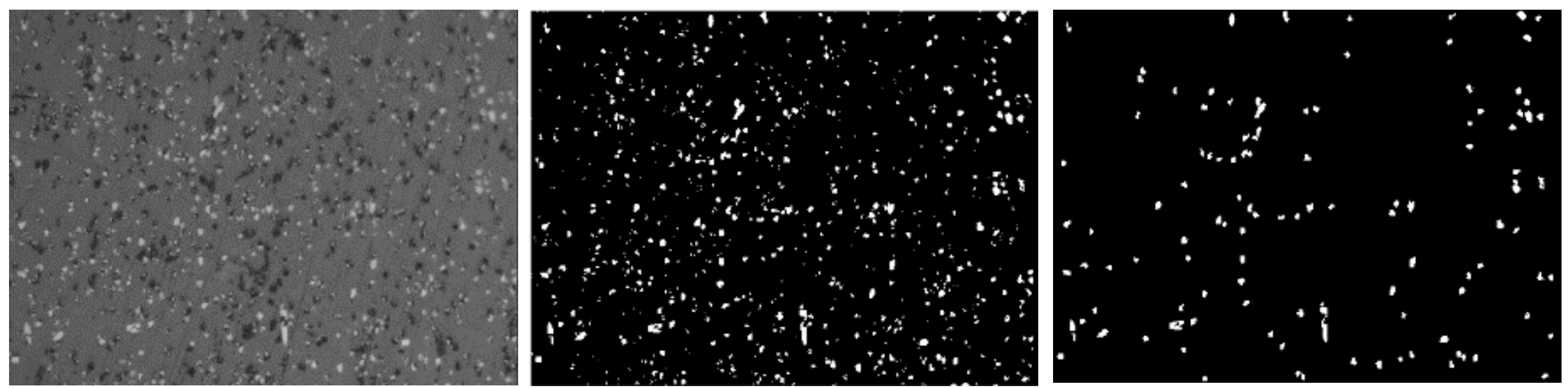

Figure 7-10 Fiber orientation computation process of a microscope image (cross-section of $0^{\circ}$ direction, 20x)
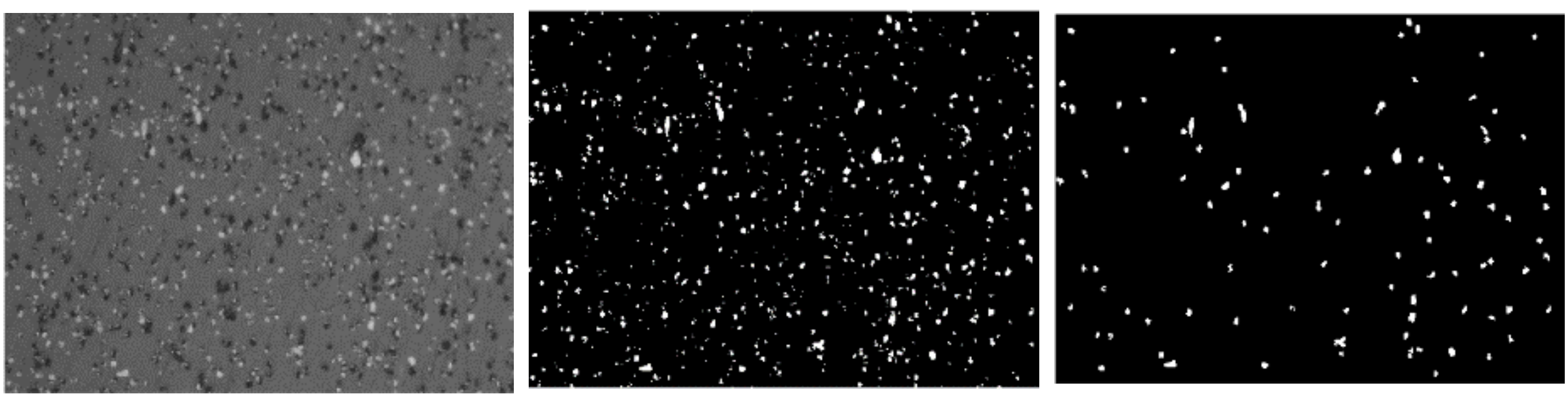

Figure 7-11 Fiber orientation computation process of a microscope image (cross-section of $0^{\circ}$ direction, 20x) 

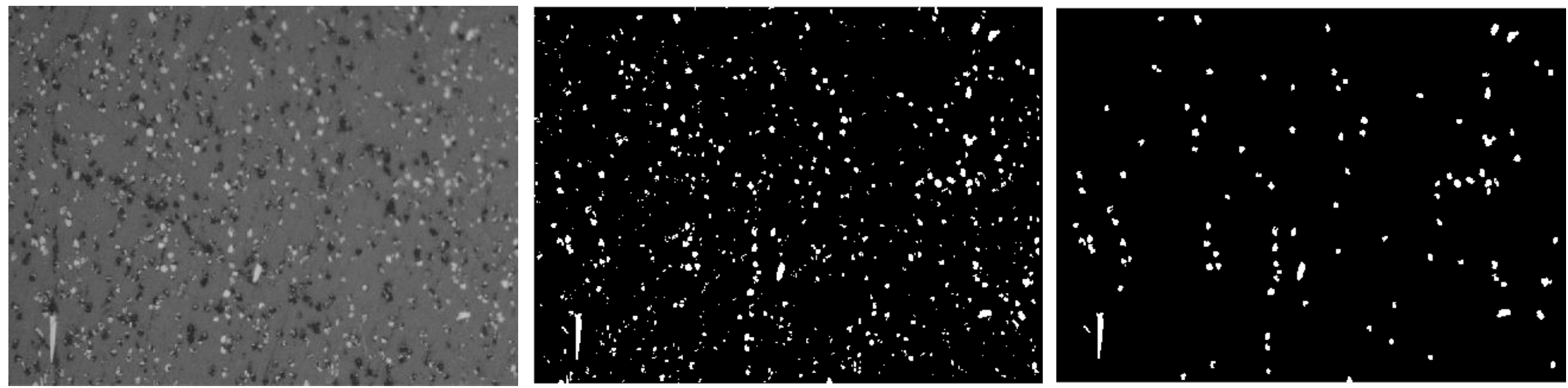

Figure 7-12 Fiber orientation computation process of a microscope image (cross-section of $0^{\circ}$ direction, 20x)
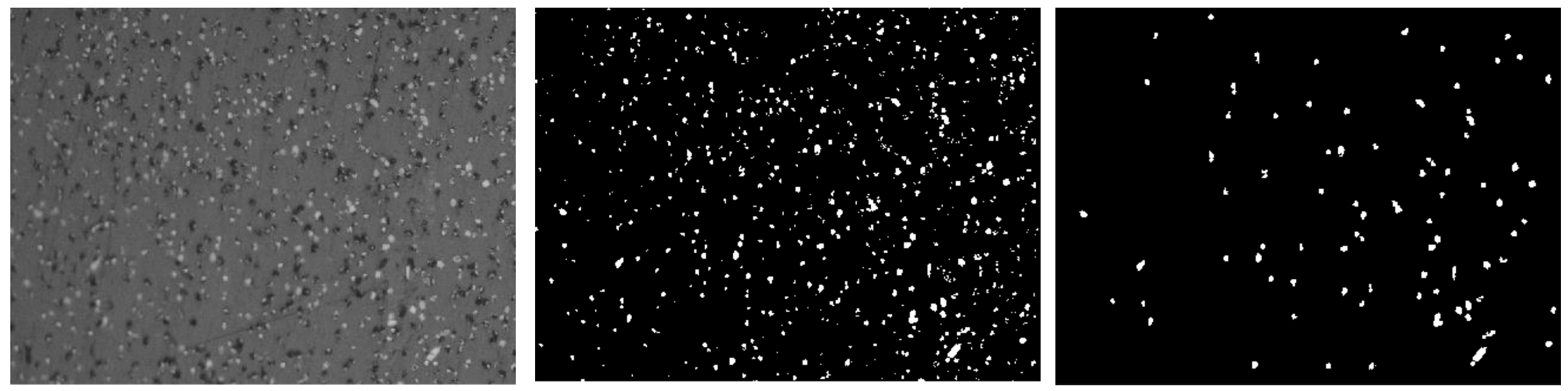

Figure 7-13 Fiber orientation computation process of a microscope image (cross-section of $0^{\circ}$ direction, 20x) 

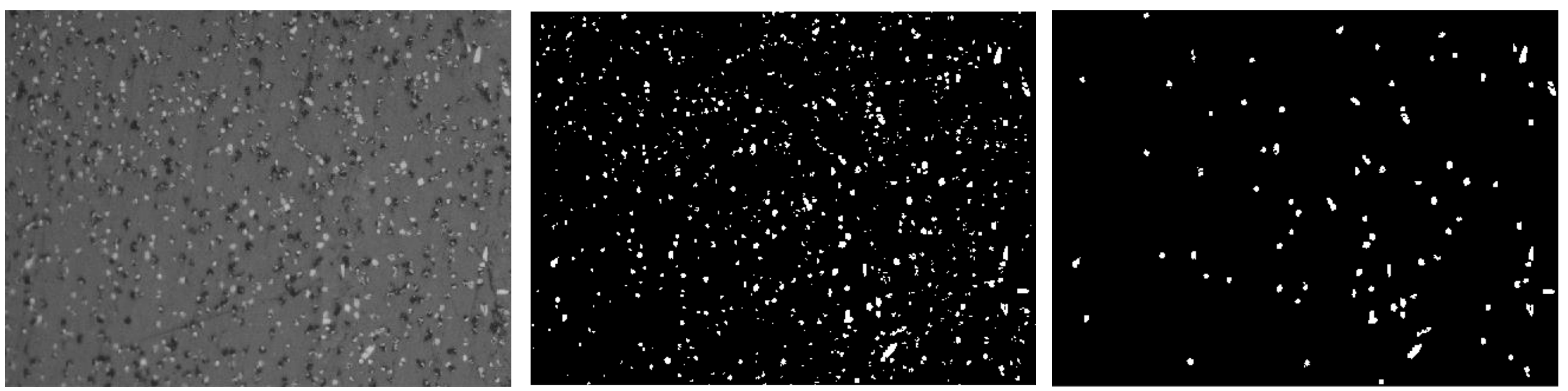

Figure 7-14 Fiber orientation computation process of a microscope image (cross-section of $0^{\circ}$ direction, 20x)
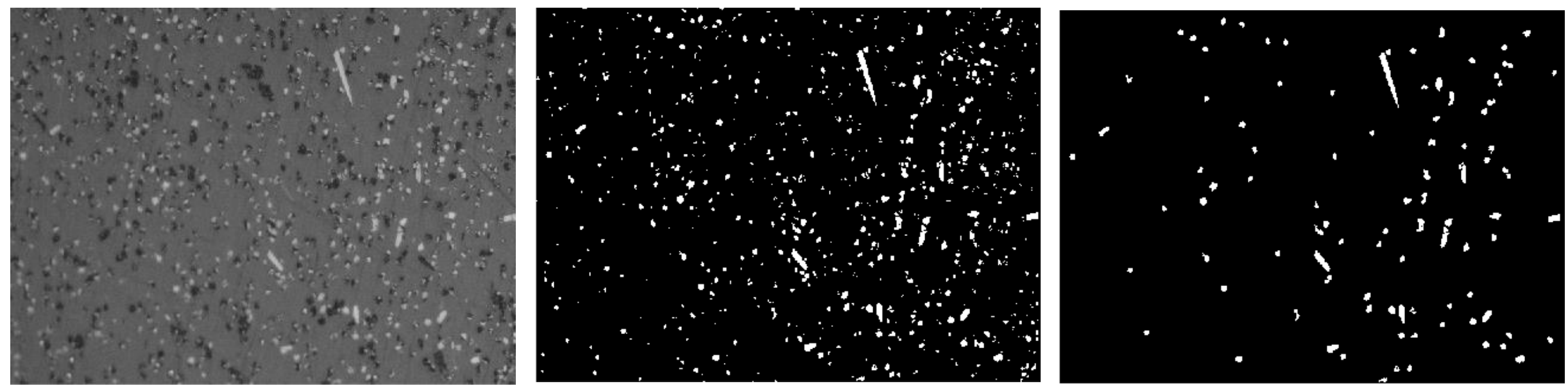

Figure 7-15 Fiber orientation computation process of a microscope image (cross-section of $0^{\circ}$ direction, 20x) 

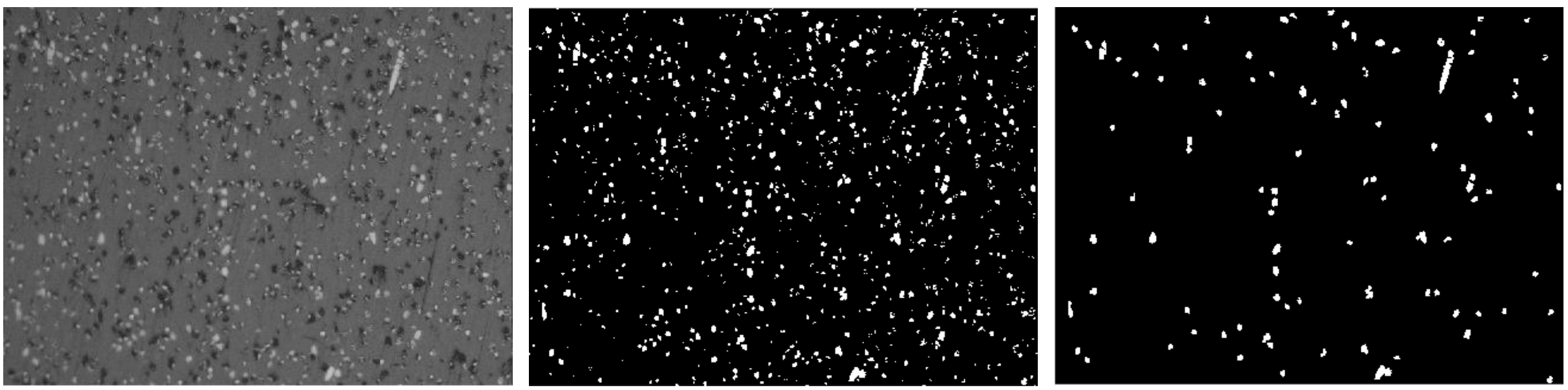

Figure 7-16 Fiber orientation computation process of a microscope image (cross-section of $0^{\circ}$ direction, 20x)
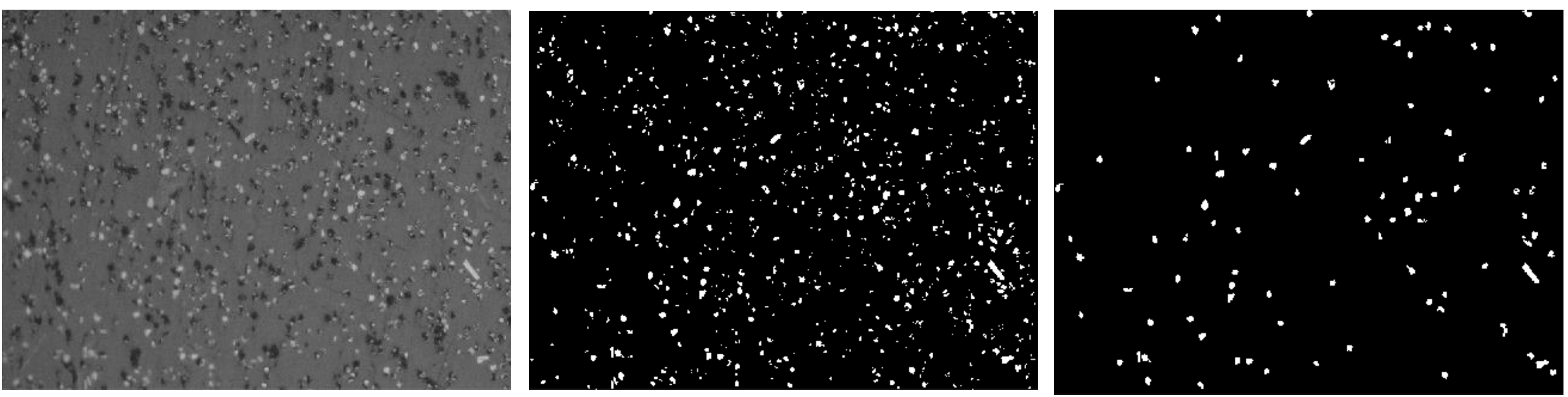

Figure 7-17 Fiber orientation computation process of a microscope image (cross-section of $0^{\circ}$ direction, 20x) 

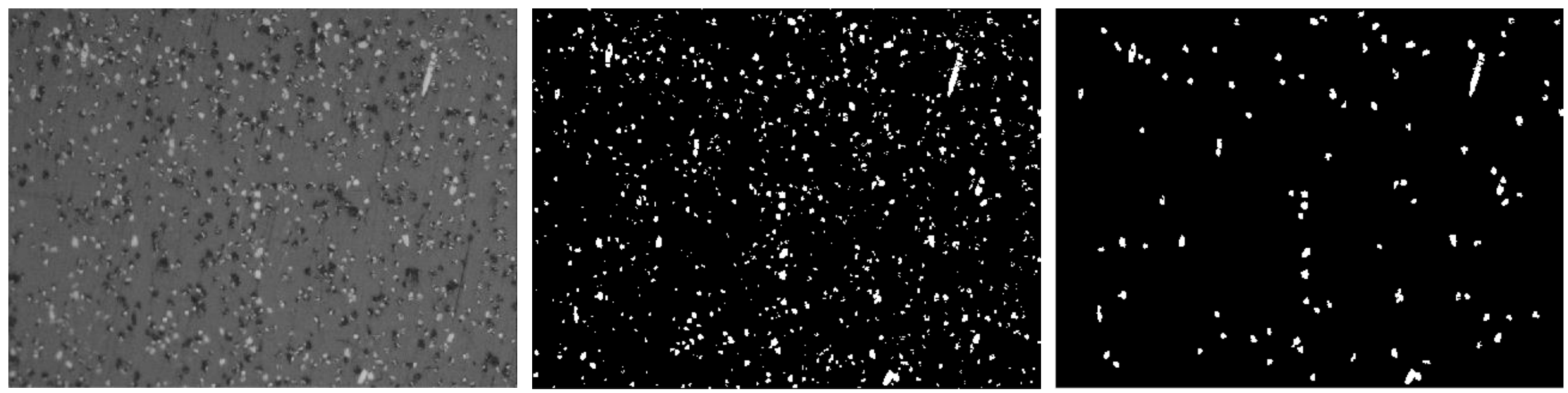

Figure 7-18 Fiber orientation computation process of a microscope image (cross-section of $0^{\circ}$ direction, 20x)
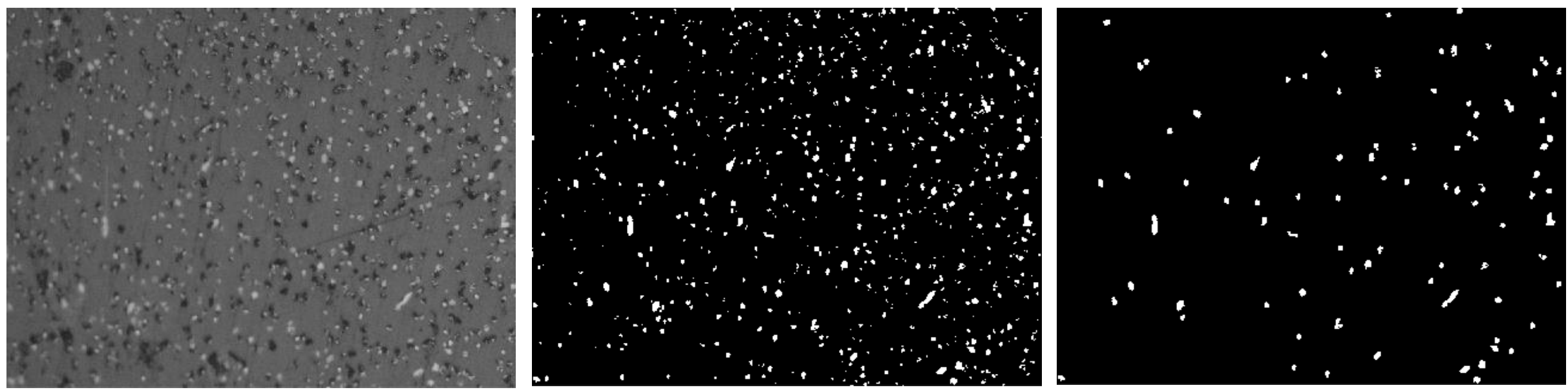

Figure 7-19 Fiber orientation computation process of a microscope image (cross-section of $0^{\circ}$ direction, 20x) 

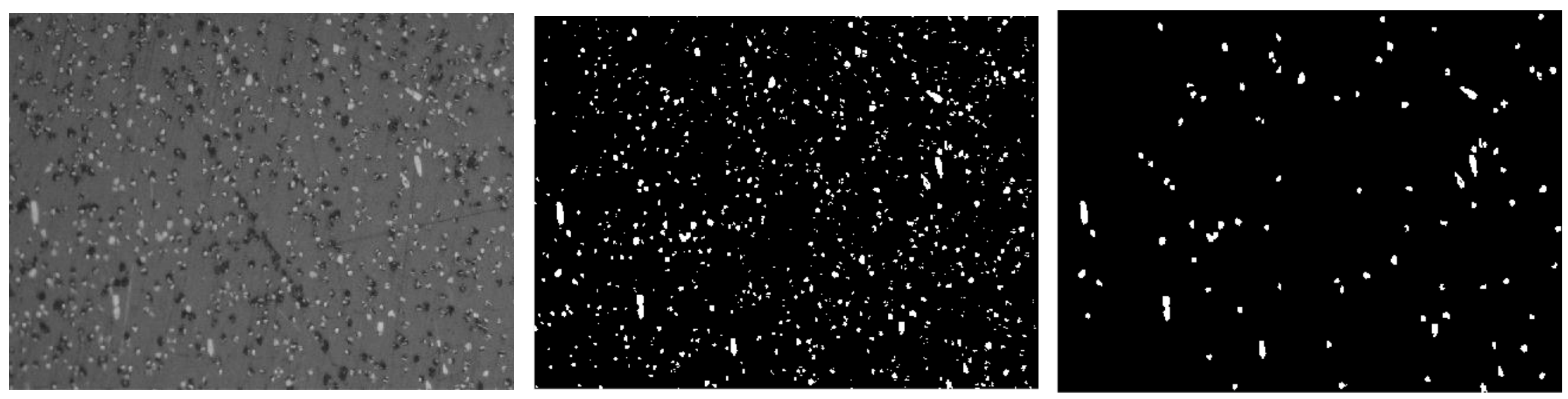

Figure 7-20 Fiber orientation computation process of a microscope image (cross-section of $0^{\circ}$ direction, 20x)
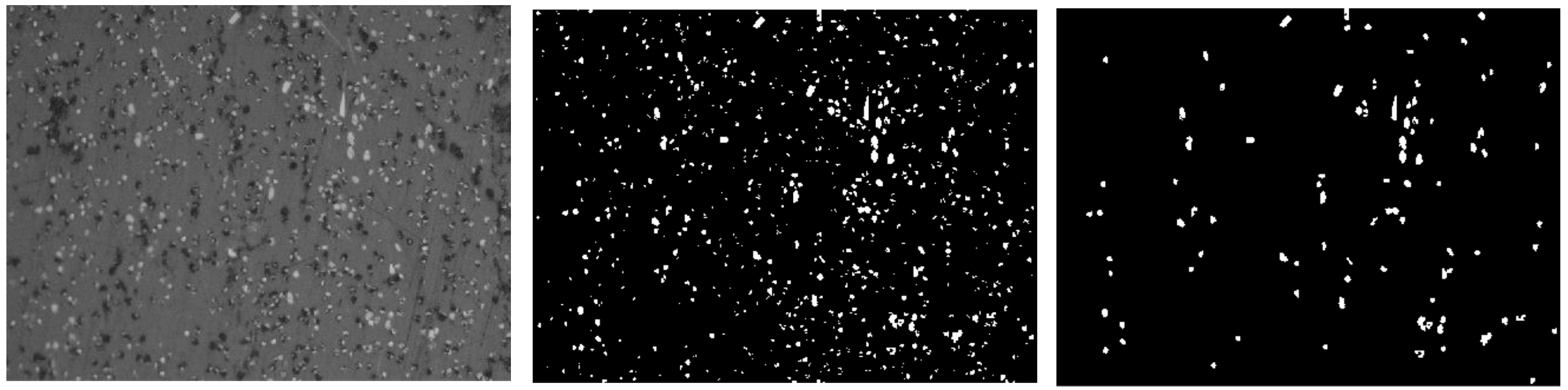

Figure 7-21 Fiber orientation computation process of a microscope image (cross-section of $0^{\circ}$ direction, 20x) 

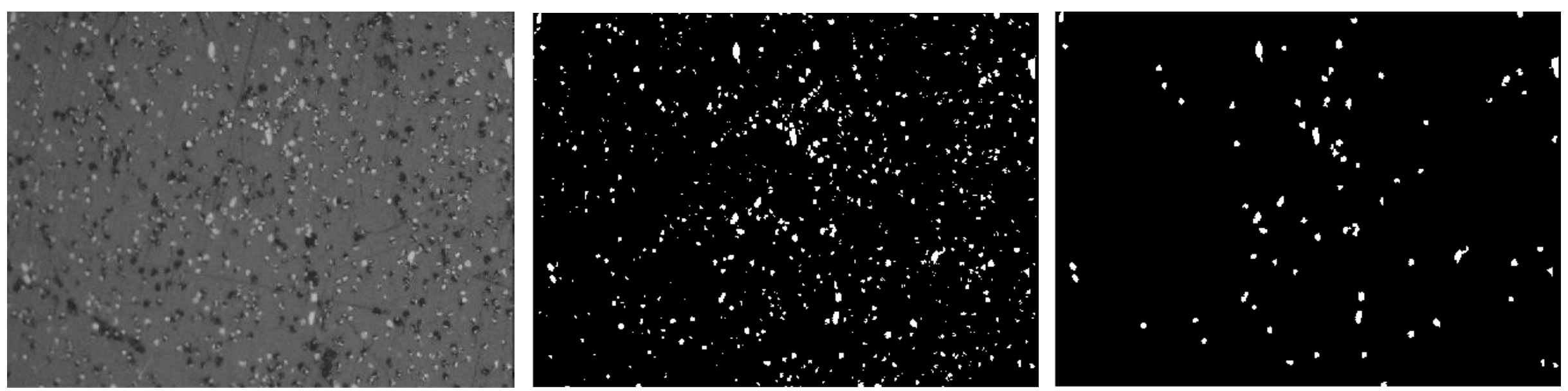

Figure 7-22 Fiber orientation computation process of a microscope image (cross-section of $0^{\circ}$ direction, 20x)
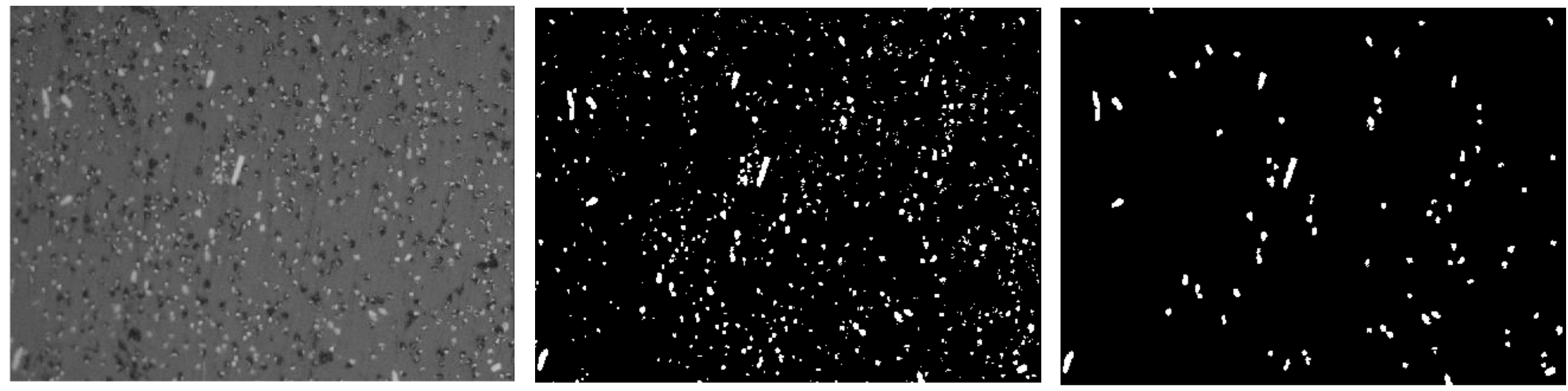

Figure 7-23 Fiber orientation computation process of a microscope image (cross-section of $0^{\circ}$ direction, 20x) 

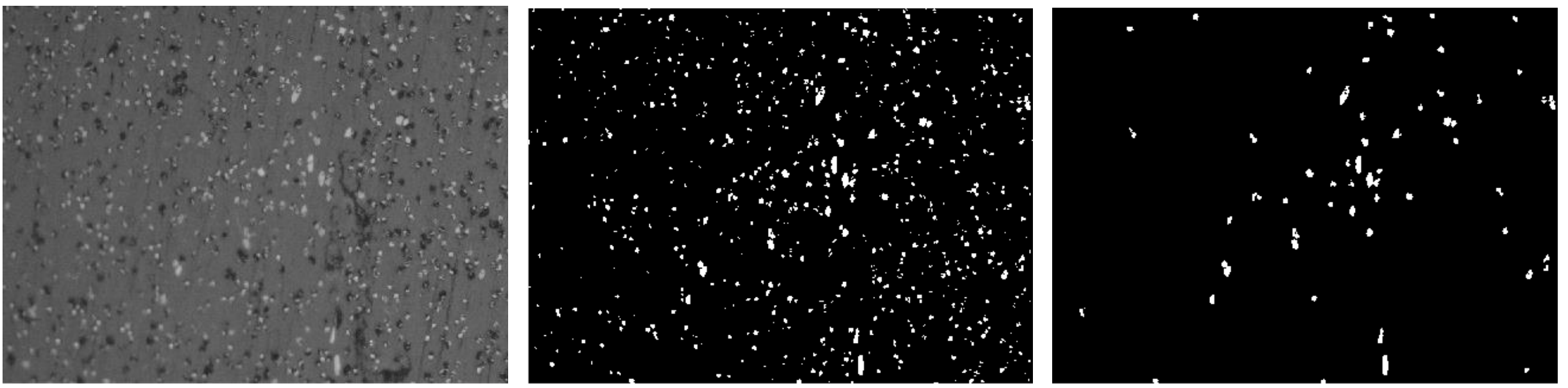

Figure 7-24 Fiber orientation computation process of a microscope image (cross-section of $0^{\circ}$ direction, 20x)
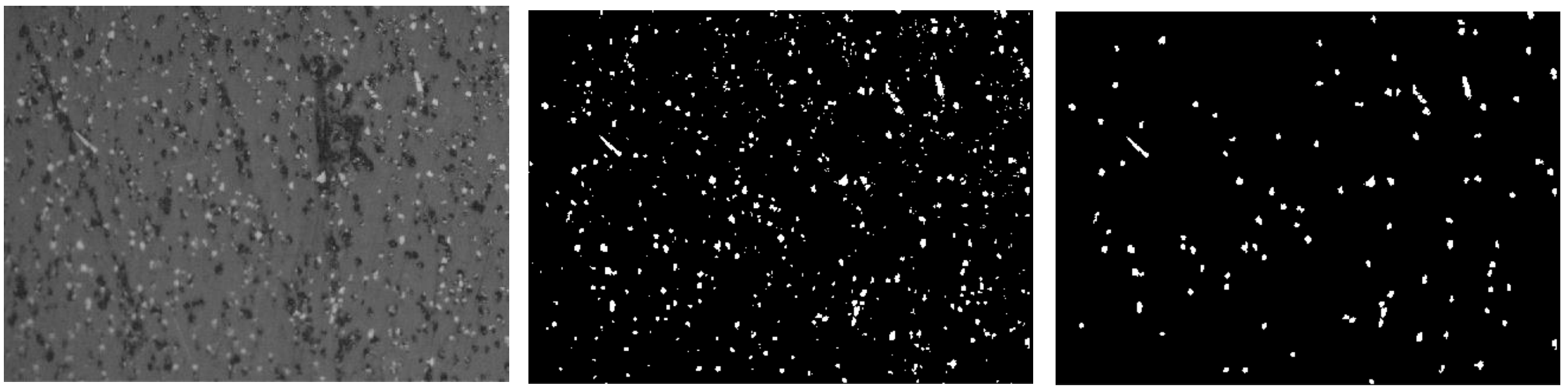

Figure 7-25 Fiber orientation computation process of a microscope image (cross-section of $0^{\circ}$ direction, 20x) 

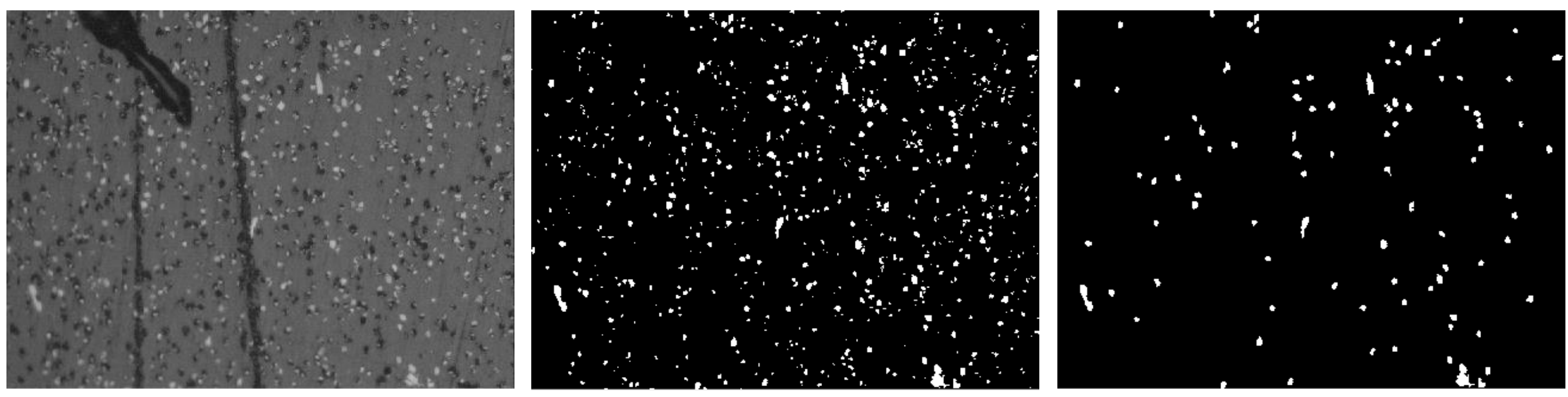

Figure 7-26 Fiber orientation computation process of a microscope image (cross-section of $0^{\circ}$ direction, 20x)
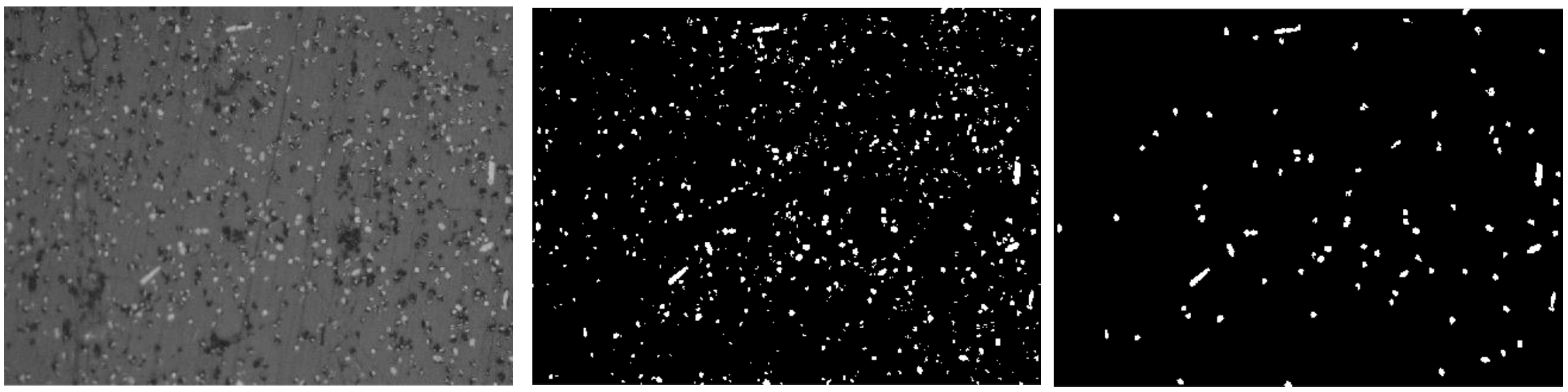

Figure 7-27 Fiber orientation computation process of a microscope image (cross-section of $0^{\circ}$ direction, 20x) 

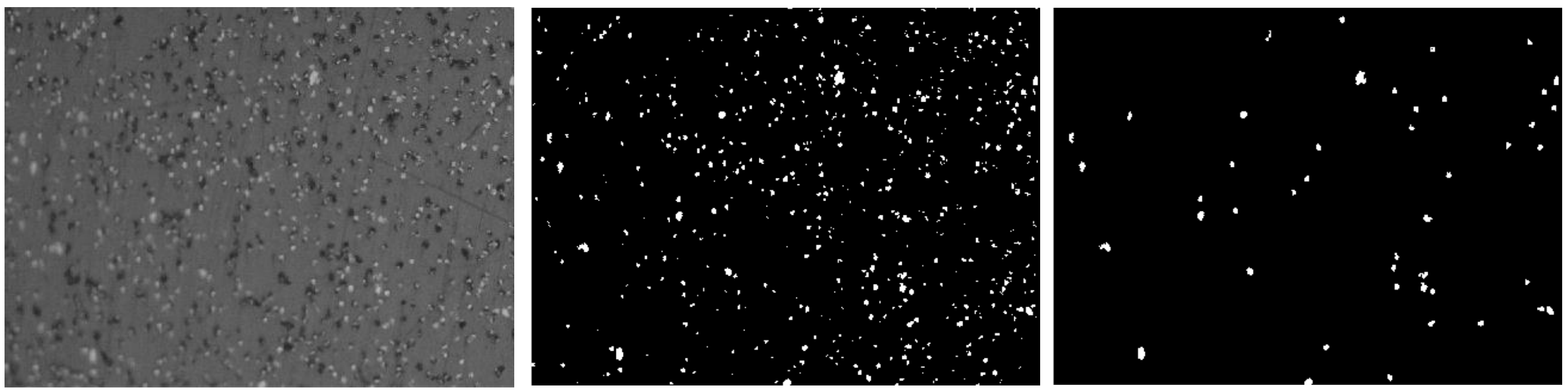

Figure 7-28 Fiber orientation computation process of a microscope image (cross-section of $0^{\circ}$ direction, 20x)
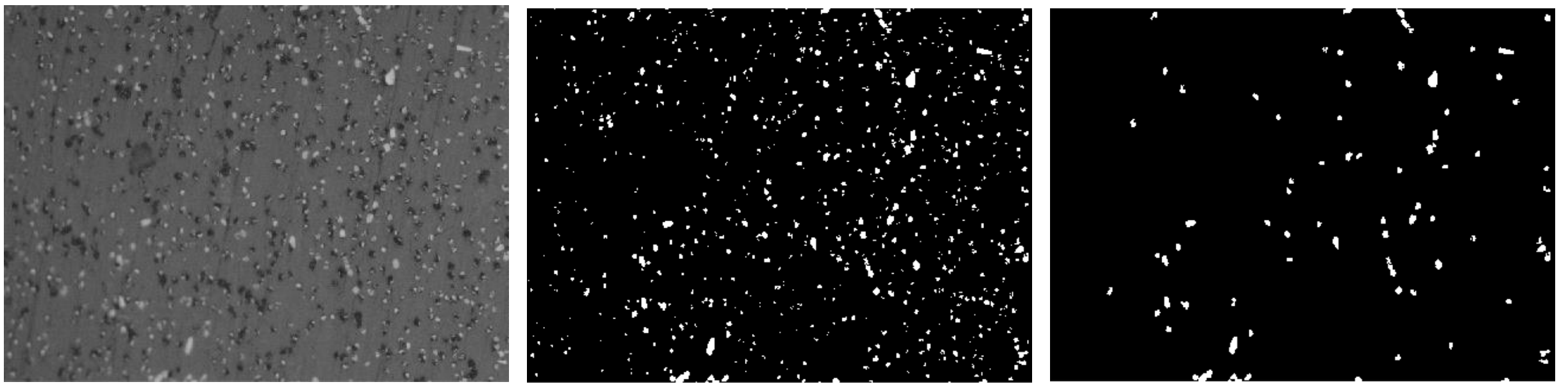

Figure 7-29 Fiber orientation computation process of a microscope image (cross-section of $0^{\circ}$ direction, 20x) 

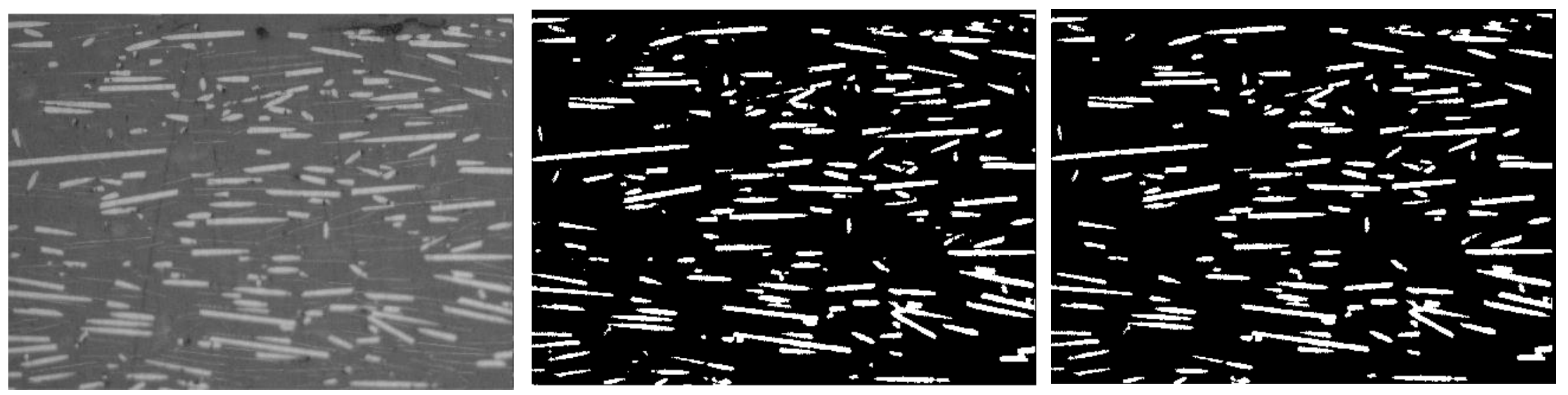

Figure 7-30 Fiber orientation computation process of a microscope image (cross-section of $90^{\circ}$ direction, 20x)
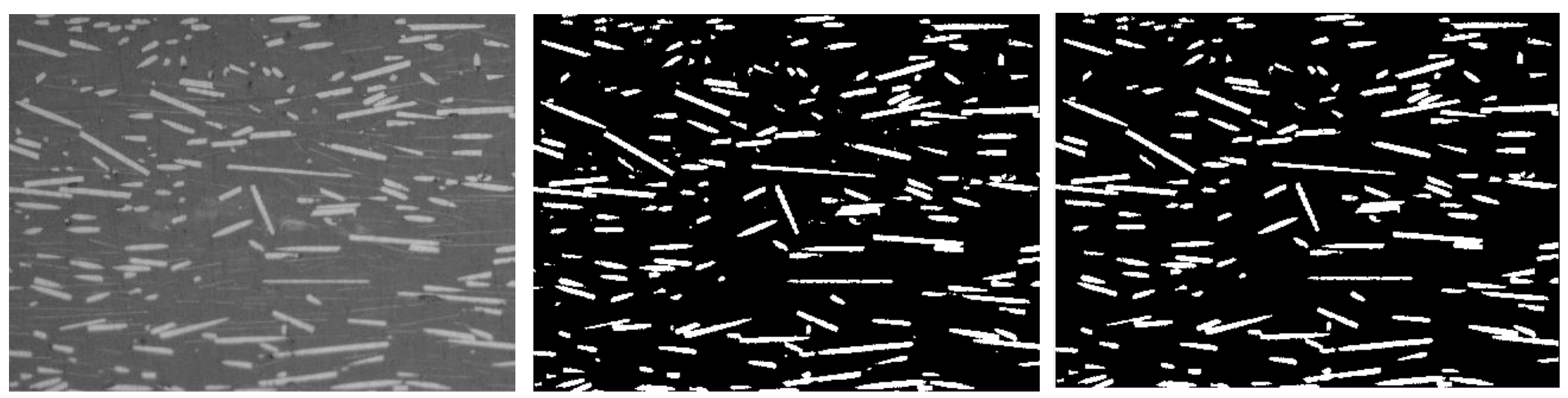

Figure 7-31 Fiber orientation computation process of a microscope image (cross-section of $90^{\circ}$ direction, 20x) 

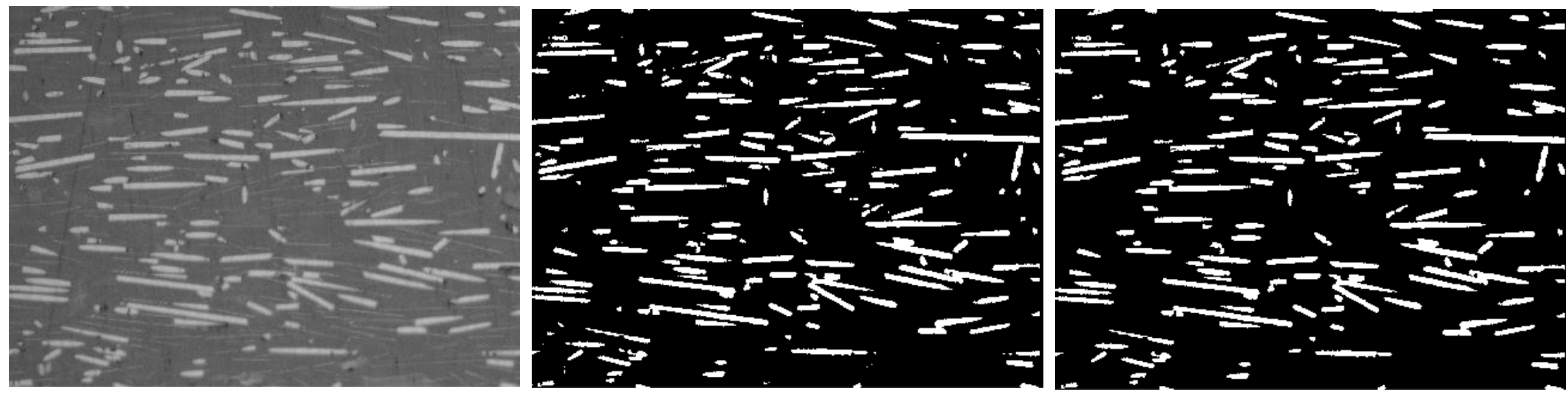

Figure 7-32 Fiber orientation computation process of a microscope image (cross-section of $90^{\circ}$ direction, 20x)
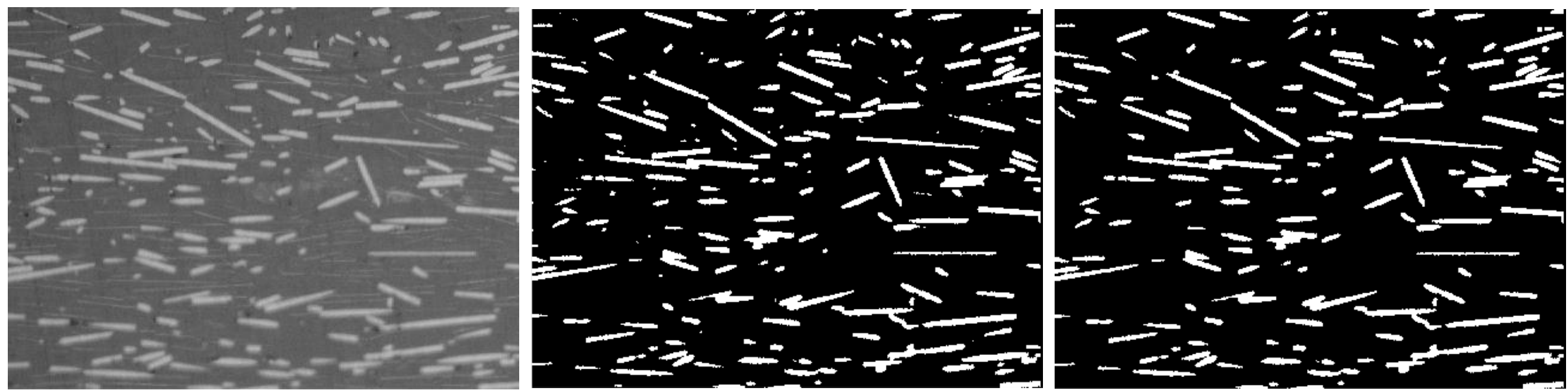

Figure 7-33 Fiber orientation computation process of a microscope image (cross-section of $90^{\circ}$ direction, 20x) 

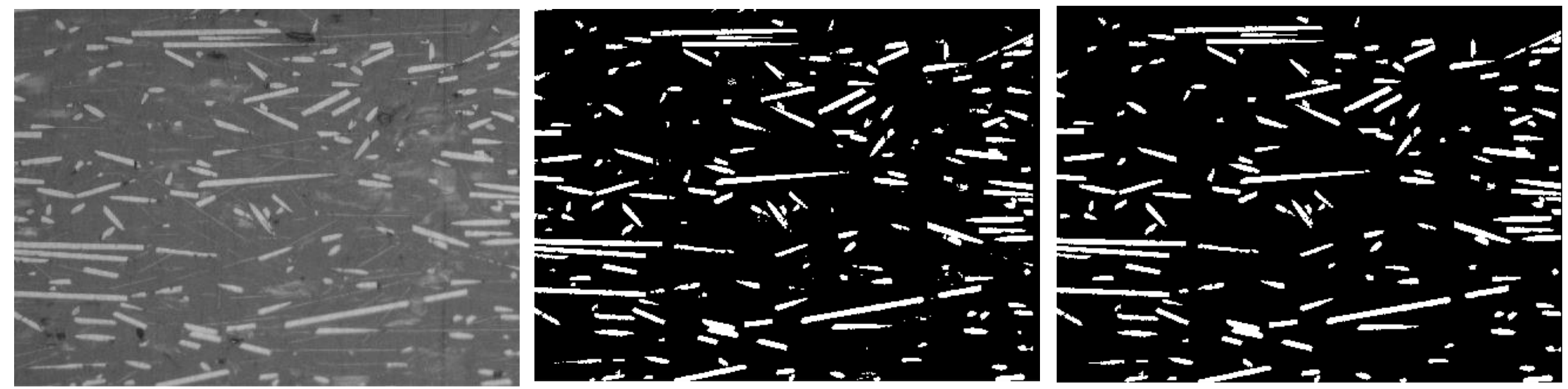

Figure 7-34 Fiber orientation computation process of a microscope image (cross-section of $90^{\circ}$ direction, 20x)
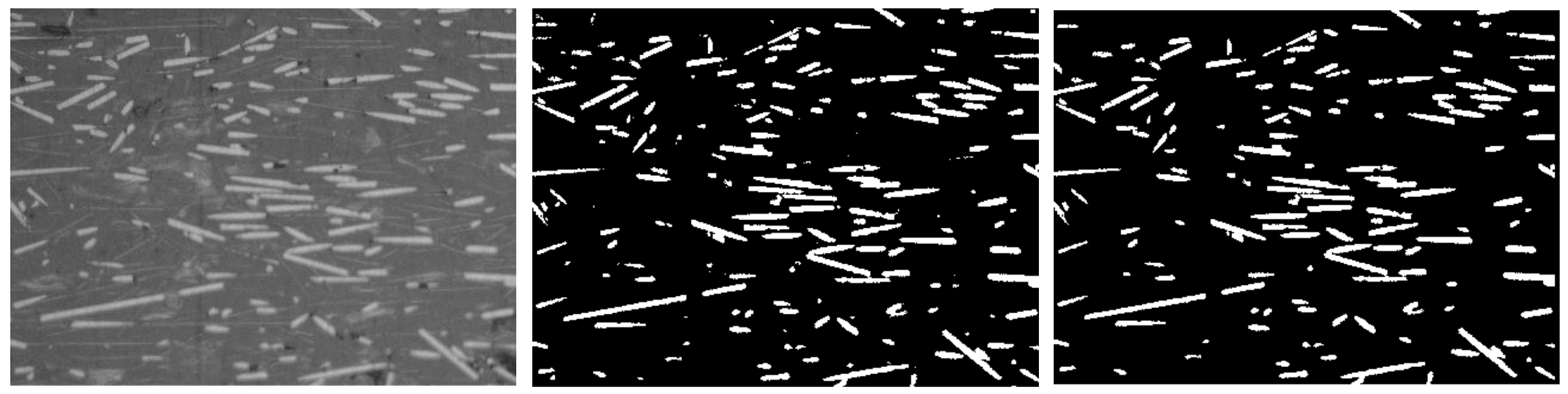

Figure 7-35 Fiber orientation computation process of a microscope image (cross-section of $90^{\circ}$ direction, 20x) 

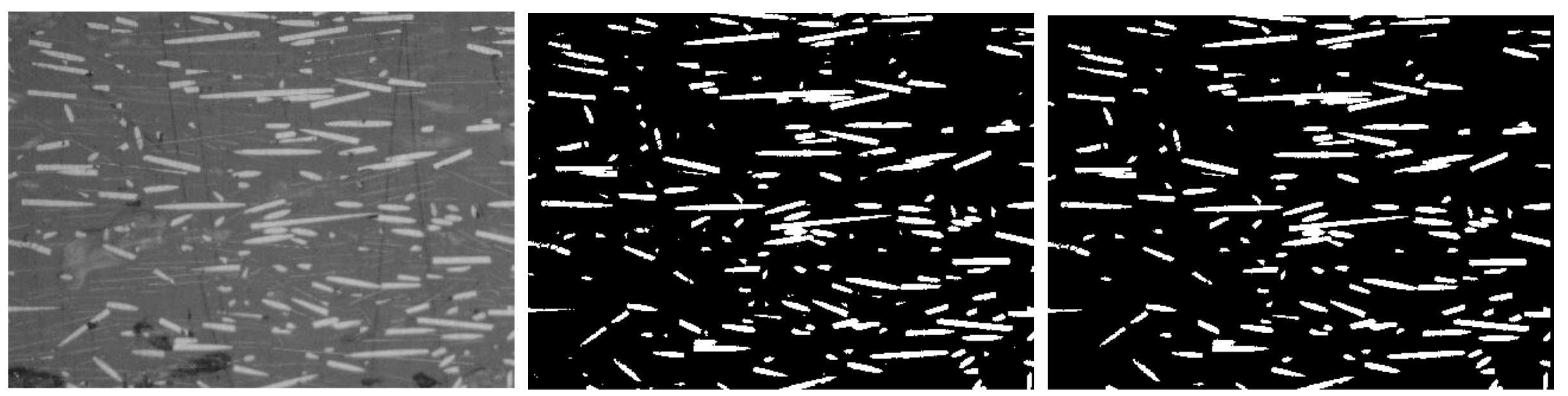

Figure 7-36 Fiber orientation computation process of a microscope image (cross-section of $90^{\circ}$ direction, 20x)
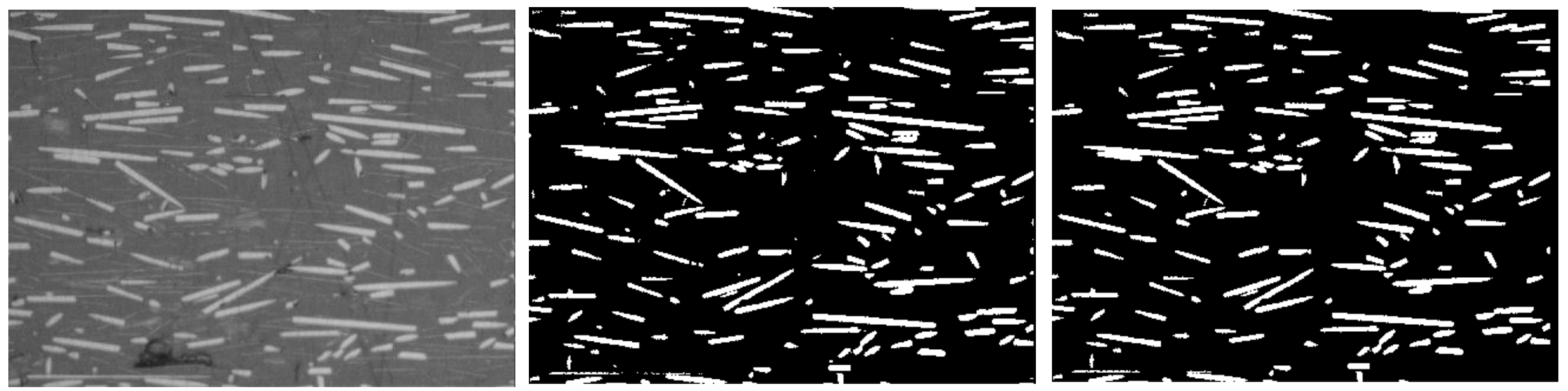

Figure 7-37 Fiber orientation computation process of a microscope image (cross-section of $90^{\circ}$ direction, 20x) 

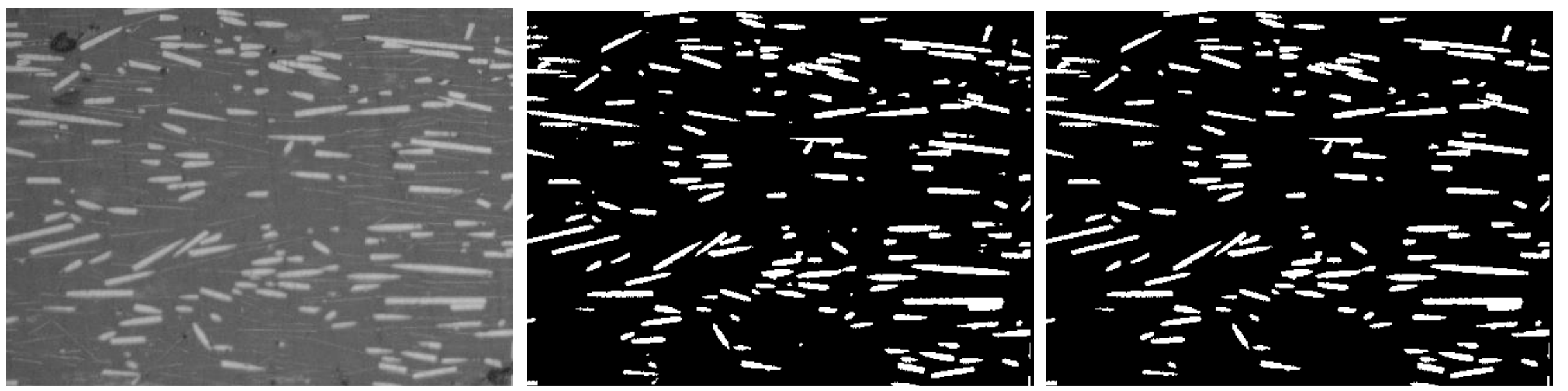

Figure 7-38 Fiber orientation computation process of a microscope image (cross-section of $90^{\circ}$ direction, 20x)
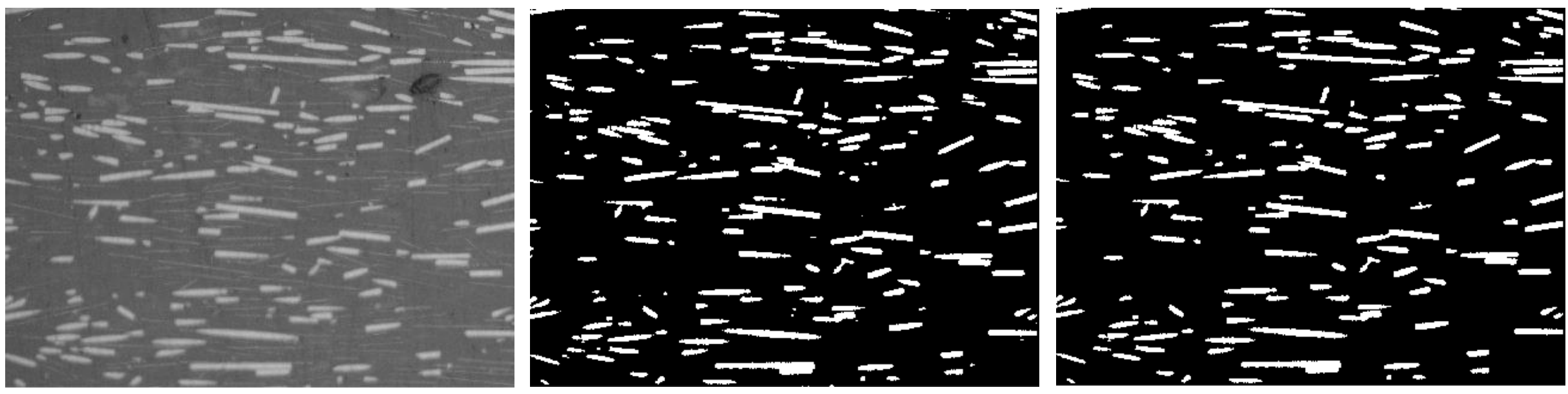

Figure 7-39 Fiber orientation computation process of a microscope image (cross-section of $90^{\circ}$ direction, 20x) 

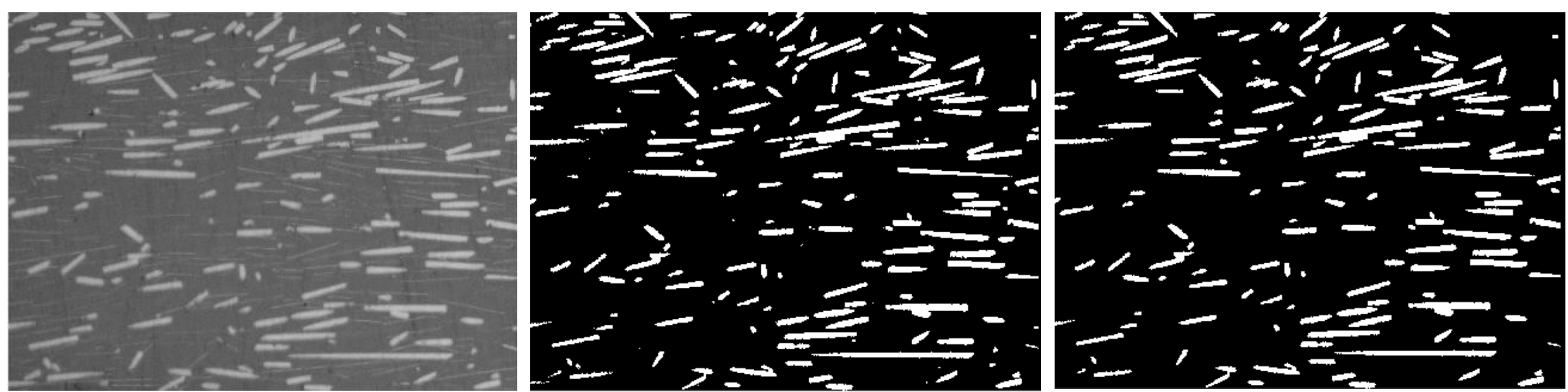

Figure 7-40 Fiber orientation computation process of a microscope image (cross-section of $90^{\circ}$ direction, 20x)
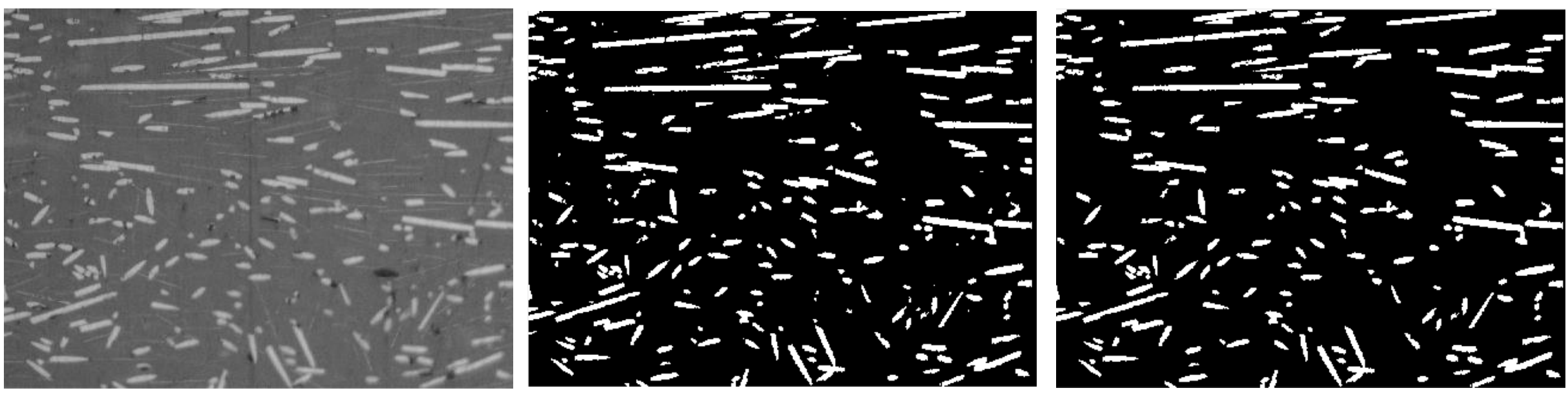

Figure 7-41 Fiber orientation computation process of a microscope image (cross-section of $90^{\circ}$ direction, 20x) 


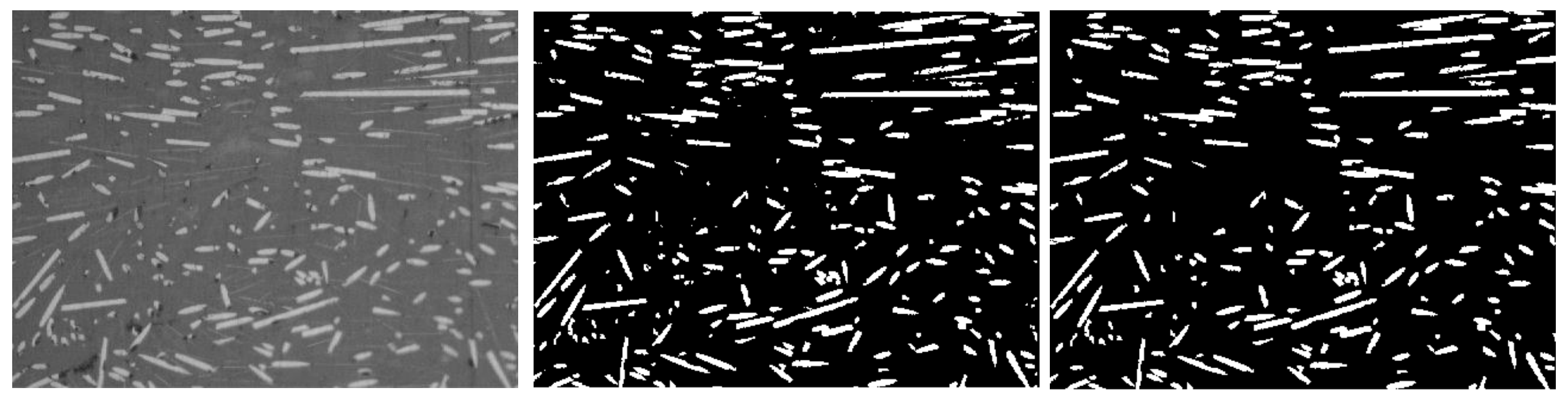

Figure 7-42 Fiber orientation computation process of a microscope image (cross-section of $90^{\circ}$ direction, 20x)
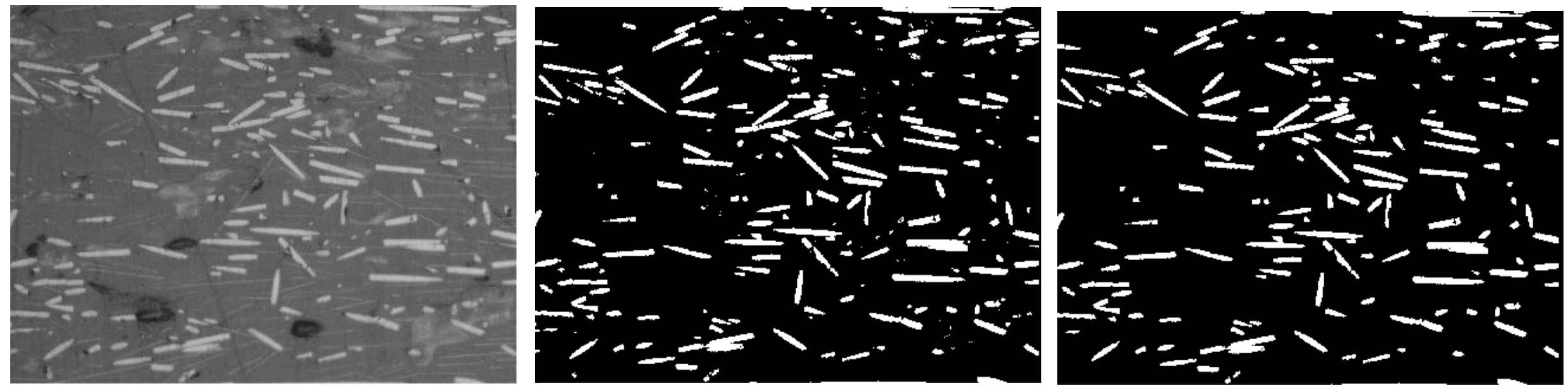

Figure 7-43 Fiber orientation computation process of a microscope image (cross-section of $90^{\circ}$ direction, 20x) 


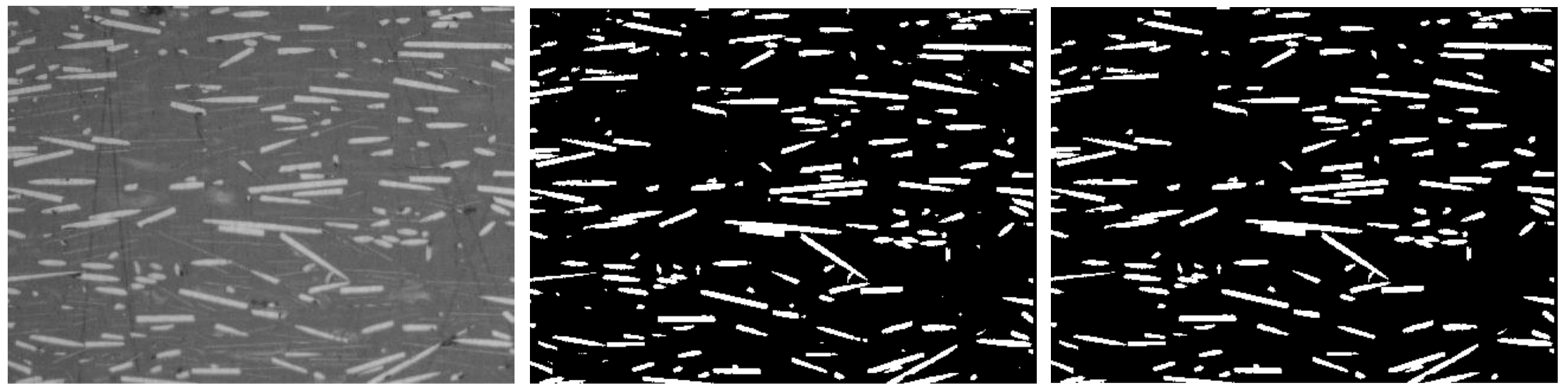

Figure 7-44 Fiber orientation computation process of a microscope image (cross-section of $90^{\circ}$ direction, 20x)
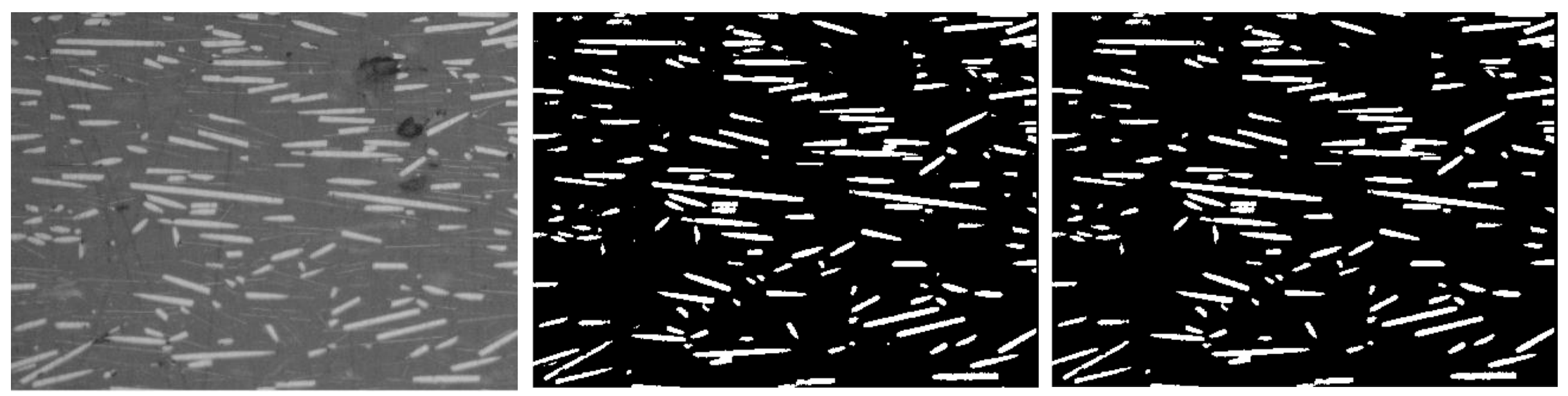

Figure 7-45 Fiber orientation computation process of a microscope image (cross-section of $90^{\circ}$ direction, 20x) 

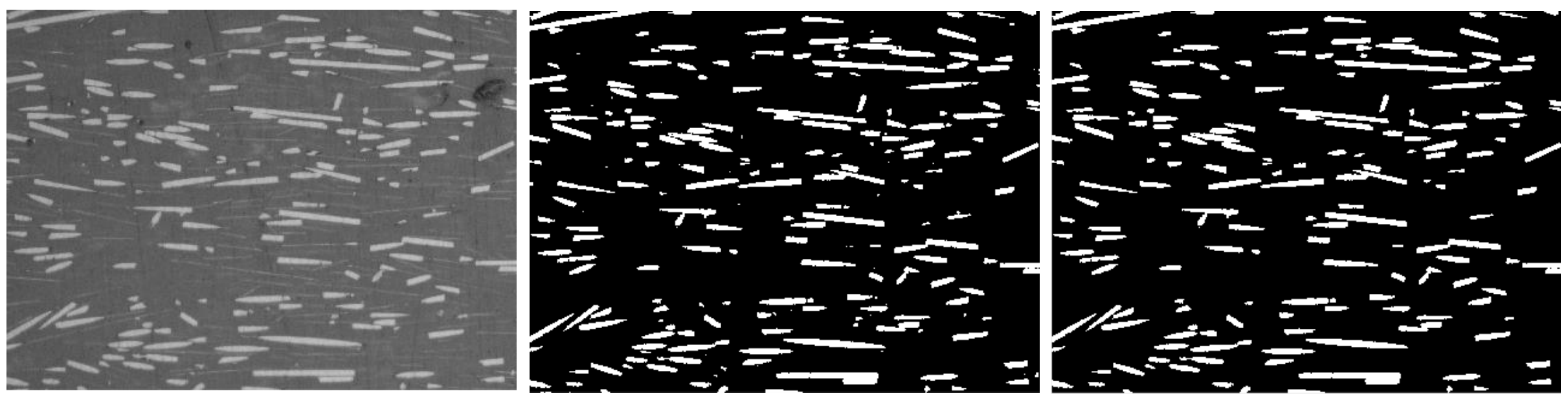

Figure 7-46 Fiber orientation computation process of a microscope image (cross-section of $90^{\circ}$ direction, 20x)
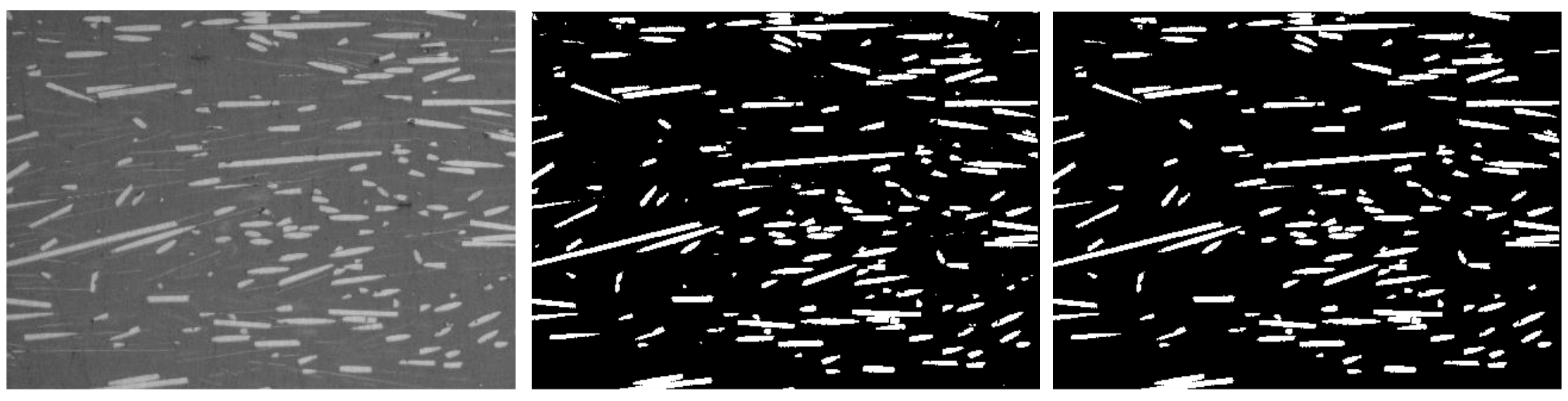

Figure 7-47 Fiber orientation computation process of a microscope image (cross-section of $90^{\circ}$ direction, 20x) 

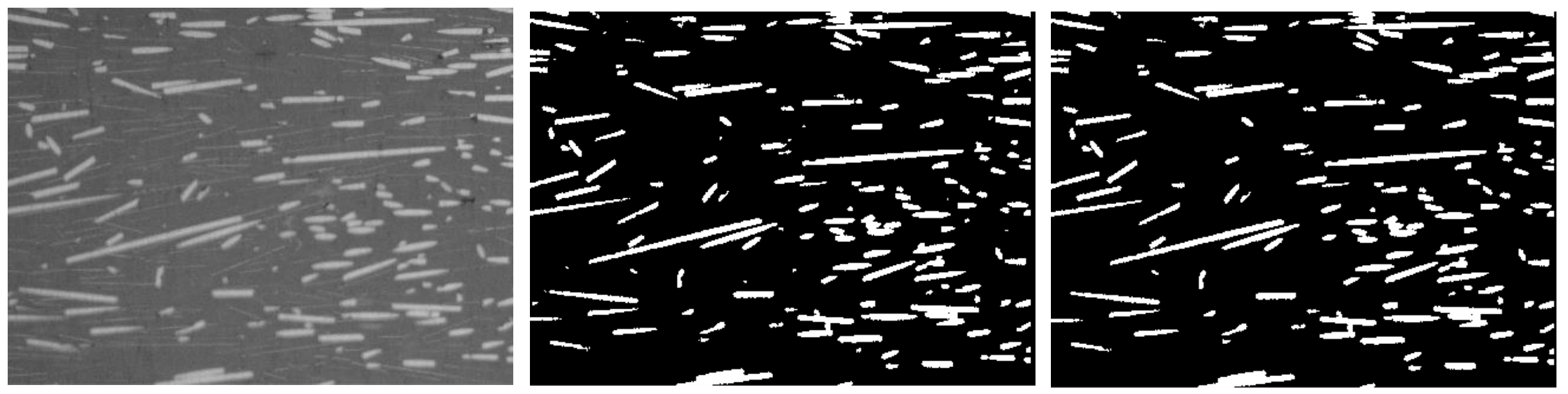

Figure 7-48 Fiber orientation computation process of a microscope image (cross-section of $90^{\circ}$ direction, 20x)
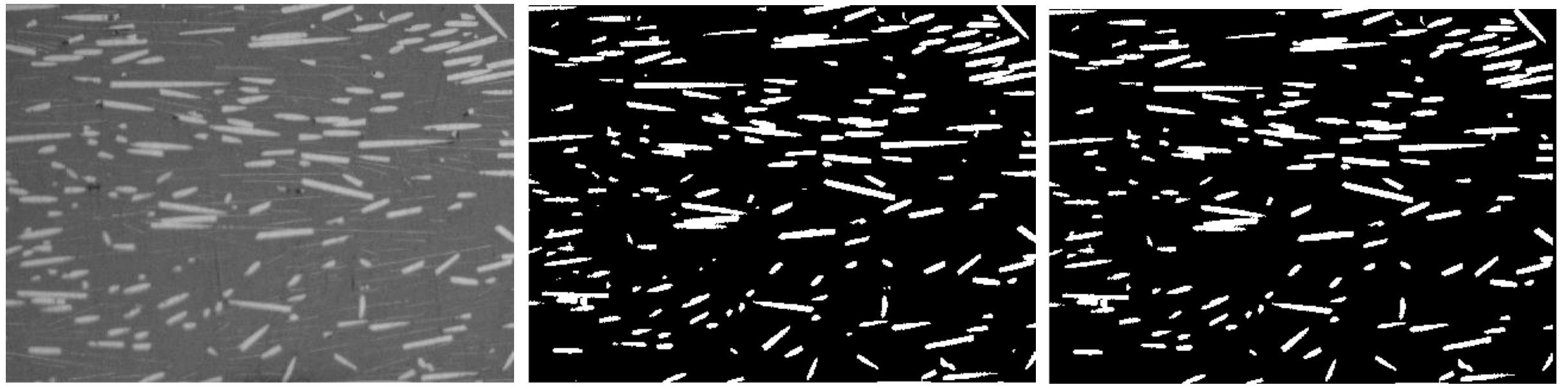

Figure 7-49 Fiber orientation computation process of a microscope image (cross-section of $90^{\circ}$ direction, 20x) 
H. Bending Test Results
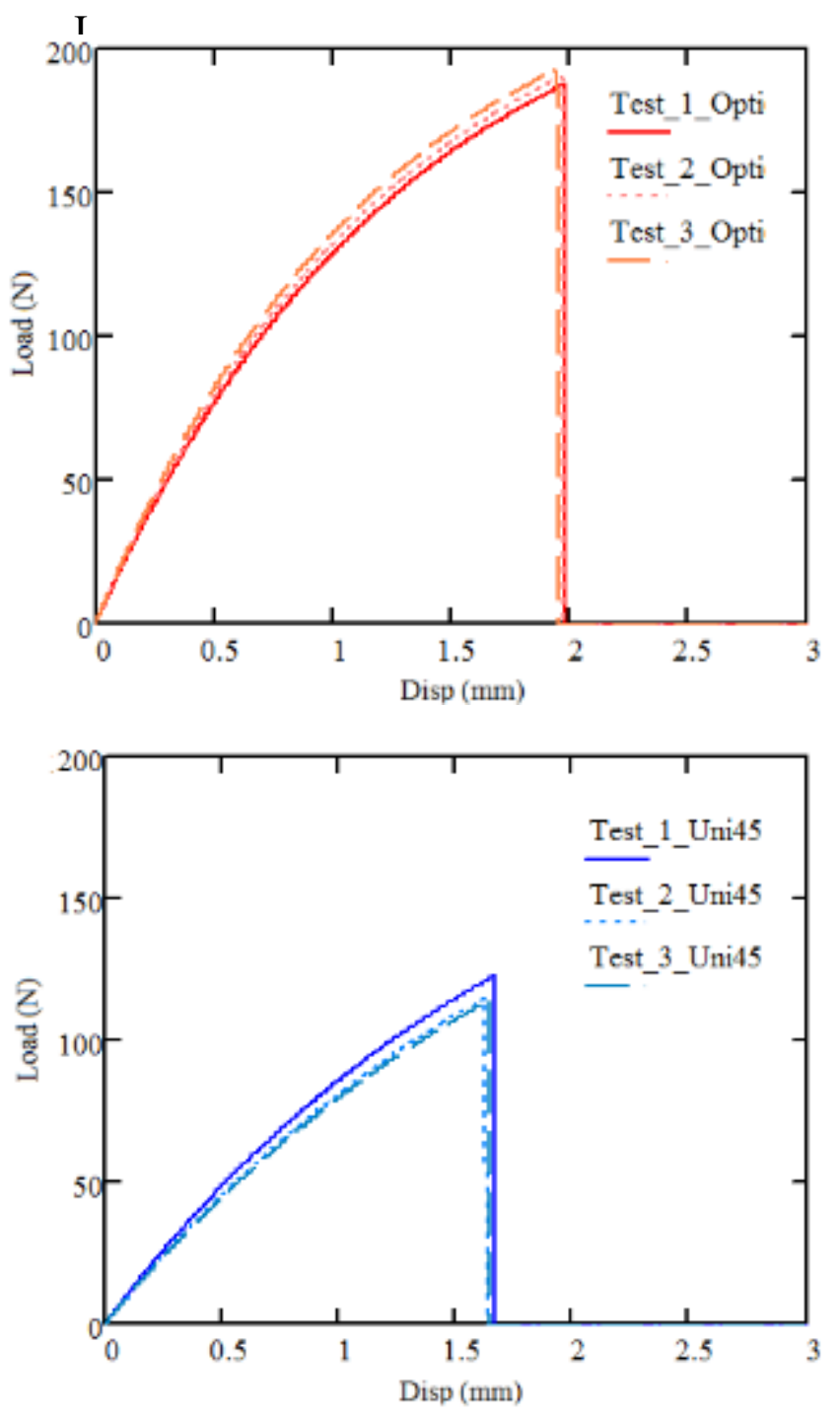
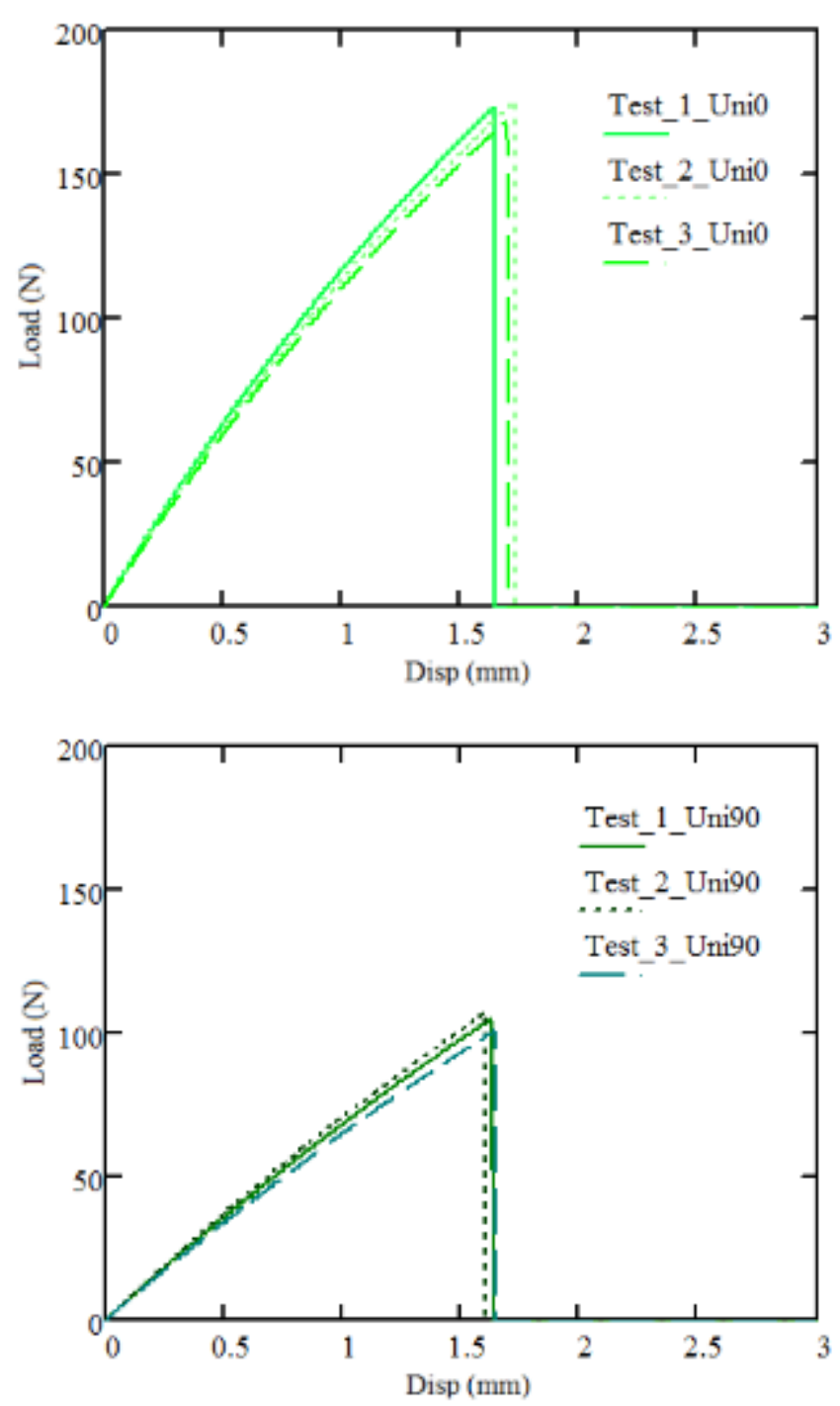

Figure 7-50 Three points bending test results of the beam printed at Optimized, $0^{\circ}, 45^{\circ}$, and $90^{\circ}$, respectively 
I. Gear test results

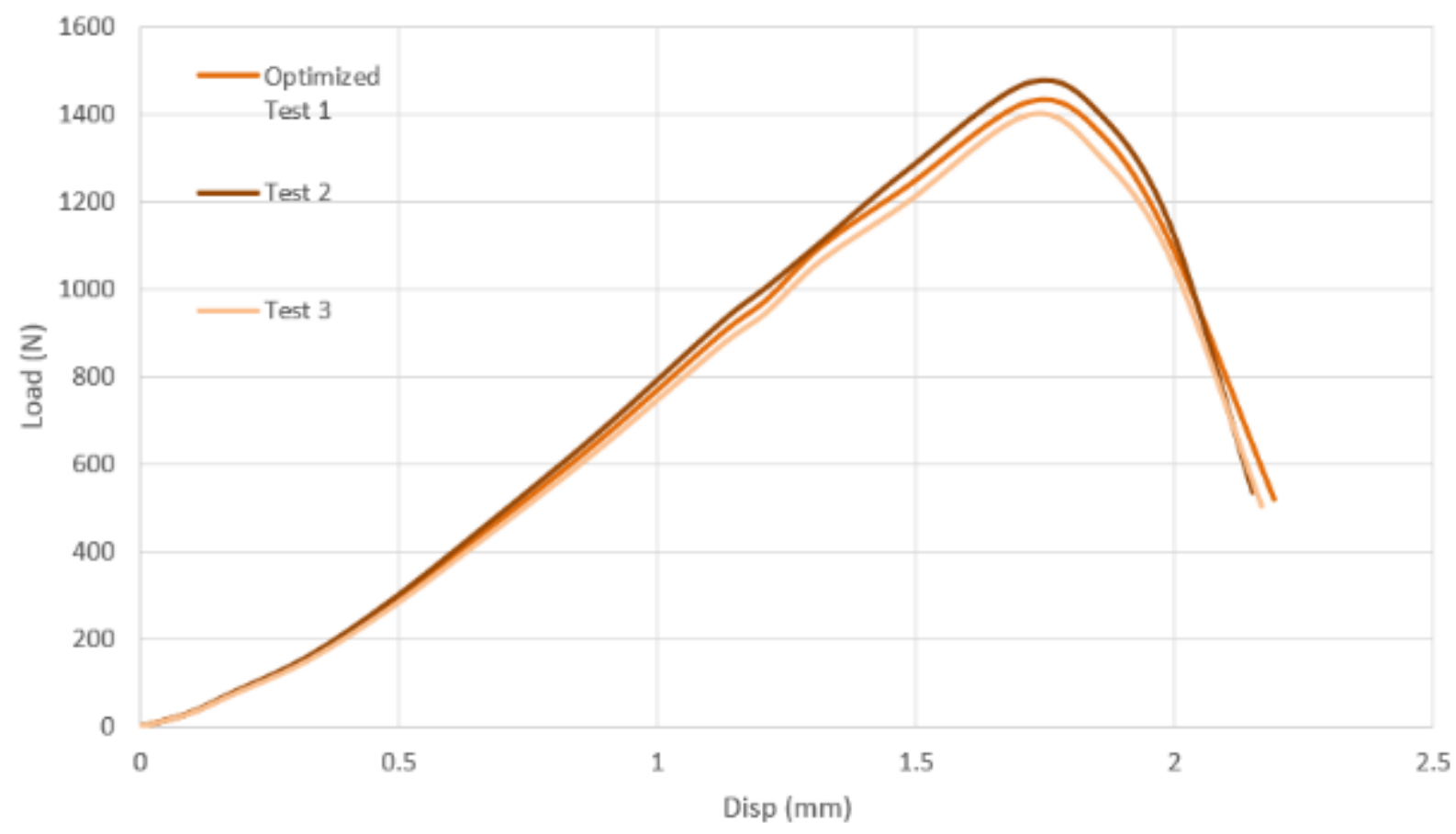

Figure 7-51 Load vs displacement for the gear printed at the optimized tool-path

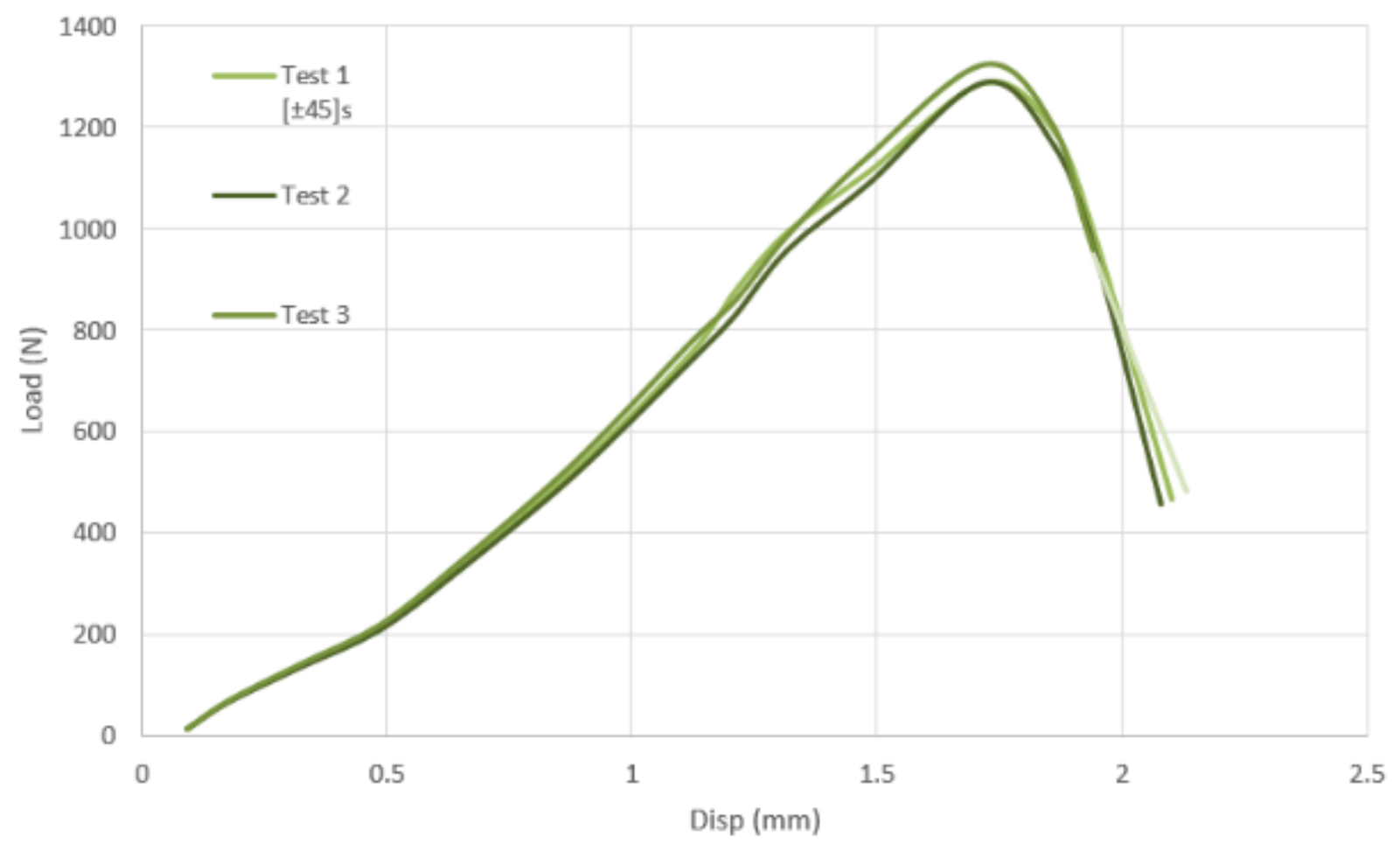

Figure 7-52 Load vs displacement for the CFRP gear printed at $\pm 45^{\circ}$ 


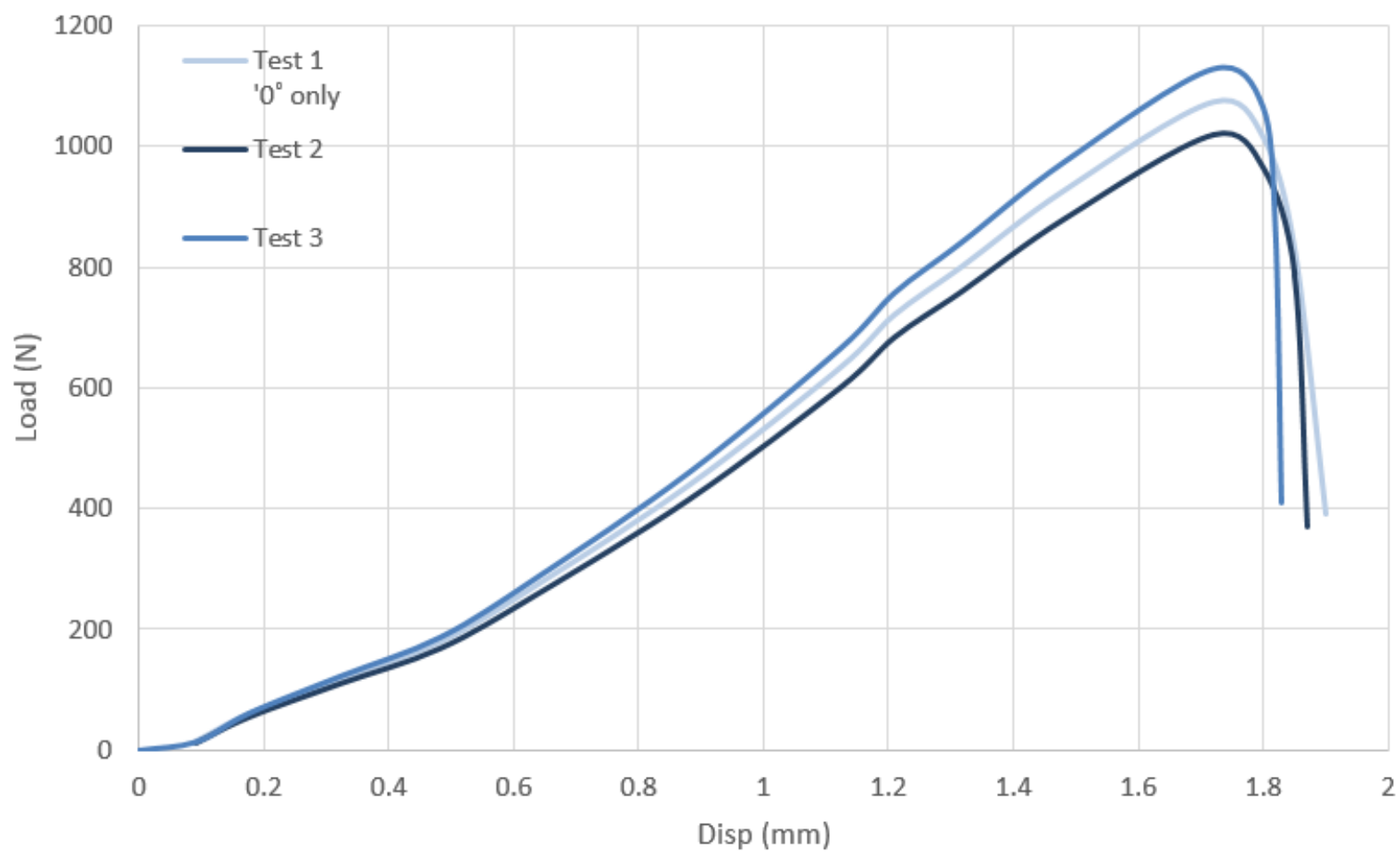

Figure 7-53 Load vs displacement for the CFRP gear printed at $0^{\circ}$ only

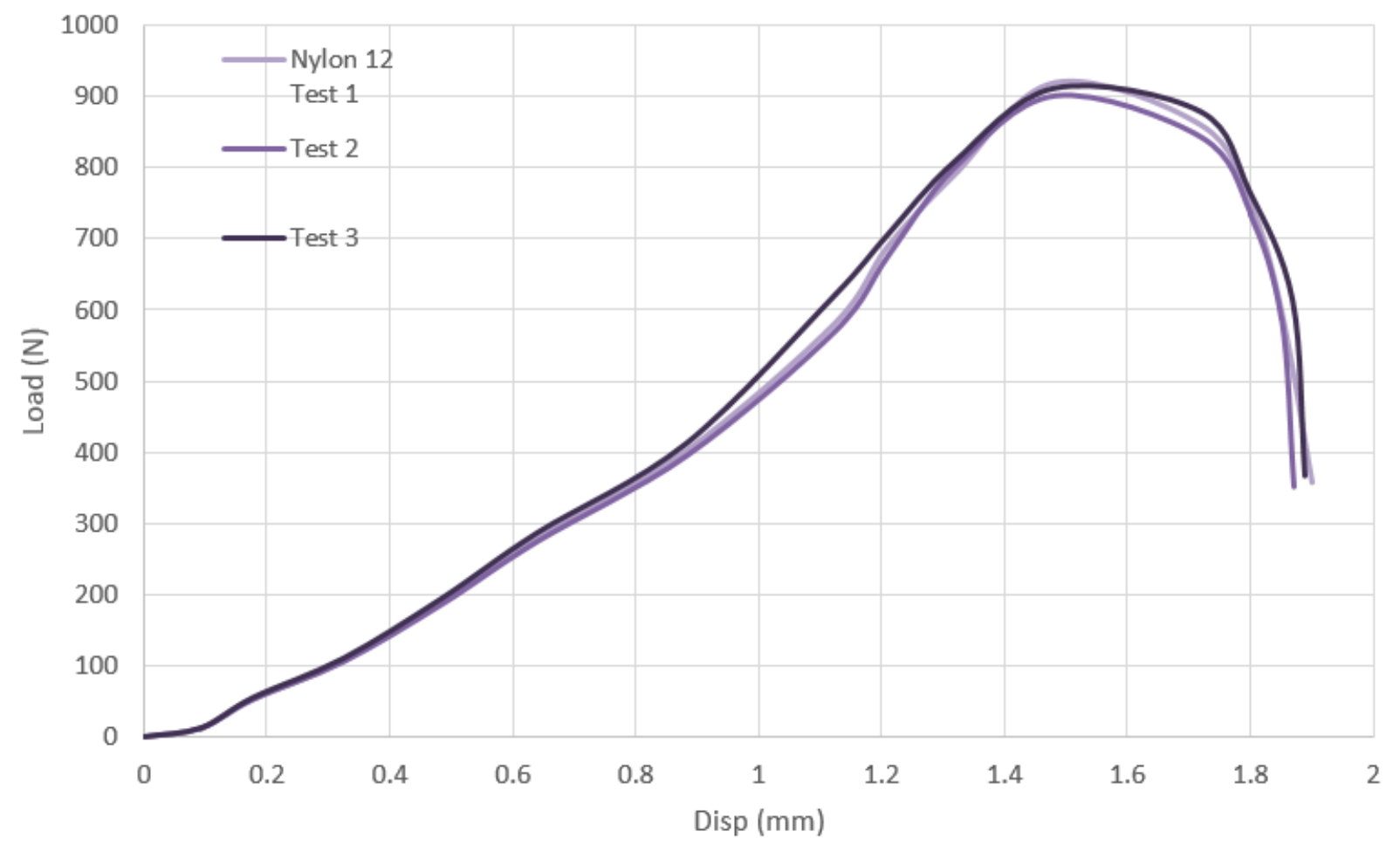

Figure 7-54 Load vs displacement for the Nylon 12 gear printed at optimized tool-path 


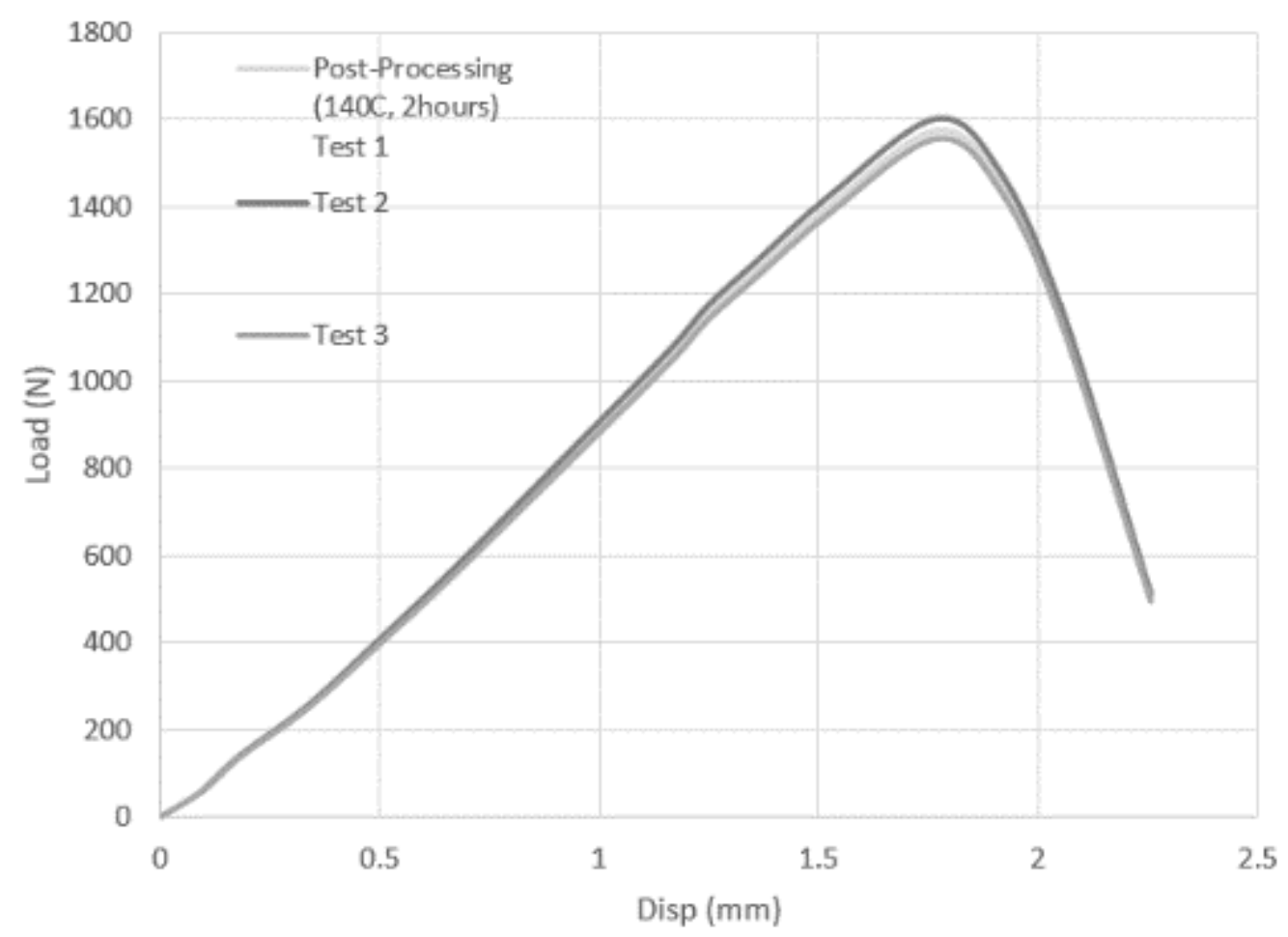

Figure 7-55 Load vs displacement for the CFRP gear after annealing under $140{ }^{\circ} \mathrm{C}$

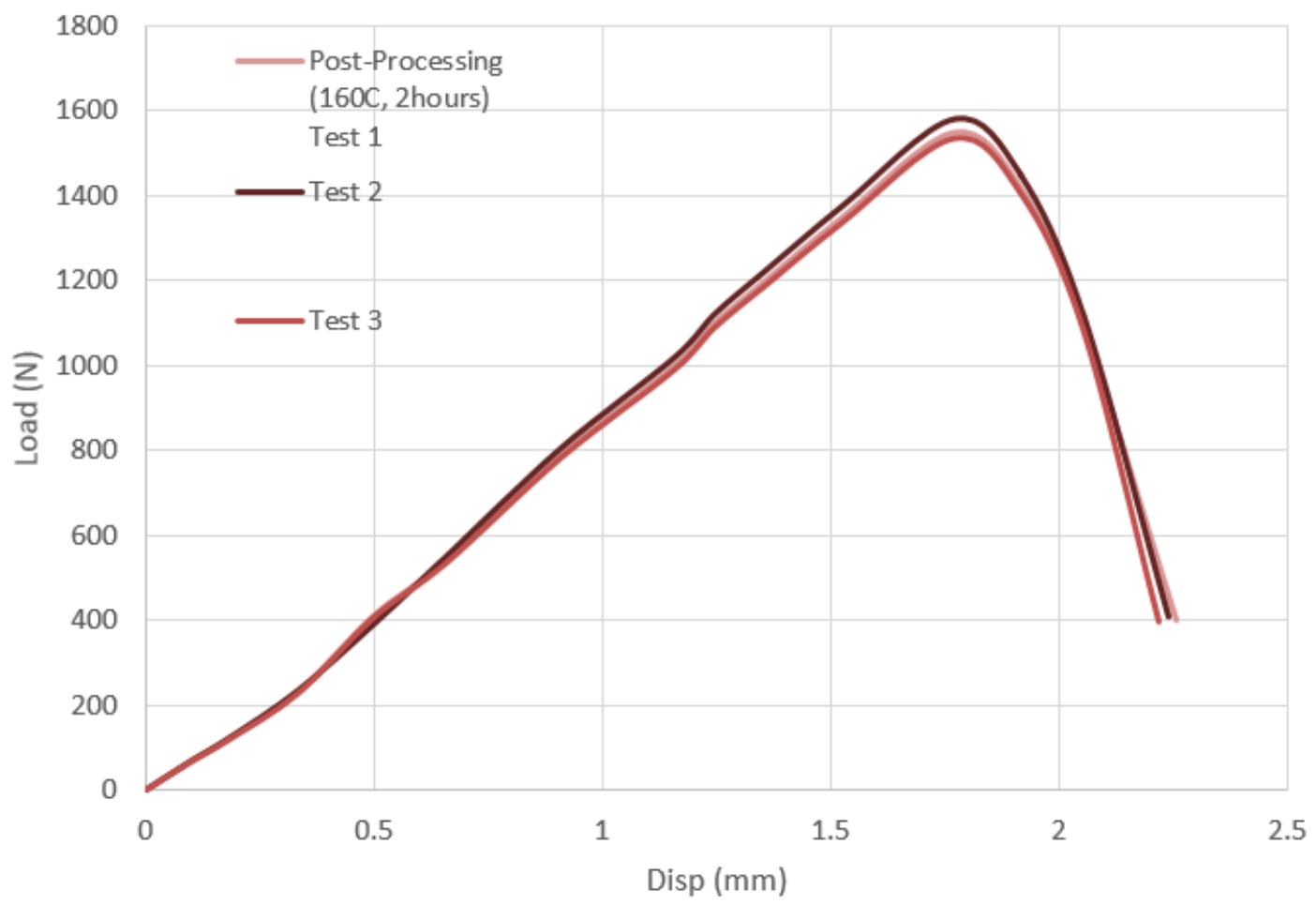

Figure 7-56 7-57 Load vs displacement for the CFRP gear after annealing under $140^{\circ} \mathrm{C}$ 
J. Strain stress curves of samples printed various nozzle diameters

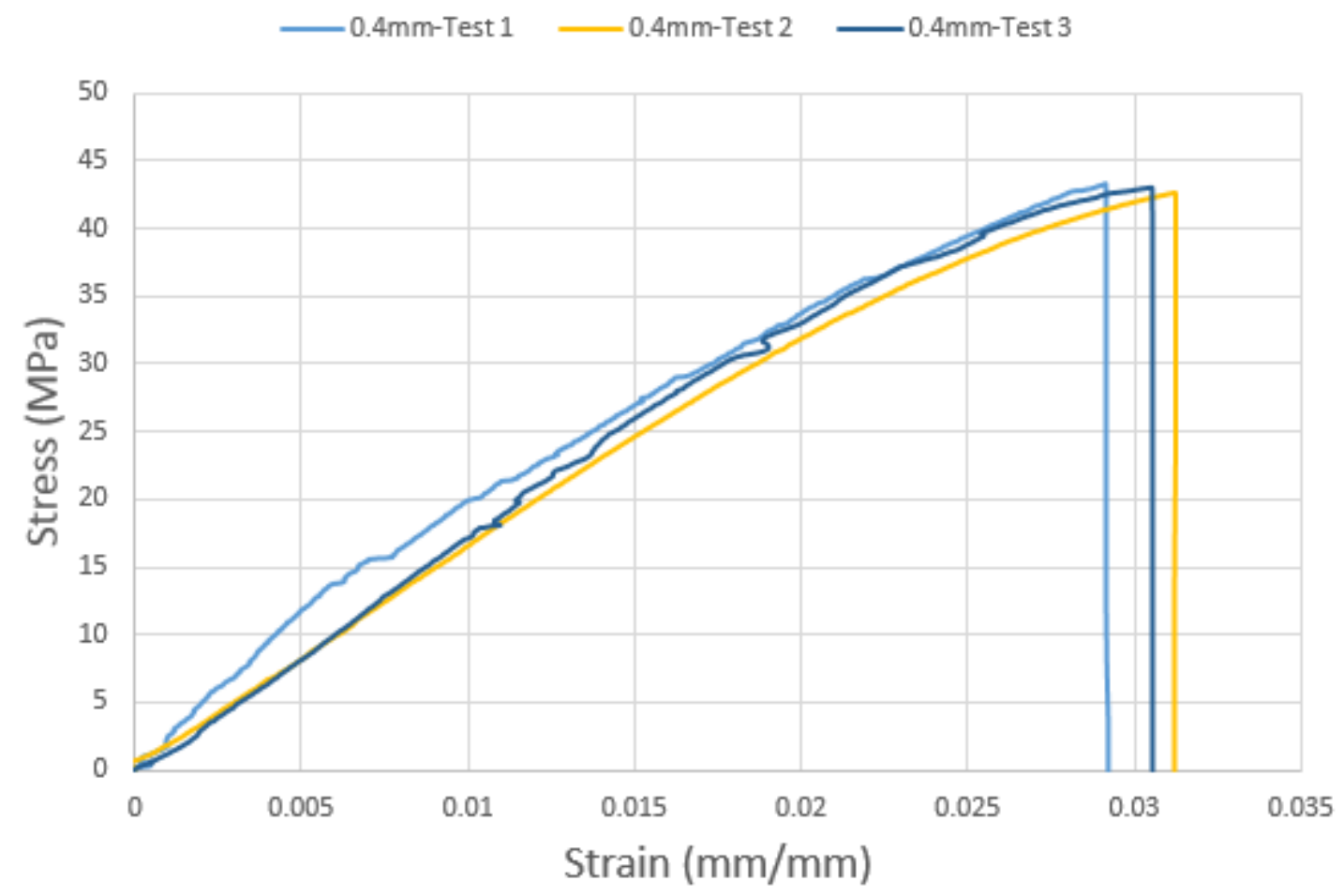

Figure 7-58 Tensile stress vs strain of samples printed by $0.4 \mathrm{~mm}$ nozzle

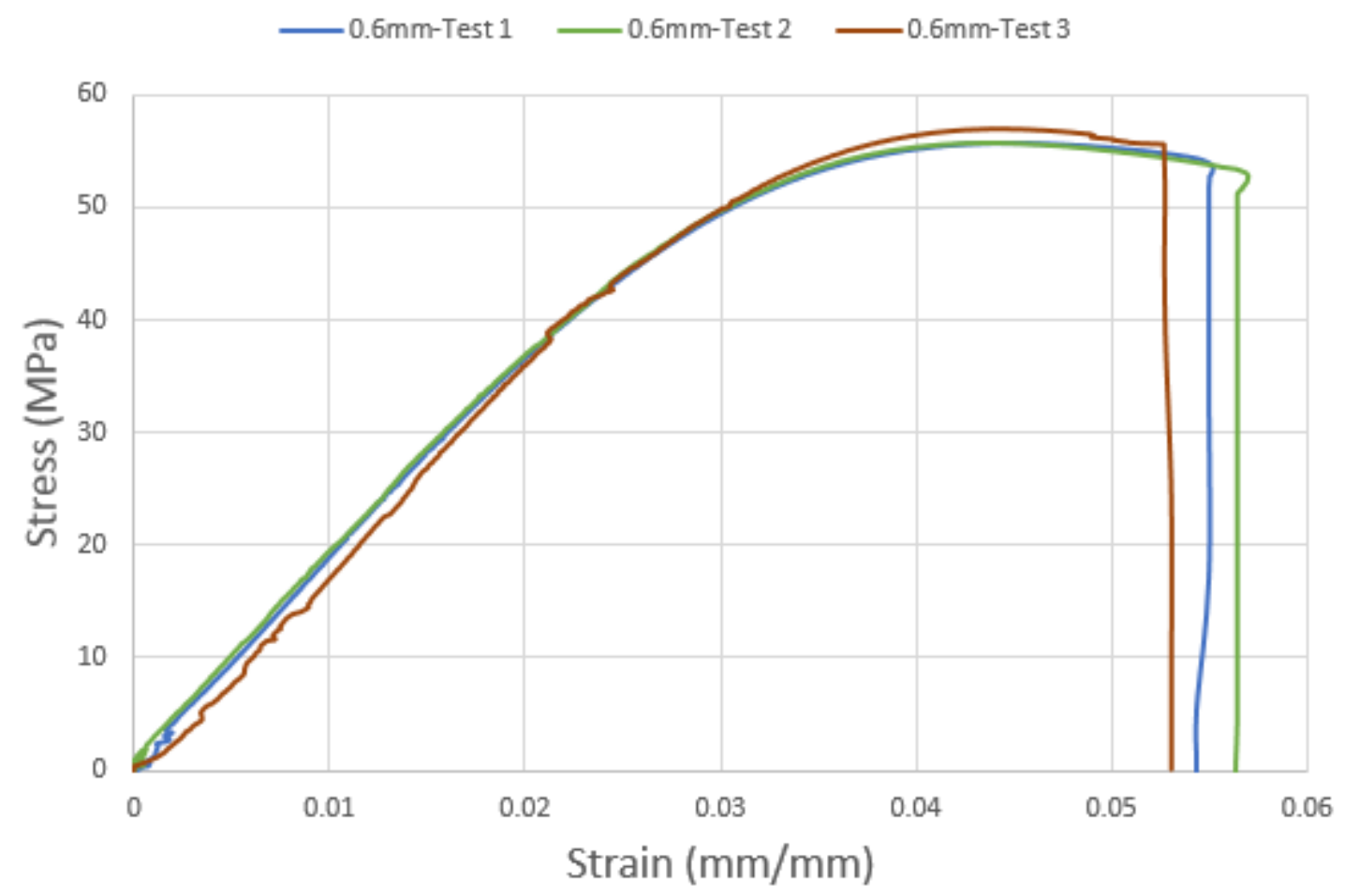

Figure 7-59 Tensile stress vs strain of samples printed by $0.6 \mathrm{~mm}$ nozzle 


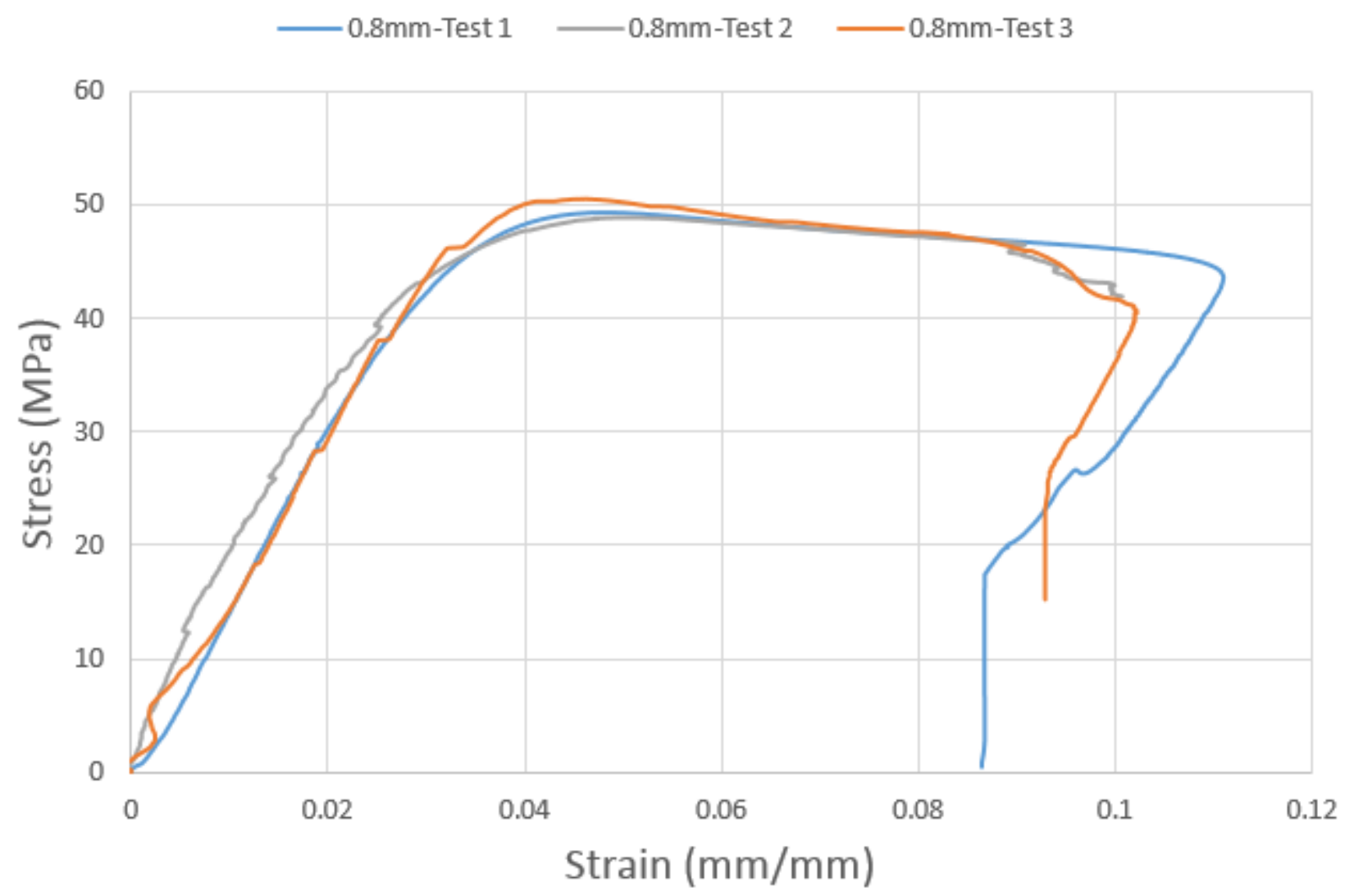

Figure 7-60 Tensile stress vs strain of samples printed by $0.8 \mathrm{~mm}$ nozzle 
K. Gear Force computation

\begin{tabular}{ccc}
\hline Definition & Equation & Value \\
\hline Horse power & $\mathrm{P}=0.75 \mathrm{kw}$ & $1.006 \mathrm{hp}$ \\
Torque & $T=\frac{60000 * P}{r p m}$ & $18 \mathrm{kN} * \mathrm{~mm}$ \\
Transmitted force & $F_{t}=\frac{2 * T}{d}$ & $494.3 \mathrm{~N}$ \\
Resultant Force & $F_{r}=\frac{F_{t}}{\operatorname{Cos}(\Theta)}$ & $526.0 \mathrm{~N}$ \\
\hline
\end{tabular}

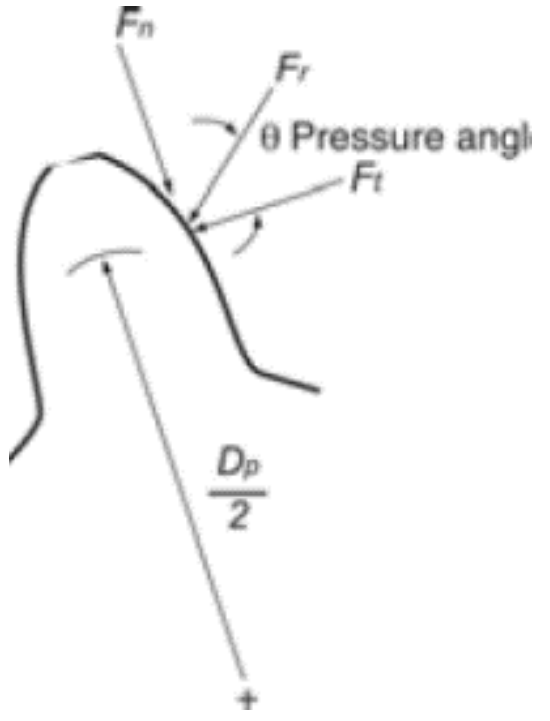

